\title{
The role of glutamate receptors in formation and maturation of Drosophila neuromuscular synapses
}

\author{
Dissertation \\ Zur Erlangung des Doktorgrades \\ der Mathematisch-Naturwissenschaftlichen Fakultäten \\ der Georg-August-Universität zu Göttingen
}

Vorgelegt von

Andreas Schmid

aus München

Göttingen, 2006 
D7

Referent: Prof. Dr. Ernst A. Wimmer

Korreferent: Prof. Dr. Ralf Heinrich

Tag der mündlichen Prüfung: 02.11.2006 


\section{The Fly - Ogden Nash (1902-1971)}

God in his wisdom made the fly

And then forgot to tell us why.

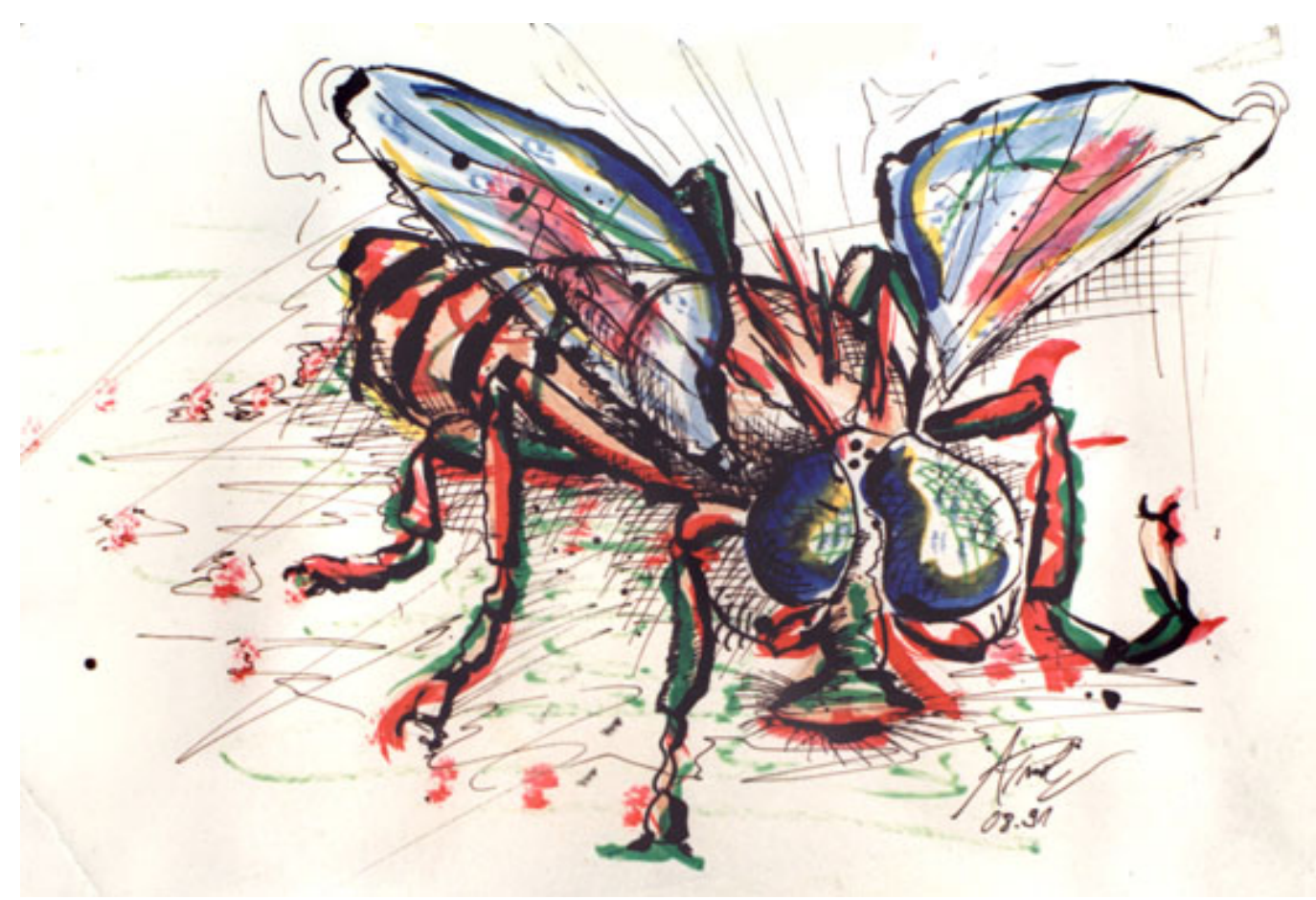




\section{$\underline{\text { Table of contents }}$}

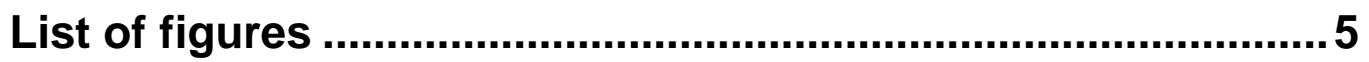

List of tables .................................................................................. 7

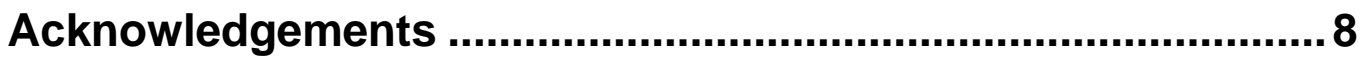

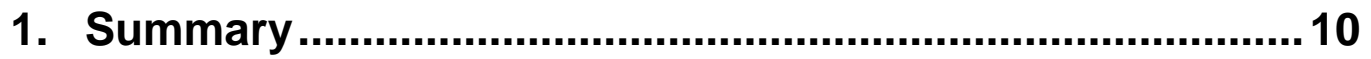

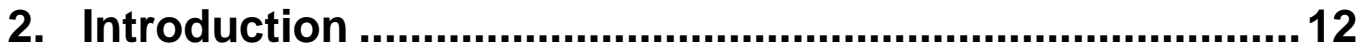

2.1. Structure and function of synapses .............................................12

2.1.1. Vertebrate neuromuscular synapses........................................ 13

2.1.2. Excitatory vertebrate CNS synapses.......................................... 14

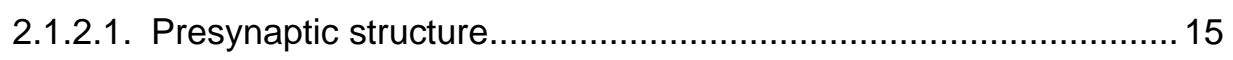

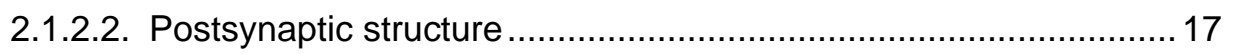

2.1.2.3. Structural synaptic plasticity and LTP .................................... 18

2.1.3. Drosophila neuromuscular synapses ............................................ 19

2.1.3.1. Drosophila melanogaster as a model system ............................. 19

2.1.3.2. Properties of the Drosophila NMJ .......................................... 20

2.1.3.3. Development of the Drosophila NMJ....................................... 22

2.1.3.4. Glutamatergic NMJ synapses ............................................... 23

2.1.3.5. Activity-dependent remodeling ............................................. 24

2.2. Structure and function of ionotropic glutamate receptors ..........25

2.2.1. Structure and assembly of non-NMDA glutamate receptors ................ 25

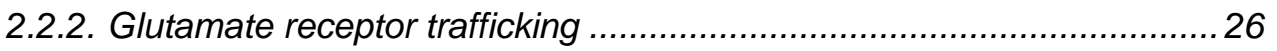

2.2.3. Glutamate receptors at Drosophila NMJ synapses ...........................28

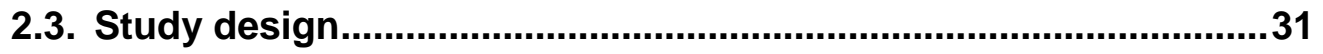

3. Material and Methods......................................................... 32

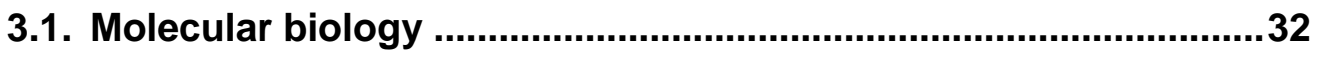

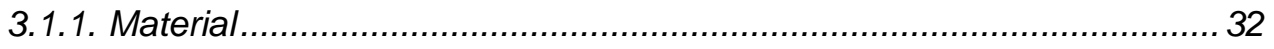

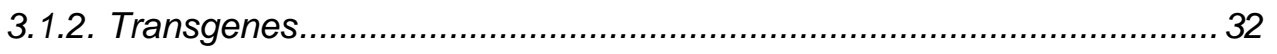

3.1.2.1. Site-directed fluorescence-tagging of gluRIIA and gluRIIB ..........34

3.1.2.2. Chimeric glutamate receptor subunits....................................... 36

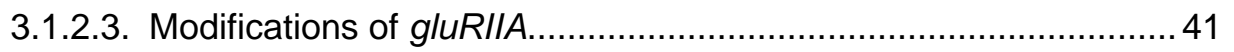

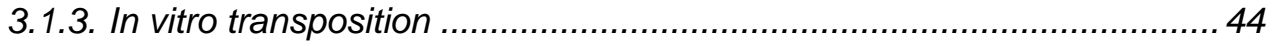

3.2. Drosophila melanogaster ..........................................................45

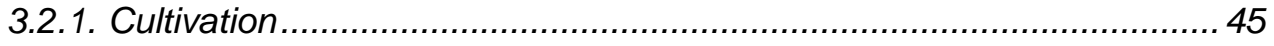

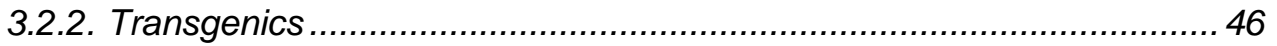

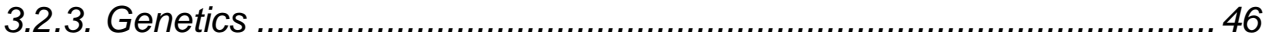




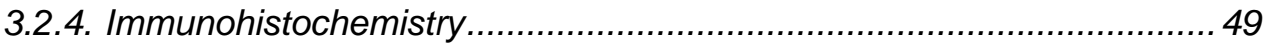

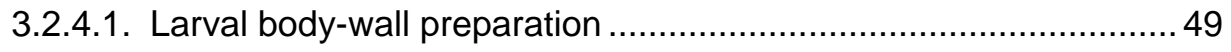

3.2.4.2. Embryonic body-wall preparation ......................................... 49

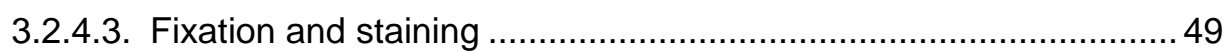

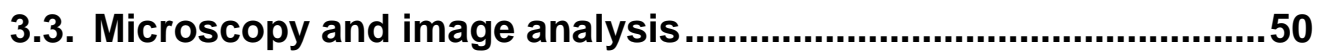

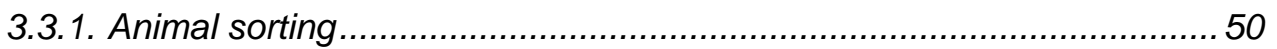

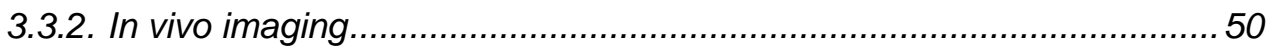

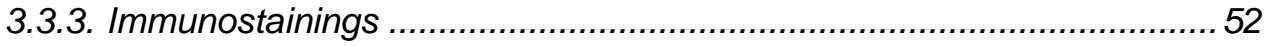

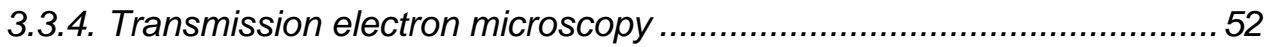

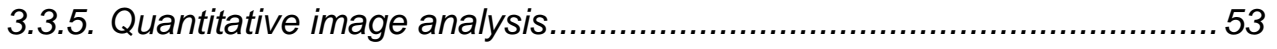

3.3.5.1. In vivo imaging - basic procedure ........................................... 53

3.3.5.2. In vivo imaging - Tracing and development of single PSDs .........55

3.3.5.3. In vivo imaging - FRAP experiments .......................................55

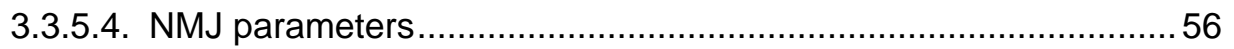

3.3.5.5. Comparison of absolute signal intensities ................................. 57

3.3.5.6. Determination of intensity profiles ........................................... 57

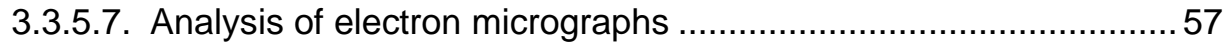

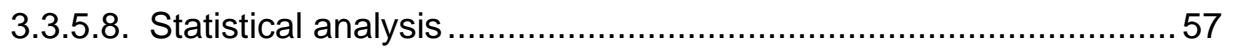

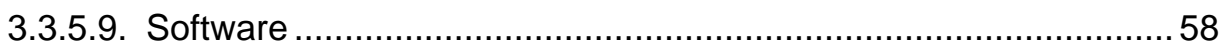

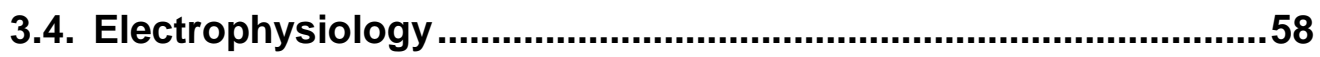

3.4.1. Patch clamp recordings ............................................................ 58

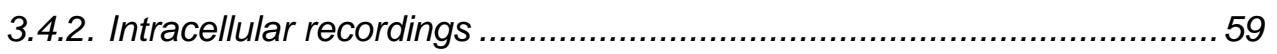

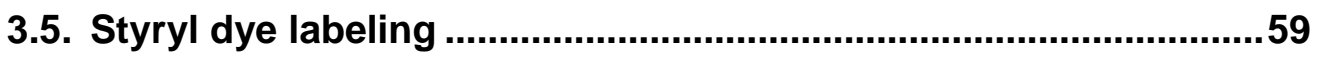

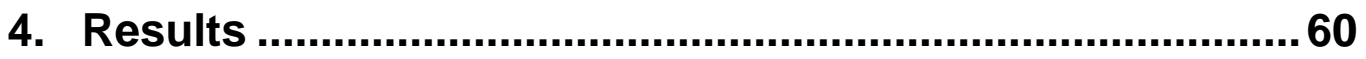

4.1. Non-NMDA type glutamate receptors are essential for maturation but not for initial assembly of synapses at Drosophila NMJs .............60

4.1.1. Formation of glutamatergic NMJ synapses deprived of postsynaptic

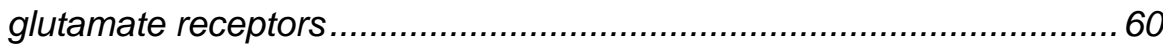

4.1.2. Presynaptic release sites are functionally and structurally maintained at glutamate receptor deprived NMJ synapses ...................................... 62

4.1.3. Early stop of postsynaptic maturation at glutamate receptor deprived

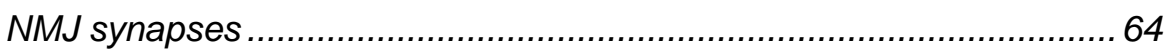

4.1.4. Ultrastructural analysis: lack of apposition between pre- and postsynaptic membranes at NMJ synapses deprived of glutamate receptors. 66

4.1.5. Initial molecular assembly of PSDs independent of glutamate receptors.. 
4.1.6. Neurotransmission and glutamate-triggered ionic conductance are dispensable for NMJ synapse maturation and growth.....

4.1.7. C-terminal truncation of GluRIIA mimics the receptor deprivation defects 72

4.1.8. Postsynaptic assembly seems to require intracellular sequence elements common to GluRIIA, IIB and IIC. . .73

4.2. Fluorophore-tagging of glutamate receptor subunits .................76

4.2.1. Random insertion - In vitro transposition ...................................... 77

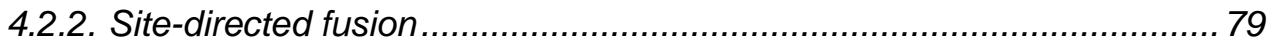

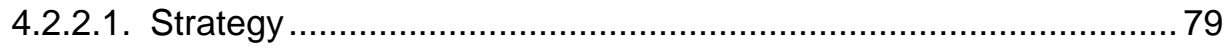

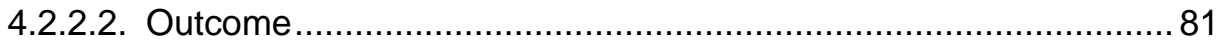

4.3. Subunit-specific targeting of glutamate receptors organizes PSD

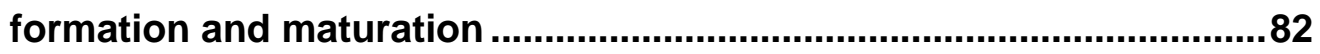

4.3.1. Functional GFP fusions of GluRIIA and IIB to in vivo study synapse formation at developing NMJs of Drosophila..................................... 82

4.3.2. Simultaneous in vivo imaging of GluRIIA and GluRIIB ....................... 85

4.3.3. Receptor composition balances in the course of PSD growth and maturation... 87

4.3.4. Distinct PSD incorporation behavior of GluRIIA and GluRIIB. 89

4.3.5. The CTD of GluRIIA contributes to suppress GluRIIA incorporation at mature PSDs

4.3.6. Distinct PSD incorporation of GluRIIA and IIB persists in the absence of the other complex.

4.3.7. GluRIIA is needed to allow efficient growth of PSDs and to define mature PSD size..... .94

4.3.8. GluRIIA dominates synaptic transmission . 96

4.3.9. GluRIIA PSD content is selectively enhanced after blockade of presynaptic glutamate release.

4.3.10. Receptor content per PSD can be down-regulated to allow the formation of additional synapses during experience-dependent plasticity .......... 100

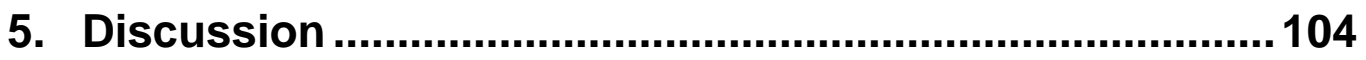

5.1. Non-NMDA type glutamate receptors are essential for maturation but not for initial assembly of synapses at Drosophila NMJs........... 104

5.1.1. A transmission independent role of glutamate receptors in postsynaptic maturation. 104

5.1.2. Ultrastructural and molecular maturation of NMJ synapses requires glutamate receptors 104 
5.1.3. Glutamate receptor complexes and synaptic cell adhesion 106

5.1.4. Glutamate receptor levels in control of synapse formation 107

5.2. Functional fluorophore-tagging of glutamate receptor subunits.... 109

5.3. Subunit-specific targeting of glutamate receptors organizes PSD formation and maturation

5.3.1. Subunit-specific glutamate receptor trafficking

5.3.2. Factors controlling the differential trafficking of GluRIIA and GluRIIB 112

5.3.3. Phosphorylation and receptor trafficking ...

5.3.4. Activity-dependent PSD regulation - blocked neurotransmission ....... 115

5.3.5. Activity-dependent PSD regulation - enhanced neurotrans-mission .. 116

5.3.6. Physiological relevance of GluRIIA and GluRIIB complexes. 118

6. References ............................................................... 121

6.1. Scientific articles and monographies .........................................121

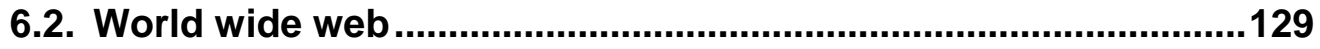

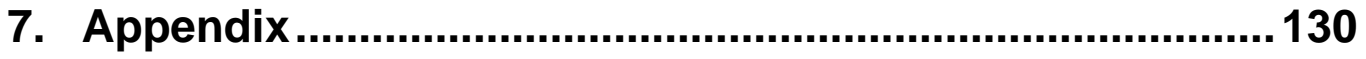

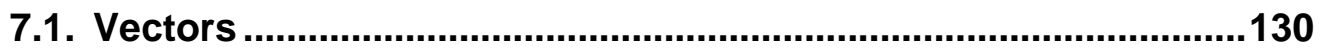

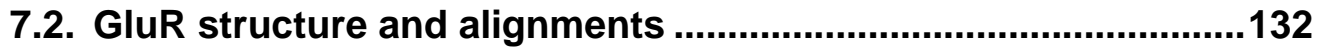

7.3. Constructs and transgenes ........................................................135

7.4. In vivo imaging device..................................................................138

7.5. Normalization of the PSD number and NMJ size....................... 138

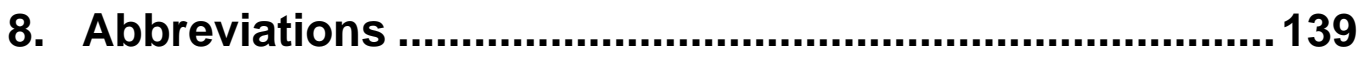

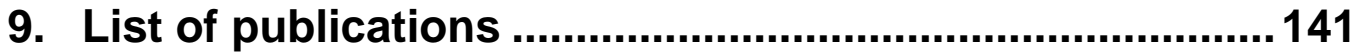

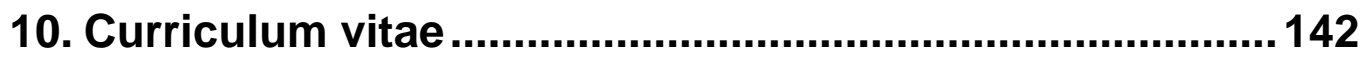




\section{List of figures}

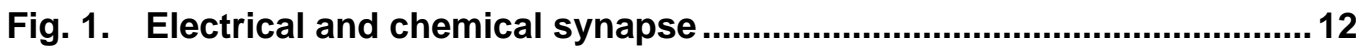

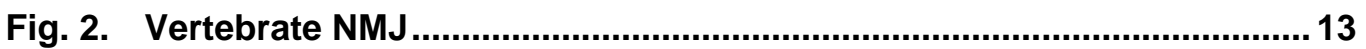

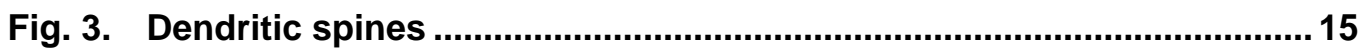

Fig. 4. Model of dendritic spine development..................................................... 15

Fig. 5. Molecular components of the CAZ and the active zone........................16

Fig. 6. Molecular components of the postsynaptic density (PSD).................. 17

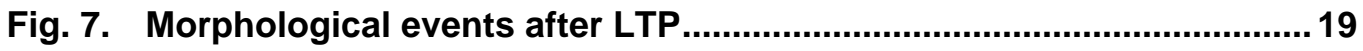

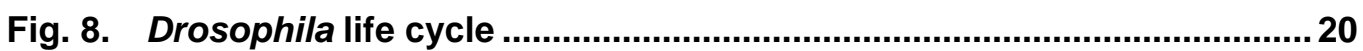

Fig. 9. Drosophila larval body wall muscles ................................................. 21

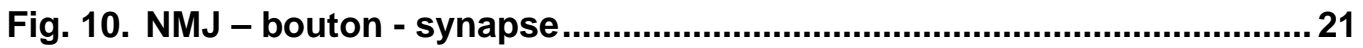

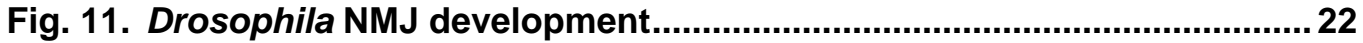

Fig. 12. Ultrastructure of glutamatergic Drosophila NMJ synapses................... 24

Fig. 13. Assembly of non-NMDA glutamate receptors ..................................... 26

Fig. 14. Subunit-dependent AMPA receptor insertion and recycling ................. 28

Fig. 15. Receptor subunit composition regulates desensitization kinetics...... 29

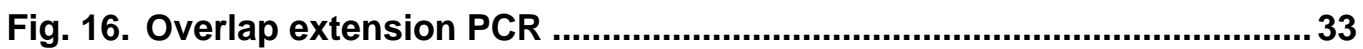

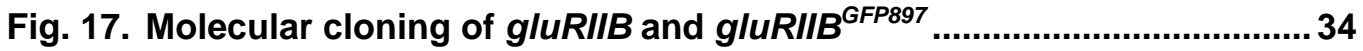

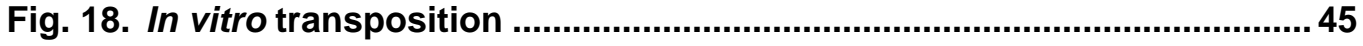

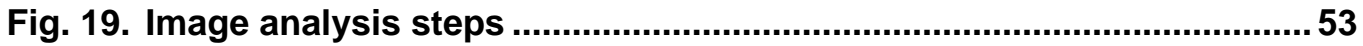

Fig. 20. Larval NMJs developing with extremely reduced levels of postsynaptic glutamate receptors..............................................................................61

Fig. 21. Active zones and presynaptic release at glutamate receptor deprived NMJ synapses

Fig. 22. Signs of chronically increased release probability at glutamate

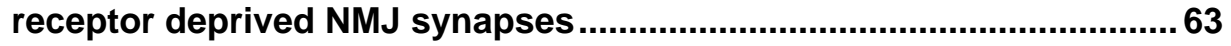

Fig. 23. Molecular markers of postsynaptic assembly at glutamate receptor deprived NMJ synapses

Fig. 24. Electron microscopic analysis of glutamate receptor deprived NMJ synapses 67

Fig. 25. Synapse assembly at embryonic NMJs lacking all glutamate receptors

Fig. 26. NMJ synapse assembly after suppression of neurotransmission or glutamate receptor ligand binding ........................................................ 70

Fig. 27. PSD assembly defects after C-terminal truncation of GluRIIA .............73

Fig. 28. GluR AA alignment: C-terminal domain (CTD) .................................. 74

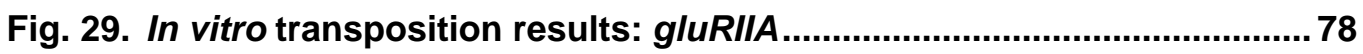


Fig. 30. In vitro transposition results: gluRIIB ............................................... 78

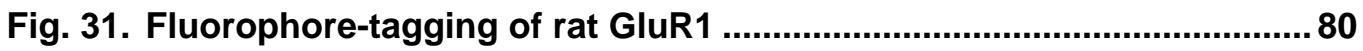

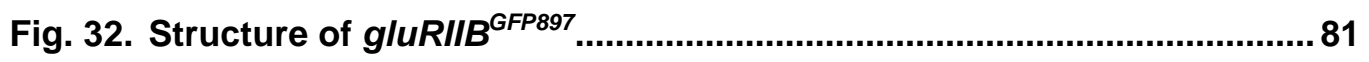

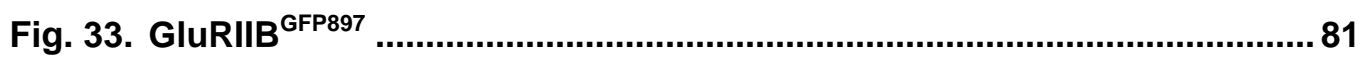

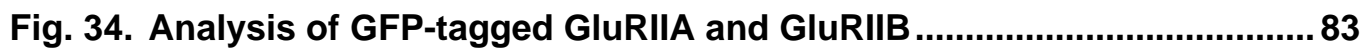

Fig. 35. Size-dependent divergence of PSD subunit composition. .................... 86

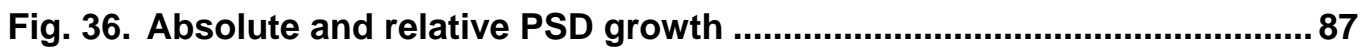

Fig. 37. PSD subunit composition development depends on initial stage ........ 88

Fig. 38. Differential PSD incorporation behavior of GluRIIA and GluRIIB

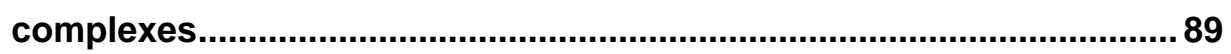

Fig. 39. Quantification of the differential FRAP behavior ................................. 91

Fig. 40. The differential incorporation characteristics involve the receptor $C$ -

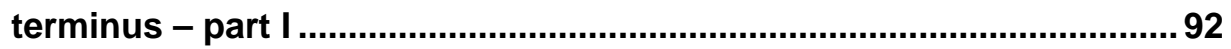

Fig. 41. Specific incorporation behavior independent of the presence of the respective $2^{\text {nd }}$ receptor type................................................................93

Fig. 42. The differential incorporation characteristics involve the receptor $\mathrm{C}$ terminus - part II .............................................................................. 94

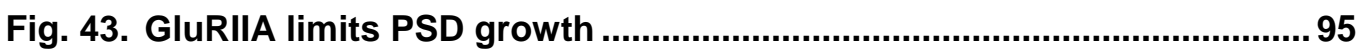

Fig. 44. Physiological characterization of GluRIIA and GluRIIB complexes..... 96

Fig. 45. Expression pattern of ok319-gal4......................................................... 97

Fig. 46. PSD composition after blockage of presynaptic release .......................98

Fig. 47. FRAP in the absence of the $2^{\text {nd }}$ receptor type after presynaptic activity

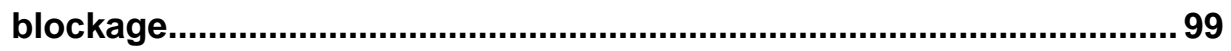

Fig. 48. GluRIIA and GluRIIB after activity blockage........................................ 100

Fig. 49. PSDs during experience-dependent plasticity................................... 101

Fig. 50. Activity and long-term NMJ plasticity ............................................. 102

Fig. 51. Model: Maturation of PSDs requires glutamate receptors .................. 105

Fig. 52. Model: Transsynaptic interactions instructed by intracellular

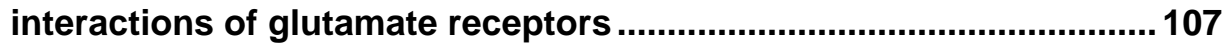

Fig. 53. Model: Differential glutamate receptor incorporation involves the

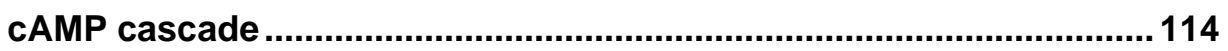

Fig. 54. Model: Presynaptic activity, postsynaptic conductance and PSD

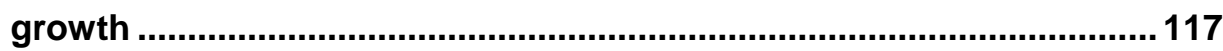

Fig. 55. Model: PSD growth in the sole presence of either GluRIIA or GluRIIB...

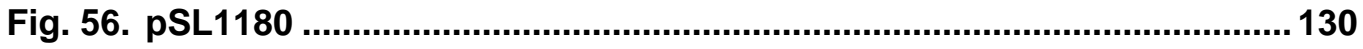

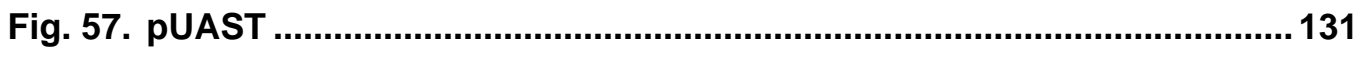


Fig. 58. Crystal structure of glutamate bound GluR6 - S1/S2 domain............. 132

Fig. 59. GluR AA alignment: S1/S2 domain - tetramer stabilization................. 132

Fig. 60. GluR AA alignment: S1/S2 domain - beta 7,8, helix D and J ............. 133

Fig. 61. GluR AA alignment: reentrant pore loop and M2 .............................. 134

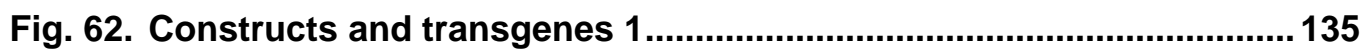

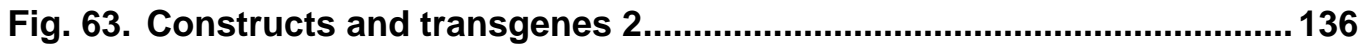

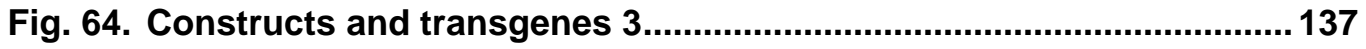

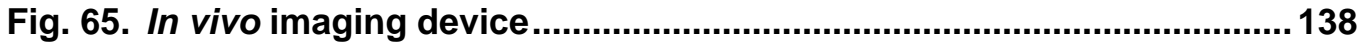

\section{List of tables}

Table 1. Fly stocks for germ line transformation .................................................46

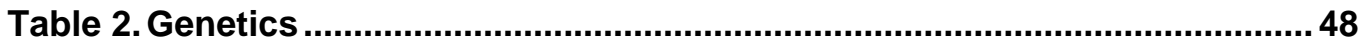

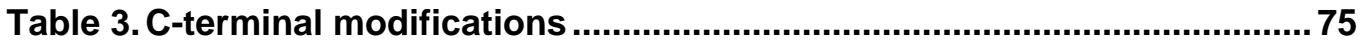




\section{Acknowledgements}

Foremost, I would like to thank my supervisor Prof. Stephan Sigrist for his continuous support and advice, for fruitful scientific work and discussions and the consistent full confidence he had in me. Furthermore, I am also grateful to Prof. Ernst Wimmer and Prof. Ralf Heinrich for their valuable support as my thesis committee and to Prof. Jörg Stülke, Prof. Michael Hörner, PD Michael Hoppert and Prof. Dieter Heineke for accepting the office as further members of my examination board.

I would like to thank all the members of the ENI and especially everybody from the AG Sigrist for creating a pleasant and amicable atmosphere.

In particular thanks go to:

- my dear friend Christine Quentin for being an excellent lab manager, my lab jester, a fantastic cook, kind, straightforward and considerate

- my dear friend Philipp Gorlovoy for his reliability, solidarity and hilarity

- Carolin Wichmann for doing electron microscopy, for her numerous perusals and for hard struggles whenever we had some treats in the lab

- Robert Kittel for almost all of the electrophysiological data in this thesis

- Andreas Frölich for the rest of the electrophysiological data in this thesis

- Qin Gang for starting up the IIA ${ }^{\text {hypo }}$ project

- Manuela Schmidt for being my bench buddy and fair-minded listener

- Tobias Schwarz for his helpfulness, enjoyable lunch breaks and being the best Beckenbauer double

- Wernher Fouquet and Sara Mertel for their cooperativeness

- Carola Sigrist, Rui Tian, Laura Swan, Tobias Rasse, Ulrike Prange, Carlos Merino, Thomas Schwarzbraun and Henrike Hartung for being likeable colleagues

- Jasmin Held, Miriam Richter, Franziska Zehe and Heiko Röhse for being technical aces (TAs)

- Anne Grünewald, my former diploma student, for her dedication on the IVT screen

- Andrea Möller and Banu Colak for being fly-mommies

- Magda Krause for her consistent support and smile

- Frank Kötting for being Daniel Düsentrieb

- Ulrike Borchardt for yummy fly food

- Oliver Schade and Matthias Weyl for support whenever I thought nothing else as a sledgehammer could help my PC 
- Wiebke Heinrich and Dagmar Thomiczek for their support with administrative concerns

Furthermore, I would like to thank Prof. Manfred Heckmann for his constructive advice.

Moreover, I would like to thank everybody who contributed to make my off work time in Göttingen enjoyable, diversified and prosperous: my flat-mates and neighbors, the Hochschulsport crew, the ASTA Sportreferat, the Monday evening soccer people, the Tuesday evening Spanish course, the Wednesday evening cast of the DT and especially my awesome Thursday evening Rollersoccer team, namely Tatjana and Tomi Haramina, Philipp Gorlovoy, Nicolas Bontems, Matthias Schmalisch, HansMatti Blencke, Fabian Commichau, Carola Eyßell, Justus Lücke, Franziska Jurk, Friederike Draber, Jörg Mertins, Dennis Rippe, Dennis Kappei, Steffen Arning, Johanna Kuhlenkampff, Monika Herold, Inga and Volker Hannstein.

Special thanks also goes to my dear friends Christian Graf and his wife Katja, Ulrich Syber and Markus Hermann, who accompanied me in an inimitable way throughout my studies.

Last but not least, I would like to thank my girlfriend Silke for her understanding, kindness and love and particularly my family and grandma for their lifelong support, dedication, frankness, guidance and faith in me.

Thanks to everybody else who accompanied me, believed in me and helped me finding the right path. 


\section{Summary}

Ionotropic glutamate receptor channels (GluRs) are key elements for excitatory neurotransmission in the central nervous system (CNS). Both the activity-dependent as well as the activity-independent trafficking of GluRs to postsynaptic densities (PSDs) of glutamatergic synapses is meant to control synaptic plasticity in the mammalian nervous system, thereby mediating learning and memory. Whether similar processes are generally implicated into the formation and adaptation of glutamatergic synapses, e.g. throughout nervous system development, remained unknown so far. The Drosophila neuromuscular junction (NMJ) is a highly accessible synaptic model system, which allows extensive in vivo analysis of cellular and molecular mechanisms by combining efficient genetics with physiological, ultrastructural and histological analyses. Two glutamate receptor complexes, containing either the subunit GluRIIA or GluRIIB, are expressed at the NMJ. Previously, GluRIIA and GluRIIB complexes were shown to influence NMJ synapse number and strength in an antagonistic way. In this thesis, the role of GluRs and their subunit-specific incorporation in the formation and maturation of PSDs was studied in vivo at developing NMJs.

Following a drastic genetic reduction in the level of all postsynaptic glutamate receptors at the $\mathrm{NMJ}$, PSD maturation was found to be inhibited and proteins normally excluded from PSD membranes remained at these apparently immature sites. However, initial steps of structural postsynaptic assembly proceeded and presynaptic active zones showed normal composition and ultrastructure as well as proper glutamate release. Intriguingly, synaptic transmission as well as glutamate binding to glutamate receptors appeared dispensable for synapse maturation.

In vivo imaging was then used to follow GluRIIA or GluRIIB dynamics during PSD formation and maturation. An essentially irreversible incorporation of GluRIIA was found exclusively during the growth phase of nascent PSDs. Once a sufficient presynaptic glutamate release and postsynaptic conductance through the highly conducting GluRIIA complexes was established at a maturing PSD, further GluRIIA PSD incorporation was blocked, mediated by the cytoplasmic C-terminus of the GluRIIA subunit. In contrast, the incorporation of GluRIIB complexes was reversible and uniform over all PSDs and did not cease with PSD maturation.

This thesis shows that the incorporation of GluRs into PSDs and likely their proteinprotein interactions with further PSD components trigger a conversion from an initial to a mature stage of PSD assembly. Moreover, it is demonstrated that subunitspecific PSD targeting of GluRs also controls the developmental formation and maturation of glutamatergic synapses. Two GluR complexes with opposing 
physiological features compete for PSD incorporation. This competition appears to be necessary to continuously adjust postsynaptic glutamate-mediated conductance and presynaptic glutamate release. In addition, the subunit-specific GluR incorporation seems to determine the final size and physiological performance of individual synapses and with it the collective transmission strength by tuning the overall synapse number per NMJ. 


\section{Introduction}

\subsection{Structure and function of synapses}

The human brain harbors an exquisitely complex neuronal network consisting of $10^{10}$ to $10^{11}$ nerve cells which are interconnected via about $10^{15}$ synaptic contacts. Synapses are highly specialized junctions across which a nerve impulse passes to a neuron, muscle cell or gland cell. Thereby, electrical input signals of the presynaptic cell are rapidly and efficiently transmitted to the connected postsynaptic partner cell. This communication constitutes biological computation which underlies perception, cognition and memory formation.

Despite the high grade of specialization all synapses apply only one of the two basic transmission forms: electrical or chemical. At electrical synapses the transmission is regulated by passive ion flow through tight gap junctions (Fig. 1). Transmission at chemical synapses is mediated by rapid release of a neurotransmitter which triggers an ion influx into the postsynaptic cell.
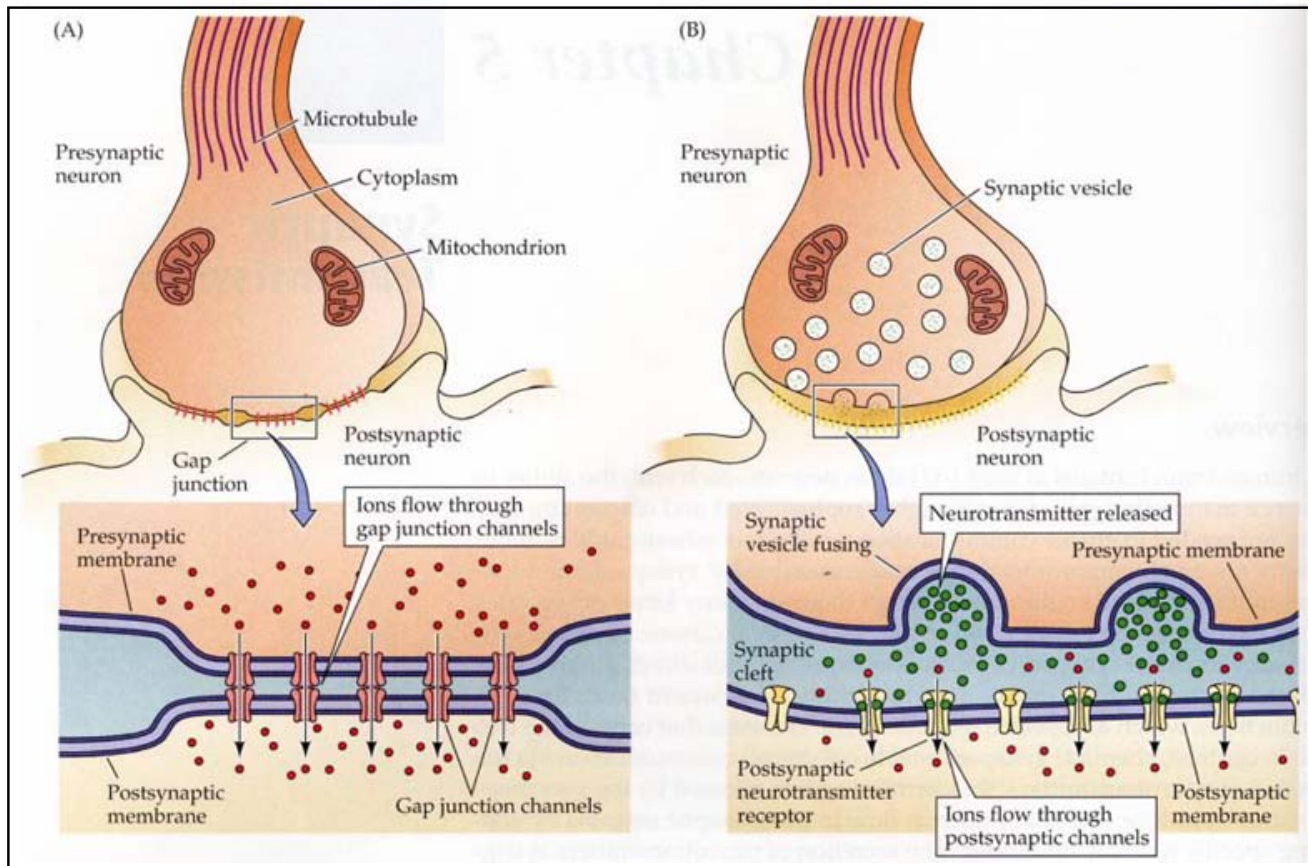

Fig. 1. Electrical and chemical synapse

$\boldsymbol{A}$, Electrical synapse between two neurons. Gap junctions enable the passive direct ion flow from the presynaptic into the postsynaptic neuron. $\boldsymbol{B}$, Chemical synapse. Synaptic vesicles filled with neurotransmitter fuse with the presynaptic plasma membrane and release the neurotransmitter into the synaptic cleft. The postsynaptic membrane harbors ion channels that bind the neurotransmitter, which results in a conformational change that allows ion influx into the postsynaptic cell. Adapted from (Purves et al., 2001).

Following an action potential propagating along the presynaptic neuron, $\mathrm{Ca}^{2+}$ influx into the presynaptic terminal causes the fusion of synaptic vesicles with the presynaptic membrane. Thereby, the neurotransmitter molecules are released from the vesicles into the synaptic cleft. Postsynaptic ion channels specifically bind the 
neurotransmitter. The binding alters the channel conformation and enables the influx of ions, which in turn establishes the signal propagation by changing the membrane potential of the postsynaptic cell (Fig. 1).

Two types of transmission at chemical synapses can be discriminated: excitatory and inhibitory. Excitatory transmission often utilizes the neurotransmitter glutamate, whereas classical inhibitory neurotransmitters are glycine or $\mathrm{y}$-aminobutyric acid (GABA). Neurotransmitters are generally categorized based on their chemical characteristics into four classes: amino acids (glutamate, aspartate, GABA, glycine, acetylcholine), peptides (e.g. vasopressin, somatostasin), monoamines (e.g. dopamine, serotonin) and other neurotransmitters (e.g. nitric oxide, CO).

In the following, three major model systems for studying chemical synapses will be presented. In each case, the general morphological structure and the pre- and postsynaptic constituents of the respective system will be outlined. Furthermore, information about their development and plastic remodeling will be given. Finally, there will be focus on the structure, function and trafficking of ionotropic glutamate receptors, which form the main subject matter of this thesis.

\subsubsection{Vertebrate neuromuscular synapses}

Fig. 2. Vertebrate NMJ Adapted from (Kandel et al., 1991)

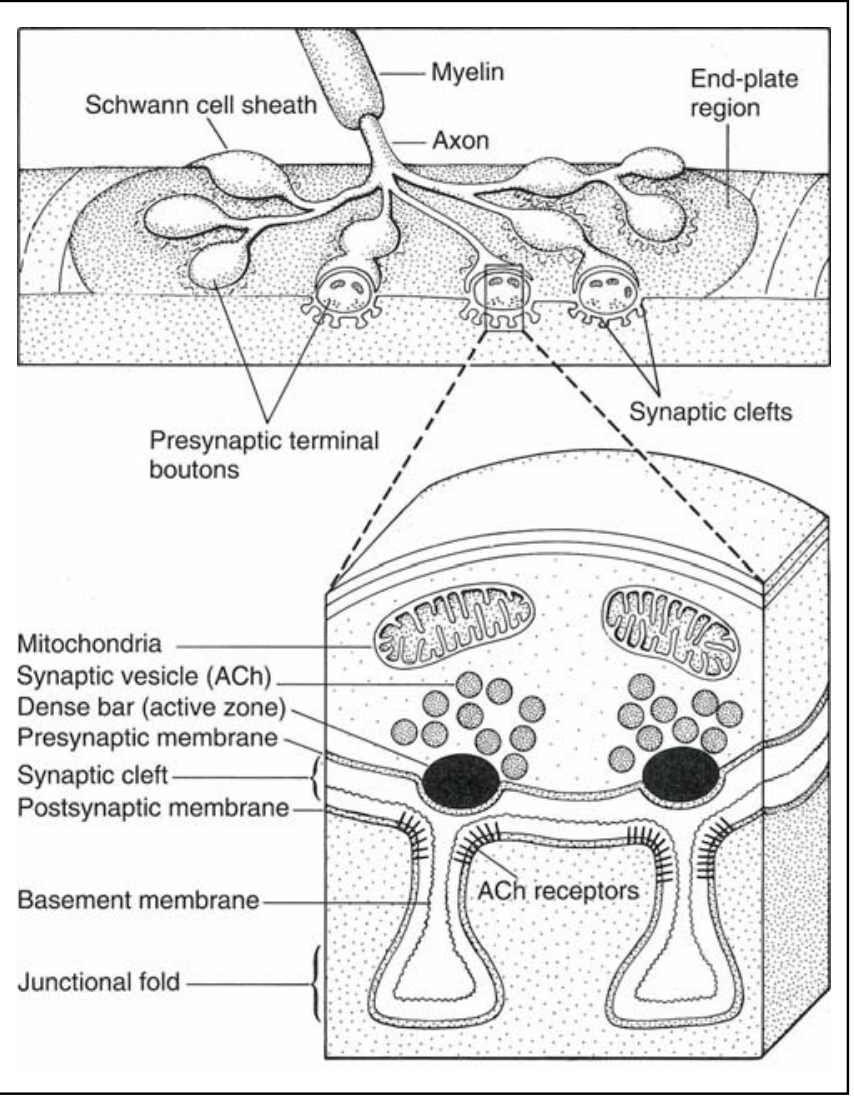


The vertebrate $\mathrm{NMJ}$ forms on a muscle fiber that is innervated by a myelinated axon of a motoneuron. The axon branches into many presynaptic terminal boutons that are ensheathed by Schwann cells (Fig. 2, top). Electrical impulses from the nerve terminals to the muscle are transmitted via the chemical transmitter acetylcholine (ACh). Thereby, each presynaptic bouton contains a dense body called active zone where synaptic vesicles dock and fuse, and which is positioned over a postjunctional fold (deep infolding of the sarcolemma) harboring nicotinic acetylcholine receptors (AChRs). As a specific feature of the vertebrate NMJ synapse, the pre- and postsynaptic membranes are, besides the synaptic cleft, additionally separated by a basal membrane (Fig. 2, bottom).

During muscle innervation, following the initial growth cone contact, AChRs and other synaptic signaling and structural molecules begin to cluster under the overlying nerve terminal (Hughes et al., 2006). Agrin, a heparan sulphate proteoglycan that is released from the motor nerve terminals was shown to be necessary as well as sufficient for AChR clustering. The trans-membrane muscle-specific kinase (MuSK), which is co-localized with AChRs in the postsynaptic membrane, apparently acts as Agrin receptor, although it does not seem to bind to Agrin directly (Sanes and Lichtman, 1999). Muscles of MuSK knockout mice show no signs of postsynaptic differentiation, whereas AChR genes are expressed at normal level (DeChiara et al., 1996).

Another critical factor for AChR clustering downstream of MuSK is the cytoplasmic protein rapsyn, which is precisely co-localized with AChRs. Rapsyn deficient muscles harbor MuSK accumulations but fail to cluster AChRs, which remain atypically, diffusely distributed (Gautam et al., 1995).

Interestingly, it was also shown that the AChRs themselves are required for the clustering of postsynaptic components. Rapsyn clustering failed in zebrafish mutants deficient for AChRs (Ono et al., 2001) as well in C2 myotubes virtually lacking AChRs (Marangi et al., 2001). Moreover, in mice deficient for an adult AChR subunit the postsynaptic membrane was profoundly reorganized and largely missed the typical postjunctional folds. Additionally, several synaptic proteins were abnormally distributed forming atypical protein poor and rich regions (Missias et al., 1997). Hence, the AChRs are not only passive constituents but rather actively involved in the organization of postsynaptic assembly.

\subsubsection{Excitatory vertebrate CNS synapses}

Excitatory synapses in the vertebrate central nervous system are most frequently located on minute lateral dendritic protrusions, the so-called dendritic spines (Fig. 3). 
The main cytoskeleton of the dendritic spines is formed by dynamic Actin filaments that make them capable of rapid morphological changes (Tada and Sheng, 2006). The formation and the morphological changes of spines are meant to play an important role in synaptogenesis and synaptic plasticity (Yuste and Bonhoeffer, 2001; Nikonenko et al., 2002; Matus, 2005).

\section{Fig. 3. Dendritic spines}

Spiny dendrites from a hippocampal pyramidal neuron. Left: light microscope image. Right: reconstruction from serial electron micrographs. Adapted from 6.2C.

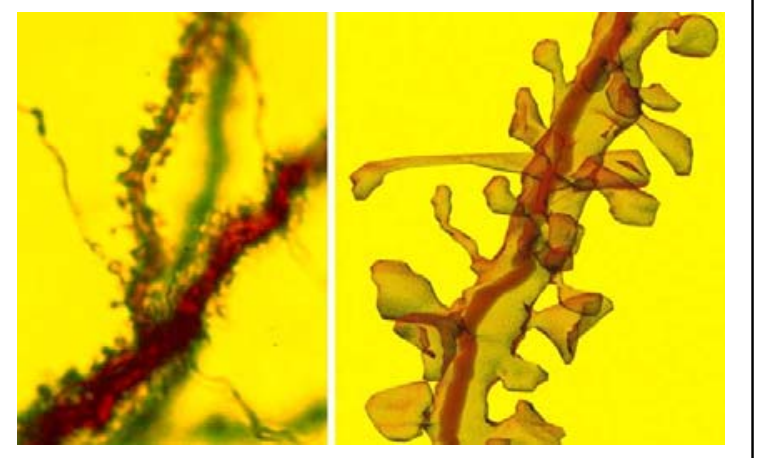

The development of dendritic spines commences with immature dendrites producing motile filopodia that sample the neuropil for presynaptic partners to form synaptic contacts (Fig. 4A). Once an initial contact of the presynaptic axon and the postsynaptic spine is established, structural proteins accumulate at the future synaptic site (Fig. 4B). In the following, spine maturation proceeds by the accumulation of synaptic vesicles in the presynaptic terminal and the integration of glutamate receptors into the postsynaptic membrane (Fig. 4C). Mature spines, which are stable in shape, are typically characterized by an expanded head and a narrow neck (Fig. 3 and Fig. 4D). They vary strongly in size and shape (stubby, thin and mushroom-like).

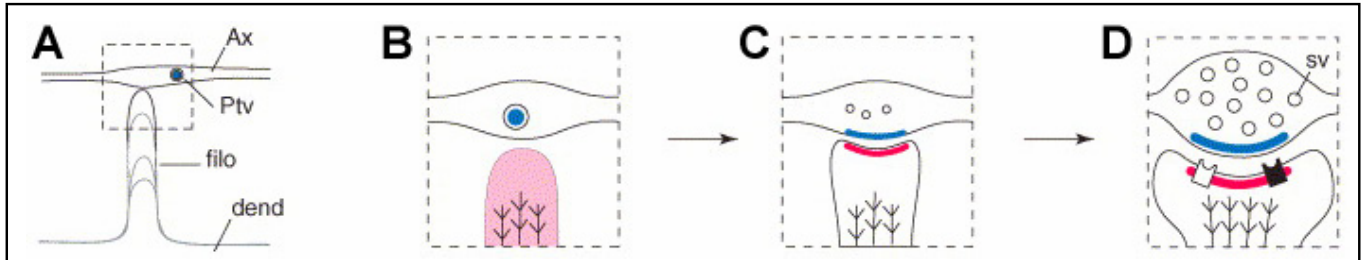

\section{Fig. 4. Model of dendritic spine development}

$\boldsymbol{A}$, Growing dendrites (dend) produce motile filopodia (filo), which extend and retract until a contact to the axon (Ax), containing presynaptic transport vesicles (Ptv), is established. $\boldsymbol{B}$ and $\boldsymbol{C}$, Structural proteins are transported to the presynaptic membrane via Ptvs while the postsynaptic membrane derives from cytoplasmic protein pools. $v$ shapes indicate Actin filaments. $\boldsymbol{D}$, Spine maturation continues with the presynaptic accumulation of synaptic vesicles (sv) and the postsynaptic recruitment of NMDA receptors (black) followed by AMPA receptor (white) incorporation. Modified from (Matus, 2005).

\subsubsection{Presynaptic structure}

When the contact between the presynaptic axon and the postsynaptic dendritic spine has been established, structural proteins are delivered to the nascent presynaptic 
membrane. Thereby, these proteins are transported via presynaptic transport vesicles (Ptvs) as preassembled molecular complexes (Fig. 4A,B) (Ahmari et al., 2000; Shapira et al., 2003). Numerous scaffolding proteins of the active zone as Piccolo, Bassoon, Rim (Rab3-interacting molecule), Liprin-alpha and $\mathrm{N}$-type $\mathrm{Ca}^{2+}$ channels (Ohtsuka et al., 2002; Kim et al., 2003; Shapira et al., 2003) as well as components of the vesicle release machinery were identified on the Ptvs.

Thus, the Ptvs are believed to account for the formation of the active zone itself or the cytomatrix at the active zone (CAZ) (Zhai and Bellen, 2004). The CAZ comprises a network of microfilaments and associated proteins (Zhai and Bellen, 2004) that regulates the translocation of synaptic vesicles to the active zone, the neurotransmitter release and the vesicle endocytosis (Fig. 5) (Ziv and Garner, 2004). The correct targeting of the Ptvs to the nascent presynaptic membrane involves several cell adhesion molecules (CAMs) as Cadherins (Salinas and Price, 2005), Neurexin and Neuroligin (Dean and Dresbach, 2006), but also proteins of the cortical cytoskeleton like Actin and Spectrin.

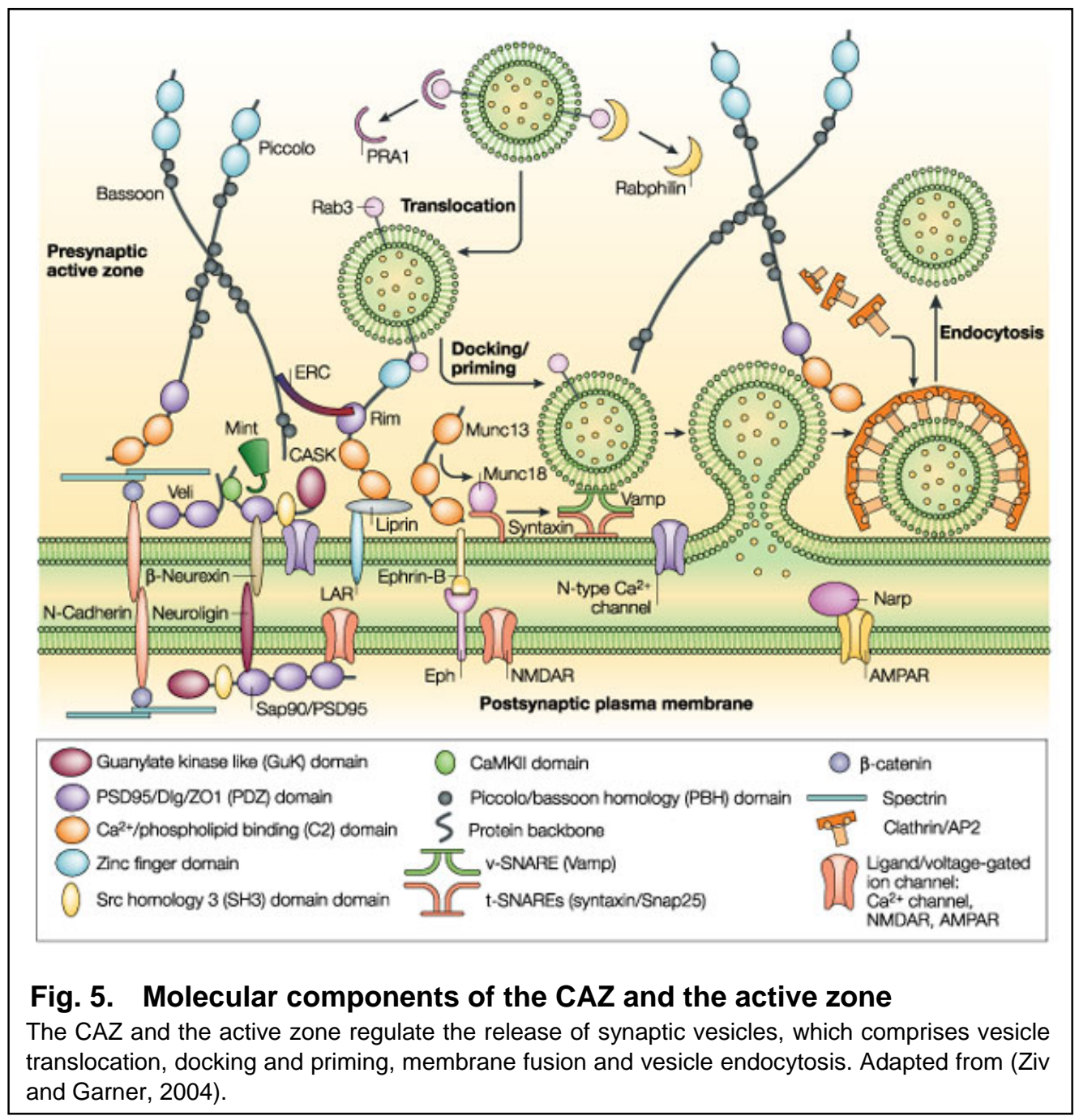




\subsubsection{Postsynaptic structure}

Excitatory synapses in the vertebrate central nervous system are primarily glutamatergic. Following presynaptic vesicle fusion the released glutamate binds to glutamate-sensitive ion channels, which can be subdivided into two groups: metabotropic and ionotropic glutamate receptors. The tetrameric ionotropic glutamate receptor complexes are further categorized in AMPA (alpha-amino-3hydroxy-5-methyl-4-isoxazole-propionic acid), NMDA (N-methyl-D-aspartate) and kainate receptors (for details see 2.2).

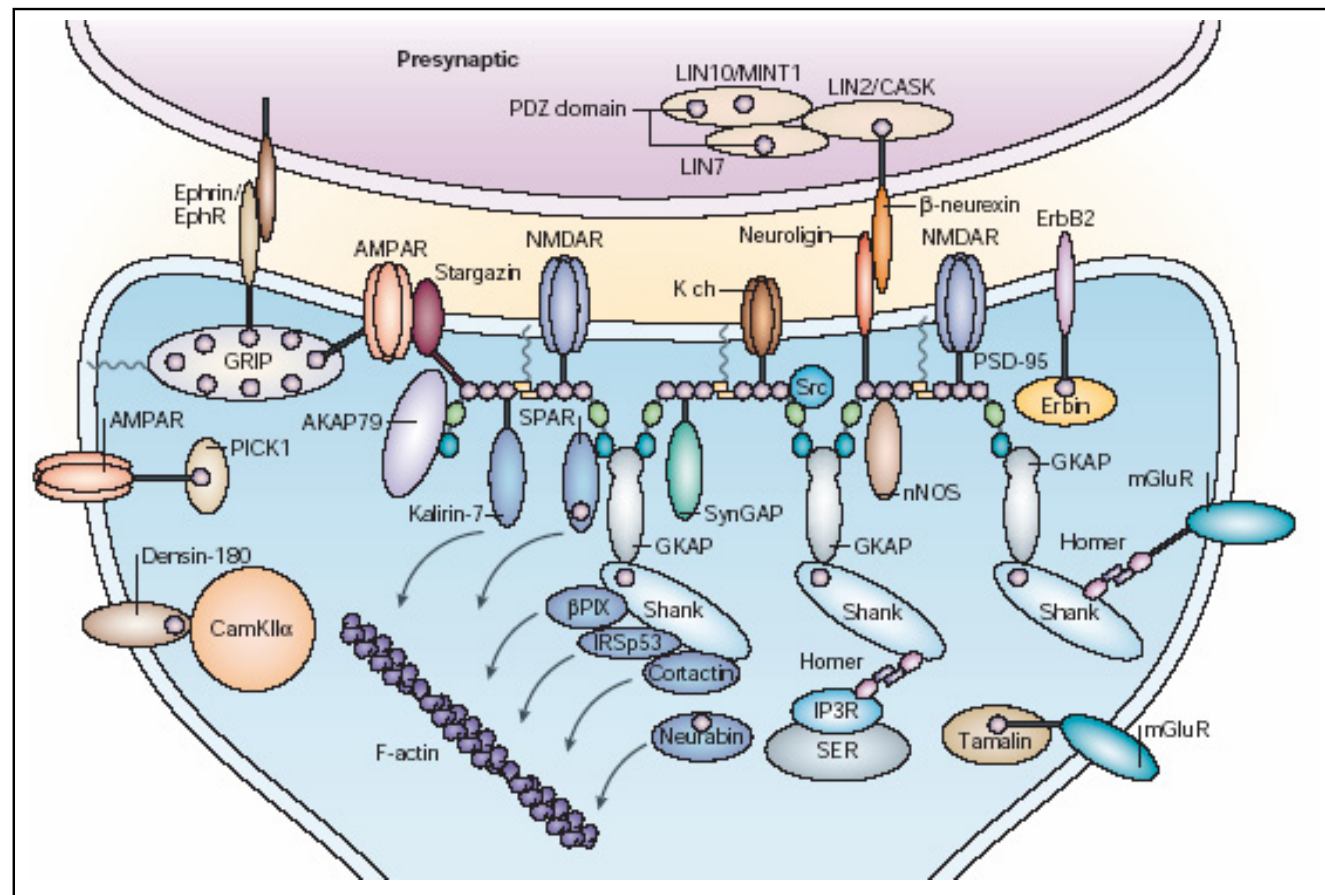

Fig. 6. Molecular components of the postsynaptic density (PSD)

Shown are the main PDZ proteins involved in the organization of the postsynaptic density. PDZ domains are indicated by small purple circles, the C-terminal cytoplasmic tails of membrane proteins by black lines. Abbreviations denote: AKAP79, A-kinase anchor protein 79; AMPAR, AMPA ( $\alpha$-amino-3-hydroxy-5-methyl-4-isoxazole propionic acid) receptor; $\beta P I X$, PAAK-interactive exchange factor; CaMKIIa, $\alpha$-subunit of $\mathrm{Ca}^{2+1}$ calmodulin-dependent protein kinase II; GK, guanylate kinase-like domain; EphR, ephrin receptor; ErbB2, EGF-related peptide receptor; GKAP, guanylate kinase-associated protein; GRIP, glutamate-receptor-interacting protein; IP3R, IP3 receptor; IRSp53, insulin-receptor substrate p53; K ch, potassium channel; LIN7, lin7 homologue; LIN10, lin10 homologue; mGluR, metabotropic glutamate receptor; NMDAR, NMDA ( $N$-methyl-D-aspartate) receptor; nNOS, neuronal nitric oxide synthase; PICK1, protein interacting with C kinase 1; PSD-95, postsynaptic density protein 95; SER, smooth endoplasmic reticulum; SH3, Src homology 3 domain; Shank, SH3 and ankyrin repeat-containing protein; SPAR, spine-associated RapGAP; SynGAP, synaptic Ras GTPase-activating protein. Adapted from (Kim and Sheng, 2004).

The glutamatergic transmission is supported by a specialized postsynaptic subcellular organization, called the postsynaptic density (PSD). The PSD is involved in clustering and anchoring of postsynaptic receptors and ion channels and contains a specialized submembranous cytoskeleton with a rich collection of proteins that 
serve to organize this membrane specialization (Fig. 6) (Kim and Sheng, 2004). In contrast to the assembly of presynaptic active zones involving the delivery of prefabricated transport packets (see 2.1.2.1), postsynaptic assembly seems to depend on gradual de novo clustering of component proteins (Bresler et al., 2004). Synaptic non-NMDA receptors may either be recruited into PSDs from a diffuse plasma membrane pool by lateral migration (Borgdorff and Choquet, 2002) or be incorporated via subunit specific constitutive or activity-dependent pathways (Bredt and Nicoll, 2003), potentially using preformed slots established at the postsynaptic membrane (Barry and Ziff, 2002). Moreover, postsynaptic glutamate receptor levels are regulated by various adaptor proteins, kinases and scaffolding molecules (McGee and Bredt, 2003). Within the PSD, scaffolding proteins containing one or more PDZ domain are highly abundant (Fig. 6) (Walikonis et al., 2000). Among them are PSD-95 (postsynaptic density protein 95) and SAP97 (synapse-associated protein 97), both membrane-associated guanylate kinases (MAGUKs), GRIP (glutamate receptor interacting protein), ABP (AMPA receptor binding protein) and PICK1 (protein interacting with C kinase).

\subsubsection{Structural synaptic plasticity and LTP}

In 1949 Hebb postulated that two mechanisms were responsible for memory storage in the brain: alteration in synaptic strength and formation of novel synapses (Hebb, 1949). 24 years later, Bliss and Lomo developed a first paradigm of brief tetanic stimulation on hippocampal neurons to produce a long lasting form of synaptic plasticity which can last for hours or days, named long-term potentiation (LTP) (Bliss and Lomo, 1973). Since then, LTP was and is widely used as a cellular model for information storage at central synapses.

To establish LTP, various structural synaptic changes are conceivable. On the one hand, LTP could result from structural alterations of pre-existing synapses like the conversion from a non-functional (silent) to a functional state, synapse splitting or strengthening of pre- and/or postsynaptic structures. On the other hand, the establishment of novel synaptic contacts and thereby an increase in the overall synapse number could underlie LTP. Moreover, as dendritic spines are very mobile, changes in the spine number and morphology could as well be involved.

In fact, various changes in spine morphology can be observed following LTP induction (Fig. 7) (Yuste and Bonhoeffer, 2001; Nikonenko et al., 2002). Within the first minutes after potentiation the postsynaptic receptor composition as well as the presynaptic release properties are modified, while obvious morphological changes are not detectable (Fig. 7A). 30min after LTP induction, the swelling of spine heads 
and a parallel increase in the synaptic area can be demonstrated (Fig. 7B). Moreover, perforated synapses (Fig. 7C) and bifurcating spines can be observed (Fig. 7D). Finally, new spines harboring novel synapses can emerge close to the activated spines (Fig. 7E) (Yuste and Bonhoeffer, 2001).

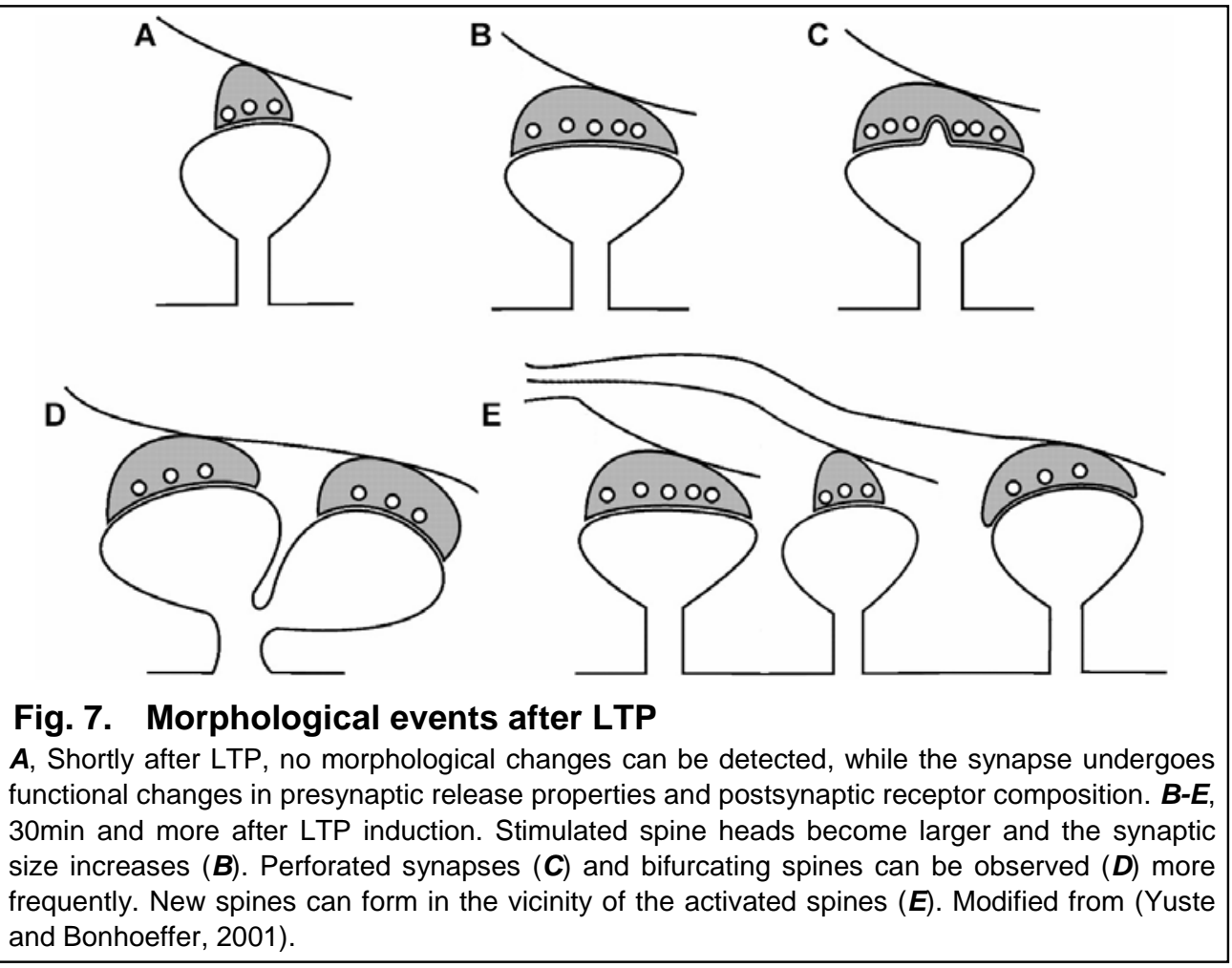

However, recent evidence suggests that the bifurcating spines (sdMSBs: samedendrite, multiple-synapse boutons) do not arise from synapse and spine splitting as mature dendrites and axons were found passing through the gaps between the spine pairs (Fiala et al., 2002).

Another additional contribution to the early phase of LTP (Fig. 7A) might be the activation of silent synapses. A recent study suggests that the conversion of silent synapses to an activated state occurs 3-6h after stimulation whereas the addition of novel synapses occurred $12-18 \mathrm{~h}$ after stimulation and might be therefore important for the late phase of LTP (Kim et al., 2003).

\subsubsection{Drosophila neuromuscular synapses}

\subsubsection{Drosophila melanogaster as a model system}

The fruit fly Drosophila melanogaster has been used as a genetic model system for almost a century. Despite its small genome of only $165 \mathrm{Mbp}$ distributed to four chromosome pairs, most Drosophila genes (about 14000 in total) are evolutionary conserved to vertebrates. One of the main advantages of Drosophila is its short life 
cycle of about 10 days at $25^{\circ} \mathrm{C}$ (Fig. 8). Furthermore, the establishment and application of various transgenic and knockout strategies is simplified compared to vertebrates. Additionally, the UAS/Gal4 system allows the ectopical and temporally defined expression of a gene of interest (Brand and Perrimon, 1993). Morphologically, most developmental stages are easily accessible with a huge variety of physiological, histological and microscopic techniques. In result, this allows vast functional in vivo analysis of cellular mechanisms.

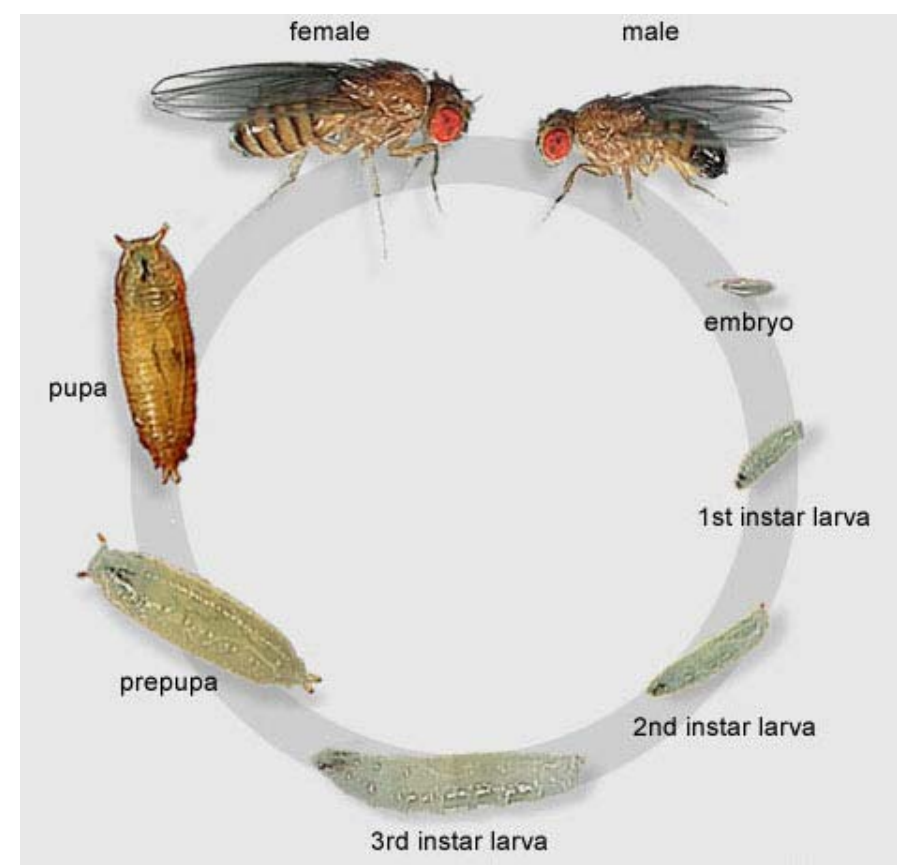

\section{Fig. 8. Drosophila life cycle}

After cellularization of the blastoderm, gastrulation, germ band elongation and retraction the embryo hatches about $24 \mathrm{~h}$ after the egg laying (at $25^{\circ} \mathrm{C}$ ). The following $1^{\text {st }}$ and $2^{\text {nd }}$ instar larval stages last again about one day each and end with the molt of the larva. After another two days the $3^{\text {rd }}$ instar larvae reach the wandering stage, which is followed by the pupation. The subsequent metamorphosis takes three days and is finished with the eclosion and the hatching of the adult fly. The presented image originates from $6.2 \mathrm{~A}$.

\subsubsection{Properties of the Drosophila NMJ}

The neuromuscular junction of Drosophila (NMJ) allows efficient genetic analysis of development, plasticity and function of glutamatergic synapses (Jan and Jan, 1976; Keshishian et al., 1996; Prokop, 1999; Koh et al., 2000; Richmond and Broadie, 2002). Basic features of its synaptic function and the majority of synapse-associated proteins are evolutionary conserved with excitatory CNS synapses (see 2.1.2).

The NMJ comprises a segmentally repeated set of 30 abdominal muscle cells (Fig. 9) (Bate et al., 1999), which is innervated by 36 identified motoneurons per hemisphere branching into presynaptic varicosities (boutons) (Fig. 10) (Landgraf and Thor, 2006). 
Fig. 9. Drosophila larval body wall muscles

Illustrated is the segmentally repeated set of 30 abdominal muscles. Muscles that were of relevance in this study are: ventral-longitudinal muscles 4,6 , 7, 12 and 13; ventral-oblique muscle 14; ventral-acute muscle 27. The upper panel presents a dorsal, the lower a ventral view. Adapted from (Bate et al., 1999).

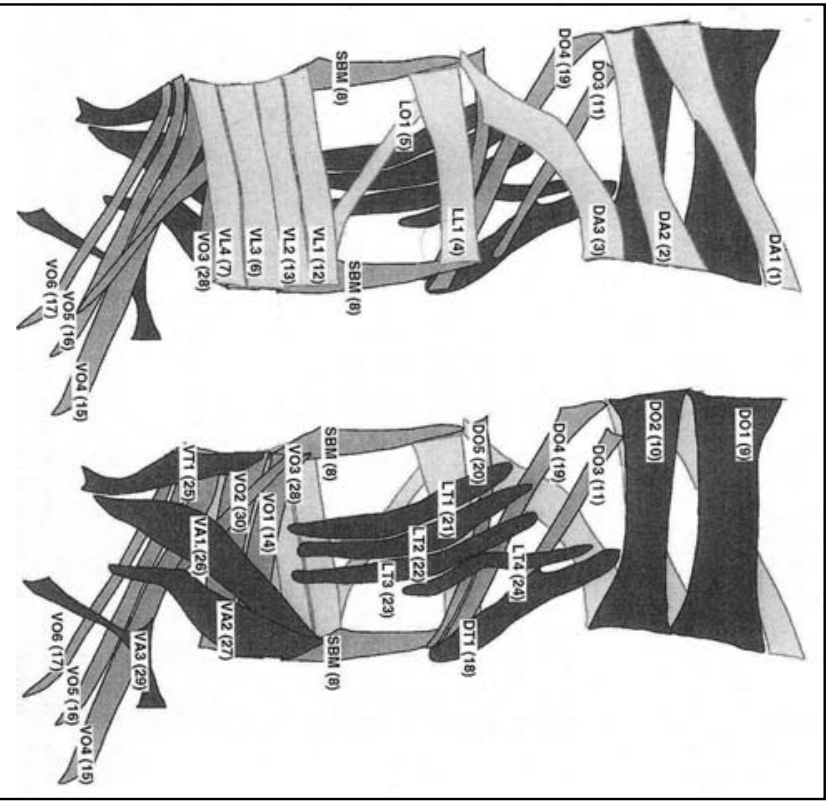

Thereby, three innervation types called type I, II and III exist. Type I presynaptic boutons (Fig. 10), which account for the excitation of the postsynaptic muscle cell, are exclusively glutamatergic and further subdivided into Is and Ib boutons. While Is boutons have a diameter of $1-3 \mu \mathrm{m}$, lb boutons reach a diameter of 3-5 $\mathrm{mm}$. Each $\mathrm{lb}$ bouton harbors about 10-20 synapses (Atwood et al., 1993). Type II and III innervations, which were not addressed in this study, have not been associated with an electrically observable postsynaptic response so far (Rheuben et al., 1999).

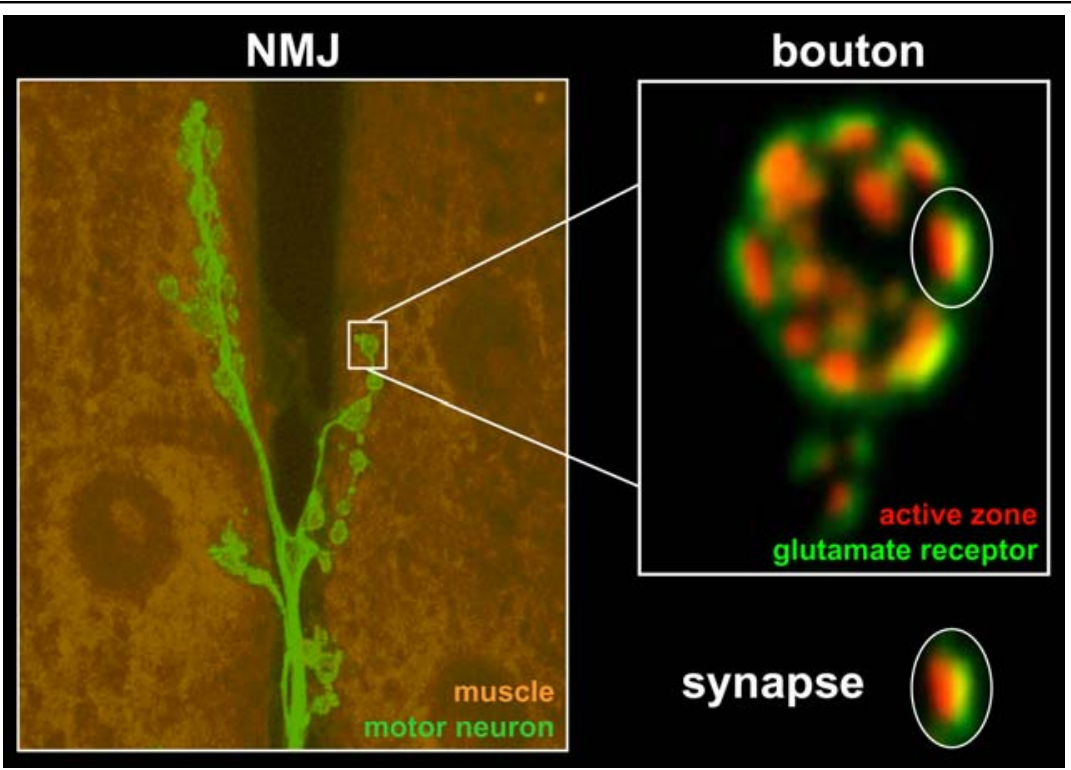

Fig. 10. NMJ - bouton - synapse

Shown are the ventral-longitudinal muscles 6 and 7 (orange) innervated by a motoneuron (green) branching into numerous varicosities called boutons (left panel). One bouton (type lb) comprises 10-20 synapses that are characterized by postsynaptic glutamate receptors opposing the presynaptic active zone (right panel). Partially modified from J Neurosci, Vol.24 (2004). 


\subsubsection{Development of the Drosophila NMJ}

The embryonic development of the neuromuscular junction (Fig. 11) can be separated in three stages, namely the growth cone stage, the prevaricosity stage and the varicosity stage. During the growth cone stage (13 to 16 hours after egg laying, $A E L)$, filopodia sent out from the motoneuron growth cone contact muscle myopodia sent out from the future innervation site (Ritzenthaler et al., 2000). Thereby, inappropriate contacts are again withdrawn (Broadie and Bate, 1993). The prevaricosity stage (16h AEL) is characterized by the enlargement of the central region of the growth cone (at the nerve entry point into the muscle) and the formation of distinct branches. During the final varicosity stage (17h AEL) distinct varicosities form from the general swelling of the prevaricosity (Rheuben et al., 1999).

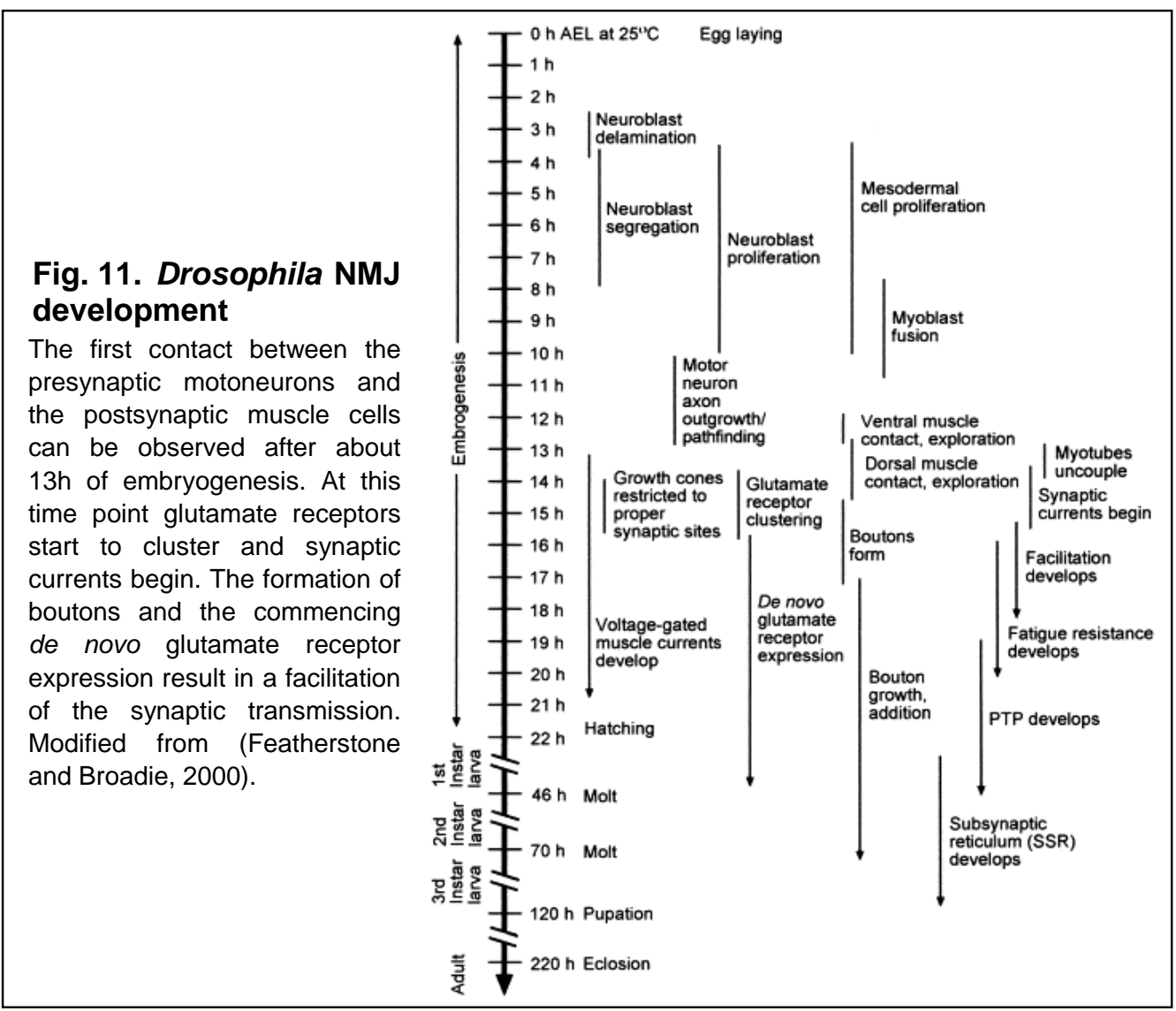

The initial neuromuscular contact requires stabilization mediated by several cell adhesion molecules. Among them is Fasciclinll II (FasII), which is related to vertebrate NCAMs (neuronal cell adhesion molecules). It is initially strongly expressed on the surface of the innervating motoneurons and at low levels also in the muscle cell (Schuster et al., 1996). When the neuromuscular connection is established Fasll is clustered at both pre- and postsynaptic membrane. The further maintenance of Fasll at the neuromuscular synapses is largely mediated by Discs 
large (Dlg), which is assigned to the PSD-95-type MAGUK family (Thomas et al., 1997; Zito et al., 1997).

In contrast to vertebrate NMJs where Agrin secretion from the nerve terminal initiates the clustering of postsynaptic proteins, no Agrin homologue seems to be present at the Drosophila NMJ. Nevertheless, recent studies showed that molecules such as Wnt and TGF $\beta$ (transforming growth factor $\beta$ ), which act during embryo morphogenesis, are also involved in the differentiation of synapses (Packard et al., 2003).

While the presynaptic assembly of active zones can take place independent of the muscles (Prokop et al., 1996), the postsynaptic clustering of glutamate receptors requires and starts with the initial axon-muscle contact (Broadie and Bate, 1993). Notably, complete suppression of neurotransmission does not inhibit the formation of glutamate receptor clusters at the postsynaptic membrane (Featherstone and Broadie, 2000). Consistently, it could be demonstrated that embryonic synapse assembly remained apparently unaltered in Munc-13 or Munc-18 null mutant mice lacking any neurotransmission (Verhage et al., 2000; Varoqueaux et al., 2002).

\subsubsection{Glutamatergic NMJ synapses}

The principle structure of glutamatergic synapses at the Drosophila NMJ is similar to excitatory vertebrate CNS synapses (see 2.1.2). The synaptic ultrastructure of Drosophila NMJ synapses (visualized by transmission electron microscopy in Fig. 12 ) is characterized by a close apposition and a high electron density of the pre- and postsynaptic membranes over several hundred nanometers (synaptic cleft span: 10$20 \mathrm{~nm}$ ). Moreover, presynaptic active zones are typically associated with electrondense specializations (T-bars) (Atwood et al., 1993; Zhai and Bellen, 2004) which are required for the efficient release of synaptic vesicles (Kittel et al., 2006). The postsynaptic density juxtaposed to the active zone provides the clustering of glutamate receptors, voltage-gated ion channels, scaffolding and regulatory molecules as PAK (p21-activated kinase) (Albin and Davis, 2004; Qin et al., 2005; Prokop and Meinertzhagen, 2006). Individual synapses are surrounded by the perisynaptic region which harbors adhesion proteins as Fasll, which is linked to synaptic stabilization and growth (Schuster et al., 1996; Sone et al., 2000). Beneath the PSD the muscle membrane is highly convoluted forming the subsynaptic reticulum (SSR). Various scaffolding and adhesion proteins as Dlg, which might play a role in the structural organization and signaling mechanisms of cell adhesion molecules and ion channels, are found at the SSR membrane (Thomas et al., 1997). 


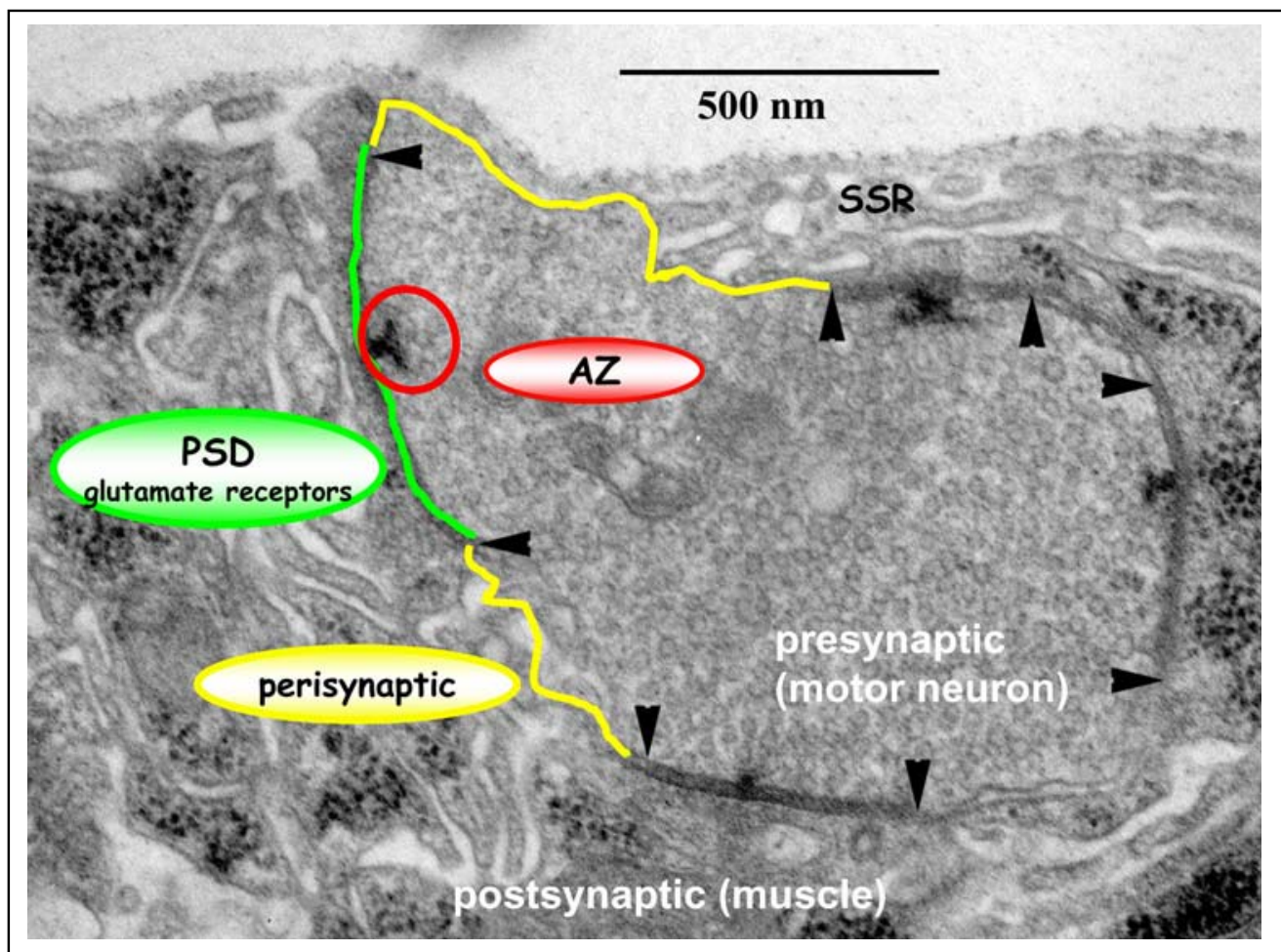

Fig. 12. Ultrastructure of glutamatergic Drosophila NMJ synapses

Shown is an electron microscopic cross-section through a synaptic bouton (filled with synaptic vesicles). The presynaptic active zone (red), typically characterized by the presence of an electron-dense specialization ( $T$-bar), is in close coordination with the postsynaptic density (green) containing the glutamate receptors. Pre- and postsynaptic membranes, which are separated by a narrow synaptic cleft (10-20nm), show linear apposition and a high electron density along the synaptic region (marked by arrowheads). The perisynaptic region (yellow) surrounds the synapses. The subsynaptic reticulum (indicated as SSR) forms by pronounced folding of the muscle membrane.

\subsubsection{Activity-dependent remodeling}

Several mutants which suppress or enhance the outgrowth of the Drosophila NMJ have been identified. As mentioned before the cell adhesion molecule Fasll mediates synaptic stabilization and growth (Schuster et al., 1996). It was shown that increased neuronal activity decreases synaptic Fasll levels, and moreover, genetic Fasll reduction increased the number of synapses (Schuster et al., 1996). Therefore, a weakened cell adhesion might be a prerequisite for the addition of novel synaptic contacts following activity enhancement. An elevated presynaptic activity could be genetically achieved by establishing a double mutant situation for both the Eag (ether a go-go) and Shaker (Sh) potassium channel leading to an increased frequency of nerve-evoked action potentials. This in turn resulted in elevated CAMP levels and finally enhanced morphological NMJ outgrowth (Zhong et al., 1992). The involvement of CAMP signaling in NMJ plasticity could be confirmed using the learning mutant dunce (Dudai et al., 1976; Zhong et al., 1992; Cheung et al., 1999), which lacks a cAMP phosphodiesterase and therefore accumulates CAMP (Davis 
and Kauvar, 1984; Zhong et al., 1992). NMJ outgrowth could be inhibited by a concomitant knockout of rutabaga, which encodes for the adenyl cyclase (Dudai and Zvi, 1985; Livingstone, 1985). Alongside with the effects on NMJ morphology, cAMP signaling also plays a role in the regulation of synaptic dimensions and structure. Whereas a genetic elimination of rutabaga caused a dramatic increase in synapse size but a decrease in synapse number (Renger et al., 2000; Shayan and Atwood, 2000), dunce mutant synapses were not different from controls (Renger et al., 2000). In turn, presynaptic overexpression of dunce mimicked the rutabaga knockout (Shayan and Atwood, 2000).

A modulation of neural activity without genetic manipulation can be achieved through chronic larval rearing at an increased temperature of $29^{\circ} \mathrm{C}$, which enhances their locomotion (Sigrist et al., 2003; Zhong and Wu, 2004). The elevated locomotion resulted in increased arborization of the $\mathrm{NMJ}$ and a boost of bouton and synapse number (Sigrist et al., 2003; Zhong and Wu, 2004)

Besides the alterations of presynaptic neural activity, changes in glutamate receptor complex composition are implicated in long-term changes of synaptic structure and transmission (Petersen et al., 1997; DiAntonio et al., 1999; Sigrist et al., 2002). Thereby, increased expression of the glutamate receptor subunit GluRIIA (see 2.2.3) was shown to elevate the number of synapses forming per NMJ (Sigrist et al., 2002).

\subsection{Structure and function of ionotropic glutamate receptors}

\subsubsection{Structure and assembly of non-NMDA glutamate receptors}

Glutamate receptors can be subdivided into two groups, namely metabotropic GluRs, which are coupled to G-proteins, and ionotropic GluRs. Ionotropic glutamate receptors (iGluRs) mediate excitatory synaptic transmission at most mammalian CNS synapses. They are discriminated in AMPA, NMDA and Kainate receptors. Non-NMDA receptors form homo- or heterooligomers, most likely tetramers (Rosenmund et al., 1998), composed of the subunits GluR1-4 (AMPA type) and GluR5-7 and/or KA1-2 (Kainate type) (Madden, 2002). A typical glutamate receptor subunit consists of an amino terminal domain (NTD), a ligand binding domain (S1/S2; for molecular 3D structure see Fig. 58), three transmembrane domains, a reentrant pore loop and an intracellular C-terminal domain (Fig. 13, Fig. 20). The NTD, which covers about half of the protein, contributes to receptor assembly (Ayalon and Stern-Bach, 2001). Besides the ligand binding, the S1/S2 domain controls the channel closure, thereby determines the activation and desensitization behavior of the ion channel, and is involved in the dimerization and tetramerization of subunits (see Fig. 59, Fig. 60). The reentrant pore loop, which is located between the 
first and second transmembrane domain, lines the channel and controls the ion gating (Madden, 2002). The C-terminal domain is involved in trafficking and transport of the receptor subunits (see 2.2.2) (Malinow and Malenka, 2002).

The assembly of the tetrameric non-NMDA glutamate receptor complexes is thought to follow the 'dimer-of-dimers' model (Fig. 13). Subunit monomers associate primarily through interactions between their amino terminal domains. The formed dimers undergo a secondary dimerization, mainly mediated by interactions between the transmembrane domains and the S2 ligand binding domain (Fig. 59) (Ayalon and Stern-Bach, 2001; Madden, 2002). The receptor assembly is taking place in the endoplasmatic reticulum (ER). The preassembled complexes are sorted in the Golgi and trans-Golgi apparatus into transport vesicles and handled to the synapses (Malinow and Malenka, 2002).

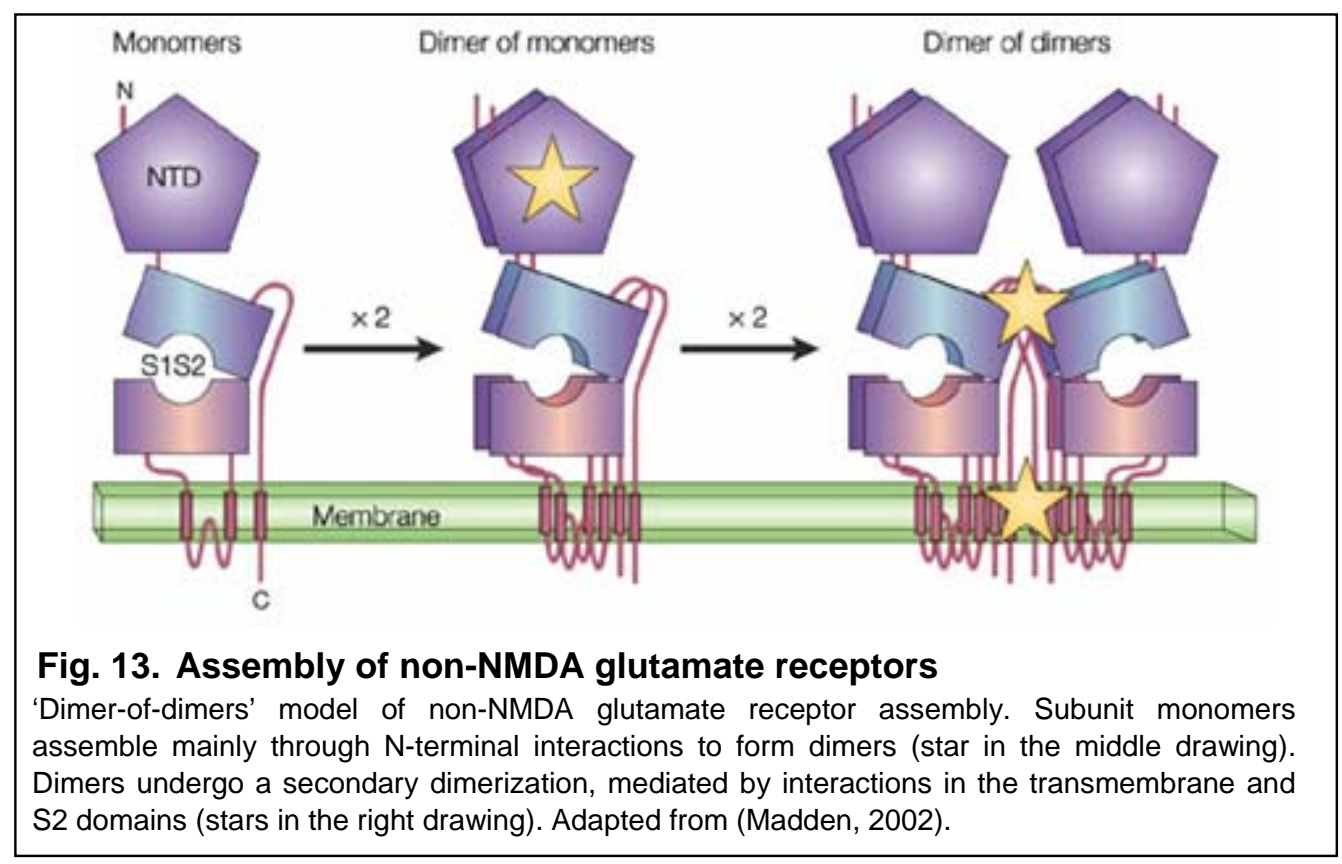

\subsubsection{Glutamate receptor trafficking}

Long-term potentiation (LTP) and the converse long-term depression (LTD) are associated with persistent structural and functional modifications of synapses (see 2.1.2.3). Thereby, the regulated trafficking of AMPA receptor to and integration into the postsynaptic membrane plays an important role (Malinow and Malenka, 2002). Following LTP, silent synapses, which are characterized by having only NMDA receptors can be converted to active synapses by the rapid and specific functional recruitment of AMPA receptors (Fig. 14, top) (Isaac, 2003). This modulation of synaptic AMPA receptor number might involve cytoplasmic receptor-bearing vesicles and exocytotic mechanisms (Malinow and Malenka, 2002). In contrast, endocytosis 
of AMPA receptors via clathrin-coated vesicles contributes to LTD (Carroll et al., 1999).

How is the AMPA receptor recruitment and drawback organized? Various, mostly Cterminal molecular interactions of AMPA receptors contributing to AMPA receptor trafficking and transport have been determined. Thereby, mostly interactions with PDZ proteins (among them: GRIP1, ABP/GRIP2, PICK1, SAP97) are of relevance (Malinow and Malenka, 2002; Kim and Sheng, 2004). Besides that, other proteins (as RIL and Band $4.1 \mathrm{~N}$ ) link the receptor complexes to the Actin cytoskeleton. In addition to that, the ATPase NSF (N-ethylmaleimide-sensitive factor), known to play a role in synaptic vesicle exocytosis (Whiteheart et al., 1994), interacts with the cytoplasmic tail of GluR2 (Nishimune et al., 1998). NSF has been reported to increase surface expression of AMPA receptors (Luscher et al., 1999) and to make them resistant to endocytosis (Shi et al., 2001).

The PDZ proteins GRIP and ABP are part of the scaffolding matrix and likely serve various functions in the delivery, stabilization and endocytosis of AMPA receptors (Malinow and Malenka, 2002). As GRIP and ABP, PICK1 interacts with a PDZ domain within the $\mathrm{C}$-terminal region of GluR2 and contributes to AMPA receptor clustering (Xia et al., 1999). The interaction of SAP97 with the absolute C-terminus of GluR1 can be detected early in the secretory pathway and might be of importance for the trafficking of GluR1/2 complexes (Sans et al., 2001). Recent evidence suggested, that also Stargazin, an AMPA receptor associated transmembrane protein which interacts with the PSD-95 scaffold protein, is required for surface and synaptic expression of AMPA receptors (Chen et al., 2000; Tomita et al., 2005).

The delivery of AMPA receptors to the plasma membrane also depends on receptor phosphorylation. The phosphorylation of Ser831 on GluR1 by calmodulin-dependent kinase II (CamKII) leads to increased receptor conductance during LTP but is not required for AMPA receptor transport (Hayashi et al., 2000). Different from that, phosphorylation of Ser845 on GluR1 by protein kinase A (PKA) is necessary for synaptic delivery of GluR1-containing complexes (Malinow and Malenka, 2002). GluR2 phosphorylation on Ser880 (part of the C-terminal PDZ domain) by protein kinase C (PKC) strongly decreases the affinity of GluR2 for GRIP and ABP but not for PICK1, resulting in increased receptor internalization (Chung et al., 2000).

The specific molecular interactions of AMPA receptor subunits are the basis for the subunit-dependent AMPA receptor insertion into the postsynaptic membrane. In the most extensively studied adult rat hippocampus, assembly of AMPA receptors primarily involves heteromerization of GluR1 with GluR2, or GluR2 with GluR3 (Wenthold et al., 1996). Recent work has deciphered two general mechanisms of 
postsynaptic AMPA receptor trafficking (Shi, 2001). Following LTP induction, GluR1/2 complexes are inserted de novo in an activity-dependent manner, thereby supporting transmission strengthening. In contrast, GluR2/3 complexes undergo a constitutive recycling largely independent of activity (Fig. 14) (Shi, 2001; Barry and Ziff, 2002)

Fig. 14. Subunit-dependent AMPA receptor insertion and recycling

Following LTP, at both silent and active synapses GluR1/2 complexes are inserted into the postsynaptic membrane resulting in dominance of the GluR1 subunit over GluR2. During or following insertion, a molecular placeholder (slot protein) may also be added to the synapse. Thereby, during constitutive recycling of GluR2/3 complexes the synapse might have a 'memory' of how many AMPA receptors may reside at the membrane. Adapted from (Barry and Ziff, 2002).

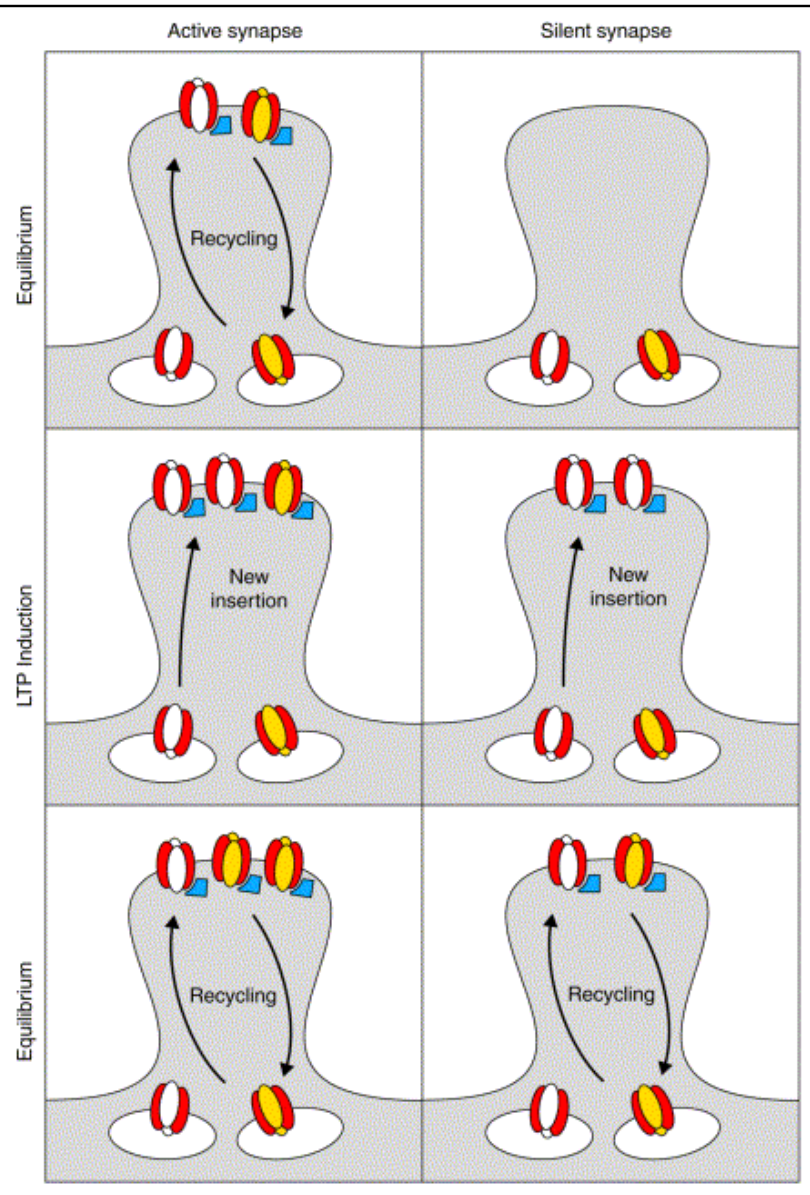

GluR1/2 heteromer

GluR2/3 heteromer

Slot protein

\subsubsection{Glutamate receptors at Drosophila NMJ synapses}

The glutamate receptor subunits expressed at the Drosophila NMJ are related to mammalian non-NMDA type glutamate receptors. So far, five different glutamate receptor subunits (namely GluRIIA, IIB, IIC, IID and IIE) have been identified (Schuster et al., 1991; Petersen et al., 1997; Marrus et al., 2004; Featherstone et al., 2005; Qin et al., 2005). Thereby, genetic considerations suggested that the most likely tetrameric receptor complexes are formed by incorporating GluRIIC, GluRIID and GluRIIE with either GluRIIA or GluRIIB (Qin et al., 2005). The two receptor complexes, which co-exist within individual PSDs, are redundant for NMJ synapse formation (Petersen et al., 1997; DiAntonio et al., 1999; Marrus et al., 2004; Chen et 
al., 2005; Qin et al., 2005). gluRIIC $C^{\text {null }}$, gluRIID ${ }^{\text {null }}$ and gluRIIE ${ }^{\text {null }}$ single mutants, as

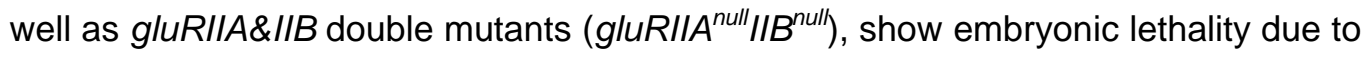
the absence of all synaptic glutamate receptors (Petersen et al., 1997; Featherstone and Broadie, 2002; Marrus et al., 2004; Qin et al., 2005).

GluRIIA and GluRIIB containing complexes differ fundamentally in their physiological properties. GluRIIB complexes show an about 10-fold faster desensitization than GluRIIA complexes, however an identical single-channel amplitude (Fig. 15) (DiAntonio et al., 1999). Accordingly, GluRIIA complexes account for the lion's share of synaptic currents at the Drosophila NMJ. In contrast to gluRIIB mutants, gluRIIA mutants are characterized by a strongly decreased quantal size (miniature evoked junctional current, $\mathrm{mEJC}$ ) and concomitant compensatory increase in quantal content (Petersen et al., 1997; DiAntonio et al., 1999) This retrograde increase of presynaptic release (Davis et al., 1998) goes along with structural alterations like a smaller presynaptic terminal area with increased density of T-bars at active zones (Reiff et al., 2002).

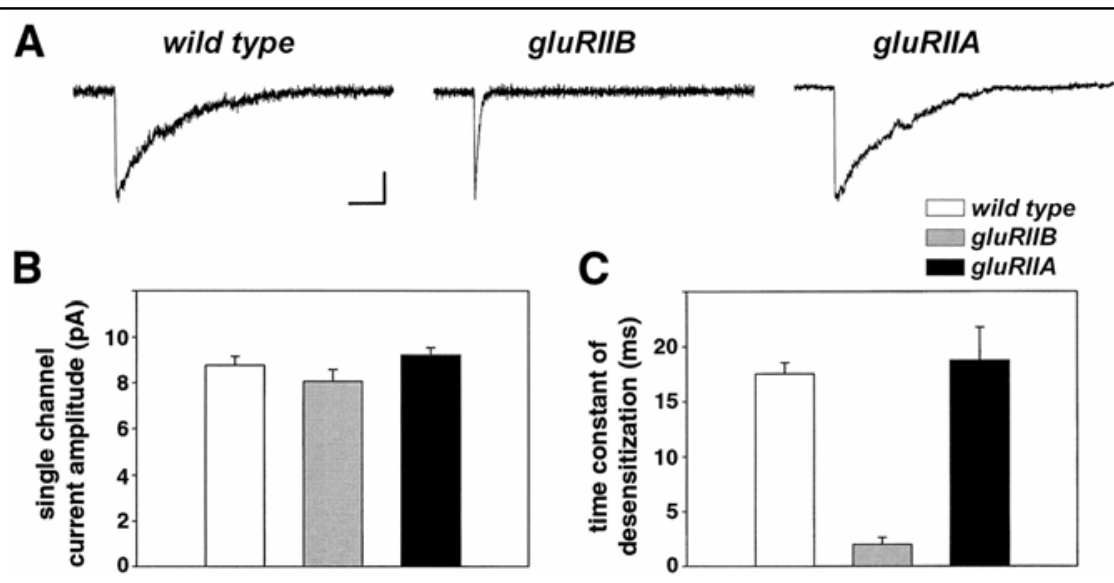

Fig. 15. Receptor subunit composition regulates desensitization kinetics Outside-out patches were isolated from the larval muscle membrane of wild type and animals rescued by the expression of gluRIIA or gluRIIB in the gluRIIA $A^{\text {null }} / B^{\text {null }}$ background. The traces show average responses $(\boldsymbol{A})$ to the rapid application of $10 \mathrm{mM}$ glutamate. The single-channel current amplitude $(\boldsymbol{B})$ was not significantly different in the three genotypes, but the time constant of desensitization $(\boldsymbol{C})$ was much more rapid in gluRIIB larvae $(p<0.005)$. Calibration: $10 \mathrm{msec}$, 5pA. Modified from (DiAntonio et al., 1999).

The synaptic glutamate receptor level can be regulated by PSD-associated proteins as the p21-associated kinase (PAK) (Sone et al., 2000; Albin and Davis, 2004) or the cell adhesion molecule Fasll (Schuster et al., 1996). Moreover, non-vesicular glutamate release can affect glutamate receptor levels at the PSD (Featherstone et al., 2002).

The molecular mechanisms underlying the subunit-specific synaptic trafficking and anchoring of GluRIIA and GluRIIB complexes are widely unknown. However, recent 
studies imply that the two receptor subtypes are likely differentially linked with membrane-associated proteins, scaffolding components or signaling molecules. GluRIIB complexes were shown to be specifically stabilized by the MAGUK Dlg (Chen and Featherstone, 2005), while GluRIIA complexes are anchored at the PSD by Coracle, a homolog to the mammalian Band 4.1N protein (Chen et al., 2005). Additionally, GluRIIA expression could be controlled by local translation (Sigrist et al., 2000). Furthermore, GluRIIA was also shown to be required for the PKAdependent modulation of quantal size (Davis et al., 1998).

Glutamate receptor levels can control the number of synapses forming at the NMJ. Favoring GluRIIA over GluRIIB expression results in increased NMJ transmission strength and synapse number (Sigrist et al., 2000; Sigrist et al., 2002; Sigrist et al., 2003). Moreover, applying in vivo imaging of larval NMJ synapses, the formation and growth of individual synapses could be directly correlated with the entry of GluRIIA complexes from diffuse extra-synaptic pools (Rasse et al., 2005). Different from several other postsynaptic proteins tested, GluRIIA shows slow turnover and stably integrates into immature PSDs. 


\subsection{Study design}

Recent reports imply mammalian non-NMDA receptors in the formation and stability of larger postsynaptic subcellular compartments such as dendritic spines independent of their ionic transmission (Kasai et al., 2003; Passafaro et al., 2003). However, whether and how non-NMDA glutamate receptors are involved in the assembly of postsynaptic specializations (PSDs) remained open.

The full absence of non-NMDA glutamate receptors at the Drosophila NMJ results in embryonic lethality due to the incapability of locomotion required for hatching. Nevertheless, extremely reduced levels of muscle glutamate receptors, hardly detectable by immunocytochemistry or electrophysiological recordings, were shown to be sufficient for survival (Marrus et al., 2004; Qin et al., 2005). This allowed studying effects of severe glutamate receptor deprivation on postsynaptic assembly and maturation. While the initial assembly of synapses persisted, the maturation of PSDs specifically failed. During synaptic growth glutamate receptor incorporation into the postsynaptic membrane is likely critical to enlarge PSDs by organizing cell adhesion to bring pre- and postsynaptic membranes in apposition.

The development of the larval neuromuscular system is characterized by a more than 100-fold increase in muscle surface (Jan and Jan, 1976) that goes along with a concomitant boost of evoked junctional currents (EJC), indicating a vast increase in synapse number and synaptic strength (Broadie and Bate, 1993; Schuster et al., 1996; Sigrist et al., 2003). To get an insight in the specific relevance of the two receptor complex subtypes for PSD maturation, single identified PSDs, expressing both fluorescently tagged GluRIIA- and GluRIIB-type complexes, were followed applying in vivo imaging of developing larval NMJs (Rasse et al., 2005). The strong dynamics of the NMJ were reflected in a massive growth of the PSD population caused by specific recruitment of either GluRIIA or GluRIIB containing complexes, which could be linked to the initial subunit composition and size of the PSD. Thereby, the incorporation behavior of the two receptor subtypes, which was analyzed by fluorescence recovery after photobleaching (FRAP) experiments, differed fundamentally. This differential recruitment, which persisted after suppression of presynaptic activity, seems to be based on the differing physiological channel properties and might be regulated by $\mathrm{C}$-terminal receptor phosphorylation. 


\section{Material and Methods}

\subsection{Molecular biology}

\subsubsection{Material}

The following plasmids were used for molecular cloning:

- pBNJ24.6 (generous gift of D. Sheridan, Yale University Medical School, New Haven, USA) (Sheridan et al., 2002)

- pBluescript $^{\circledR}$ II KS + (pKS+; Stratagene, La Jolla, USA)

- pEGFP N1 (Clontech, Palo Alto, USA)

- pSL1180 (Fig. 56; Amersham Pharmacia Biotech, Buckinghamshire, England)

- pSL fa1180fa (Horn and Wimmer, 2000)

- $\operatorname{pUAST}$ (Fig. 57) (Brand and Perrimon, 1993)

- $\quad$ pUAST XL+ (produced in the lab, see 7.1)

All chemicals were, if not stated elsewhere, purchased from Roth (Karlsruhe, Germany), Sigma (St. Louis, USA) or Merck (Darmstadt, Germany). Agarose was obtained from Peqlab (Erlangen, Germany).

Alkaline phosphatase, T4 DNA ligase, T4 polynucleotide kinase, Taq Polymerase and various restriction endonucleases were purchased from Roche (Mannheim, Germany). The restriction endonuclease $A s c l$ as well as Vent DNA-Polymerase was obtained from New England Biolabs (Beverly, USA). Elongase ${ }^{\circledR}$ enzyme mix used for overlap-extension PCRs was purchased from Invitrogen (Karlsruhe, Germany). All oligonucleotides were synthesized by MWG Biotech (Ebersberg, Germany). Unless stated elsewhere all molecular biology kits for RNA or DNA extraction and purification were obtained from Qiagen (Hilden, Germany). Chemically competent $E$. coli XL1 blue cells were produced in the lab.

All PCRs were performed with the PCR System GeneAmp 9700 (Applied Biosystems, Foster City, USA).

\subsubsection{Transgenes}

The molecular cloning of transgenes was performed, if not stated elsewhere, using standard molecular biology procedures (Sambrook et al., 1989). All constructs were double strand sequenced (MWG Biotech, Ebersberg, Germany or Qiagen, Hilden, Germany). Correct sequences were verified with MacVector ${ }^{\mathrm{TM}}$ (Accelrys, San Diego, USA).

Overlap-extension PCRs (Fig. 16) were led through according to the Elongase ${ }^{\circledR}$ kit protocol (Invitrogen, Karlsruhe, Germany) with $30 \pm 2$ overlapping base pairs in between the two respective DNA templates. 
Mixture 1:

10mM dNTP-Mix

$10 \mu \mathrm{M} \quad$ forward primer

$10 \mu \mathrm{M}$ reverse primer

50 $\quad$ DNA template 1

50 $\quad$ DNA template 2

ad $20 \mu \mathrm{l} \quad \mathrm{H}_{2} \mathrm{O}$

Mixture 2:

$\left.\begin{array}{ll}5 \mathrm{x} & \text { buffer A } \\ 5 \mathrm{x} & \text { buffer B }\end{array}\right\} 60 \mathrm{mM}$ Tris-SO $(\mathrm{pH} 9,1), 18 \mathrm{mM}\left(\mathrm{NH}_{4}\right)_{2} \mathrm{SO}_{4}, 1-2 \mathrm{mM} \mathrm{MgSO}_{4}$

$2 \mu \mathrm{l} \quad$ Elongase ${ }^{2}$ enzyme mix

$\operatorname{ad} 30 \mu \mathrm{l} \quad \mathrm{H}_{2} \mathrm{O}$

The mixtures 1 and 2 were combined and the PCR was performed as follows:

$30 x$

$\begin{array}{lll}\text { Denaturation } & 30^{\prime \prime} & 94^{\circ} \mathrm{C} \\ \text { Annealing } & 30^{\prime \prime} & 54^{\circ} \mathrm{C} \\ \text { Extension } & 1^{\prime} \text { per kbp } & 68^{\circ} \mathrm{C}\end{array}$

Step 1: Produce single PCR fragments A, B and C

Step 2: Produce fragment $A B$

$5^{4}$ B

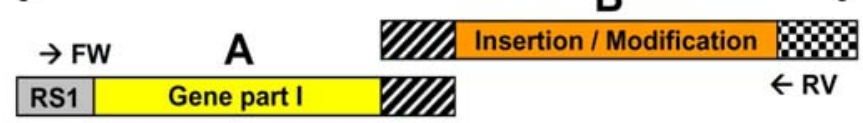

Step 3: Produce fragment ABC

5

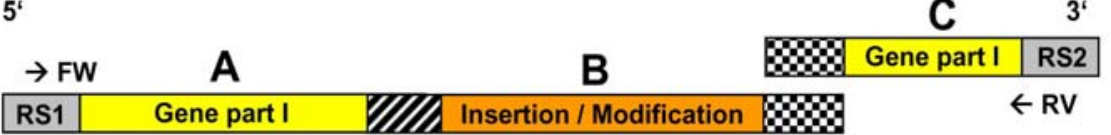

Step 4: Restriction

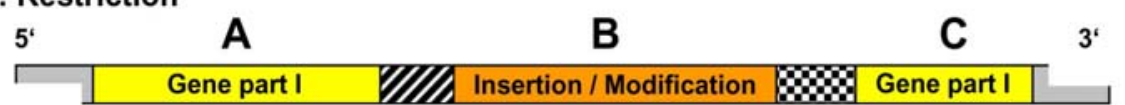

Fig. 16. Overlap extension PCR

Firstly, three single PCR fragments with $30 \pm 2$ overlapping base pairs (striped and checkered regions) are produced using Vent DNA-polymerase. Secondly, the resulting PCR fragments A and $\mathrm{B}$ are combined and subjected to Elongase ${ }^{\circledR}$ overlap-extension PCR. The enzyme mix, which contains Taq polymerase and the Pyrococcus species GB-D polymerase with 3'-5' exonuclease activity, ensures both the fill-up reaction and the rapid template amplification. Thirdly, a PCR of the obtained fragment $A B$ together fragment $C$ leads to the designated full length PCR product. Fourthly, the PCR product ABC is enzymatically restricted at the 5' and 3' end (RS1 and RS2) to enable further cloning steps. 


\subsubsection{Site-directed fluorescence-tagging of gluRIIA and gluRIIB}

gluRIIA $A^{\text {GFP893 }}$ and gluRIIA mRFP893: $^{\text {mas }}$

The cloning of gluRIIA $A^{G F P 893}$ and gluRIIA $A^{\text {mRFP893 }}$ was described recently (Rasse et al., 2005).

\section{gluRIIB, gluR IIB ${ }^{\text {GFP897 }}$ and gluR/IB ${ }^{\text {mRFP897 }}$ (Fig. 17):}

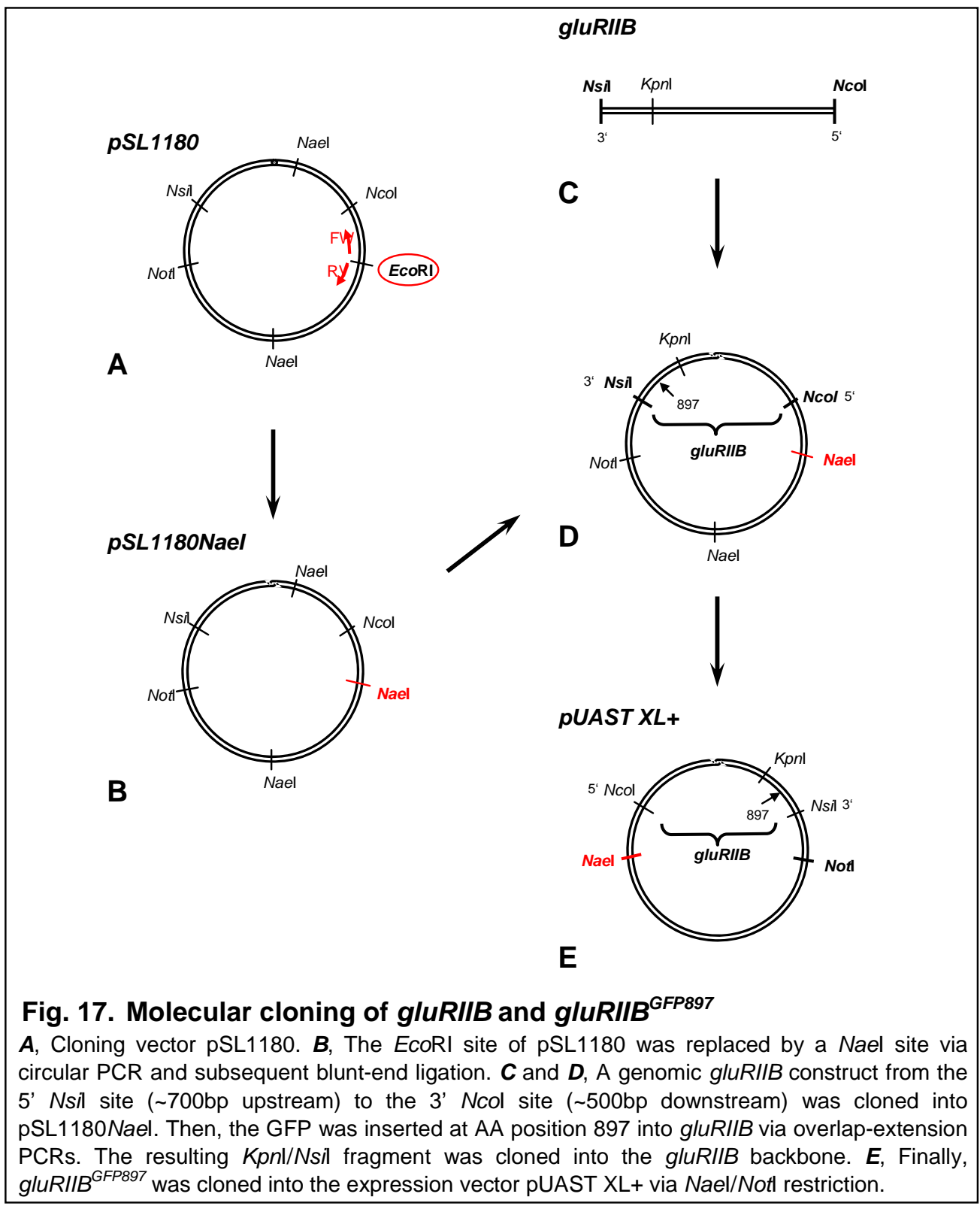

Previously, a genomic HindIII/Sall fragment, covering the whole open reading frame of gluRIIB and the 3' end of gluRIIA was used as genomic gluRIIB rescue construct (DiAntonio et al., 1999). In this study, the gluRIIA 3' end, which is potentially translatable, was cleaved off leaving a genomic Nsil/Ncol construct of gluRIIB (gluRIIB coding region plus $\sim 700 \mathrm{bp}$ upstream and $\sim 500 \mathrm{bp}$ downstream) with unaltered rescue capability. 


\section{pSL1180Nael:}

Cloning of pSL1180Nael (Fig. 17B):

- Circular PCR and blunt-end ligation

o template: pSL1180 (Fig. 17A, Fig. 56)

o forward primer: 5' CCGGCGTAATCATGGTCATAGCTGTTTCCTG 3'

o reverse primer: 5' CGAATGGCCATGGGACGTC 3'

gluRIIB:

Cloning of pSL1180Nael gluRIIB (Fig. 17C):

- Restriction: Nsil/Ncol

o vector: pSL1180Nael

o insert: gluRIIB

Cloning of PUAST XL+ gluRIIB:

- Restriction: Notl/Nael

o vector: $\mathrm{PUAST} \mathrm{XL}+$

o insert: gluRIIB

gluR/IB ${ }^{\text {GFP897: }}$

The fluorophore insertion site and the linker sequences were chosen and designed as recently described (Sheridan et al., 2002; Rasse et al., 2005). Details are depicted in Fig. 32. The linker sequence is shown below in Fig. 18.

Cloning of pSL1180Nael gluRIIB ${ }^{\text {GFP897 }}$ (Fig. 17D):

- Overlap extension PCR, 3 steps (Fig. 16 and 3.1.2)

- Fragment A:

o template: pSL1180Nael gluRIIB

o forward primer: 5' GCAAGGGTACCTATGCCTTCC 3'

o reverse primer: 5' GGCGCGCCAGATGTGTATAAGAGACAT

GTAATTTGCTCCAGCGATGAGTAAC 3'

- Fragment B:

o template: pEGFP N-1

o forward primer: 5' CTCTTATACACATCTGGCGCGCCGA GCAAGGGCGAGGAGCTGT 3'

o reverse primer: 5' TCTGTCTCTTATACACATCTGCCCGGGCGCG CCGCCCTTGTACAGCTCGTCCATGCC 3'

- Fragment C:

o template: pSL1180Nael gluRIIB 
o forward primer: 5' GGCGGCGCGCCCGGGCAGATGTGTATAAGA GACAGAATTACAAGTGCTTCCAGTGCGAAAA 3'

o reverse primer: 5' CCAGCATGCATTTTGCATACA 3'

- Fragment $A B$

$O$ primer: fragment $A$ forward and $B$ reverse

- Fragment $A B C$

o primer: fragment $A$ forward and $C$ reverse

- Restriction: Kpnl/Nsil

o vector: pSL1180Nael gluRIIB

0 insert: PCR product $A B C$

Cloning of pUAST XL+ gluR/IB ${ }^{\text {GFP897 }}$ (Fig. 17E):

- Restriction: Notl/Nael

o vector: $\mathrm{PUAST} X \mathrm{XL}+$

o insert: gluR/IB ${ }^{G F P 897}$

gluR/IB ${ }^{\text {mRFP897: }}$

Cloning of pUAST XL+ gluR/IB ${ }^{m R F P 897}$ :

- Restriction: Ascl

o vector: pUAST XL+ gluR/IB ${ }^{\text {GFP897 }}$ (cleavage of GFP)

o insert: mRFP1 with flanking Ascl sites (Rasse et al., 2005)

\subsubsection{Chimeric glutamate receptor subunits}

AAB (gluR\|A ${ }^{\text {IIB C-term) }}$ :

Cloning of $\mathrm{pSL}$ fa1180fa $A A B$ :

- circular PCR of vector and blunt-end ligation with insert

- vector:

o template: pSL fa1180fa gluRIIA

o forward primer: 5' TAGGTGGTCGGAATATTGGAC 3'

o reverse primer: 5' CCAGGATAACGCCGATGAGAAC 3'

- insert:

o template: $\mathrm{pKS}+$ gluRIIBCDNA

o forward primer: 5' GCATCGCCGAGTTTTTGTGG 3'

o reverse primer: 5' CTACTTTTCAATTCGCCTGGTCT 3'

Cloning of pUAST $A A B$ :

- Restriction: EcoRI/Xhol

o vector: $\mathrm{pUAST}$

0 insert: $A A B$ 
$\underline{A A B^{G F P}}:$

Cloning of $\mathrm{pSL}$ fa1180fa $A A B^{G F P}$ :

- Overlap-extension PCR, 3 steps (Fig. 16 and 3.1.2)

- Fragment A:

o template: pSLfa1180fa gluRIIA

o forward primer: 5' GGGATTAGGTTAACGCTCTTGAGTG 3'

o reverse primer: 5' CCACAAAAACTCGGCGAT GCCCAGGATAACGCCG 3'

- Fragment B:

o template: pSL1180Nael gluRIIB ${ }^{\text {GFP897 }}$

o forward primer: 5' GTTATCCTGGGCATCGCCGAGTTTTTGT GGCACG 3'

o reverse primer: 5' CAATATTCCGACCACCTACTTTTCAATTCGC CTGGTCTT 3'

- Fragment C:

o template: pSL fa1180fa gluRIIA

o forward primer: 5' CGAATTGAAAAGTAGGTGGTCGGAATATTG GACGATTG 3'

o reverse primer: 5' CTCGCTCGAGTGCCGCACTAAAGG 3'

- Fragment $A B$

0 primer: fragment $A$ forward and $B$ reverse

- Fragment $A B C$

0 primer: fragment $A$ forward and $C$ reverse

- Restriction: Hpal/Xhol

o vector: $p S L$ fa1180fa gluRIIA

o insert: PCR product $A B C$

Cloning of pUAST $A A B^{G F P \text { : }}$

- Restriction: EcoRI/Xhol

o vector: pUAST

0 insert: $A A B^{G F P}$

AAC (gluRIIA IIC C-term):

Cloning of pSL fa1180fa AAC:

- circular PCR of vector and blunt-end ligation with insert

- vector:

o template: pSL fa1180fa gluRIIA

o forward primer: 5' TAGGTGGTCGGAATATTGGAC 3' 
o reverse primer: 5' CCAGGATAACGCCGATGAGAAC 3'

- insert:

o template: pSL fa1180fa gluRIICCDNA

o forward primer: 5' GCATCACGGAGTTTTTGGTCTACG 3'

o reverse primer: 5' CTAGACCCTTGCCTCCTTTTCTCC 3'

Cloning of pUAST AAC:

- Restriction: EcoRI/Xhol

o vector: pUAST

0 insert: $A A C$

AAD (gluRIIA ${ }^{\text {IID C-term) }}$

Cloning of pSL fa1180fa $A A D$ :

- Overlap-extension PCR, 3 steps (Fig. 16 and 3.1.2)

- Fragment A:

o template: pSL fa1180fa gluRIIA

o forward primer: 5' CCGCCATTCCAGGATCCAGATG 3'

o reverse primer: 5' GTAGCACCAACATAGGATGCCCAGG

ATAACGCCG 3'

- Fragment B:

o template: $p K S+$ gluRIIDcDNA

o forward primer: 5' GTTATCCTGGGCATCCTATGTTGGTGCTA

CTTTGTCTACAAG 3'

o reverse primer: 5' CAATATTCCGACCACTTAATCCTCAAC CGGCATATTTTC 3'

- Fragment C:

o template: pSL fa1180fa gluRIIA

o forward primer: 5' CCGGTTGAGGATTAAGTGGTCGGA

ATATTGGACGATTG 3'

o reverse primer: 5' TACCCAAATGCGCTATCTGTGTTCT 3'

- Fragment $A B$

o primer: fragment $A$ forward and $B$ reverse

- Fragment $A B C$

0 primer: fragment $A$ forward and $C$ reverse

- Restriction: BamHI/Ncol

o vector: $\mathrm{pSL}$ fa1180fa gluRIIA

o insert: PCR product $A B C$ 
Cloning of pUAST AAD:

- Restriction: Nael/Xhol

o vector: pUAST gluRIIA GFP893

0 insert: $A A D$

AAE (gluRIIA IIE C-term):

Cloning of pSL fa1180fa $A A E$ :

- Overlap-extension PCR, 3 steps (Fig. 16 and 3.1.2)

- Fragment A:

o template: pSL fa1180fa gluRIIA

o forward primer: 5' CCGCCATTCCAGGATCCAGATG 3'

o reverse primer: 5' CAAAACCCAGCTGATGATGCCCAG

GATAACGCCG 3'

- Fragment B:

o template: $\mathrm{pKS}+$ gluRIIEcDNA

o forward primer: 5' GTTATCCTGGGCATCATCAGCTGGGT

TTTGTTCGTAATG 3'

o reverse primer: 5' CAATATTCCGACCACCTACTGCGATTCCTGGGCC 3'

- Fragment C:

o template: pSL fa1180fa gluRIIA

o forward primer: 5' CAGGAATCGCAGTAGGTGGTCGGA

ATATTGGACGATTG 3'

o reverse primer: 5' TACCCAAATGCGCTATCTGTGTTCT 3'

- Fragment $A B$

0 primer: fragment $A$ forward and $B$ reverse

- Fragment $A B C$

o Primer: fragment $A$ forward and $C$ reverse

- Restriction: BamHI/Ncol

o vector: $\mathrm{pSL}$ fa1180fa gluRIIA

0 insert: PCR product $A B C$

Cloning of pUAST AAE:

- Restriction: EcoRI/Xhol

o vector: PUAST

0 insert: $A A E$ 
BBA (gluR/IB ${ }^{\| A}$ C-term) $:$

Cloning of pUAST BBA:

- Overlap-extension PCR, 2 steps (Fig. 16 and 3.1.2)

- Fragment A:

o template: $p K S+$ gluRIIBCDNA

o forward primer: 5' GAGCAGATGTATGCACGGTGTACAGTTCCTGG 3'

o reverse primer: 5' CCACAGGAACTCAAAGACACCGATCACGAGTCC 3'

- Fragment B:

o template: $\mathrm{pKS}+$ gluRIIAcDNA

o forward primer: 5' CTCGTGATCGGTGTCTTTGAGTTCCTGTGGAACG 3'

o reverse primer: 5' GCAGTCTAGACTAGCTAACCGTCTTGCTGCG 3'

- Fragment $A B$

0 primer: fragment $A$ forward and $B$ reverse

- Restriction: Bg/ll/Xbal

o vector: PUAST

0 insert: PCR product $A B$

$B B A^{G F P}:$

Cloning of pSL1180Nael BBA ${ }^{\text {GFP }}$ :

- Overlap extension PCR, 3 steps (Fig. 16 and 3.1.2)

- Fragment A:

o template: pSL1180Nael gluRIIB

o forward primer: 5' GCAAGGGTACCTATGCCTTCC 3'

o reverse primer: 5' CCACAGGAACTCAAAGACACCGATCACGAGTCC 3'

- Fragment B:

o template: pSL fa1180fa gluRIIA ${ }^{\text {GFP893 }}$

o forward primer: 5' CTCGTGATCGGTGTCTTTGAGTTCCTGTGGAACG 3'

o reverse primer: 5' AGCCTTTTTACTCCCCTAGCTAACCGT

\section{CTTGCTGCG 3'}

- Fragment C:

o template: pSL1180Nael gluRIIB

o forward primer: 5' CAAGACGGTTAGCTAGGGGAGTAAAAAGG CTTGCGAAC 3'

o reverse primer: 5' CCAGCATGCATTTTGCATACA 3'

- Fragment AB

o primer: fragment $A$ forward and $B$ reverse 
- Fragment $A B C$

0 primer: fragment $A$ forward and $C$ reverse

- Restriction: Kpnl/Nsil

o vector: pSL1180Nael gluRIIB

o insert: PCR product $A B C$

Cloning of pUAST $\mathrm{XL}+B B A^{G F P}$ :

- Restriction: Notl/Nael

o vector: $\mathrm{PUAST} \mathrm{XL}+$

0 insert: $B B A^{G F P}$

CCA (gluR\|l ${ }^{\| A}$ C-term)

Cloning of pUAST CCA:

- Overlap-extension PCR, 2 steps (Fig. 16 and 3.1.2)

- Fragment A:

o template: pKS+ gluRIICCDNA

o forward primer: 5' GAGCAGATCTATGAAAAAAGAACTGTCCGGAAAT 3'

o reverse primer: 5' CCACAGGAACTCAAAAATGCCGATG

AGAAAGGCAATC 3'

- Fragment B:

o template: pSL fa1180fa gluRIIA

o forward primer: 5' TTTCTCATCGGCATTTTTGAGTTCCTGTGGAACG 3'

o reverse primer: 5' GCAGTCTAGACTAGCTAACCGTCTTGCTGCG 3'

- Fragment $A B$

0 primer: fragment $A$ forward and $B$ reverse

- Restriction: Bg/lI/Xbal

o vector: pUAST

0 insert: PCR product $A B$

\subsubsection{Modifications of gluRIIA}

gluRIIA hypo:

The cloning of gluRIIA hypo, a genomic gluRIIA construct missing most of the 3' untranslated region (UTR), was described recently (Qin et al., 2005).

gluRIIA Q614R: $^{2}$

Cloning of $\mathrm{pSL}$ fa1180fa gluRIIA $\mathrm{Q}^{\mathrm{Q614R} \text { : }}$

- Overlap extension PCR, 2 steps (Fig. 16 and 3.1.2) 
- Fragment A:

o template: pSL fa1180fa gluRIIA

o forward primer: 5' GCAGCGCATCCACTTCAACCT 3'

o reverse primer: 5' CTAGGCAGAATGTCGCAGCCCTGTCTCATAA TGGAGCCCACCATCAGCCAAG 3'

- Fragment B:

o template: pSL fa1180fa gluRIIA

o forward primer: 5' CTTGGCTGATGGTGGGCTCCATTATGAGACA GGGCTGCGACATTCTGCCTAG 3'

o reverse primer: 5' TACCCAAATGCGCTATCTGTGTTCT 3'

- Fragment AB

0 primer: fragment $A$ forward and $B$ reverse

- Restriction: Bglll/Ncol

o vector: $\mathrm{pSL}$ fa1180fa gluRIIA

0 insert: $P C R$ product $A B$ Cloning of pUAST gluRIIA $A^{\mathrm{Q} 614 R}$ :

- Restriction: EcoRI/Xhol

o vector: pUAST

o insert: gluRIIA $A^{Q 614 R}$

gluRIIA ${ }^{E 783 A}$ :

Cloning of $\mathrm{pSL}$ fa1180fa gluRIIA ${ }^{E 783 A}$ :

- Overlap extension PCR, 2 steps (Fig. 16 and 3.1.2)

- Fragment A:

o template: $\mathrm{pSL}$ fa1180fa gluRIIA

o forward primer: 5' GCAGCGCATCCACTTCAACCT 3'

o reverse primer: 5' CATCTTCTGCAGCTCGCCCCTGGCGCTCAG CTGGAGAATGGACACG 3'

- Fragment B:

o template: pSL fa1180fa gluRIIA

o forward primer: 5' CGTGTCCATTCTCCAGCTGAGCGCCAGGG GCGAGCTGCAGAAGATG 3'

o reverse primer: 5' TACCCAAATGCGCTATCTGTGTTCT 3'

- Fragment AB

0 primer: fragment $A$ forward and $B$ reverse

- Restriction: Bglll/Ncol

o vector: $\mathrm{pSL}$ fa1180fa gluR/IA 
0 insert: PCR product $A B$

Cloning of pUAST gluRIIA ${ }^{E 783 A}$ :

- Restriction: EcoRI/Xhol

o vector: PUAST

o insert: gluRIIA

gluR $\| A^{\Delta C 17}$ :

Cloning of pUAST gluRIIA ${ }^{\Delta C 17}$ :

- circular PCR and blunt-end ligation

o template: pSL fa1180fa gluRIIA

o forward primer: 5' TAGGTGGTCGGAATATTGGACG 3'

o reverse primer: 5' TGATCGCCTGGACGACGACTTG 3'

- restriction: EcoRI/Xhol

o vector: pUAST

o insert: gluRIIA $A^{\Delta C 17}$

gluRIIA ${ }^{\Delta C 35}$ :

Cloning of pUAST gluRIIA ${ }^{\Delta C 35}$ :

- circular PCR and blunt-end ligation

o template: pSL fa1180fa gluRIIA

o forward primer: 5' TAGGTGGTCGGAATATTGGACG 3'

o reverse primer: 5' GCGCACCCAAAACTTCAGG 3'

- restriction: EcoRI/Xhol

o vector: PUAST

o insert: gluR/IA $A^{\Delta C 35}$

gluRIIA ${ }^{\Delta C 44}$ :

Cloning of pUAST gluRIIA ${ }^{\Delta C 44}$ :

- circular PCR and blunt-end ligation

o template: pSL fa1180fa gluRIIA

o forward primer: 5' TAGGTGGTCGGAATATTGGACG 3'

o reverse primer: 5' GAGCTCCGCCTTGAAAGCC 3'

- restriction: EcoRI/Xhol

o vector: PUAST

o insert: gluRIIA $A^{\Delta C 44}$ 
gluRIIA ${ }^{\Delta C 53}$ :

Cloning of pUAST gluRIIA ${ }^{\Delta C 53}$ :

- circular PCR and blunt-end ligation

o template: pSL fa1180fa gluRIIA

o forward primer: 5' TAGGTGGTCGGAATATTGGACG 3'

o reverse primer: 5' AGTCACTCGCTCCTCCACCG 3'

- restriction: EcoRI/Xhol

o vector: pUAST

0 insert: gluR/IA $A^{\Delta C 53}$

\subsubsection{In vitro transposition}

(in collaboration with Anne Grünewald)

The in vitro transposition (IVT; Fig. 18, scheme for pSL1180Nael gluRIIB) was performed with the EZ::TN ${ }^{\mathrm{TM}}$ Transposase Kit of Epicentre (Madison, USA). To start, the transposon $T g P T-0$ was amplified from pBNJ24.6 by Vent-polymerase PCR from its mosaic ends with the TN5 ME primer (5' CTGTCTCTTATACACATCT 3'). Then, the following reagents were mixed:

$\begin{array}{ll}1 \mu \mathrm{l} & \text { 10x reaction buffer } \\ 0,2 \mu \mathrm{g} & \text { target DNA (pSLfa1180fa gluRIIA or pSL1180Nael gluRIIB) } \\ \mathrm{X} \mu \mathrm{l} & \text { transposon TgPT-O (molar equivalent to target DNA) } \\ 1 \mu \mathrm{l} & \text { Transposase } \\ \text { ad 10 } \mathrm{Ll} & \mathrm{H}_{2} \mathrm{O}\end{array}$

After incubation for $2 \mathrm{~h}$ at $37^{\circ} \mathrm{C}$ the reaction was stopped with the provided $10 \mathrm{x}$ stop solution $\mathrm{EZ}: \mathrm{TN}^{\mathrm{TM}}$ and the mixture was transformed into chemically competent $E$. coli XL1blue cells. Positive clones, containing both kanamycin and ampicillin resistance genes, were identified for insertions of TgPT-O within the target DNA via colony PCR using the following primer pairs:

$$
\begin{array}{ll}
\text { forward primer } & \text { 5' GCTGAGATGACATTCTTGGC 3' (gluR IIA) } \\
& \text { 5' CATTCACTGGTCCATTTCC 3' (gluR IIB) }
\end{array}
$$

5'3' insertion: reverse primer 5' AACAGCTCCTCGCCCTTG 3' (within GFP)

3'5' insertion: reverse primer 5' CCTGCCATCACGAGATTT 3' (within KanaR)

Positive samples harboring $T g P T-0$ within the translated region of the target gene were sequenced to identify in frame insertions and the exact position.

Clones with 3'5' insertion were subjected to enzymatic Ascl digest and re-ligation to change the EGFP orientation (gluRIIA, which contains an internal Ascl site, had to be subcloned via Sall/Ncol restriction). Positive clones were again identified via 
colony PCR. Finally, the kanamycin resistance was eliminated from all obtained clones by Srfl restriction and all constructs were cloned into pUAST (gluRIIA, via EcoRI/Xhol) or pUAST XL+ (gluRIIB, via Notl/Nael).

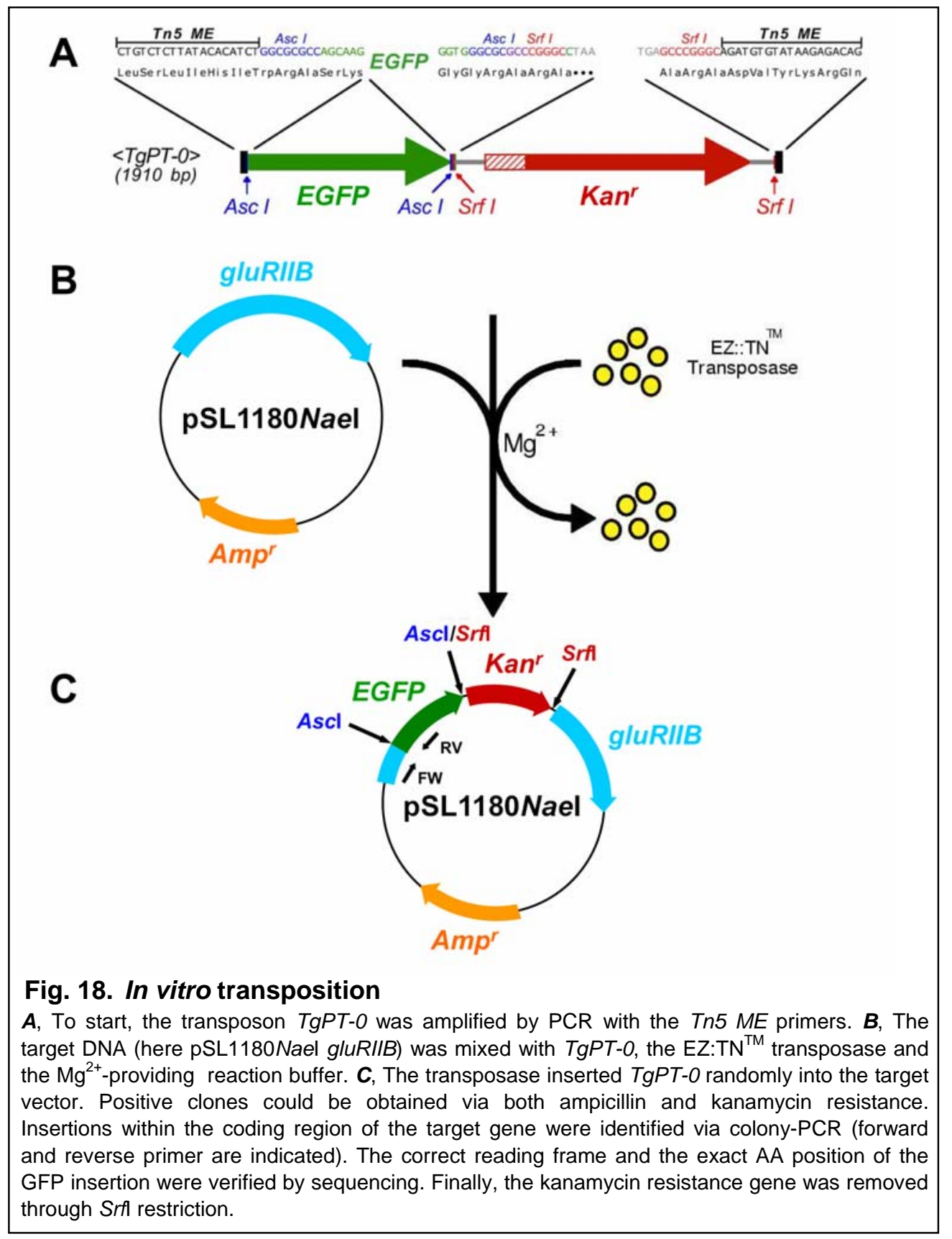

\subsection{Drosophila melanogaster}

\subsubsection{Cultivation}

Fly strains were, if not otherwise stated, reared at $25^{\circ} \mathrm{C}$ in plastic bottles (Greiner

Bio-one, Kremsmünster, Austria) containing cultivation medium (195g agar, 200g soy flour, $360 \mathrm{~g}$ yeast, $1600 \mathrm{~g}$ corn flour, $440 \mathrm{~g}$ beet syrup, $1600 \mathrm{~g}$ malt, $30 \mathrm{~g}$ nipagine, 
$126 \mathrm{ml}$ propionic acid, ad $18 \mathrm{I} \mathrm{H}_{2} \mathrm{O}$ ). Embryonic collections were performed in plastic cylinders placed on apple agar plates (1l apple juice, $100 \mathrm{~g}$ saccharose, $85 \mathrm{~g}$ agaragar, 40ml nipagine (15\%), ad $3 \mathrm{l} \mathrm{H}_{2} \mathrm{O}$ ).

\subsubsection{Transgenics}

Drosophila germ line transformation was performed with an Eppendorf InjectMan (Hamburg, Germany) as described previously (Rubin and Spradling, 1982) using 300ng/ $\mu \mathrm{l}$ P-element DNA (pUAST with inserted transgene) and 100ng/ $\mu$ l helper plasmid ( $\mathrm{p} \Delta 2-3)$. Transgenic animals were established in the following genetic backgrounds:

\begin{tabular}{|c|c|c|}
\hline Name & Genetics & Source \\
\hline$w 1$ & $\frac{w-}{w-} ; \frac{+}{+} ; \frac{+}{+}$ & (Castiglioni, 1951) \\
\hline$d f c / h^{4}$ & $\frac{w-}{w-} ; \frac{d f(2 L) c l^{h 4}}{G l a, B c} ; \frac{+}{+}$ & (Petersen et al., 1997) \\
\hline$d f a s t^{4}$ & $\frac{w-}{w-} ; \frac{d f(2 L) a s t^{4}}{G l a, B c} ; \frac{+}{+}$ & (Marrus et al., 2004) \\
\hline E3 & $\frac{w-}{w-} ; \frac{+}{+} ; \frac{E 3}{T M 6 B}$ & (Qin et al., 2005) \\
\hline
\end{tabular}

Table 1. Fly stocks for germ line transformation

$d f c / h^{4}$ is a deficiency for gluRIIA and gluRIIB, $d f a s t^{4}$ for gluRIIC and E3 for gluRIID and gluRIIE.

\subsubsection{Genetics}

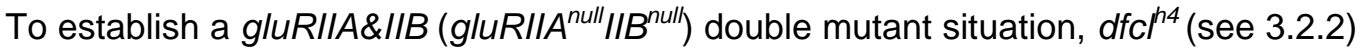
had to be crossed to $d f(2 L) g l u R I I A \& B^{S P 22}$ (from here on referred to as $A 22$ ) (Petersen et al., 1997; DiAntonio et al., 1999; Qin et al., 2005). gluRIIC (gluRIII) mutants were produced by crossing gluRIII ${ }^{1}$ to $d f(2 L) a s t^{4}$ or $d f(2 L) a s t^{2}$ (Marrus et al., 2004). Leaky expression of GluRIIC (IIC hypo ) was performed as previously described (Marrus and DiAntonio, 2004). As wild type control $w^{1}$ (see Table 1) was used. Rescue embryos and larvae were selected using marked balancer chromosomes (either Bc (black cell) or GFP).

The genetics of all used animals are summarized in Table 2. All transgenes (see Fig. 62, Fig. 63, Fig. 64) derived, if not stated elsewhere, from genomic constructs. In cases were the gender was genetically of no importance, females and males were chosen stochastically. 


\begin{tabular}{|c|c|}
\hline Name & Genetics \\
\hline$\left\|A^{\text {null }}\right\| B^{\text {null }}$ & $\frac{w-}{w-} ; \frac{d f c l^{h 4}}{A 22} ; \frac{+}{+}$ \\
\hline$\| C^{\text {null }}$ & $\frac{w-}{w-} ; \frac{\text { dfast }^{4}}{\text { III }^{1}} ; \frac{+}{+}$ \\
\hline$I I A$ & $\frac{w-}{w-} ; \frac{d f c l^{h 4}}{A 22} ; \frac{U A S-g l u R I I A}{+}$ \\
\hline$I I A^{\text {hypo }}$ & $\frac{w-}{w-} ; \frac{d f c l^{h 4}, U A S-g^{\prime} \text { luRIIA }}{\text { hypo }} ; \frac{+}{+}$ \\
\hline$I I A^{\text {hypo }}+$ elav-tnt & $\frac{w-}{w-} ; \frac{d f c l^{h 4}, U A S-g_{l u R I I A}{ }^{\text {hypo }}}{A 22, U A S-t n t} ; \frac{\text { elav - gal } 4}{+}$ \\
\hline$I / C^{\text {hуро }}$ & 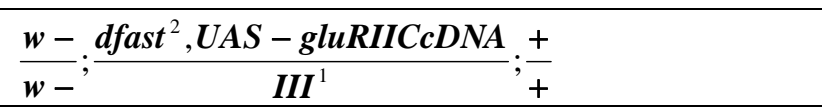 \\
\hline$I I A^{\Delta C 17}$ & $\frac{U A S-g l u R I I A^{\Delta C 17}}{w-} ; \frac{d f c l^{h 4}}{A 22} ; \frac{+}{+}$ \\
\hline$I I A^{\Delta C 35}$ & $\frac{w-}{w-} ; \frac{d f l^{h 4}}{A 22} ; \frac{U A S-g_{l u R I I A}{ }^{\Delta C 35}}{+}$ \\
\hline$\| A^{\Delta C 44}$ & $\frac{w-}{w-} ; \frac{d f c l^{h 4}, U A S-\text { gluRIIA }^{\Delta C 44}}{A 22} ; \frac{+}{+}$ \\
\hline $11 A^{\Delta C 53}$ & $\frac{w-}{w-} ; \frac{d f c l^{h 4}, U A S-\text { gluRIIA }^{\Delta C 53}}{A 22} ;+$ \\
\hline$\| A^{Q 614 R}$ & $\frac{w-}{w-} ; \frac{d f c l^{h 4}}{A 22} ; \frac{U A S-g l u R I I A^{Q 614 R}}{+}$ \\
\hline$I I A^{E 783 A}$ & $\frac{w-}{w-} ; \frac{d f c l^{h 4}, U A S-g l u R I I A^{E 783 A}}{A 22} ; \frac{+}{+}$ \\
\hline$\| A^{G F P}$ & $\frac{w-}{w-} ; \frac{d f c l^{h 4}}{A 22} ; \frac{U A S-g l u R I I A^{G F P 893}}{+}$ \\
\hline $2 x \| A^{G F P}$ & $\frac{w-}{w-} ; \frac{d f c h^{h 4}}{A 22} ; \frac{U A S-g^{\prime} \text { URIIIA } A^{\text {GFP893 }}}{\text { UAluRIIA }}$ \\
\hline$\left\|A^{G F P} \&\right\| A^{m R F P}$ & $\frac{w-}{w-} ; \frac{d f c l^{h 4}}{A 22} ; \frac{U A S-g l u R I I A^{G F P 893}}{U A S-g l u R I I A^{m R F P 893}}$ \\
\hline $\begin{array}{l}I I A^{G F P} \& \| A^{m R F P} \\
+ \text { ok319-tnt }\end{array}$ & $\frac{w-}{w-} ; \frac{d f c l^{\text {h4 }}, \text { ok } 319-\text { gal } 4}{A 22, \text { UAS }- \text { tnt }} ; \frac{\text { UAS }- \text { gluRIIA }^{\text {GFP } 893}}{\text { UAS }- \text { gluRIIA }}$ \\
\hline$I I B$ & $\frac{w-}{w-} ; \frac{d f c l^{h 4}}{A 22} ; \frac{U A S-g l u R I I B}{+}$ \\
\hline$I I B^{G F P}$ & $\frac{w-}{w-} ; \frac{d f c l^{h 4}}{A 22} ; \frac{U A S-g l u R I I B^{G F P 897}}{+}$ \\
\hline $2 x \| B^{G F P}$ & $\frac{w-}{w-} ; \frac{d f c l^{h 4}, U A S-g l u R I I B^{G F P 897}}{A 22} ; \frac{U A S-g l u R I I B^{G F P 897}}{+}$ \\
\hline$\left\|B^{G F P} \&\right\| B^{m R F P}$ & $\frac{w-}{w-} ; \frac{d f c l^{h 4}}{A 22} ; \frac{U A S-g l u R I I B^{G F P 897}}{U A S-g l u R I I B^{m R F P 897}}$ \\
\hline $\begin{array}{l}I I B^{G F P} \& / I B^{m R F P} \\
+ \text { ok319-tnt }\end{array}$ & $\frac{w-}{w-} ; \frac{d f c l^{h 4}, o k 319-g a l 4}{A 22, U A S-\text { tht }} ; \frac{U A S-\text { gluRIIB }^{\text {GFP } 897}}{U A S-\text { gluRIIA }^{\text {mRFP } 897}}$ \\
\hline$\left\|B^{G F P} \&\right\| A^{m R F P}$ & 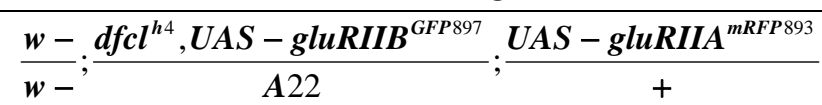 \\
\hline
\end{tabular}




\begin{tabular}{|c|c|}
\hline $\begin{array}{l}I I B^{G F P} \& \| A^{m R F P} \\
+o k 319-t n t\end{array}$ & $\frac{w-}{w-} ; \frac{d f c l^{h 4}, o k 319-g a l 4}{A 22, U A S-\text { tht }} ; \frac{U A S-g l u R I I B^{\text {GFP897 }}}{U A S-g l u R I I A^{\text {mRFP } 893}}$ \\
\hline$\left\|A^{G F P} \&\right\| B^{m R F P}$ & 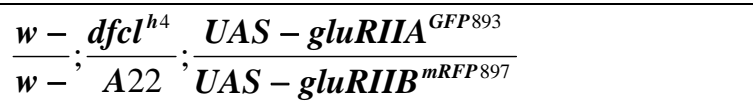 \\
\hline$A A B$ & $\frac{w-}{w-} ; \frac{d f c l^{h 4}}{A 22} ; \frac{U A S-A A B}{+}$ \\
\hline$A A B^{G F P} \& \| A^{m R F P}$ & $\frac{w-}{w-} ; \frac{d f c l^{h 4}, U A S-A A B^{G F P}}{A 22} ; \frac{U A S-g l u R I I A^{m R F P ~} 893}{+}$ \\
\hline$A A B^{G F P} \& \| B^{m R F P}$ & $\frac{w-}{w-} ; \frac{d f c l^{h 4}, U A S-A A B^{G F P}}{A 22} ; \frac{U A S-g l u R I I B^{m R F P 897}}{+}$ \\
\hline$A A C$ & $\frac{w-}{w-} ; \frac{d f l^{h 4}}{A 22} ; \frac{U A S-A A C}{+}$ \\
\hline$A A D$ & $\frac{w-}{w-} ; \frac{d f c l^{h 4}}{A 22} ; \frac{U A S-A A D}{+}$ \\
\hline$A A E$ & $\frac{w-}{w-} ; \frac{d f c l^{h 4}}{A 22} ; \frac{U A S-A A E}{+}$ \\
\hline$B B A$ & $\frac{w-}{w-} ; \frac{d f c l^{h 4}, U A S-B B A c D N A}{A 22} ; \frac{M h c-g a l 4}{+}$ \\
\hline$B B A^{G F P} \& \| A^{m R F P}$ & $\frac{w-}{w-} ; \frac{d f c l^{h 4}, U A S-B B A^{G F P}}{A 22} ; \frac{U A S-g l u R I I A^{m R F P 893}}{+}$ \\
\hline$B B A^{G F P} \& \| B^{m R F P}$ & $\frac{w-}{w-} ; \frac{d f c l^{h 4}, U A S-B B A^{G F P}}{A 22} ; \frac{U A S-g l u R I I B^{m R F P 897}}{+}$ \\
\hline$C C A$ & $\frac{w-}{w-} ; \frac{d f c l^{h 4}}{A 22} ; \frac{U A S-C C A c D N A}{M h c-g a l 4}$ \\
\hline ok319-2XEYFP & $\frac{w-}{w-} ; \frac{o k 319-\text { gal } 4}{+} ; \frac{\text { UAS }-2 x E Y F P}{+}$ \\
\hline ok319-tnt & $\frac{w-}{w-} ; \frac{o k 319-g a l 4}{U A S-t n t} ; \frac{+}{+}$ \\
\hline cha-shi $i^{T S 1}\left(29^{\circ} \mathrm{C}\right)$ & $\frac{U A S-\text { shi }^{T S 1}}{w-} ; \frac{\text { cha }-g a l 4}{+} ; \frac{+}{+}$ \\
\hline elav-tnt & $\frac{w-}{w-} ; \frac{U A S-\operatorname{tnt}}{+} ; \frac{\text { elav }- \text { gal } 4}{+}$ \\
\hline$s h i^{T S 1}\left(32^{\circ} \mathrm{C}\right)$ * & $\frac{\operatorname{shi}^{T S 1}}{\operatorname{shi}^{T S 1}} ; \frac{+}{+} ; \frac{+}{+}$ \\
\hline $\operatorname{para}^{T S}\left(29^{\circ} \mathrm{C}\right)$ & $\frac{\text { para }^{T S}}{\text { para }^{T S}} ; \frac{+}{+} ; \frac{+}{+}$ \\
\hline $\operatorname{cac}^{G F P}$ & $\frac{w-}{w-} ; \frac{o k 6-g a l 4}{+} ; \frac{U A S-c^{G a c}}{+}$ \\
\hline$c a c^{G F P}+\| A^{\text {hypo }}$ & $\frac{w-}{w-} ; \frac{d f c l^{h 4}, U A S-g_{l u R I I A}{ }^{\text {hypo }}}{A 22, o k 6-g a l 4} ; \frac{U A S-\text { cac }^{G F P}}{+}$ \\
\hline
\end{tabular}

Table 2. Genetics

Genetics of all used genotypes. The $4^{\text {th }}$ chromosome is not illustrated. * $s h i^{T S 1}$ embryos were collected for $2 \mathrm{~h}$, aged $12 \mathrm{~h}$ at $25^{\circ} \mathrm{C}$ and then shifted to the restrictive temperature of $32^{\circ} \mathrm{C}$ until dissection. In the text, glutamate receptor genotypes also appear with the prefix gluR. 


\subsubsection{Immunohistochemistry}

For all preparations hemolymph-like (HL-3) saline without $\mathrm{Ca}^{2+}$ (Stewart et al., 1994) was used (concentrations in $\mathrm{mM}$ ): $\mathrm{NaCl} 70, \mathrm{KCl} 5, \mathrm{MgCl}_{2} 20, \mathrm{NaHCO}_{3} 10$, trehalose 5, sucrose 115, HEPES 5, pH adjusted to 7.2.

\subsubsection{Larval body-wall preparation}

$2^{\text {nd }}$ or $3^{\text {rd }}$ instar larvae were fixed on a rubber dissection pad with fine insect pins (0.1x10mm, Thorns, Göttingen, Germany) and covered with a drop of cold HL-3 solution. Then, the larvae were opened dorsally along the midline from the posterior to the anterior end with dissection spring scissors (FST, Vancouver, Canada). Subsequently, all internal organs including the central nervous system were removed carefully with fine forceps (FST, Vancouver, Canada) and the epidermis was stretched and pinned down with two pins on each side.

\subsubsection{Embryonic body-wall preparation}

The embryos (stage 17, 20-22h after egg laying) were washed within a micro sieve and their chorion membrane was removed with 50\% DanChlorix (Colgate-Palmolive, Hamburg, Germany). Next, the embryos were carefully pressed out of their vitellin membrane with fine forceps. For dissection the embryos were fixed with fine clips on a sylgard (Dow Corning, Midland, USA) plate and opened dorsally along the midline using two ultra-thin electrolytically sharpened tungsten needles (tungsten wire, $0.075 \mathrm{~mm}$; World Precision Instruments, Sarasota, USA. Then, internal organs were sucked off and the epidermis was pinned down with a tungsten needle on each side.

\subsubsection{Fixation and staining}

The dissected samples were fixed either for 10' with 4\% paraformaldehyde (PFA) in PBS (8g NaCl, $2 \mathrm{~g} \mathrm{KCl}, 2 \mathrm{~g} \mathrm{KH}_{2} \mathrm{PO}_{4}, 1.15 \mathrm{~g} \mathrm{Na} \mathrm{HPO}_{4} \times 2 \mathrm{H}_{2} \mathrm{O}$, ad $1 \mathrm{l} \mathrm{H}_{2} \mathrm{O}, \mathrm{pH}$ 7.4) or for 5' with ice-cold methanol (for 8B4D2 stainings). After 30' of blocking with PBT (PBS with $0.05 \%$ Triton TX100) containing 5\% goat serum (NGS), the PBT/NGS solution was refreshed, primary antibodies were added and the dissections were incubated over night at $4^{\circ} \mathrm{C}$. The next day the samples were washed twice shortly and three times for 20' with PBT. Then, fluorescence-labeled secondary antibodies (Dianova, Hamburg, Germany) were applied $1: 500$ for $2 \mathrm{~h}$ in PBT with $5 \%$ NGS. The dissections were washed as before and mounted on an object slide in VectaShield Mounting Medium for fluorescence (Vector Laboratories, Burlingame, USA). 
Primary antibodies were used at the following concentrations:

- mouse anti-GluRIIA (8B4D2; Developmental Studies Hybridoma Bank, lowa City, USA), 1:100

- rabbit anti-GluRIIB (gift of A. DiAntonio, Washington University School of Medicine, St. Louis, USA), 1:2000

- rabbit anti-GluRIIC (Qin et al., 2005), 1:500

- rabbit anti-GluRIID (Qin et al., 2005), 1:500

- mouse Nc82 (gift of E. Buchner, University of Würzburg, Würzburg, Germany), $1: 100$

- rabbit anti-a-Adaptin, (gift of M. Gonzalez-Gaitan, MPI of CBG, Dresden, Germany), 1:50

- rabbit anti-PAK (gift of N. Harden, Simon Fraser University, Burnaby, Canada), $1: 2000$

- mouse anti-FasII (1D4; Developmental Studies Hybridoma Bank, Iowa City, USA), 1:50

- mouse anti-Dlg (4F3; Developmental Studies Hybridoma Bank, lowa City, USA), 1:500

- mouse anti-GFP 3E6 (A-11120; Molecular Probes, Eugene, USA), 1:200

- rabbit anti-GFP (A-11122; Molecular Probes, Eugene, USA), 1:500

- goat anti-HRP cyanine 5 (Dianova, Hamburg, Germany), 1:200

To visualize the muscle morphology TRITC-coupled Phalloidine (P1951, Sigma, St. Louis, USA), which binds to Actin, was applied for 30 minutes in 1:200 dilution (together with the secondary antibodies).

\subsection{Microscopy and image analysis}

\subsubsection{Animal sorting}

The sorting of normal adult flies was performed on a self-made $\mathrm{CO}_{2}$ frit using binocular microscopes (Stemi 2000, Zeiss, Jena, Germany) equipped with halogen lamps (KL200, Schott, Mainz, Germany).

GFP-marked embryos, larvae and adults were sorted using a binocular fluorescence microscope (Fluo ${ }^{\mathrm{TM}}$ MZFLIII, Leica Biosystems, Heidelberg, Germany) equipped with a GFP filter.

\subsubsection{In vivo imaging}

In vivo imaging was performed on a Leica DM IRE2 inverted microscope equipped with a Leica TCS SP2 AOBS scan head and a HCX PL Apo CS 63x 1.32 NA OIL UV objective. The following settings were applied: 
- GFP (green fluorescent protein):

o excitation: 488nm (Ar/ArKr laser)

o detection: 495 - 540nm, gain 750V

- $\operatorname{mRFP}$ (monomeric red fluorescent protein):

o excitation: $561 \mathrm{~nm}$ (He/HeNe laser)

o detection: $570-630 \mathrm{~nm}$, gain $800 \mathrm{~V}$

- format: $512 \times 512$ pixel

- pixel size: $98 \times 98 \mathrm{~nm}$

- z-distance: 488nm

- line averaging: 4

- pinhole: 1.5 airy units

All in vivo imaging experiments were done as recently presented (Rasse et al., 2005) (for an illustration of the set-up see Fig. 65). In short, early $3^{\text {rd }}$ instar larvae with a standardized size of $3.0 \mathrm{~mm}$ to $3.5 \mathrm{~mm}$ were selected and mounted inside an airproof anaesthetization chamber between two $0.12 \mathrm{~mm}$ coverslips, which were kept apart by a thin plastic disc with a slit in the centre. The thickness of the disc and the size of the slit were adjusted to the size of the larvae. The lower coverslip was covered with Voltalef H 10 S oil (Lehman \& Voss, Hamburg, Germany) to enable optimal optical access to the ventral larval body wall muscles. Finally, a metal ring was placed onto the upper coverslip to fix the animal position. To anesthetize the larvae a mixture of air and Suprane $^{\circledR}$ containing the agent desfluran (Baxter, Unterschleißheim, Germany) was pumped into the chamber for about 10 to 12 seconds. The anesthetic freezes all internal movement, which is necessary for undisturbed imaging of the NMJ synapses. Recently it was shown, that even several rounds of anaesthetization do not interfere with further growth and function of the synaptic system (Rasse et al., 2005).

To focus on a specific NMJ (usually NMJ 27, in some cases NMJ 14), halogen light was used to identify the respective muscle. All images were taken from the abdominal segments A2-6. For FRAP (fluorescence recovery after photobleaching) experiments either the mRFP (561nm laser) or both the GFP and mRFP channel (488nm laser) were bleached until residual fluorescent signals were no longer detectable in the respective $\mathrm{NMJ}$ part.

After each imaging session (maximally 30 minutes) single larvae were placed inside Petri dishes containing standard fly cultivation medium and raised at $25^{\circ}$ as before. 12 or 24 hours after the first imaging time point the same NMJs were again 
subjected to confocal live imaging. Only NMJs of larvae with a net length increase of more than $10 \%$ (12h interval) or $20 \%$ (24h interval) were accepted for analysis.

\subsubsection{Immunostainings}

Immunostainings of embryonic or larval body-wall preparations were normally visualized with a wide field fluorescence light microscope (Axioskop 2 MOT, Zeiss, Jena, Germany) equipped with an Axiocam camera (plus Axiovision software) and either a $100 x$ oil objective of numerical aperture 1.4 or a $40 x$ oil objective of numerical aperture 1.3. Filters for GFP, Су2, Су3 and Cy5 could be switched manually. The Axioskop 2 MOT system allowed the image recording under fixed illumination conditions. Images were taken with a resolution of $2600 \times 2060$ pixels

(pixel size: $34 \times 34 \mathrm{~nm}$ ). To record z-stacks of whole NMJs confocal microscopy on a Leica NM IRE2 system was performed (see 3.3.2). Here, images were usually taken with a resolution of $2048 \times 1024$ pixels (pixel size: $98 \times 98 \mathrm{~nm}$ ).

The images shown in Fig. 21A,B and Fig. 23E,F were produced by Qin Gang.

\subsubsection{Transmission electron microscopy}

(performed by Carolin Wichmann)

Dissected preparations of $3^{\text {rd }}$ instar larvae (NMJ 6/7, segment A2/A3) were primary fixed in a mixture of $4 \%$ paraformaldehyde and $0.5 \%$ glutaraldehyde in $0.1 \mathrm{M}$ PBS ( $\mathrm{pH}$ 7.2) for 10' and additionally fixed 60' on ice with secondary fixative comprising $2 \%$ glutaraldehyde in $0.1 \mathrm{M}$ sodium cacodylate buffer ( $\mathrm{pH} 7.2$ ). Subsequently, the preparations were washed three times for $5^{\prime}$ in sodium cacodylate buffer and postfixed on ice for $1 \mathrm{~h}$ with $1 \%$ osmium tetroxide (in $0.1 \mathrm{M}$ sodium cacodylate buffer), followed by an $1 \mathrm{~h}$ washing step in sodium cacodylate buffer and three brief washing steps in distilled water. The samples were stained en bloc with $1 \%$ uranyl acetate in distilled water for $1 \mathrm{~h}$ on ice. After a brief rinse with distilled water, the samples were dehydrated at room temperature in increasing ethanol concentrations, infiltrated in Epon resin (100\% EtOH/Epon 1:1, 30' and 90'; 100\% Epon, over night) and embedded for $24 \mathrm{~h}$ at $85^{\circ} \mathrm{C}$. The samples were trimmed, and series of $80-90 \mathrm{~nm}$ ultrathin sections were cut with a $35^{\circ}$ diamond knife (Diatome, Biel, Switzerland) on a Reichert Ultracut Ultramicrotome (Leica, Nussloch, Germany) and mounted on Formvar-coated grids. The sections were stained in uranyl acetate and lead citrate. Pictures were taken with an EM 301 (Philips, Mahwah, USA) transmission electron microscope. 


\subsubsection{Quantitative image analysis}

\subsubsection{In vivo imaging - basic procedure}

Quantitative image analysis was carried out manually using ImageJ $(\mathrm{NIH}$, Bethesda, USA, 6.2B). All attempts to automate the image analysis with other software products proved to be unreliable.

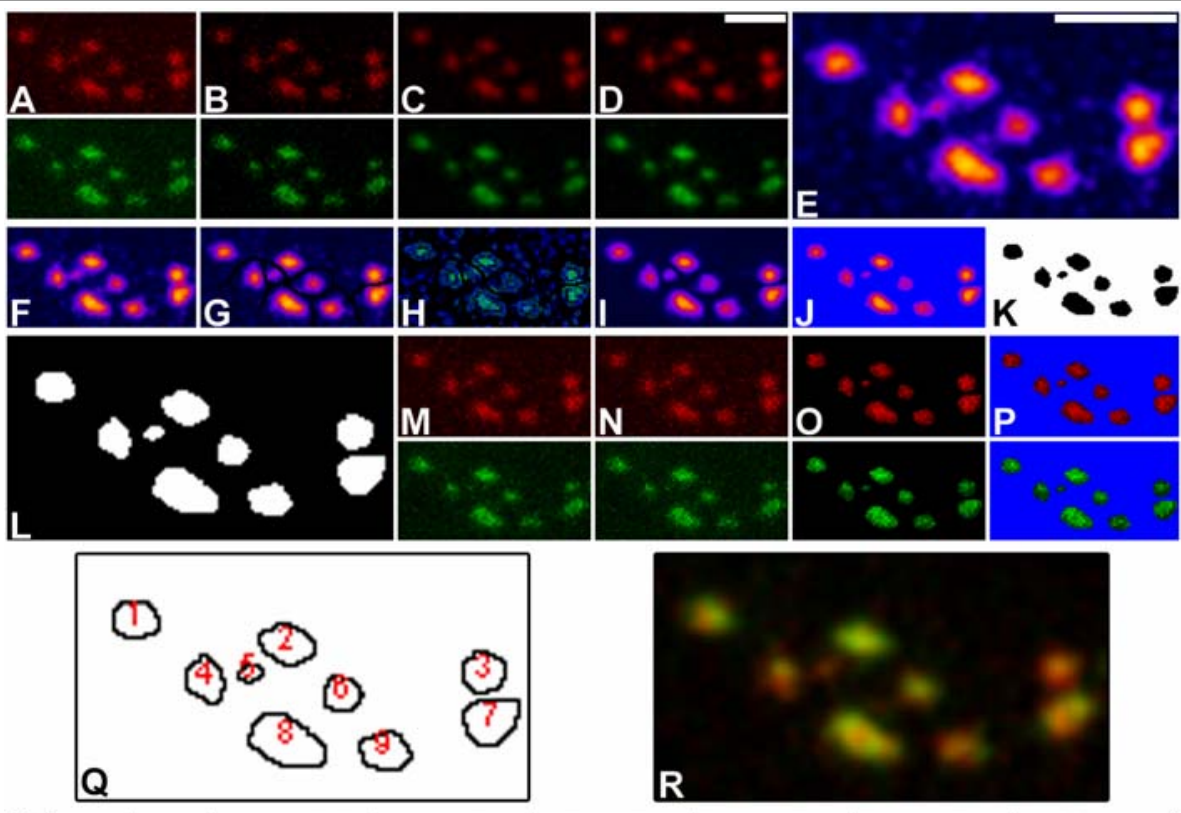

\begin{tabular}{|c|c|c|c|c|c|c|c|}
\hline No. & Pixel & Area $[\mu \mathrm{m} 2]$ & $\begin{array}{l}\text { Intensity } \\
\text { IIB }^{\text {GFP }} \text { abs. }\end{array}$ & $\begin{array}{l}\text { Intensity } \\
\text { IIB }^{\text {GFP }} \text { rel. }\end{array}$ & $\begin{array}{l}\text { Intensity } \\
\|^{\text {mRFP }} \text { abs. }\end{array}$ & $\begin{array}{l}\text { Intensity } \\
\text { IIA }^{\text {mRFP }} \text { rel. }\end{array}$ & $\begin{array}{c}\text { Ratio } \\
\| B^{\text {GFP }} / \text { IIA }^{\text {mRFP }}\end{array}$ \\
\hline 1 & 114 & 0,27 & 88,39 & 1,11 & 91,55 & 0,88 & 1,26 \\
\hline 2 & 143 & 0,34 & 106,99 & 1,34 & 87,64 & 0,84 & 1,59 \\
\hline 3 & 115 & 0,27 & 56,17 & 0,70 & 111,52 & 1,07 & 0,66 \\
\hline 4 & 106 & 0,25 & 60,81 & 0,76 & 93,63 & 0,90 & 0,85 \\
\hline 5 & 20 & 0,05 & 47,20 & 0,59 & 82,35 & 0,79 & 0,75 \\
\hline 6 & 80 & 0,19 & 79,38 & 0,99 & 88,11 & 0,85 & 1,17 \\
\hline 7 & 180 & 0,43 & 80,47 & 1,01 & 106,08 & 1,02 & 0,99 \\
\hline 8 & 242 & 0,58 & 101,32 & 1,27 & 103,87 & 1,00 & 1,27 \\
\hline 9 & 124 & 0,30 & 60,90 & 0,76 & 94,49 & 0,91 & 0,84 \\
\hline
\end{tabular}

\begin{tabular}{|l|l|l|l|}
\hline avrg NMJ & 79,95 & avrg NMJ & 104,09 \\
\hline
\end{tabular}

\section{Fig. 19. Image analysis steps}

$\boldsymbol{A}$, Maximum projections of confocal z-stacks (512x512 pixels). Shown are exemplary in vivo images of GluRIIA $^{\text {mRFP893 }}$ (red) and GluRIIB ${ }^{\text {GFP897 }}$ (green) from $3^{\text {rd }}$ instar larvae expressing both fluorescently tagged subunits in the gluRIIA $A^{\text {null }} I I B^{\text {null }}$ background. $B$, Subtraction of the mean background gray value. $\boldsymbol{C}$, Gaussian blur filtering (radius 1 pixel). $\boldsymbol{D}$, Normalization of the maximum intensity. The maximum gray value was measured and set to 255 via multiplication of the whole image. $E$, Maximum overlay of the red and green channel from $\boldsymbol{D}$. $\boldsymbol{F}$, Scaling of $\boldsymbol{E}$ to $1024 \times 1024$ pixels (with interpolation). $\boldsymbol{G}$, Manual segmentation (line width: 2 pixels) to achieve single PSDs. $\boldsymbol{H}$, Reduced noise after "A trous" wavelet filtering ( $\mathrm{k} 1=50, \mathrm{k} 2=15, \mathrm{k} 3=15, \mathrm{k} 4=\mathrm{k} 5=0$, std dev $=1.5$, noise display activated). I, Signals after "A trous" wavelet filtering. $\boldsymbol{J}$, Threshold set-up to a gray value of $50 . \boldsymbol{K}$, Binary mask after threshold application. $\boldsymbol{L}$, Inversion of $\boldsymbol{K}$. All signals have the gray value 255 , all background pixels have the gray value $0 . \boldsymbol{M}$, Maximum projections from $\boldsymbol{A}$. $\boldsymbol{N}$, Scaling of $\boldsymbol{M}$ to $1024 \times 1024$ pixels (no interpolation). $\boldsymbol{O}$, Minimum overlay of $\boldsymbol{N}$ and the binary mask $\boldsymbol{L}$. $\boldsymbol{P}$, Threshold set-up to a gray value of 1 . In that way, all background pixels are excluded. $\mathbf{Q}$, Outlines of the analyzed particles. All particles consisting of at least eight pixels are included. $\boldsymbol{R}$, RGB overlay of GluRIIA ${ }^{\text {mRF893 }}$ (red) and GluRIIB ${ }^{\text {GFP897 }}$ (green) from $\boldsymbol{D}$. S, Exemplary data of the PSDs from panel $\boldsymbol{R}$. The analysis provides the size (pixels) and the mean gray values of each particle (intensity IIB ${ }^{\text {GFP }}$ and IIA ${ }^{\text {mRFP }}$ abs.). 1 pixel corresponds to $0.00239 \mu m^{2}$. The relative intensities refer to the mean gray values of all analyzed pixels (avrg NMJ). The ratio $\mathrm{IIB}^{\mathrm{GFP}} / \mathrm{IIA} \mathrm{ARFP}^{\mathrm{mRF}}$ arises from the division of the relative intensities. Relating the data to the overlay shown in $\boldsymbol{R}$ confirms the differential particle proportions (e.g. compare the size of particles 4 and 5 ). Furthermore, the varying GluR composition can be demonstrated numerically (e.g. compare the ratios of particles 2 and 3). Scale bars: $2 \mu \mathrm{m}$. 
The multi-step image processing is in detail shown and described in Fig. 19. In short, maximum z-projections of the confocal z-stacks were used to produce a binary mask. Therefore the projections were background-corrected, filtered and normalized. Next, a maximum overlay of both the GFP and mRFP channel was manually segmented to define single synaptic signals. After a further noise reduction a critical threshold was applied, resulting in the binary mask. Finally, the binary mask was superimposed (minimum overlay) with the original maximum projections and the signals were analyzed.

The following measurement parameters were therefore activated: area, mean gray value (from here on referred to as "mgv"), limit to threshold, minimum size: 8 pixel (equivalent to $0.019 \mu \mathrm{m}^{2}$ ), maximum size: not restricted, show outlines, display results, exclude edge particles.

The analysis provided data of all particles about the area and the mean gray values of both the red and green channel. The area, denoted in pixels, was converted to $\mu \mathrm{m}^{2}$ while the absolute mean gray value of each channel and particle was converted to a relative intensity (referring to the mean gray value of all pixels). This allowed numerical data about the GluR composition of single PSDs, expressed by the ratio $r(n)$.

$$
\left.I n t_{G F P}(n)^{r e l}=\frac{m g v_{G F P}(n) * \sum_{1}^{n} A(n)}{\sum_{1}^{n}\left[A(n) * m g v_{G F P}(n)\right]}\right] \operatorname{Int} t_{m R F P}(n)^{r e l}=\frac{m g v_{m R F P}(n) * \sum_{1}^{n} A(n)}{\sum_{1}^{n}\left[A(n) * m g v_{m R F P}(n)\right]}
$$

- $\operatorname{mgv}_{\mathrm{GFP}}(\mathrm{n})$ mean gray value of PSD $n-$ GFP channel

- $\operatorname{mgv}_{\text {mRFP }}(n)$ mean gray value of PSD $n-m R F P$ channel

- $A(n)$ area of PSD n [pixel]

- $\operatorname{Int}_{\mathrm{GFP}}(\mathrm{n})^{\text {rel }} \quad$ relative intensity of PSD $\mathrm{n}$ (to NMJ mgv) - GFP channel

- $\quad \operatorname{Int}_{\text {mRFP }}(n)^{\text {rel }}$ relative intensity of PSD $n$ (to NMJ mgv) - mRFP channel

- $r(n)$ ratio of relative intensities GFP/mRFP channel

$$
r(n)=\frac{\frac{m g v_{G F P}(n) * \sum_{1}^{n} A(n)}{\sum_{1}^{n}\left[A(n) * m g v_{G F P}(n)\right]}}{\frac{m g v_{m R F P}(n) * \sum_{1}^{n} A(n)}{\sum_{1}^{n}\left[A(n) * m g v_{m R F P}(n)\right]}}=\frac{m g v_{G F P}(n) * \sum_{1}^{n}\left[A(n) * m g v_{m R F P}(n)\right]}{\sum_{1}^{n}\left[A(n) * m g v_{G F P}(n)\right] * m g v_{m R F P}(n)}
$$




\subsubsection{In vivo imaging - Tracing and development of single PSDs}

The development of single PSDs was followed within a $12 \mathrm{~h}$ growth interval during the $3^{\text {rd }}$ instar larval stage (see 3.3.2). To start, the consecutive images of $t=0 h$ and $\mathrm{t}=12 \mathrm{~h}$ were analyzed (according to 3.3.5.1) to obtain the pixel area, the mean gray values of both the GFP and mRFP channel and the relative intensity ratio $r$ (GFP/mRFP channel) for all single PSDs.

Next, the PSDs from $t=0 h$ had to be assigned to the PSDs from $t=12 h$. As the "Analyze Particles" option of ImageJ allocates the PSD numbers based on their $y$ position, the PSD numbering of both time points varies. Furthermore, newly formed, additional PSDs can also reform the PSD numbering. Therefore, the assignment had to be performed manually. Only PSDs which could be unambiguously identified at both time points were considered for further analysis. Cases where, e.g. two PSDs converged to one (or the other way round) due to the z-projection were excluded in any event.

The successful assignment made it possible to calculate the absolute and relative changes in PSD area $\left(\triangle A(n),\left[\mu m^{2}\right]\right)$, the relative intensities of the GFP and mRFP channel $\left(\Delta \operatorname{Int}_{\mathrm{GFP}}(\mathrm{n})^{\mathrm{rel}}\right.$ and $\left.\Delta \operatorname{Int}_{\mathrm{mRFP}}(\mathrm{n})^{\mathrm{rel}}\right)$ and the ratio $\mathrm{r}(\Delta \mathrm{r}(\mathrm{n}))$ of single PSDs.

\subsubsection{In vivo imaging - FRAP experiments}

Fluorescence recovery after photobleaching (FRAP) experiments were performed within a time window of $24 \mathrm{~h}$ (see 3.3.2) as the fluorescence recovery of glutamate receptors is very slow (Rasse et al., 2005). The parameters area, relative intensity and ratio $r(n)$ were again determined as outlined above (see 3.3.5.1). Importantly, the PSDs were subdivided into two groups: bleached (bl) and non-bleached (non). The FRAP of the bleached PSDs after $24 \mathrm{~h}$ was calculated as follows identically for both the GFP and mRFP channel:

$$
\begin{aligned}
& \boldsymbol{m g v _ { b l }}(0 h)=\frac{\sum_{1}^{n}[A(n) * m g v(n)]}{\sum_{1}^{n} A(n)} \quad \boldsymbol{m g v _ { n o n }}(0 h)=\frac{\sum_{\sum}^{m}[A(m) * m g v(m)]}{\sum_{1}^{m} A(m)} \\
& m g v_{n o n}(24 h)=\frac{\sum_{1}^{y}[A(y) * m g v(y)]}{\sum_{1}^{n} A(y)} \\
& \operatorname{frap}_{b l}(x, 24 h)=\frac{\frac{m g v_{b l}(x, 24 h)}{m g v_{n o n}(24 h)}}{\frac{m g v_{b l}(0 h)}{m g v_{n o n}(0 h)}}
\end{aligned}
$$


- $\mathrm{n}$

- $\mathrm{m}$

- $\mathrm{mgv}_{\mathrm{bl}}(\mathrm{Oh})$

- $\mathrm{mgv}_{\mathrm{non}}(\mathrm{Oh})$

- $\mathrm{A}(.$.

- $\mathrm{x}$

- $\mathrm{y}$

number of PSDs within the bleached area before bleaching $(\mathrm{t}=0 \mathrm{~h})$

number of PSDs within the non-bleached area before bleaching $(\mathrm{t}=\mathrm{Oh})$

mean gray value of all bleached PSDs before bleaching $(t=0 h)$

mean gray value of all non-bleached PSDs before bleaching $(t=0 h)$ PSD area [pixel]

number of PSDs within the bleached area after recovery $(t=24 \mathrm{~h})$

number of PSDs within the non-bleached area after recovery $(\mathrm{t}=24 \mathrm{~h})$

- $\operatorname{mgv}_{\mathrm{bl}}(\mathrm{x}, 24 \mathrm{~h})$ mean gray value of a single PSD $\mathrm{x}$ within the bleached area after recovery $(\mathrm{t}=24 \mathrm{~h})$

- $\mathrm{mgv}_{\text {non }}(24 \mathrm{~h})$ mean gray value of all non-bleached PSDs after recovery $(\mathrm{t}=24 \mathrm{~h})$

- $\operatorname{frap}_{\mathrm{bl}}(\mathrm{x}, 24 \mathrm{~h})$ FRAP of a single PSD $\mathrm{x}$ within the bleached area after recovery $(\mathrm{t}=24 \mathrm{~h})$

Therefore the achieved recovered intensity of the before bleached PSDs was related to the intensity of the non-bleached PSDs at the time point $\mathrm{t}=24 \mathrm{~h}$. Additionally, intensity discrepancies between the bleached and non-bleached region at $\mathrm{t}=0 \mathrm{~h}$ (before bleaching) were considered to exclude potential local differences in receptor availability.

\subsubsection{NMJ parameters}

Immunostainings of NMJs 4 or $6 / 7$ were used for the quantitative determination of NMJ size, PSD size and PSD/synapse number. Two different protocols were used.

The first measures of both protocols were identical and performed according to the steps shown in Fig. 19. To start, maximum projections of the recorded confocal zstacks (see 3.3.3) were produced, the background was corrected, the images were filtered (Gaussian blur, radius: 2 pixels) and the maximum gray value was normalized to 255 .

Protocol 1 (applied for Fig. 34G,H and Fig. 50A):

The larval NMJ size was quantified from anti-HRP (horse radish peroxidase) immunolabelings of $\mathrm{NMJ} 6 / 7$. Thereto, a threshold of 50 was applied to the processed images and the remaining pixels were quoted and converted to $\mu \mathrm{m}^{2}$. The total PSD number shown in Fig. 34G,H was obtained from antibody (AB) stainings against GFP-tagged glutamate receptors whereas the quantification of the synapse number shown in Fig. 50A resulted from Nc82 immunostainings. In both cases, the 
PSD/synapse number was counted manually with a mechanical counter. Additionally, the segment length of the respective larvae was measured on an Axioskop 2 MOT (see 3.3.3) with a scaled ocular (Zeiss, Jena, Germany) to enable animal size normalization.

Protocol 2 (applied for Fig. 23J,K):

To determine the size of single PSDs (here visualized by PAK immunostainings of NMJ 4) a manual PSD segmentation (see Fig. 19) was necessary. Subsequently, the image was filtered (A trous filter, for settings see Fig. 19) and a threshold of 50 was set. Finally, the remaining particles were counted and analyzed for their pixel number which was converted to $\mu \mathrm{m}^{2}$.

\subsubsection{Comparison of absolute signal intensities}

Wide field fluorescence microscopy (see 3.3.3) at identical stable illumination times was used for image acquisition (image dimension: 2600×2060 pixels). In each case, a cumulative intensity histogram starting from the gray value 255 was produced and the gray value of the $1000^{\text {th }}$ brightest pixel was used to estimate the intensity.

\subsubsection{Determination of intensity profiles}

To quantify the distribution of Fasciclinll relative to PAK (see Fig. 23I), the intensity profile along the maximum diameter of the respective PSD was plotted (profile length, $1 \mu \mathrm{m})$. In each case 20 intensity profiles were put into perspective of the respective maximum absolute intensity and averaged. The Fasciclinll intensity in the PSD centre was determined with respect to the average of the two marginal, perisynaptic intensities.

\subsubsection{Analysis of electron micrographs}

Analysis of synaptic membranes visualized by electron micrographs was performed manually by classifying the pre- and postsynaptic membrane according to their electron-dense character and their linear apposition. SSR thickness was measured as previously described (Gorczyca et al., 1999) from electron micrographs of midbouton sections.

\subsubsection{Statistical analysis}

The nonparametric Mann-Whitney rank sum test was used for statistical analysis of all linear independent data groups. The data are reported as mean \pm s.e.m., $n$ indicates the sample number, and $p$ denotes the significance: ${ }^{*} p<0.05,{ }^{*} p<0.01$, 
${ }^{* * *} p<0.001$. Linear and non-linear (Gaussian fit) regression was used to determine significant data correlation.

\subsubsection{Software}

The image analysis itself was, as mentioned above, led through with Image $\mathrm{J}(\mathrm{NIH}$, Bethesda, USA, see 6.2B). All calculations were performed with Microsoft Excel (Microsoft Corporation, Redmond, USA). Graphics and statistics were produced with Prism 4 (GraphPad Software, San Diego, USA). Image transformation and compilation was done with Adobe Acrobat and Adobe Photoshop (Adobe Systems, San Jose, USA).

\subsection{Electrophysiology}

\subsubsection{Patch clamp recordings}

(performed by Robert J. Kittel)

Mature embryos (stage 17, 20-22h AEL at $25^{\circ} \mathrm{C}$ ) were selected for electrophysiology. All recordings were acquired at $22^{\circ} \mathrm{C}$ from the ventral-longitudinal muscle 6 in anterior abdominal segments $A 2$ and $A 3$, in the whole-cell patch clamp configuration, essentially as previously described (Broadie and Bate, 1993; Featherstone et al., 2000). Miniature EJCs were recorded in extracellular HL-3.1 saline (Feng et al., 2004), consisting of (in $\mathrm{mM}$ ): $\mathrm{NaCl} 70, \mathrm{KCl} 5, \mathrm{MgCl}_{2} 4, \mathrm{NaHCO}_{3}$ 10, trehalose 5, sucrose 115 , HEPES $5, \mathrm{CaCl}_{2}$ 1.5, $\mathrm{pH}$ adjusted to 7.2. Additionally, $2 \mu \mathrm{m}$ TTX was included in the saline to block spontaneous firing of the motoneurons, as the CNS was left attached. The intracellular saline consisted of (in $\mathrm{mM}$ ): $\mathrm{CsCl}$ 158, ATP-NA 2 2, EGTA 5, HEPES 10, pH adjusted to 7.2. The preparation was viewed in transmitted light with an upright microscope (BX51WI, Olympus, Hamburg, Germany) and a 60x water-immersion lens. The patch pipettes were pulled from borosilicate glass $(1.5 \mathrm{~mm}$ outer diameter, with filament) and fire-polished to final resistances of $3-5 \mathrm{M} \Omega$. The input resistance of the muscle ranged from about $600 \mathrm{M} \Omega$ to $2 \mathrm{G} \Omega$, and the series resistance, measured throughout the experiment, was typically between 10 and $20 \mathrm{M} \Omega$. To record mEJCs, the membrane potential was clamped at $-60 \mathrm{mV}$. A single recording lasted for at least 3 minutes, during which the holding current never exceeded $\pm 25 \mathrm{pA}$. The signals were amplified with an Axopatch 200B (Molecular Devices, Sunnyvale, USA) patch-clamp amplifier, recorded at a sampling rate of $10 \mathrm{kHz}$ and low-pass filtered at $2 \mathrm{kHz}$. Only events of an amplitude $\geq 20 p A$ were used for subsequent analysis in Clampfit 9 (Molecular Devices, Sunnyvale, USA). 


\subsubsection{Intracellular recordings}

(performed by Andreas Frölich and Robert J. Kittel)

Two-electrode voltage clamp (TEVC) recordings were obtained from late third instar male larvae (muscle 6, segment A2 or A3), in principle as formerly reported (Kittel et al., 2006). The composition of the extracellular hemolymph-like saline (HL-3) (Stewart et al., 1994) was (in $\mathrm{mM}$ ): $\mathrm{NaCl} 70, \mathrm{KCl} 5, \mathrm{MgCl}_{2} 20, \mathrm{NaHCO}_{3} 10$, trehalose 5, sucrose 115, HEPES 5, $\mathrm{CaCl}_{2} 1, \mathrm{pH}$ adjusted to 7.2. Nerve-evoked EJCs (voltage clamp at $-60 \mathrm{mV}$ ) were recorded with intracellular microelectrodes filled with $3 \mathrm{M} \mathrm{KCl}$ to give final resistances of $12-20 \mathrm{M} \Omega$.

\subsection{Styryl dye labeling}

FM5-95 labeling was done as previously denoted (Kuromi and Kidokoro, 2002; Rasse et al., 2005). In short, styryl dye uptake was induced by nerve stimulation with $30 \mathrm{~Hz}$ for 5 ' in the presence of 20 MM FM5-95 (T-23369, Molecular Probes, Eugene, USA) in normal saline. After stimulation, the preparations were washed three times with $\mathrm{Ca}^{2+}$-free saline ( $2 x$ shortly, then $\left.1 \times 15 \mathrm{~min}\right)$. To destain, high- $\mathrm{K}^{+}$saline was applied for $5 \mathrm{~min}$. 


\section{Results}

\subsection{Non-NMDA type glutamate receptors are essential for maturation but not for initial assembly of synapses at Drosophila NMJs}

\subsubsection{Formation of glutamatergic NMJ synapses deprived of postsynaptic glutamate receptors}

Previous work has identified a total of five glutamate receptor subunits (Fig. 20A, scheme) within Drosophila muscles (GluRIIA, IIB, IIC, IID and IIE), from which two receptor complexes incorporating GluRIIC, GluRIID and GluRIIE together with either GluRIIA or GluRIIB seem to form (Fig. 20B). GluRIIA or GluRIIB containing complexes co-exist within individual synapses of the NMJ. In both gluRIIA or gluRIIB single mutants, structurally normal synapses form, meaning that either complex is per se dispensable for the formation of proper NMJ synapses (Petersen et al., 1997; DiAntonio et al., 1999; Marrus et al., 2004; Chen et al., 2005; Qin et al., 2005). In gluRIIA\&IIB double mutants (gluRIIA ${ }^{\text {null }} I I B^{\text {null}}$ ), however, and similarly in gluRIIC ${ }^{\text {null, }}$ gluRIID ${ }^{\text {null }}$ and gluRIIE ${ }^{\text {null }}$ single mutants, all glutamate receptor subunits are absent from the NMJ resulting in embryonic lethality (Petersen et al., 1997; Featherstone and Broadie, 2002; Marrus et al., 2004; Qin et al., 2005). However, already minimal amounts of the relevant glutamate receptors can rescue the lethality and even give rise to adult flies (Marrus and DiAntonio, 2004; Qin et al., 2005).

To start, effects of glutamate receptor deprivation were studied at the well-described larval NMJ. Here, individual synapses are considerably larger than in the embryo (Rheuben et al., 1999) and, moreover, a recent in vivo imaging study indicated a rate-limiting role for glutamate receptor incorporation in the formation of synapses at larval NMJs (Rasse et al., 2005). Three different situations combining a severe depression of glutamate receptor subunits still compatible with larval vitality have been described previously. When gal4-inducible cDNA constructs of either gluRIIC (Marrus and DiAntonio, 2004) or gluRIID (Qin et al., 2005) were brought into the corresponding single mutant background, "leaky expression" permitted larval survival in the absence of gal4-drivers. A strong reduction in the synaptic expression of all glutamate receptor subunits was observed for the gluRIIC hypo (Fig. 20D) (Marrus and DiAntonio, 2004) and gluRIID hypo (Qin et al., 2005) situation. In the third constellation, the otherwise lethal gluR $\left\|A^{\text {null }}\right\| B^{\text {null }}$ situation could be rescued with a gluRIIA genomic transgene encoding the whole open reading frame but lacking parts of the $3^{\prime}$-UTR (from here on referred to as gluRIIA $A^{\text {hypo }}$ ), resulting in less than $5 \%$ of wild type GluRIIA mRNA levels and certainly no GluRIIB (Qin et al., 2005). Some PSDs obviously still showed normal size and glutamate receptor intensity at gluR/IC hypo NMJs (Fig. 20D, arrow) (Marrus and DiAntonio, 2004). However, at larval NMJs of 
gluRIIA hypo no such PSDs could be observed (Fig. 20E). In fact, only when using atypically long exposure times very faint residual accumulations of GluRIIA and GluRIIC could be visualized (Fig. 20F). Thus, because of their extreme and consistent reduction of glutamate receptors, gluRIIA hypo larvae were used to study the formation of glutamatergic synapses largely deprived of glutamate receptors.

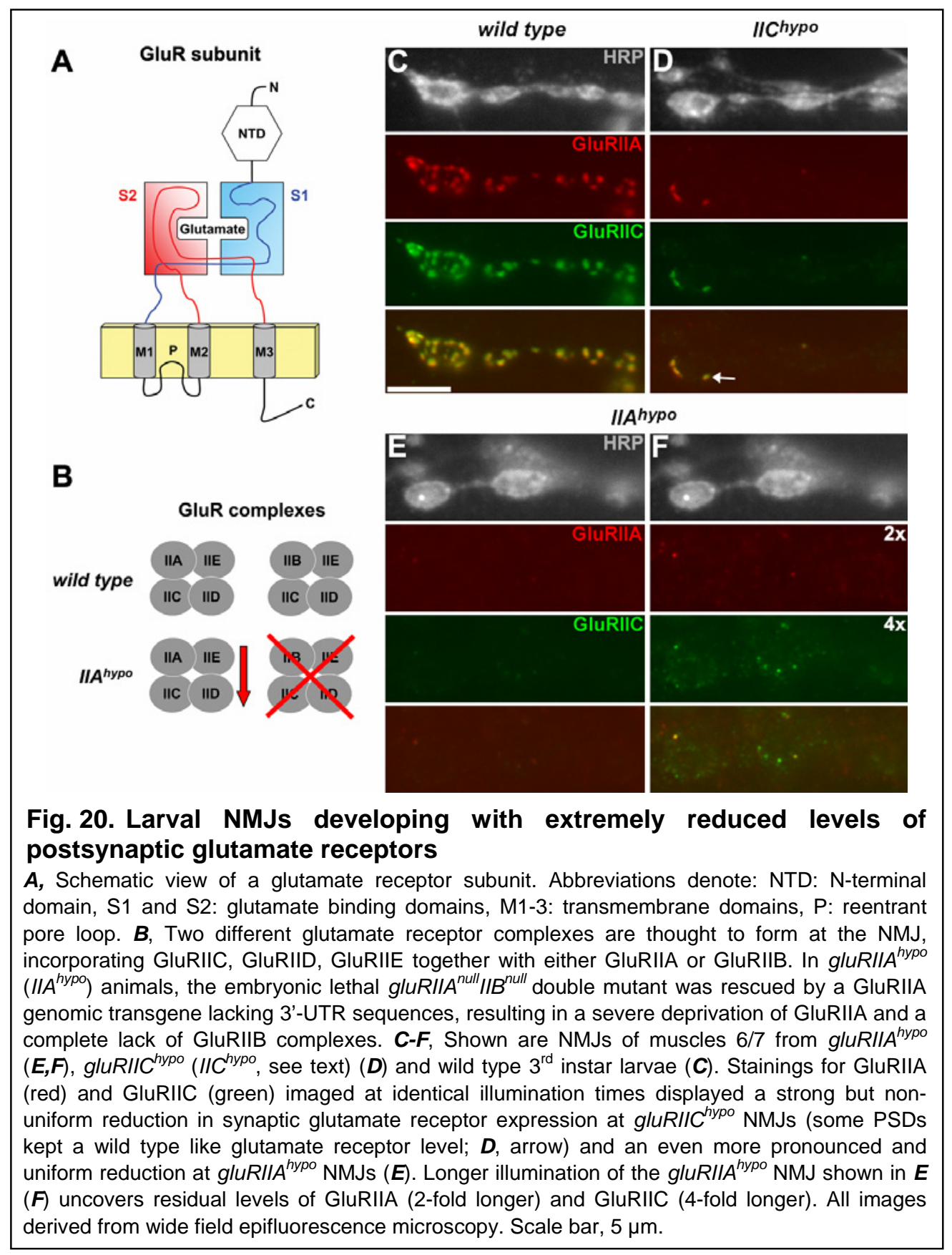




\subsubsection{Presynaptic release sites are functionally and structurally maintained at glutamate receptor deprived NMJ synapses}

$\mathrm{NMJ}$ synapses developing in the near absence of glutamate receptors were further investigated by using molecular markers. The active zone (AZ) is a specialized presynaptic region, where synaptic vesicles dock, fuse, and release their neurotransmitters (Zhai and Bellen, 2004). In Drosophila, AZs are associated with electron-dense specializations (T-bars) (Atwood et al., 1993; Zhai and Bellen, 2004). The monoclonal antibody Nc82 was shown to label the AZs of Drosophila synapses (Wucherpfennig et al., 2003) by recognizing the Bruchpilot protein (BRP), which is essential for T-bar formation (Atwood, 2006; Kittel et al., 2006; Wagh et al., 2006).

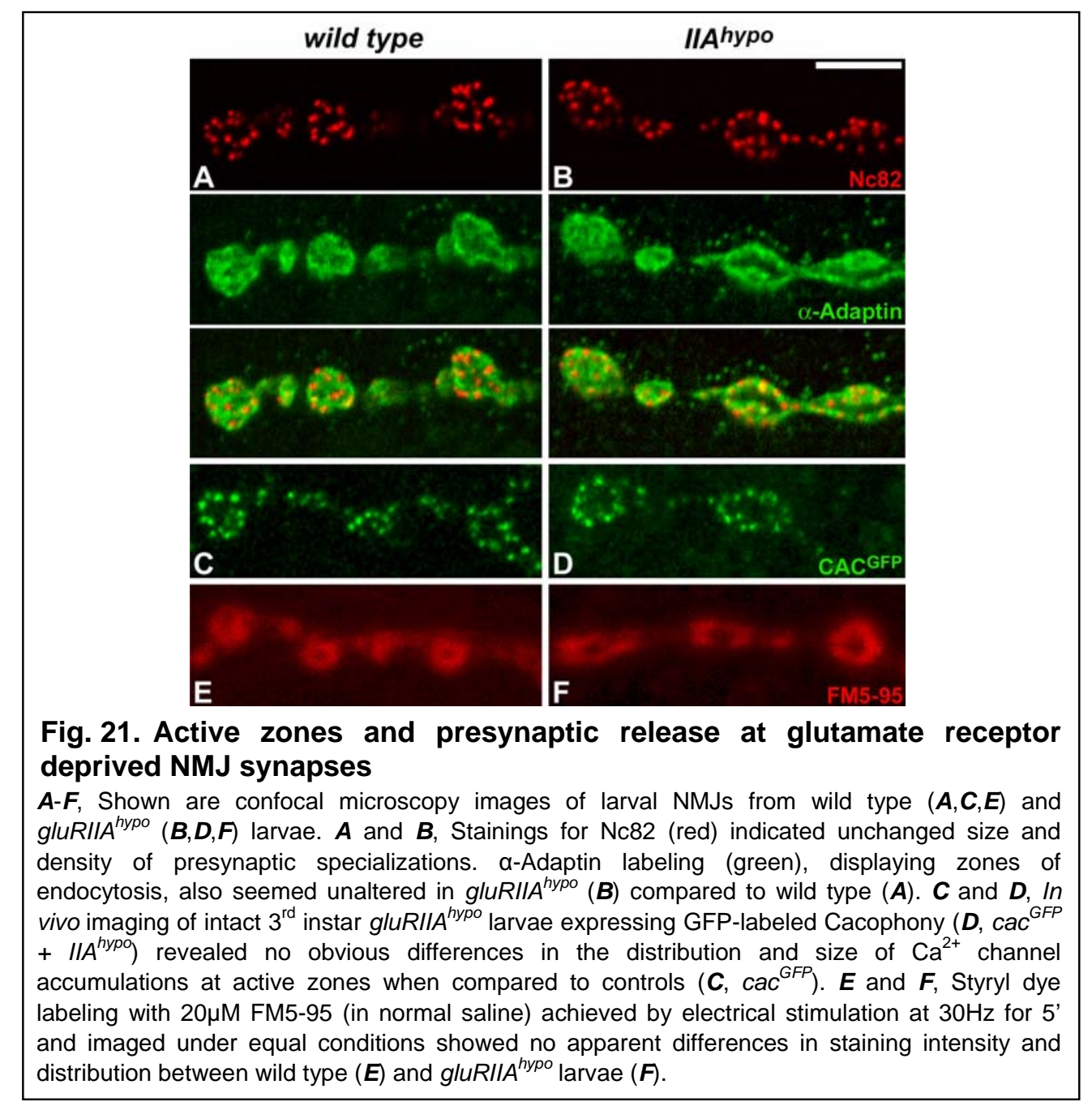

At gluRIIA hypo synapses, the density and size of synaptic clusters of both Nc82 (Fig. 21B) and $\mathrm{Ca}^{2+}$-channels (Fig. 21D) appeared unaffected when compared to wild type controls (Fig. 21A,C). Thus, consistent with the presence of T-bars (see below in Fig. 24), AZ structures seemed to establish normally at gluRIIA hypo synapses. AZs are 
also surrounded by zones of endocytosis, which can be labeled in $\alpha$-Adaptin stainings (Dornan et al., 1997). The distribution of $\alpha$-Adaptin appeared unchanged as well (Fig. 21B). Thus, in contrast to the PSD (see below in Fig. 23 and Fig. 24), the molecular and structural composition of the presynaptic AZs seemed unaffected.

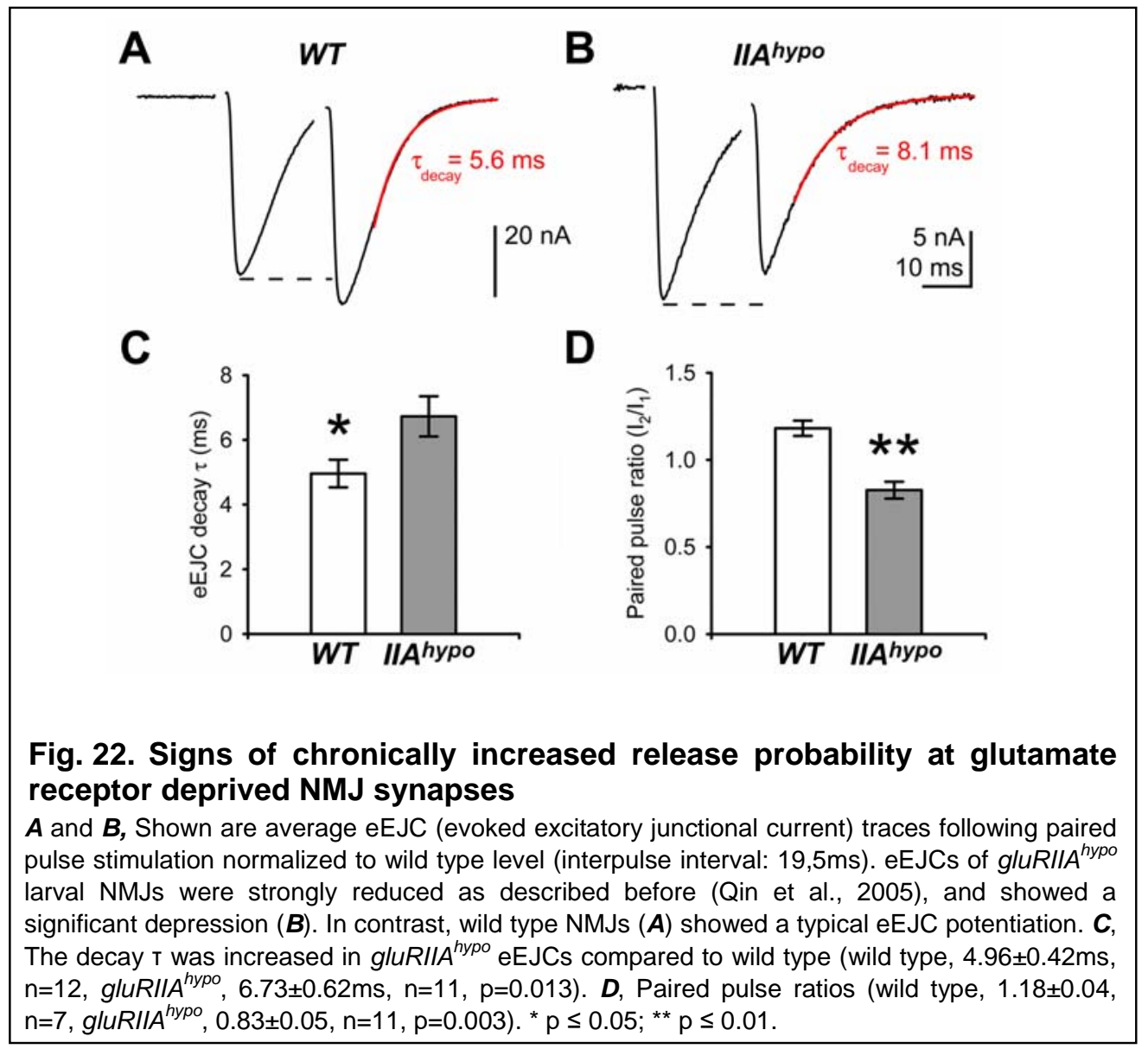

Are these AZs still functional? It could be observed that gluRIIA $A^{\text {hypo }}$ larvae were only moderately limited in mobility. Moreover, styryl dye (FM5-95) incorporation after high frequency stimulation (Kuromi and Kidokoro, 2002; Wucherpfennig et al., 2003) showed that vesicle release persisted at gluRIIA hypo NMJs (Fig. 21F). In a previous electrophysiological analysis it was shown that nerve evoked EJCs at gluRIIA $A^{\text {hypo }}$ NMJs are reduced to about $30 \%$ of the wild type level. Notably, in this genotype miniature EJCs, indicating the postsynaptic glutamate sensitivity at individual synapses, were below the detection threshold, which can be estimated to be at about $20 \%$ of the wild type amplitude (Qin et al., 2005). Thus, following an action potential the number of presynaptically released vesicles is likely increased as part of a presynaptic compensation for reduced postsynaptic sensitivity (Petersen et al., 1997; Reiff et al., 2002). In fact, paired pulse stimulation at these junctions led to an atypical depression as would be expected for a synaptic system with a chronic 
increase in presynaptic release probability (Fig. 22A,B; wild type, $1.18 \pm 0.04, n=7$, gluRIIA $\left.{ }^{\text {hypo }}, 0.83 \pm 0.05, \mathrm{n}=11, \mathrm{p}=0.003\right)$. In addition, the decay time constant ( $\mathrm{T}$ ) of evoked responses was increased (Fig. 22A,B; wild type, 4.96 $\pm 0.42 \mathrm{~ms}, \mathrm{n}=12$, gluRIIA hypo $, 6.73 \pm 0.62 \mathrm{~ms}, \mathrm{n}=11, \mathrm{p}=0.013$ ), potentially pointing towards changes in glutamate clearance or atypical functional properties of the glutamate receptors remaining at these synapses. The rise time of evoked junctional currents was not significantly altered (wild type, $1.08 \pm 0.05 \mathrm{~ms}, \mathrm{n}=12$, gluR/IA $A^{\text {hypo }}, 1.17 \pm 0.08 \mathrm{~ms}, \mathrm{n}=11$, $\mathrm{p}=0.498$ ). In summary, AZs still formed at the presynaptic site of NMJ synapses deprived of glutamate receptors. These AZs appeared fully active in vesicle release, and likely vesicle release is even increased to compensate for the drastically reduced postsynaptic glutamate sensitivity. Such a compensation was described before for gluRIIA mutants, which notably show a less drastic drop in postsynaptic glutamate sensitivity (Petersen et al., 1997).

\subsubsection{Early stop of postsynaptic maturation at glutamate receptor deprived NMJ synapses}

Next, postsynaptic assembly at glutamate receptor deprived synapses was examined. The PAK kinase forms a complex with PIX and Rac, involved in aspects of PSD assembly (Albin and Davis, 2004). PAK widely serves as a PSD marker at NMJ synapses and strictly co-localizes with the glutamate receptor subunit GluRIIA (Rasse et al., 2005). The size of PAK signals at individual PSDs seemed strongly reduced in gluRIIA $A^{\text {hypo }}$ animals (e.g. compare Fig. $23 C$ to D). In fact, quantification of gluRIIA $A^{\text {hypo }}$ NMJs of mature $3^{\text {rd }}$ instar larvae showed a significant reduction in the size of PAK signals (Fig. 23J; wild type, $0.385 \pm 0.005 \mu \mathrm{m}^{2}, \mathrm{n}=1709,6 \mathrm{NMJs}$, gluRIIA ${ }^{\text {hypo }}$, $0.311 \pm 0.006 \mu \mathrm{m}^{2}, \mathrm{n}=1014,7 \mathrm{NMJs}, \mathrm{p}<0.0001$ ), while the density of PSDs (identified as PAK spots) over the NMJ surface appeared unchanged.

In principle, the observed molecular defects in postsynaptic assembly might not reflect a genuine inability to form mature PSDs but instead a deficit in maintenance of matured PSDs (and thus "defective synapses" would have accumulated until late larval development as predominantly analyzed in this study). However, also earlier during development, in $1^{\text {st }}$ (not shown) and $2^{\text {nd }}$ instar gluR IIA $A^{\text {hypo }}$ larvae, synaptic PAK signals were identically reduced (Fig. $23 \mathrm{H}, \mathrm{K}$; quantification for $2^{\text {nd }}$ instar: wild type, $0.358 \pm 0.007 \mu \mathrm{m}^{2}, \mathrm{n}=699,6 \mathrm{NMJs}$, gluRIIA hypo $, 0.280 \pm 0.006 \mu \mathrm{m}^{2}, \mathrm{n}=622,6 \mathrm{NMJs}$, $\mathrm{p}<0.0001)$. 


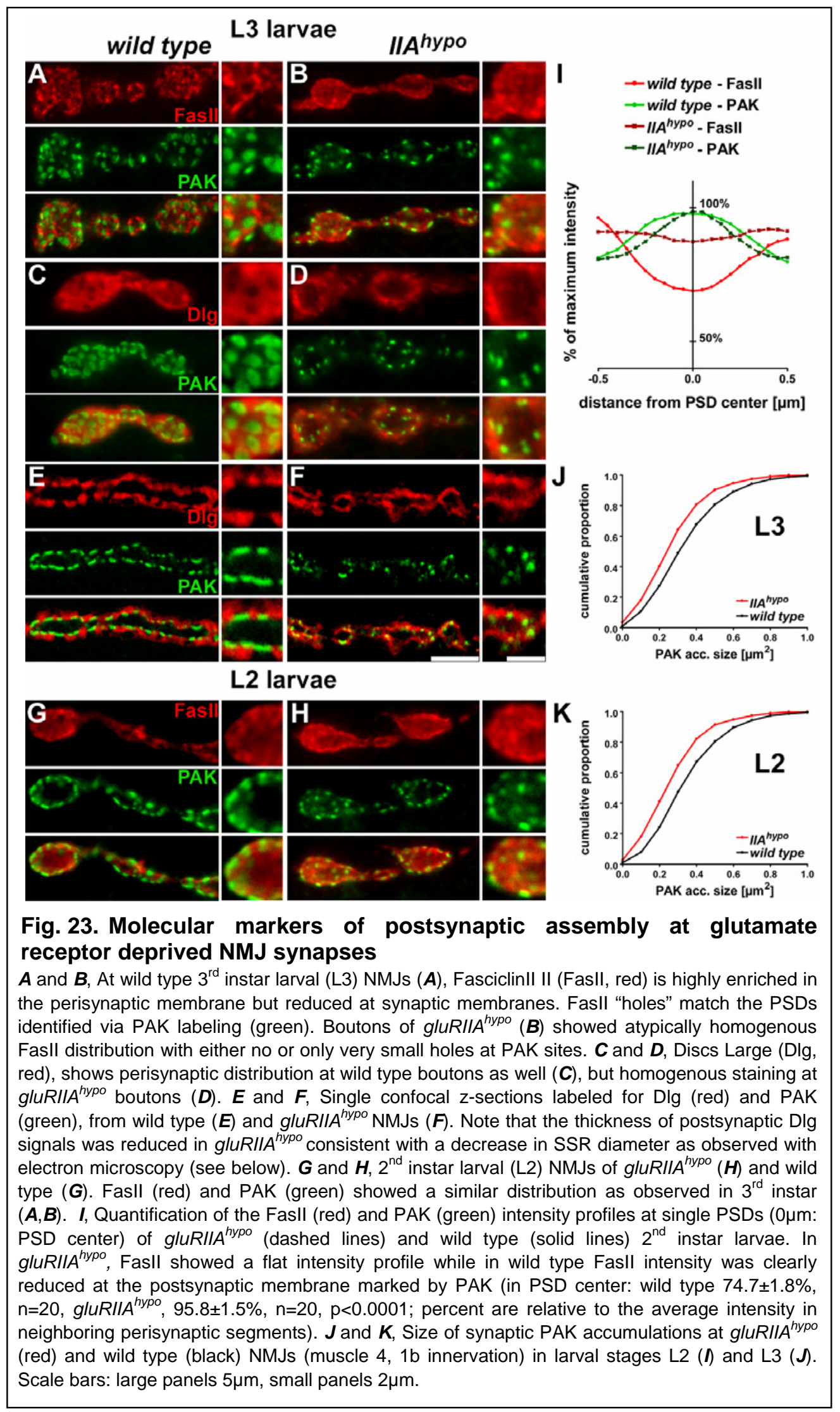


Reduced synaptic PAK signals pointed towards defects in the molecular and/or structural assembly of the PSD region of synapses lacking glutamate receptors. FasciclinII II (FasII), an NCAM-related cell adhesion molecule, and Disc Large (Dlg), founding member of the PSD-95-type MAGUK family take part in growth and maturation of the NMJ structure and interact molecularly (Schuster et al., 1996; Thomas et al., 1997; Zito et al., 1997). At wild type NMJs, Fasll and Dlg are highly enriched at the "perisynaptic" muscle membrane but are clearly reduced at the actual postsynaptic membrane (Fig. 23A,C,G). In gluRIIA hypo larvae, however, Fasll and DIg did not appear reduced at postsynaptic membranes (Fig. 23B,D,H). In fact, quantification of Fasll (Fig. 23I) demonstrated a distinct reduction of Fasll staining intensity at postsynaptic sites (identified by PAK labeling, also note the decreased PAK spot size in gluRIIA $A^{\text {hypo }}$ ) for wild type but a flat distribution at gluRIIA $A^{\text {hypo }}$ synapses (for numbers and details see figure legend).

Hence, perisynaptic proteins such as the membrane protein Fasll and the membrane-associated Dlg are now present in a membrane compartment normally destined to become postsynaptic membrane. It can be concluded that a lack of glutamate receptors interferes with the maturation of postsynaptic sites, and the molecular composition of these postsynaptic assemblies seemed to remain in an immature, nascent state.

\subsubsection{Ultrastructural analysis: lack of apposition between pre- and postsynaptic membranes at NMJ synapses deprived of glutamate receptors}

Defects in NMJ morphology became obvious in immunolabelings of gluRIIA $A^{\text {hypo }} \mathrm{NMJs}$

(Fig. 24C). Both, the number of boutons as well as the number of synapses per NMJ were reduced at gluRIII $A^{\text {hypo }} \mathrm{NMJs}\left(3^{\text {rd }}\right.$ instar, NMJ 4, Ib innervation; bouton number: wild type, $26.7 \pm 2.5, \mathrm{n}=6$, gluR/IA ${ }^{\text {hypo }}, 13.3 \pm 1.9, \mathrm{n}=7, \mathrm{p}=0.0047$; synapse number: wild type, 285 $\pm 16, n=6$, gluR IIA $A^{\text {hypo }}, 153 \pm 17, n=7, p=0.0023$ ). In addition, boutons often appeared abnormally round (Fig. 24C) and no longer polygonal as typically observed in wild type (Fig. 24A).

To further analyze how far postsynaptic differentiation was affected by the lack of glutamate receptors, NMJs of mature gluR/IA $A^{\text {hypo }}$ larvae were subjected to transmission electron microscopy (EM). Within NMJ terminals, synaptic vesicles and typical organelles such as mitochondria seemed unaffected. Moreover, presynaptic T-bars were found at apparently normal frequency (per mid-bouton section: wild type, $0.88 \pm 0.13, \mathrm{n}=8$, gluRIIA $A^{\text {hypo }}, 0.89 \pm 0.26, \mathrm{n}=9, \mathrm{p}=0.96$ ), consistent with the 
preservation of $A Z$ function (see above). T-bars also enabled an unambiguous localization of synaptic sites within electron micrographs.

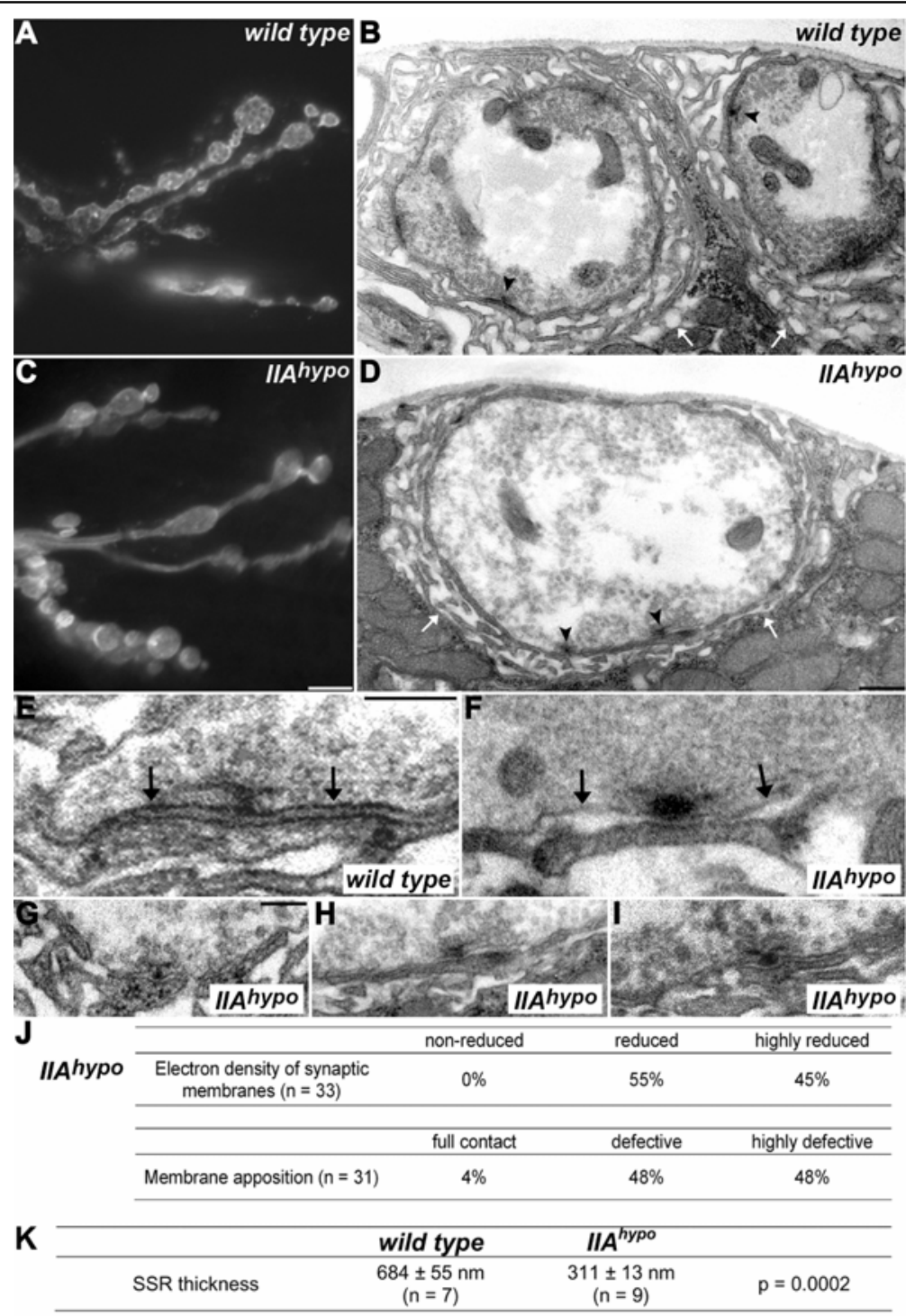

Fig. 24. Electron microscopic analysis of glutamate receptor deprived NMJ synapses

$\boldsymbol{A}$ and $\boldsymbol{C}$, HRP labeling of wild type $(\boldsymbol{A})$ and gluRIIA ${ }^{\text {hypo }}$ NMJs $(\boldsymbol{C})$. Boutons of gluRIIA ${ }^{\text {hypo }}$ tended to be round and were often placed in large intervals along motoneuron branches. $\boldsymbol{B}$ and $\boldsymbol{D}$, Transmission electron micrographs of wild type $(\boldsymbol{B})$ and gluRIIA hypo $(\boldsymbol{D})$ NMJs. Presynaptic T-bars were observed at apparently unchanged density ( $\boldsymbol{B}$ and $\boldsymbol{D}$, arrowheads), while the typical electron-dense character of synaptic membranes $(\boldsymbol{B})$ was reduced at gluRIIA ${ }^{\text {hypo }} \mathrm{NMJs}(\boldsymbol{D})$. The subsynaptic reticulum (SSR) appeared reduced ( $\boldsymbol{B}$ and $\boldsymbol{D}$, white arrows). $\boldsymbol{E}$ and $\boldsymbol{F}$, High magnitude electron micrographs of wild type $(\boldsymbol{E})$ and gluRIIA hypo $(\boldsymbol{F})$ synapses. In wild type, preand postsynaptic membranes are characterized by a close apposition ( $\boldsymbol{E}$, arrows) and electrondense character. At gluRIIA hypo synapses, membranes lacked electron density and linear apposition ( $\boldsymbol{F}$, arrows). G-I, Further examples illustrating ultrastructural defects at gluRIIA hypo synapses. J, Estimation of ultrastructural defects at gluRIIA $A^{\text {hypo }}$ synapses. All analyzed synapses showed moderate or complete loss of electron density, with only one out of 31 gluRIIA ${ }^{\text {hypo }}$ synapses showing linear membrane apposition covering several hundred nanometers as typically observed in wild type ("full contact"). The remaining synapses showed strong or very strong defects in apposition between pre- and postsynaptic membranes. $K$, Quantification of SSR thickness from EM cross sections. All shown images derived from type lb innervations on muscles $6 / 7$ or $12 / 13$ of $3^{\text {rd }}$ instar larvae. Scale bars: C, $5 \mu \mathrm{m}$; D, $500 \mathrm{~nm} ; \mathrm{G}, 200 \mathrm{~nm}$. 
Notably, the glutamate receptor deprived synapses showed severe defects in membrane organization. Normally, pre- and postsynaptic membranes are more electron-dense than neighboring extrasynaptic membranes, and show a flat and linear apposition, easily visualized in EM cross sections (Fig. 24E, arrows). This membrane apposition at mature synapses typically covers a few hundred nanometers, far exceeding the diameter of the attached T-bar (Fig. 24E). At gluRIIA hypo NMJ synapses, however, pre- and postsynaptic membranes showed either no or only reduced electron density (Fig. 24D,F-J). Most notably, the area of close apposition between pre- and postsynaptic membrane was clearly reduced or sometimes totally absent at gluRIIA hypo NMJ synapses (Fig. 24G-J and F, arrows). Instead, the membranes normally destined to show synapse-specific organization were of typical "perisynaptic" organization, which is characterized by the "subsynaptic reticulum" (SSR) that forms by pronounced infolding of the muscle membrane only focally contacting the presynaptic neuronal plasma membrane (Gorczyca et al., 1999). Consistent with the decreased diameter of the Dlg positive area surrounding boutons (Fig. $23 \mathrm{H}$ ), the SSR was reduced in gluRIIA hypo larvae (compare Fig. 24B and D, white arrows; Fig. 24K, SSR thickness, type lb boutons: wild type, 684 $\pm 55 \mathrm{~nm}, \mathrm{n}=7$, gluR/IA ${ }^{\text {hypo }}, 311 \pm 13 \mathrm{~nm}, \mathrm{n}=9, \mathrm{p}=0.0047$ ).

Taken together, it can be concluded that glutamate receptors are directly or indirectly needed to confer proper molecular composition to synaptic membranes. In result, the mature apposition between pre- and postsynaptic membranes normally extending over a few hundred nanometers, and likely acting as a prerequisite for properly timed neurotransmission, could no longer be observed.

\subsubsection{Initial molecular assembly of PSDs independent of glutamate receptors}

Above it was shown, that despite dramatic glutamate receptor deprivation a residual postsynaptic assembly of NMJ synapses demonstrated by PAK localization still took place in gluRIIA hypo larvae. At NMJs of gluRIIA $A^{\text {hypo }}$ embryos, glutamate receptors were not detectable (Fig. 25C), and indistinguishable from gluR $\left\|A^{\text {null }}\right\| B^{\text {null }} N M J s$ in stainings (Fig. 25B). Nevertheless, as shown above, traces of residual glutamate receptor levels could be observed at larval NMJs of gluRIIA $A^{\text {hypo }}$ animals (Fig. 20E,F). In principle, these minimal glutamate receptor levels could be sufficient to establish the observed residual postsynaptic assembly, which in turn could suggest a role of glutamate receptors in initial PSD formation. To address this question, PAK localization was studied at embryonic synapses fully lacking glutamate receptors (stage 17, 20-22h AEL). However, at both gluR/IC null (Fig. 25E) as well as 
gluRIIA ${ }^{\text {null }} / I B^{\text {null }}$ (Fig. 25F) NMJs, PAK still accumulated opposite presynaptic active zones, identified via Nc82 labeling, similar to wild type synapses (Fig. 25D). Similarly, PAK also accumulated at NMJs of gluRIIA hypo embryos (Fig. 25G). Thus, initial molecular assembly at prospective PSD regions still seemed possible in the absence of postsynaptic glutamate receptor complexes.

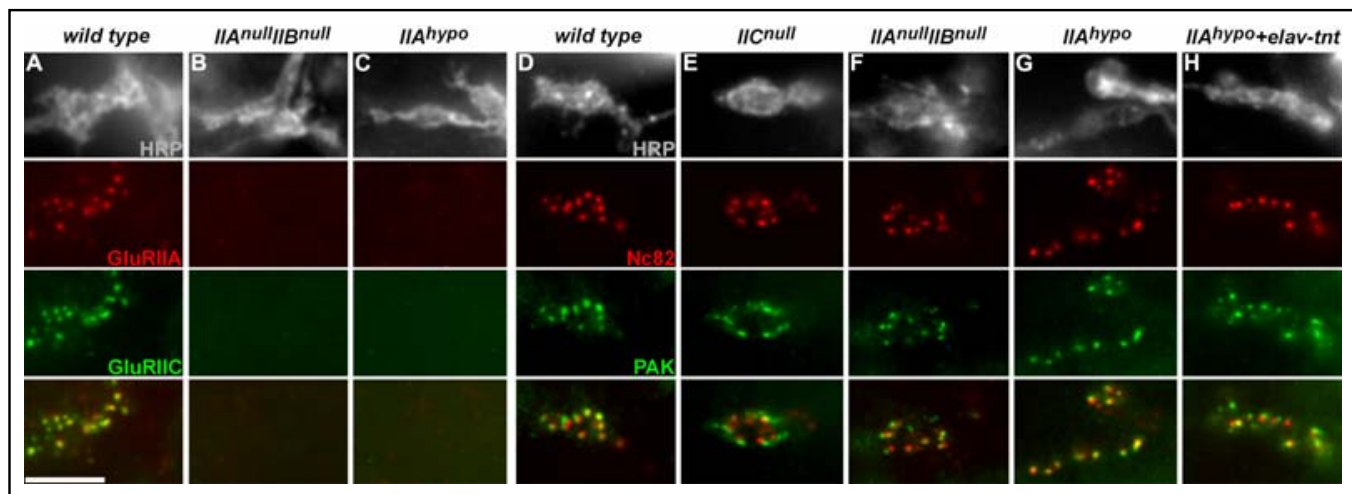

Fig. 25. Synapse assembly at embryonic NMJs lacking all glutamate receptors

$\boldsymbol{A}-\boldsymbol{C}$, Embryonic Drosophila NMJs (stage 17) stained for HRP (grey), GluRIIA (red) and GluRIIC (green). Wild type $(\boldsymbol{A})$ NMJs showed synaptic expression of GluRIIA and GluRIIC while, as expected, glutamate receptors were absent from NMJs of gluRIIA ${ }^{\text {null }} / I B^{\text {null }}$ embryos $(\boldsymbol{B})$. In gluRIIA ${ }^{\text {hypo }}$ embryos $(\boldsymbol{C})$, glutamate receptors were below detection limit as well. $\boldsymbol{D}-\boldsymbol{H}$, Despite the absence of glutamate receptors, embryonic NMJs of gluR $I C^{\text {null }}(E)$ and gluRIIA $A^{\text {null }} \| B^{\text {null }}(\boldsymbol{F})$ animals still showed accumulations of the PSD marker PAK (green) opposite presynaptic release sites labeled with Nc82 (red) similar as in wild type (D). PAK also accumulated at gluRIIA hypo NMJ synapses with $(\boldsymbol{H})$ or without $(\boldsymbol{G})$ a concomitant block of presynaptic activity through panneuronal expression of tetanus-toxin (elav-tnt). Scale bar: $5 \mu \mathrm{m}$.

\subsubsection{Neurotransmission and glutamate-triggered ionic conductance are dispensable for NMJ synapse maturation and growth}

So far, it was shown that glutamate receptors are specifically needed to allow the maturation of the synaptic membrane organization. The question arose, whether the defects at synapses lacking glutamate receptors are mediated by the loss of synaptic transmission, resulting from the absence of glutamate receptors. To check whether a lack of synaptic transmission could in fact be responsible, several independent experimental strategies to block synaptic transmission were chosen. In larvae, tetanus toxin light chain (TNT) was expressed using the mosaic motoneuron driver line ok319-gal4 (Sweeney et al., 1995). Such larvae appeared paralyzed, while in comparison locomotion defects in gluRIIA ${ }^{\text {hypo }}$ larvae were only moderate. However, NMJ synapses of these tetanus toxin expressing larvae had fully developed postsynaptic receptor fields (Fig. 26C). In addition, transgenic expression of temperature-sensitive, dominant-negative Dynamin (UAS-shibire ${ }^{T S 1}$ ) at $29^{\circ} \mathrm{C}$ with the cha-ga/4 driver was used to silence the cholinergic neurons "upstream" of motoneurons (Salvaterra and Kitamoto, 2001). This led to a severe paralysis of 
larvae, while again mature PSDs formed (Fig. 26D). Taken together, a severe blockade of NMJ transmission did not interfere with postsynaptic assembly.

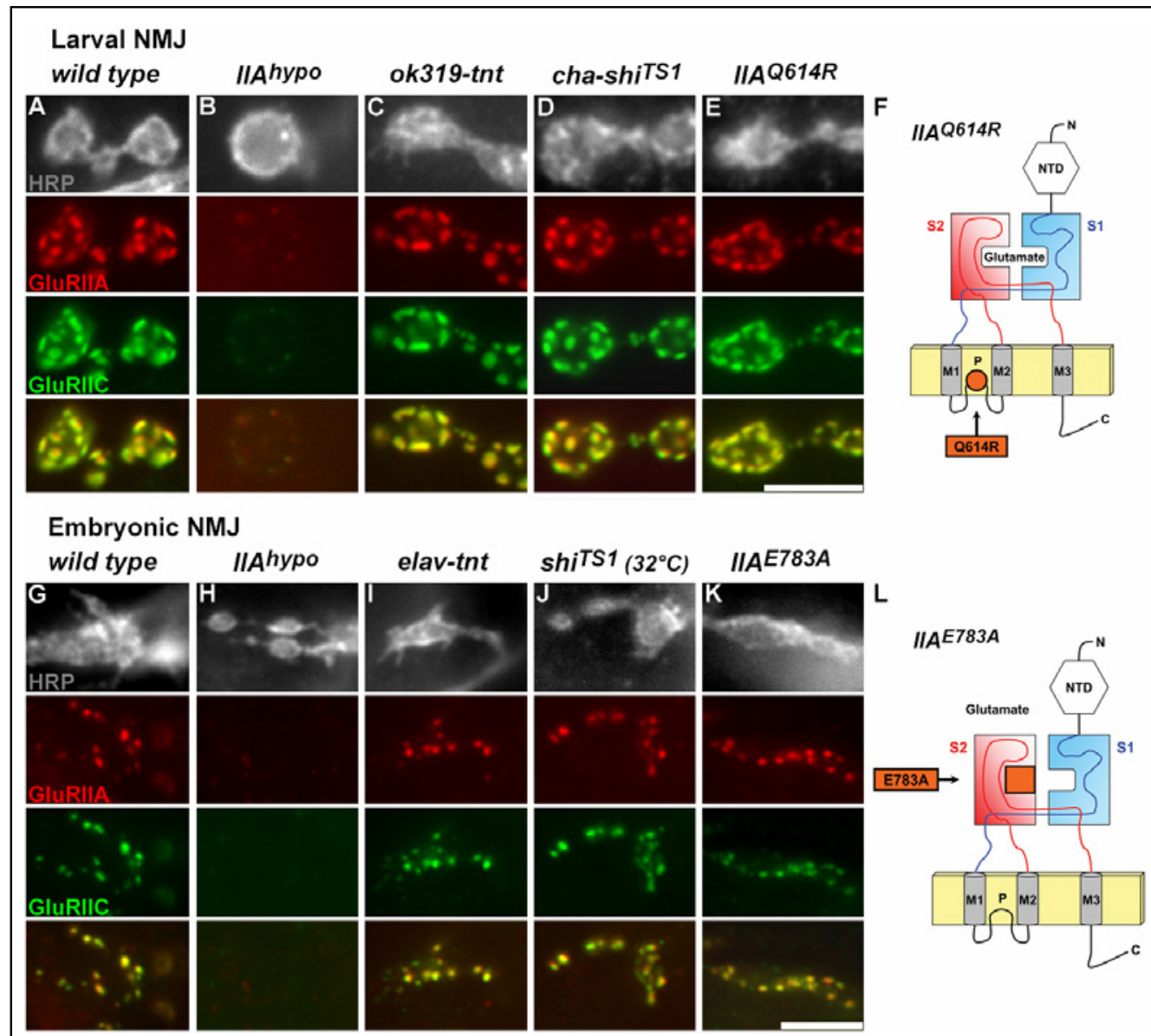

Fig. 26. NMJ synapse assembly after suppression of neurotransmission or glutamate receptor ligand binding

$A-E$, Stainings of $3^{\text {rd }}$ instar NMJs (muscle 6/7) for HRP (grey), GluRIIA (red) and GluRIIC (green). Shown are controls $(\boldsymbol{A})$, gluRIIA ${ }^{\text {hypo }}(\boldsymbol{B})$, larvae expressing either tetanus toxin with the mosaic motoneuron driver ok319-gal4 $(\boldsymbol{C})$ or shibire ${ }^{T S 1}$ in cholinergic neurons $\left(\boldsymbol{D}\right.$, at $\left.29^{\circ} \mathrm{C}\right)$ and a pore modified version of GluRIIA (gluR\|A ${ }^{Q 614 R}, E$ ) expressed in the gluRIIA $A^{\text {null}} \| B^{\text {null }}$ background. $F$, Scheme of GluRIIA ${ }^{\mathrm{Q} 14 \mathrm{R}}$ : a genomic gluRIIA clone with the exchange Q614R.

G-K, Stainings of embryonic NMJs (stage 17) for HRP (grey), GluRIIA (red) and GluRIIC (green). Blockade of synaptic transmission by expression of tetanus toxin light chain with the panneuronal driver elav-gal4 $(I)$ or by raising shibire ${ }^{T S 1}$ animals $(J)$ at restrictive temperature led to embryonic lethality. However, proper clustering of glutamate receptors as in wild type (G) was observed. PSD assembly appeared also unaffected when gluRIIA ${ }^{E 783 A}$ (see $L$ ) was expressed in

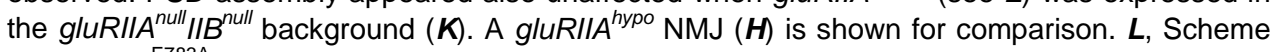
of GluRIIA ${ }^{\mathrm{E} 783 \mathrm{~A}}$, carrying a point mutation in the $\mathrm{S} 2$ glutamate binding domain resulting in embryonic lethality. Scale bar: $5 \mu \mathrm{m}$.

It could be argued that when tetanus toxin expression was driven by ok319-ga/4, suppression of presynaptic release was not complete, as indicated by larval survival. Thus, it cannot be excluded that in particular residual miniature events might be present (Sweeney et al., 1995). In principle, small residual levels of spontaneous activity, as likely present in the tetanus toxin expressing larvae, might already be sufficient to allow postsynaptic assembly. In fact, miniature activity has been 
implicated in the formation of postsynaptic receptor fields in the embryo (Saitoe et al., 2002). However, this finding was discussed controversially (Featherstone and Broadie, 2002; Verstreken and Bellen, 2002). Complete suppression of synaptic release at the NMJ leads to late embryonic lethality in Drosophila. Thus, the consequence of completely suppressing all synaptic transmission including miniature responses at the embryonic NMJ was studied. To this end, a dominant negative allele of Dynamin (shibire ${ }^{T S 1}$ ) which blocks both evoked as well as spontaneous synaptic transmission at restrictive temperature (Koenig et al., 1983) was used. Thereto, after $12-14 \mathrm{~h}(\mathrm{AEL})$ at $25^{\circ} \mathrm{C}$, embryos were transferred to $32^{\circ} \mathrm{C} 8-10 \mathrm{~h}$ before dissection. PSDs (as judged by GluRIIA/GluRIIC co-staining) formed apparently normally in shibire ${ }^{T S 1}$ mutants at restrictive temperature (Fig. 26J). The same result was obtained in embryos expressing tetanus toxin under control of the strong panneuronal driver elav-gal4 (Fig. 26I). Taken together, apparently neither evoked nor spontaneous miniature responses were needed to allow normal postsynaptic assembly. Thus, it appeared unlikely that a general lack of postsynaptic conductance could underlie the postsynaptic defects at glutamate receptor deprived NMJs. In fact, PAK accumulation at PSDs was even possible when a receptor deprived situation (gluRIIA ${ }^{\text {hypo }}$ ) was combined with a concomitant block of activity mediated by tetanus toxin (Fig. 25H).

It can be concluded that ionic conductance through postsynaptic glutamate receptors associated with neurotransmission did not appear to be a prerequisite for synapse assembly. Several studies have measured glutamate in the Drosophila hemolymph (Echalier, 1997). Thus, glutamate receptor conductance in response to such extracellular glutamate, not associated with vesicular release, could per se be implicated in synapse formation. However, genetic constellations meant to increase hemolymph glutamate levels were shown to decrease the size of postsynaptic glutamate receptor fields (Featherstone et al., 2002), arguing against a PSD stabilizing role of such conductances in response to extracellular glutamate. Nonetheless, the role of glutamate mediated receptor conductance was directly

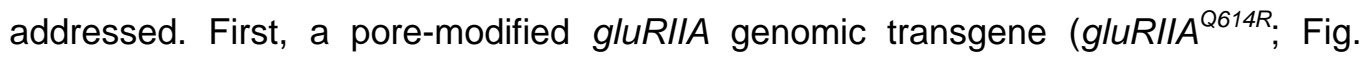
26F), changing the pore from MQQ to MRQ (for AA alignment see Fig. 61) (Jonas and Burnashev, 1995; Kask et al., 1998; DiAntonio et al., 1999; Aronoff et al., 2004) was engineered. This rescued the gluR $I A^{\text {null }} I / B^{\text {null }}$ situation and allowed the formation of apparently normal receptor fields at larval NMJs (Fig. 26E). A "complete block" of $\mathrm{NMJ}$ transmission, however, should result in embryonic lethality, arguing that gluRIIA ${ }^{Q 614 R}$ does still allow some ionic conductance when incorporated into the glutamate receptor complex (DiAntonio et al., 1999). 
In fact, embryonic rescue of the gluRIIA $A^{\text {null }} I I B^{\text {null }}$ situation was no longer possible with a GluRIIA mutated in the glutamate binding pocket (g/uRIIA ${ }^{E 783 A}$, Fig. 26L; for AA alignment see Fig. 60) (Grunwald and Kaplan, 2003). However, at these embryonic gluRIIA ${ }^{E 783 A}$ NMJs wild type like patches of the glutamate receptor subunits GluRIIA and GluRIIC formed at apparently normal density (Fig. 26K). Consistently, PAK kinase and presynaptic BRP also clustered normally at these synapses (not shown), which should be most severely deprived of glutamate-triggered ionic conductance (also given that gluRIIA hypo embryos survive despite the absence of detectable spontaneous responses). In result, a lack of glutamate receptor mediated ionic conductance is most unlikely to be responsible for the PSD defects observed at NMJ synapses lacking glutamate receptors. Instead, glutamate receptors might well be involved in postsynaptic assembly via protein-protein interactions.

\subsubsection{C-terminal truncation of GluRIIA mimics the receptor deprivation defects}

To determine parts of the glutamate receptor proteins involved in synapse assembly and maturation, gluRIIA was deleted from its C-terminus. A truncated genomic transgene (gluRIIA ${ }^{\Delta C 53}$; Fig. 27F), missing the last 53 amino acids of the C-terminus,

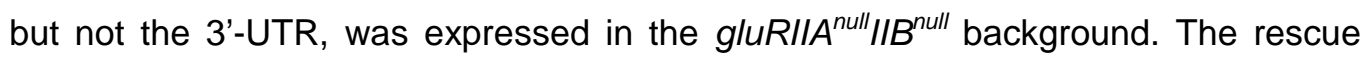
capability of gluRIIA ${ }^{\Delta C 53}$ was lower than observed for gluRIIA $A^{\text {hypo }}(24 \%$ and $43 \%$ of expected Mendelian rate of adult flies, respectively). Similar as found in gluRIIA $A^{\text {hypo }}$ (Fig. 27B), glutamate receptors at gluRIIA $A^{\Delta C 53}$ PSDs (Fig. 27C) were hardly detectable and drastically reduced (different from gluR/lA ${ }^{\text {hypo }}$ with only less than $5 \%$ of GluRIIA mRNA level left, the GluRIIA mRNA level of gluRIIA ${ }^{\Delta C 53}$ was not decreased, not shown). Alongside the reduction in synaptic glutamate receptors, NMJ morphology was clearly defective as well, harboring atypically round boutons (Fig. 27C). PSDs, visualized by labeling PAK, appeared decreased in size. As in gluRIIA ${ }^{\text {hypo }}$, the perisynaptically expressed proteins Fasll and Dlg were essentially evenly distributed over the bouton surface (Fig. 27D,E). Transmission electron micrographs showed a drastic drop in the overall thickness of the SSR (Fig. 27G, arrows; wild type, $684 \pm 55 \mathrm{~nm}, \mathrm{n}=7$, g/uR $/ / A^{\Delta C 53}, 168 \pm 30 \mathrm{~nm}, \mathrm{n}=5, \mathrm{p}=0.0025$ ) and the number of membrane stacks appeared reduced. Importantly, membrane apposition in the synaptic region was only partly established or completely missing, and both pre- and postsynaptic membranes lacked electron-dense character (Fig. $27 \mathrm{H}, \mathrm{I})$. As for gluRIIA hypo, presynaptic elements seemed unaffected (Fig. 27G).

In summary, the gluRIIA ${ }^{\Delta C 53}$ phenotype was very similar to the defects observed for gluRIIA hypo (while even somewhat stronger). These data are consistent with the 
concept that a lack of interactions with other PSD components mediated by the intracellular C-terminus interferes with PSD assembly. However, lack of a C-terminal sequence could per se also affect initial transport and/or assembly of glutamate receptor complexes. In any case, this experiment independently shows that a lack of glutamate receptors interferes with the PSD assembly process.

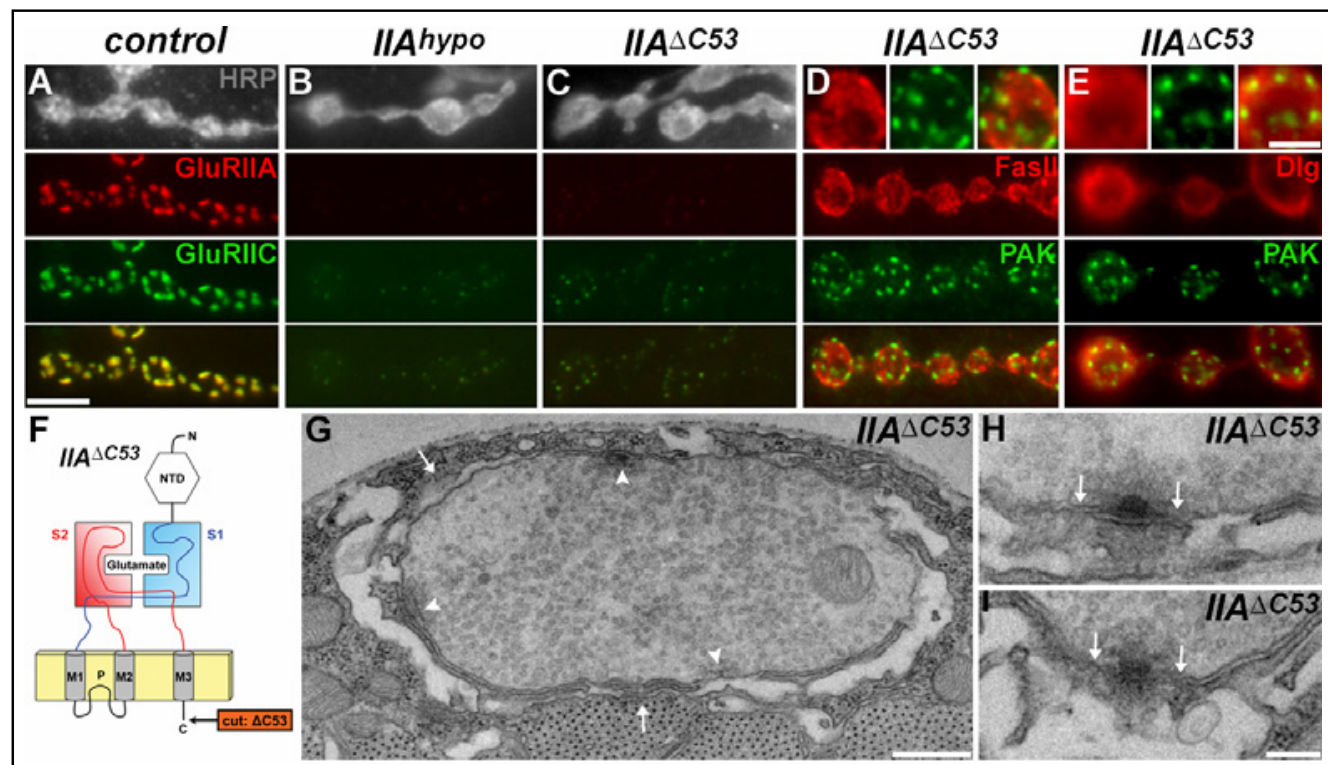

Fig. 27. PSD assembly defects after C-terminal truncation of GluRIIA

$\boldsymbol{A}-\boldsymbol{C}$, Epifluorescence images (recorded with equal illumination time) of $3^{\text {rd }}$ instar NMJs stained for HRP (grey), GluRIIA (red) and GluRIIC (green). Expression of a truncated GluRIIA variant, missing the last 53 amino acids of the C-terminus ( $g / u R / I A^{\Delta C 53}$, see scheme in $\boldsymbol{F}$ ), in the gluRIIA ${ }^{\text {null }} / B^{\text {null }}$ background $(C)$ led to synaptic glutamate receptor levels similar as in gluRIIA $A^{\text {hypo }}$ (B) but severely reduced in comparison to controls (A, wild type genomic gluRIIA construct expressed in gluRIIA $\left.A^{\text {null }} \| B^{\text {null }}\right)$. Boutons at gluRIIA ${ }^{\Delta C 53} \mathrm{NMJs}$ were atypically round as observed in gluRIIA ${ }^{\text {hypo }}(\boldsymbol{B})$. $\boldsymbol{D}$ and $\boldsymbol{E}$, gluRIIA ${ }^{\Delta C 53}$ NMJs stained for Fasll $(\boldsymbol{D}$, red) and Dlg $(\boldsymbol{E}$, red). Similarly to gluRIIA ${ }^{\text {hypo }}$, Fasll and Dlg were no longer restricted from the synaptic membrane but rather evenly distributed over the bouton surface. PAK (green) accumulations were as well clearly decreased in size. $\boldsymbol{F}$, Schematic view of GluRIIA ${ }^{\Delta C 53}$. G, Transmission electron microscopy of gluRIIA ${ }^{\Delta C 53} 3^{\text {rd }}$ instar boutons uncovered a phenotype very similar to gluRIIA hypo. While the formation of presynaptic T-bars persisted (arrowheads), the SSR was strongly reduced in overall thickness (arrows; wild type, 684 $\pm 55 \mathrm{~nm}, \mathrm{n}=7$, gluRIIA $^{\Delta C 53}, 168 \pm 30 \mathrm{~nm}, \mathrm{n}=5, \mathrm{p}=0.0025$ ). $\boldsymbol{H}$ and $\boldsymbol{I}$, Higher magnifications: often complete $(\boldsymbol{I})$ or partial $(\boldsymbol{H})$ lack of the electron-dense character of pre- and postsynaptic membranes could be observed. The synaptic membrane apposition was either fully missing $(\boldsymbol{I})$ or only partly established $(\boldsymbol{H})$ at gluRIIA ${ }^{\Delta C 53}$ synapses (arrows). Scale bars: A, $5 \mu \mathrm{m}$; E, small panels, $2 \mu \mathrm{m}$; G, 500nm; I, 200nm.

\subsubsection{Postsynaptic assembly seems to require intracellular sequence elements common to GIuRIIA, IIB and IIC}

As shown above, the C-terminal deletion gluR $I I A^{\Delta C 53}$ mimicked the gluRIIA $A^{\text {hypo }}$ phenotype. The question remained, which parts of the truncated C-terminal region would be involved in postsynaptic assembly. Therefore, besides gluRIIA ${ }^{\Delta C 53}$, three further genomic transgenes of gluRIIA missing the last 17, 35 and 44 amino acids (AAs) were expressed in the gluRIIA $A^{\text {null } I I B^{\text {null }}}$ background (Fig. 28; gluRIIA ${ }^{\Delta C 17}$, 
gluRIIA ${ }^{\Delta C 35}$ and gluRIIA ${ }^{\Delta C 44}$, respectively). The PSD status was then investigated by glutamate receptor and PAK labeling (Table 3). In gluRIIA ${ }^{\Delta C 17}$, PSDs did not appear smaller than normal, while in gluRIIA ${ }^{\Delta C 35}$ PSD size and glutamate receptor expression level were reduced. gluRIIA $A^{\Delta C 44}$ and similarly gluRIIA ${ }^{\Delta C 53}$ (Fig. 27) showed most severe postsynaptic defects. As mentioned, two glutamate receptor complexes containing either GluRIIA or GluRIIB are co-expressed within individual PSDs of larval NMJ synapses. Absence of both complexes provokes embryonic lethality due to a loss of NMJ transmission (Marrus et al., 2004). Importantly, however, either of both complexes rescues the lethality, resulting in structurally normal PSDs (Petersen et al., 1997; DiAntonio et al., 1999). If protein interactions of the glutamate receptors were instructive, sequences common to both glutamate receptor complexes might well mediate the interactions relevant for PSD assembly.

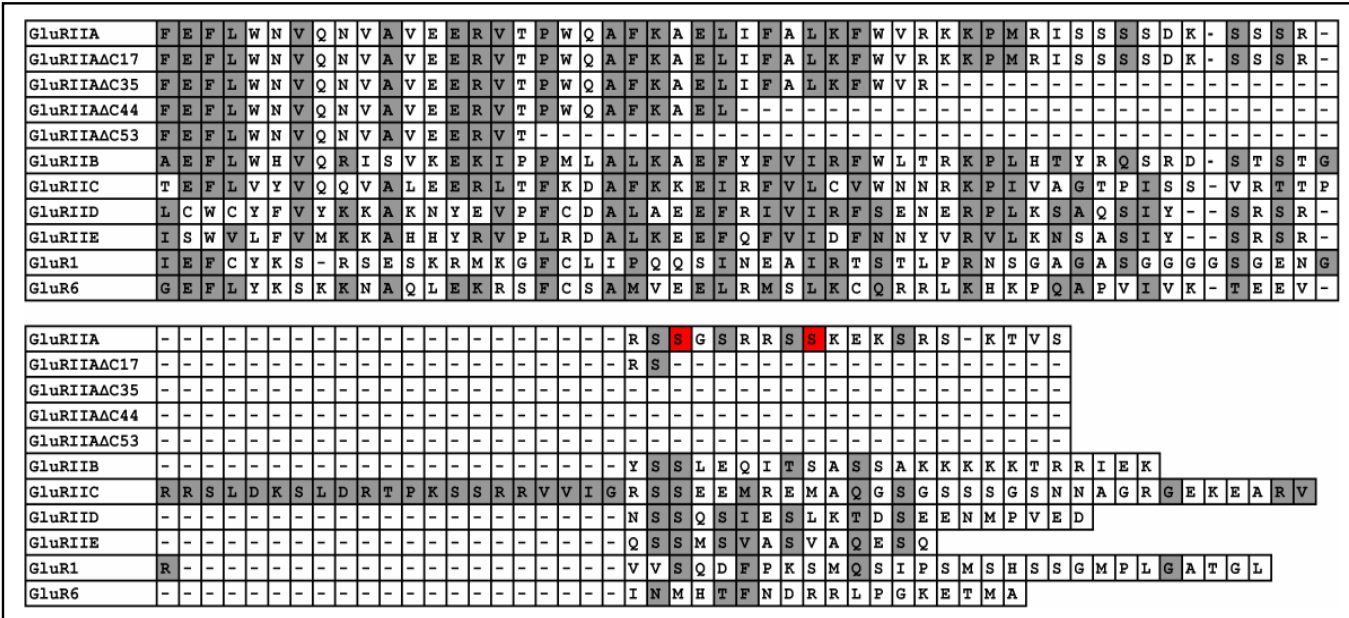

Fig. 28. GluR AA alignment: C-terminal domain (CTD)

Shown are the amino acid (AA) sequences of the Drosophila NMJ glutamate receptor subunits GluRIIA to IIE, the truncated versions of GluRIIA ( $\Delta \mathrm{C} 17, \Delta \mathrm{C} 35, \Delta \mathrm{C} 44$ and $\Delta \mathrm{C} 53)$, the human AMPAR subunit GluR1 and the human kainate receptor subunit GluR6. Conserved residues (based on the chemical AA properties) are highlighted in grey, putative PKA sites (RRXS) on GluRIIA in red.

In fact, it was observed that the region defined as functionally important by the GluRIIA deletion series shows a high sequence similarity to GluRIIB and IIC, but less so to GluRIID and IIE (Fig. 28). Moreover, when the C-terminus of GluRIIB or GluRIIC was placed on GluRIIA, these chimeras rescued the lethality of gluRIIA ${ }^{\text {null }} I I B^{\text {null }}$ double mutants and allowed the formation of proper PSDs. Similarly, GluRIIB with its C-terminus exchanged for the one of GluRIIA (GluRIIB ${ }^{\text {IIA C-term) }}$

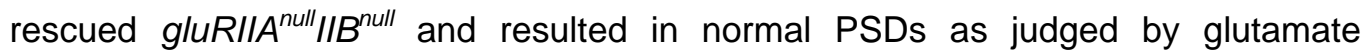
receptor or PAK signal. Moreover, GluRIIC ${ }^{\| A}$ C-term rescued the lethality of the gluRIIC mutant (Marrus et al., 2004), giving rise to apparently normal PSDs. Thus, within the group of GluRIIA, IIB and IIC, which are closest neighbors in terms of similarity (Qin 
et al., 2005), C-termini were exchangeable without affecting the PSD assembly function of these subunits (Table 3). Furthermore, chimera of GluRIIA with GluRIID and GluRIIE, the other group of obligatory subunits of the muscle glutamate receptor complexes, which are only very distantly related to GluRIIA-IIC (Qin et al., 2005), were produced. Both $\mathrm{C}$-terminal exchanges did not allow genetic rescue (Table 3 ). These results are thus consistent with the idea that protein interactions of the Ctermini of the GluRIIA-IIC group to other PSD proteins might be essential for PSD assembly.

\begin{tabular}{|c|c|c|c|c|}
\hline & \multicolumn{2}{|c|}{ Rescue activity } & GluR level & PSD size \\
\hline gluRIIA & $I I A^{\text {null }} B^{\text {null }}$ & + & + & + \\
\hline gluRIIA hypo & $\| A^{\text {null }} B^{\text {null }}$ & - & -- & -- \\
\hline gluRIIA $A^{\Delta C 17}$ & $\| A^{\text {null }} B^{\text {null }}$ & + & + & + \\
\hline gluRIIA $A^{\Delta C 35}$ & $\| A^{\text {null }} B^{\text {null }}$ & - & - & - \\
\hline gluRIIA $A^{\Delta C 44}$ & $\| A^{\text {null }} B^{\text {null }}$ & -- & -- & -- \\
\hline gluRIIA $A^{\Delta C 53}$ & $\| A^{\text {null }} B^{\text {null }}$ & -- & -- & -- \\
\hline gluRIIA $A^{\text {IIB C-term }}(A A B)$ & $\| A^{\text {null }} B^{\text {null }}$ & + & + & + \\
\hline gluRIIA IIC C-term $(A A C)$ & $\| A^{\text {null }} B^{\text {null }}$ & + & + & + \\
\hline gluRIIA IID C-term $(A A D)$ & $\| A^{\text {null }} B^{\text {null }}$ & 0 & n.a. & n.a. \\
\hline gluRIIA IIE C-term $(A A E)$ & $\| A^{\text {null }} B^{\text {null }}$ & 0 & n.a. & n.a. \\
\hline gluRIIB ${ }^{\| A}$ C-term $(B B A)$ & $\| A^{\text {null }} B^{\text {null }}$ & + & + & + \\
\hline gluRIIC IIA C-term (CCA) & $\| C^{\text {null }}$ & + & + & + \\
\hline
\end{tabular}

Table 3. C-terminal modifications

Abbreviations denote: + control level, - reduced, -- highly reduced, o none, n.a. not analyzable. 


\subsection{Fluorophore-tagging of glutamate receptor subunits}

A massive deprivation of glutamate receptors resulted in severe ultrastructural synaptic defects characterized by the loss of proper apposition of the pre- and postsynaptic membranes, which is necessary for efficient neurotransmission. While initial PSD assembly was sustained, the maturation of PSDs was specifically inhibited. The PSDs arrested in an immature state with strongly reduced size missing the typical discrimination of peri- and postsynaptic membrane (see 4.1). Hence, glutamate receptors seem to be required for the expansion of PSDs. Indeed, in vivo imaging on the intact Drosophila larval $\mathrm{NMJ}$ has shown that the entry of the glutamate receptor subunit GluRIIA directly correlates with the growth of single PSDs (Rasse et al., 2005). Therefore, GluRIIA harboring a fluorophore insertion in the middle of its intracellular C-terminus (after AA S893, GluRIIA ${ }^{\text {GFP893) }}$ ) was used. Above it was shown that the C-terminal domain of GluRIIA might play a role in establishing proper membrane apposition (Fig. 27). Besides, it was shown for vertebrate AMPARs that the C-terminal domain is needed for receptor trafficking and transport to the synaptic membrane (Malinow and Malenka, 2002). Surprisingly, the C-terminal fluorophore-tagging of GluRIIA did neither interfere with proper receptor complex targeting nor affect physiological channel properties (Rasse et al., 2005). Nevertheless, extracellular tagging would be preferred for various reasons. The most striking argument for a lumenal fusion is the fact that intracellular, C-terminal fluorophore-tagging of C-terminally truncated subunits is logically impossible. It would be interesting to study the dynamic involvement of e.g. the above presented GluRIIA $^{\Delta C 53}$ (see 4.1.7) in PSD maturation. Furthermore, a lumenal fusion would enable the usage of $\mathrm{pH}$-dependent fluorophores as pHluorin to study vesicular glutamate receptor transport (Ashby et al., 2004).

As already mentioned, a previous of our laboratory focused on how the trafficking of GluRIIA organizes synapse formation at the larval NMJ (Rasse et al., 2005). Recently it was suggested that two glutamate receptor complexes incorporating GluRIIC, GluRIID and GluRIIE with either GluRIIA or GluRIIB are expressed at the Drosophila NMJ (Qin et al., 2005). To address how both receptor complexes are involved in in vivo PSD formation and maturation, GluRIIB had to be functionally tagged with EGFP.

Two different approaches, both based on a recent in vitro transposition screen for rat GluR1 (Sheridan et al., 2002) were chosen to tag GluRIIA and GluRIIB. On the one hand, a randomized generation of EGFP fusion proteins by in vitro transposition was performed. On the other hand, functional EGFP insertion sites for rat GluR1 
(Sheridan et al., 2002) were transferred to the corresponding GluRIIA and GluRIIB AA positions.

\subsubsection{Random insertion - In vitro transposition}

The functional tagging of membrane proteins as glutamate receptors is often problematic. The complex 3D structure of glutamate receptor channels might favor fluorophore insertion at the very $\mathrm{N}$-terminus directly after the signal peptide of the respective GluR subunit. In fact, vertebrate AMPARs were successfully labeled at this position (Shi et al., 1999; Perestenko and Henley, 2003). However, all approaches to functionally tag the Drosophila NMJ glutamate receptor subunits GluRIIA and GluRIIB close to their amino terminus failed.

A recent report demonstrated a rapid erratic way to generate ECFP or EGFP insertion libraries which were subsequently screened for positive clones expressing functional fusion proteins via physiological recordings on HEK293 cells (Sheridan et al., 2002).

Here, the in vitro transposition screen protocols were transformed to both GluRIIA and GluRIIB (see 3.1.3). Therefore, the Tn5 transposon TgPT-O carrying both the EGFP and a kanamycin resistance gene flanked by the Tn5 ME mosaic ends was used (Fig. 18). In the presence of $\mathrm{Mg}^{2+}$ the recombinant Tn5 EZ:TNTM transposase catalyzes the random transposon insertion into the target DNA. As target, genomic DNA of gluRIIA and gluRIIB (pSL fa1180fa gluRIIA and pSL1180Nael gluRIIB) was used. The successful transposon integration into the target vector could be detected via ampicillin/kanamycin co-selection as both cloning vectors pSL fa1180fa and pSL1180Nael harbor an ampicillin resistance gene.

For gluRIIA 192 and for gluRIIB 96 colonies were selected and screened for a transposon insertion within the coding region of the respective gene via colony PCR (for primers see 3.1.3). To increase the rate of in frame insertions both 5'-3' and 3'-5' orientation of the transposon was accepted as a subsequent transposon inversion was possible. All clones showing PCR products were sequenced to verify insertions in the correct reading frame.

Assuming that the transposon is integrated randomly into the target DNA, the likelihood for an insertion within the genomic coding region is calculated by dividing the cDNA length by the total plasmid length (both values in bp):

$\begin{array}{ll}\text { gluRIIA } & 2724 \mathrm{bp} / 8760 \mathrm{bp}=31.1 \% \\ \text { gluRIIB } & 2739 \mathrm{bp} / 8301 \mathrm{bp}=33.0 \%\end{array}$


Taking into consideration, that insertions within critical vector regions (as regions required for vector replication as well as the ampicillin resistance gene) might likely inhibit clone formation, the percentages from above increase to $40.4 \%$ and $43.7 \%$, respectively. However, only one third harbors an insertion in the correct reading frame reducing the percentages to $13.5 \%$ for gluRIIA and $14.6 \%$ for gluRIIB.

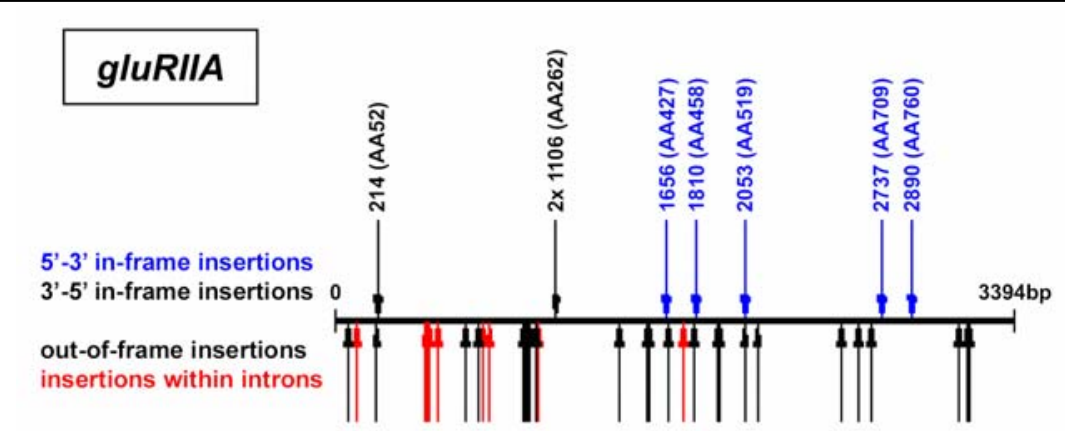

Fig. 29. In vitro transposition results: gluRIIA

50 insertions of TgPT-O within the translated region of gluRIIA (3394bp) could be produced. The correct reading frame was kept by eight insertions (labeled arrows) from which three were 5'-3' (black) and five 3'-5' (blue). The orientation of all 3'-5' insertions could be inverted. 42 insertions (unlabeled arrows) were either out of frame (black) or within introns (red).

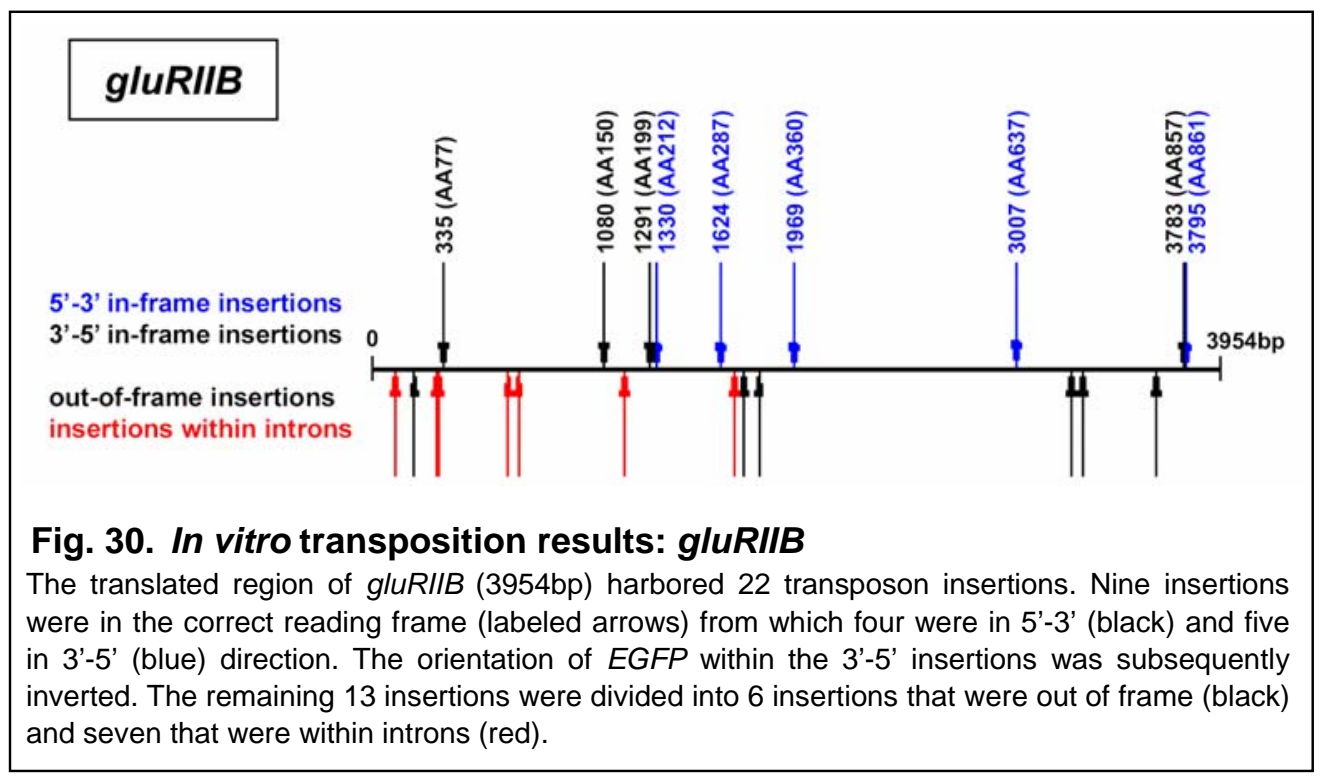

From the 196 clones that were selected from the gluRIIA in vitro transposition reaction (Fig. 29), 72 showed PCR bands. After sequencing only eight clones with an in frame insertion were obtained (4.1\%). 5 of the 8 clones harbored the transposon in 3'-5' orientation. To invert the orientation, a Sall/Ncol fragment was subjected to Ascl restriction and subsequent re-ligation and brought back into the gluRIIA backbone. 32 clones had an insertion out of frame, 10 within introns. The remaining 22 clones were false positive. 
For gluRIIB (Fig. 30) 35 of the 96 selected clones passed the colony PCR screening. 9 clones were positive, 6 had an insertion out of frame and 7 within introns. 13 clones were rated false positive.

The obtained positive insertions resided in the following regions (the number indicates the AA position):

$\begin{array}{lll}\text { gluRIIA }^{\text {GFP }} & \text { NTD } & 52,262(2 \mathrm{x}) \\ & \text { S1/S2 } & 427,458,519,709,760 \\ \text { gluR IIB }^{\text {GFP }} & \text { NTD } & 77,150,199,212,287,360 \\ & \text { M2 } & 637 \\ & \text { CTD } & 857,861\end{array}$

Finally, all positive samples were subjected to Srfl restriction to eliminate the kanamycin resistance gene. The EGFP-tagged transgenes gluRIIA GFP and gluRIIB ${ }^{\text {GFP }}$ were cloned into the expression vector PUAST (Fig. 57) or PUAST XL+ (see 7.1), respectively. The following establishment of transgenic flies was done according to 3.2.2.

All transgenics were tested for their capability to rescue the otherwise embryonically lethal gluRIIA $A^{\text {null } I I B^{\text {null }}}$ double mutant situation (see 3.2.3). Additionally, immunostainings for GFP were performed.

None of the transgenes derived from the in vitro transposition screen rescued the gluRIIA ${ }^{\text {null }} / I B^{\text {null }}$ lethality. Furthermore, neither synaptic nor extrasynaptic GFP signals could be observed (see Fig. 64).

\subsubsection{Site-directed fusion}

\subsubsection{Strategy}

Recently, six functional fluorophore insertion sites could be identified for rat GluR1 (Fig. 31) throughout an in vitro transposition screen. These findings were transformed to Drosophila GluRIIA and GluRIIB. The following respective AA positions could be derived and were chosen as GFP insertion sites:

$\begin{array}{rlllll}\text { rat GluR1: } 211 & \rightarrow & \text { GluRIIA: } & 255 & & \text { GluRIIB: } \\ 261 & \rightarrow & 301 \rightarrow & 284 \\ 284 & \rightarrow & 319 \rightarrow & 309 \\ 324 & \rightarrow & 355 & & \\ 868 & \rightarrow & 893 \rightarrow & 897\end{array}$




\section{Fig. 31. Fluorophore-tagging of rat GluR1}

In vitro transposition reactions carried out with rat GluR1 resulted in six functional ECFP (transposon $T g P T-1$ ) or EGFP (transposon $T g P T-0)$ fusion proteins. While a random insertion of $T g P T-0$ led to the duplication of three amino acids (green), a TgPT-1 insertion is characterized by a duplication of two amino acids (blue) flanking the transposon. The highest number of the respective duplications indicates the exact AA position. Adapted from (Sheridan et al., 2002).

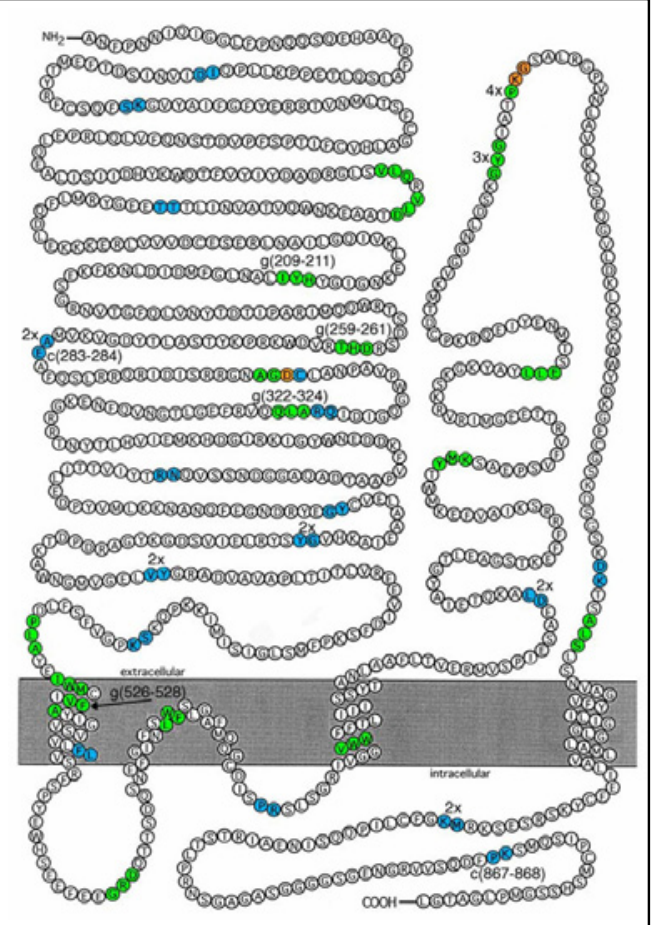

Previously, gluRIIA GFP301 and gluRIIA ${ }^{\text {GFP319 }}$ have been established in our laboratory as cDNA and genomic constructs. Both proved to be non-functional. In this study, gluRIIA ${ }^{\text {GFP255, gluRIIA }}{ }^{\text {GFP355, gluRIIB }}{ }^{\text {GFP284 }}$ and gluRIIB ${ }^{\text {GFP309 }}$ were cloned. The established transgenes did not have any rescue capability for the gluR $I A^{\text {null }} / I B^{\text {null }}$ lethality and showed no detectable GFP signals (Fig. 64).

Recently, gluRIIA was functionally tagged in the middle of the intracellular Cterminus (AA position 893, see 3.1.2.1) and successfully applied to study the organization of synapse formation by in vivo imaging of glutamate receptors (Rasse et al., 2005).

Here, a detailed description is given for the C-terminal tagging of gluRIIB at the analogue AA position 897 (Fig. 17), which demonstrates the principle of all sitedirected GFP fusions.

A genomic Nsil/Ncol fragment of gluRIIB (open reading frame plus $\sim 700 \mathrm{bp}$ upstream and $\sim 500 \mathrm{bp}$ downstream) was used as backbone. The EGFP insert was based on the Tn5 transposon TgPT-O (Fig. 18). In detail (Fig. 32), the last two amino acids before the insertion site were duplicated and the linker regions as well as the Ascl sites were adopted from the Tn5 transposon. To meet the correct reading frame, the $5^{\prime}$ linker was shortened by one base pair as the EGFP started with the $6^{\text {th }}$ base pair. The molecular cloning was performed by a three step overlap extension PCR (Fig. 16) with the restriction sites Nsil/Kpnl flanking the final PCR product (see 3.1.2.1). 
Finally, the GFP-tagged glurlIB ${ }^{\text {GFP897 }}$ was cloned into pUAST XL+ (see 7.1) and transgenics were established in $d f c / h^{4}$ background (see 3.2.2).

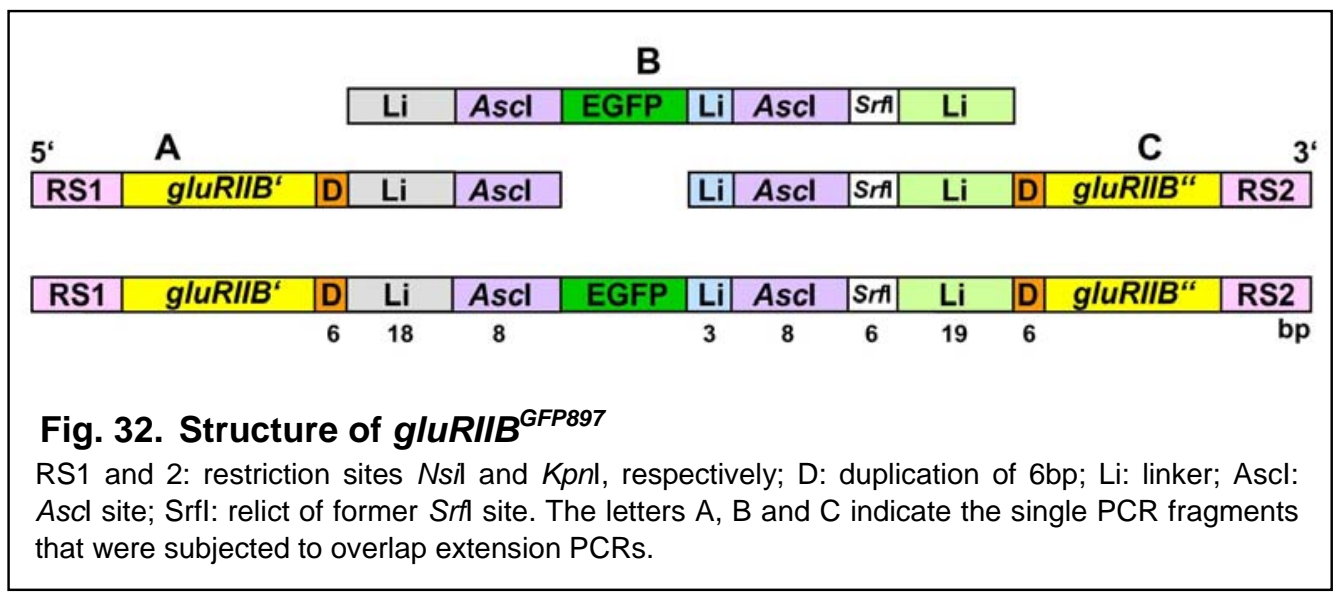

As the EGFP was flanked by Ascl sites it could be easily replaced with mRFP (see 3.1.2.1).

\subsubsection{Outcome}

Anti-GFP immunostainings of $I I B^{G F P}$ larvae (gluRIIB ${ }^{G F P}$ expressed in the otherwise lethal gluRIIA ${ }^{\text {null }} / I B^{\text {null }}$ background) uncovered strong synaptic expression of GluRIIB $^{\text {GFP897 }}$ (Fig. 33). The same could be observed for GluRIIB ${ }^{\text {mRFP897 }}$ (see Fig. 35 and Fig. 64).

Fig. 33. GluRIIB

Larval NMJ of a $I I B^{G F P}$ rescue animal (see Table 2) stained for Fasll and GFP. GluRIIB $^{\text {GFP897 }}$ shows strong and specific postsynaptic expression, surrounded by the perisynaptically expressed Fasll.

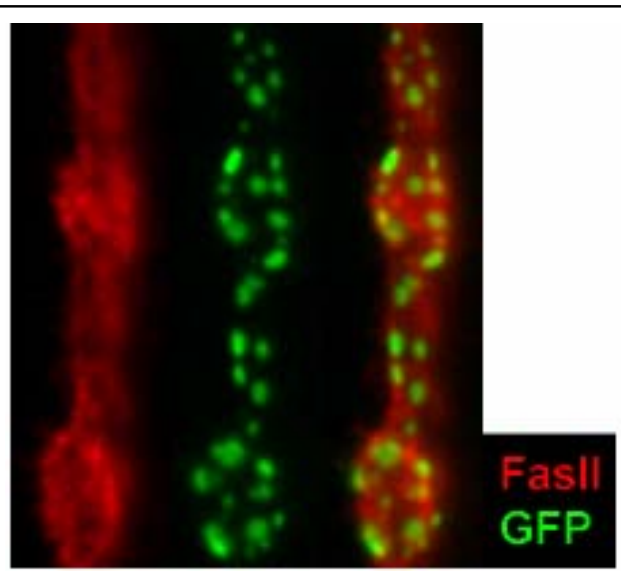

Moreover, gluRIIB ${ }^{G F P}$ was indistinguishable from the untagged gluRIIB concerning rescue capability and physiological properties (for details see 4.3.1). 


\subsection{Subunit-specific targeting of glutamate receptors organizes PSD formation and maturation}

\subsubsection{Functional GFP fusions of GluRIIA and IIB to in vivo study synapse formation at developing NMJs of Drosophila}

Previous work has described two different glutamate receptor complexes expressed at the Drosophila neuromuscular junction, with each complex being sufficient for survival (DiAntonio et al., 1999). Both receptor subtypes contain either the subunit GluRIIA (but not GluRIIB) or GluRIIB (but no GluRIIA), likely together with the subunits GluRIIC, IID and IIE (Fig. 34B) (Schuster et al., 1991; Petersen et al., 1997; Marrus et al., 2004; Featherstone et al., 2005; Qin et al., 2005). As described previously (Marrus et al., 2004), GluRIIA and GluRIIB complexes (from now GluRIIA and GluRIIB) are co-expressed on the level of individual postsynaptic densities, however, were not extensively co-localized within individual PSDs (Fig. 34A). Particularly, small PSDs showed a heterogeneous GluRIIA/GluRIIB composition (often being dominated by GluRIIA, Fig. 34A, arrowheads) while mature size PSDs tended to show a more balanced receptor composition (Fig. 34A, arrows).

Previous studies indicated that GluRIIA and GluRIIB are differentially involved in long-term NMJ plasticity. When GluRIIA was favored over GluRIIB expression, both the number of synapses forming per $\mathrm{NMJ}$ as well as the transmission strength (measured as evoked excitatory junctional current, eEJC) increased. On the contrary, GluRIIB expression antagonized this GluRIIA mediated long-term plasticity (Sigrist et al., 2002; Sigrist et al., 2003). Moreover, recently in vivo imaging of fluorescently labeled GluRIIA during synapse formation of larval NMJs over extended periods was established. Small PSDs grew by a nearly irreversible incorporation of GluRIIA from diffuse pools, suggesting a rate limiting role of this incorporation for PSD assembly (Rasse et al., 2005). GluRIIB, in contrast, was not visualized in vivo so far. For this purpose, EGFP (Clontech, Mountain View, CA) was cloned in the middle of the intracellular C-terminus (see 3.1.2.1 and 4.2.2; after amino acid T897, homologous position as used for GluRIIA) (Rasse et al., 2005) and this GFP fusion (g/uRIIB ${ }^{G F P}$ ) was expressed from a genomic gluRIIB clone. gluRIIB ${ }^{G F P}$ was able to rescue the otherwise embryonic lethal gluRIIA $A^{\text {null } I I B^{\text {null }} \text { mutant }}$ as efficient as the untagged wild type genomic construct gluRIIB $\left(I I B, 50 \%\right.$, IIB ${ }^{G F P}$, $55 \%$ of expected Mendelian ratio). 


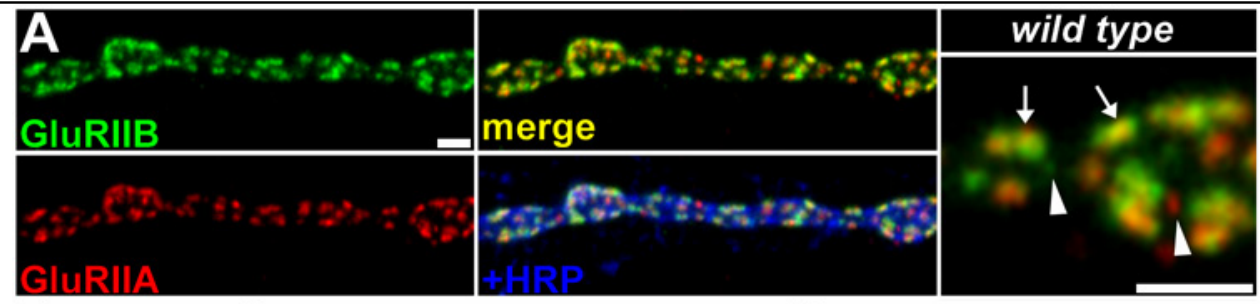

B

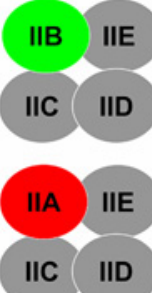

IIB GFP
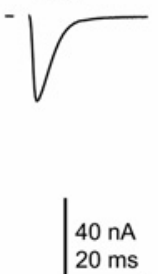

D
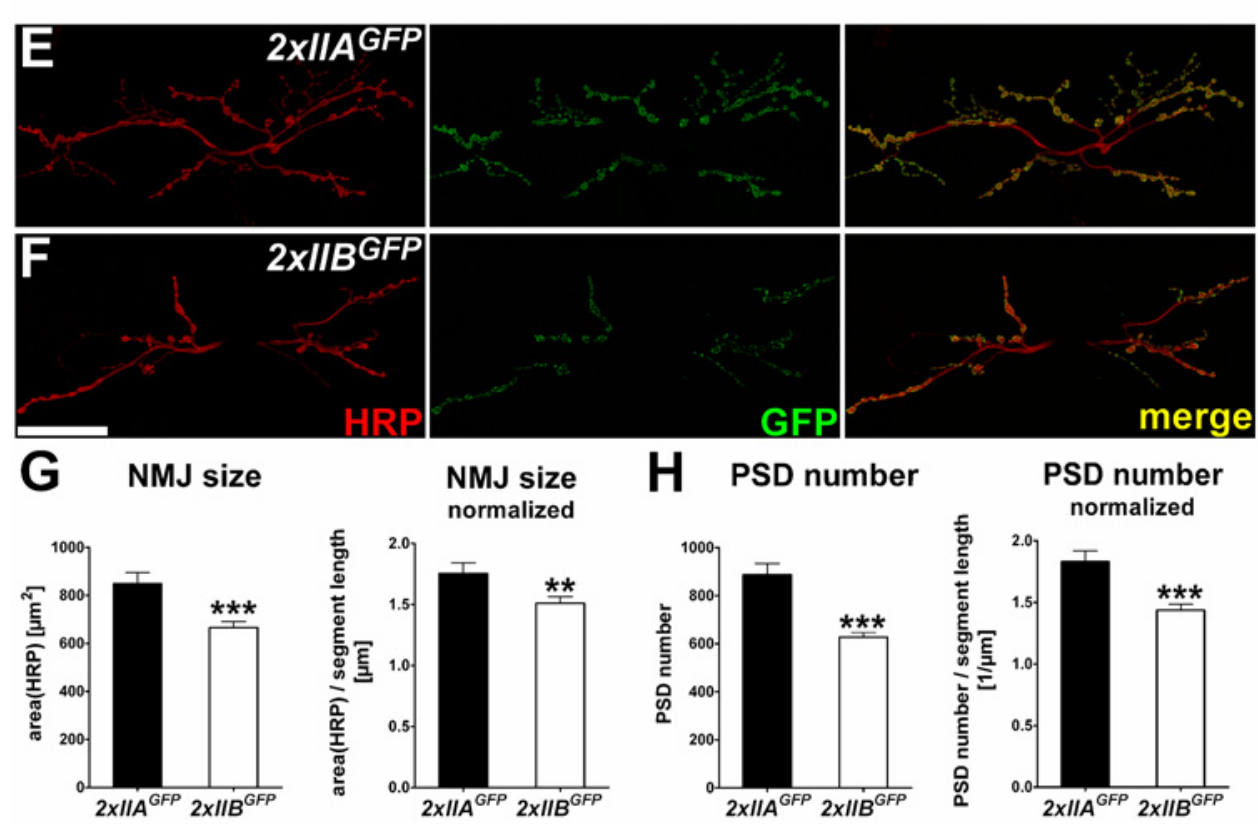
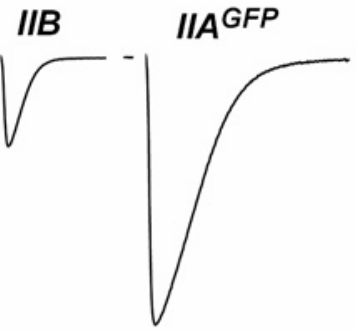

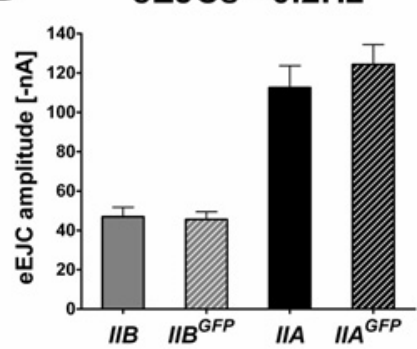

Fig. 34. Analysis of GFP-tagged GluRIIA and GluRIIB

$\boldsymbol{A}$ and $\boldsymbol{B}$, Two different NMJ-specific glutamate receptor complexes, incorporating GluRIIC, GluRIID, GluRIIE together with either GluRIIA (red) or GluRIIB (green), co-exist (B) but are not fully overlapping at single PSDs. Small, nascent PSDs (arrowheads in $\boldsymbol{A}$ ) show a heterogeneous receptor composition, while mature size PSDs (arrows in $\boldsymbol{A}$ ) show a rather balanced level of both receptor types. The NMJ morphology ( $\boldsymbol{A}$, muscle 4 ) is visualized by anti-HRP staining (blue). $\boldsymbol{C}$ and $\boldsymbol{D}$, GFP fusions of GluRIIA $\left(I I A^{G F P}\right.$, GFP fusion after AA893) (Rasse et al., 2005) and GluRIIB (IIB ${ }^{G F P}$, GFP fusion after AA897) and as controls unlabeled genomic transgenes of GluRIIA (IIA) and GluRIIB (IIB) were expressed to rescue

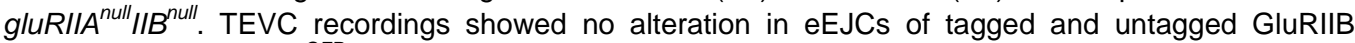
complexes. IIA and IIA ${ }^{G F P}$ currents, which were as well not distinguishable (Rasse 2005), are depicted for comparison. Shown are average eEJCs traces $(\boldsymbol{C})$ following $0.2 \mathrm{~Hz}$ stimulation. The mean eEJCs $(\boldsymbol{C})$ were: $I / B, 46.9 \pm 4.8 \mathrm{nA}, \mathrm{n}=10,\left\|B^{G F P}, 45.5 \pm 3.9 \mathrm{nA}, \mathrm{n}=10, \mathrm{p}=0.97 ;\right\| A, 112.6 \pm 11.1 \mathrm{nA}, \mathrm{n}=9, \| A^{G F P}$, 124.3 $\pm 10.0 n A, n=9, p=0.44$ (Rasse et al., 2005). For all pairs, currents of $I I B$ and $I / B^{G F P}$ were significantly smaller than IIA and IIA ${ }^{G F P} E J C s, p<0.001$. $\boldsymbol{E}$ and $\boldsymbol{F}$, Shown are immunostainings for HRP (red) and GFP (green) of $3^{\text {rd }}$ instar larvae (NMJ of muscles 6 and 7) expressing two copies of either gluRIIA ${ }^{\text {GFP }}\left(2 x I I A^{G F P}\right)$ or gluRIIB ${ }^{\text {GFP }}\left(2 x I I B^{G F P}\right)$ in the gluRIIA $A^{\text {null }} / B^{\text {null }}$ background. $\boldsymbol{G}$ and $\boldsymbol{H}$, Quantification of $\boldsymbol{E}$ and $\boldsymbol{F}$. Both the absolute NMJ size measured by the HRP area ( $\boldsymbol{G}$, left panel) and the PSD number $\left(\boldsymbol{H}\right.$, left panel) were significantly reduced at $2 x I I B^{G F P}$ NMJs when compared to $2 x I I A^{G F P}$. The significance also persisted after normalization to the respective segment length (see 7.5) of the larvae (G and $\boldsymbol{H}$, right panels). For values see text. Scale bars: $A, 2 \mu \mathrm{m} ; \mathrm{F}, 50 \mu \mathrm{m} .{ }^{\star \star} \mathrm{p} \leq 0.01,{ }^{\star \star \star} p \leq 0.001$. 
Next, the NMJ physiology of $3^{\text {rd }}$ instar larvae expressing either unlabeled or GFP-

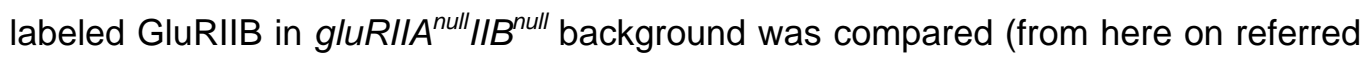
to as $I I B$ or $I I B^{G F P}$ ). Both $\mathrm{mEJCs}$ and eEJCs as measures of individual synaptic and overall $\mathrm{NMJ}$ transmission strength, respectively, were identical between $I / B$ and IIB ${ }^{G F P}$ (Fig. 34C,D) (mEJCs: IIB, 0.71 $\pm 0.04 n A, n=10, I I B^{G F P}, 0.66 \pm 0.03 n A, n=9$, $\mathrm{p}=0.50$; eEJCs: $\left.I I B, 46.9 \pm 4.8 \mathrm{nA}, \mathrm{n}=10, \quad I / B^{G F P}, 45.5 \pm 3.9 \mathrm{nA}, \mathrm{n}=10, \mathrm{p}=0.97\right)$. In contrast, as previously shown (Rasse et al., 2005) both IIA and IIA ${ }^{\text {GFP }}$ (rescue of

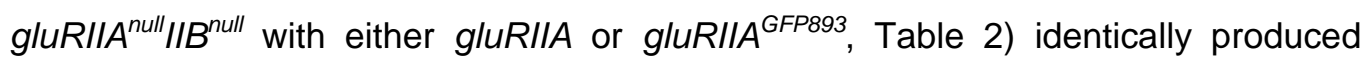
much higher eEJCs (Fig. 34C,D). Thus, it can be concluded that on the one hand GFP-tagging does not measurably affect the specific features of the subunits, and on the other hand that the specific differences between GluRIIA and GluRIIB for promoting NMJ transmission strength are well conserved also for the GFP-labeled subunits (being expressed in physiological levels from genomic constructs). Thus the GFP-labeled constructs were used throughout the further study.

As outlined above, favoring GluRIIA over GluRIIB expression increases the morphological size together with the number of synapses forming per NMJ (Reiff et al., 2002; Sigrist et al., 2002; Sigrist et al., 2003). To test whether these differences were also conserved for GFP-labeled GluRIIA or GluRIIB, gluRIIA ${ }^{\text {GFP }}$ or gluRIIB ${ }^{\text {GFP }}$ were expressed in the gluRIIA $A^{\text {null } I I B^{\text {null }}}$ background (2 genomic copies used, thus $2 x I I A^{G F P}$ and $2 x / I B^{G F P}$, respectively). In fact, NMJs of $2 x I I A^{G F P}$ late $3^{\text {rd }}$ instar larvae (Fig. 34E) were considerably larger than $2 x / I B^{G F P} \mathrm{NMJs}$ (Fig. 34F) (HRP signal) before and after animal size normalization (Fig. 34G, $\mathrm{H}$; see 3.3.5.4 and 7.5) (area(HRP) - 2xIIA ${ }^{G F P}, 849 \pm 46 \mu \mathrm{m}^{2}, \mathrm{n}=20,2 x \| B^{G F P}, 666 \pm 25 \mu \mathrm{m}^{2}, \mathrm{n}=20, \mathrm{p}<0.001$; area(HRP), normalized $-2 x I I A^{G F P}, 1.76 \pm 0.09 \mu \mathrm{m}, \mathrm{n}=20,2 x I I B^{G F P}, 1.51 \pm 0.05 \mu \mathrm{m}$, $\mathrm{n}=20, \mathrm{p}=0.0043$ ). Concomitantly, $2 x / I A^{G F P} \mathrm{NMJ}$ on average comprised about $30 \%$ more PSDs visualized by GFP immunostainings (PSD number - $2 x \| A^{G F P}, 888 \pm 46$, $\mathrm{n}=20,2 x \| B^{G F P}, 628 \pm 19, \mathrm{n}=20, \mathrm{p}<0.001 ;$ PSD number, normalized - $2 x \| A^{G F P}$, $1.83 \pm 0.09$ per $\mu \mathrm{m}, \mathrm{n}=20,2 x \|^{\mathrm{G}}{ }^{\mathrm{F} P}, 1.44 \pm 0.05$ per $\left.\mu \mathrm{m}, \mathrm{n}=20, \mathrm{p}<0.001\right)$.

In summary, the physiological properties of GFP-labeled constructs could not be discriminated from their unlabeled counterparts, while the specific differences in promoting or inhibiting NMJ synapse number and transmission strength appeared well conserved when using the GFP-tagged versions. Thus, an analysis to study the above mentioned differential role of the two receptor subtypes in PSD formation and $\mathrm{NMJ}$ development using these functional GFP fusions could be set-up. 


\subsubsection{Simultaneous in vivo imaging of GluRIIA and GluRIIB}

To allow the parallel in vivo imaging (see 3.3.2) of both receptor complexes expressed at the Drosophila NMJ, also equally functional fusions of GluRIIA (Rasse et al., 2005) and GluRIIB (Fig. 35C) with monomeric red fluorescent protein (mRFP) (Campbell et al., 2002) were used. Differentially tagged GluRIIA and GluRIIB were co-imaged at early $3^{\text {rd }}$ instar larval NMJs (of the ventral-acute muscle 27, see Fig. 9; type $\mathrm{lb}$ terminals) to follow the formation and maturation of individual PSDs. The analysis of the relative intensities of both GluRIIB ${ }^{\text {GFP }}$ and GluRIIA ${ }^{\text {mRFP }}$ (expressed from genomic transgenes in the gluRIIA$A^{\text {null }} / I B^{\text {null }}$ background, $I I B^{G F P} \& / I A^{\text {mRFP }}$, Table 2) at single PSDs allowed a quantitative specification of the PSD subunit composition, indicated by the ratio $r\left(r=I n t_{\text {rel }}\left(I I B^{G F P}\right) / I n t_{\text {rel }}\left(I I A^{\text {mRFP }}\right)\right.$; an exemplary ratio evaluation is shown in Fig. 19). In doing so, vast differences between the PSDs of IIB ${ }^{\text {GFP }}$ \&IIA $A^{\text {mRFP }}$ NMJs became obvious. Small PSDs showed large differences in the ratio $r$, means in the receptor content, which converged with increased PSD size (Fig. 35A). A similar divergence in the ratio $r$ was not observed in identically processed images of $\left\|A^{G F P} \&\right\| A^{\text {mRFP }}$ (Fig. 35B) and $\left\|B^{G F P} \&\right\| B^{\text {mRFP }}$ (Fig. 35C) NMJs (gluRIIA $A^{\text {null } I I B^{\text {null }}}$

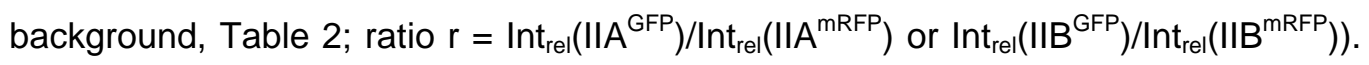
Accordingly, in both cases the respective GFP- and mRFP-labeled subunits almost completely overlapped at individual PSDs (Fig. 35B,C). This became also obvious when the ratio frequencies, subdivided into three ratio classes, covering GFP-rich (ratio $r>1.2$ ), mRFP-rich (ratio $r<0.8$ ) and balanced $(0.8<r<1.2)$ PSDs, were analyzed. While for $I I B^{G F P} \& \| A^{m R F P}$ all three PSD classes were represented with at least $20 \%$, more than $80 \%$ of $I I A^{\text {GFP }} \& / I A^{\text {mRFP }}$ and IIB ${ }^{\text {GFP }} \& / I B^{\text {mRFP }}$ PSDs were assigned to the middle ratio class (Fig. 35D). Thus, it can be concluded that the ratio differences observed after co-imaging of GluRIIA and GluRIIB are, at least to the largest extent, due to the distinct nature of GluRIIA and GluRIIB complexes.

To study the dynamic involvement of both receptor subtypes in PSD formation and maturation, identified NMJs of early $3^{\text {rd }}$ instar larvae expressing both GluRIIB ${ }^{\text {GFP }}$ and GluRIIA $^{\text {mRFP }}$ were subjected to repeated in vivo imaging (12h time intervals, rearing temperature $25^{\circ} \mathrm{C}$ ). In vivo imaging of GluRIIA had shown that new PSDs form de novo, often distant from the PSDs of pre-existing synapses (Rasse et al., 2005), and then grow until they reach a mature GluRIIA content. When GluRIIA ${ }^{\text {mFP }}$ and GluRIIB $^{\text {GFP }}$ were co-imaged, small, newly forming PSDs were typically rich of GluRIIA $^{\text {mRFP }}$ (Fig. 35E, octothorpes). In contrast, small GluRIIB ${ }^{\text {GFP }}$-rich PSDs were generally rather rare and frequently found in-between boutons (Fig. 35E,F, asterisks). As a strict definition of bouton and inter-bouton PSDs was not feasible, for analysis all PSDs were pooled independent of their localization at the NMJ. 

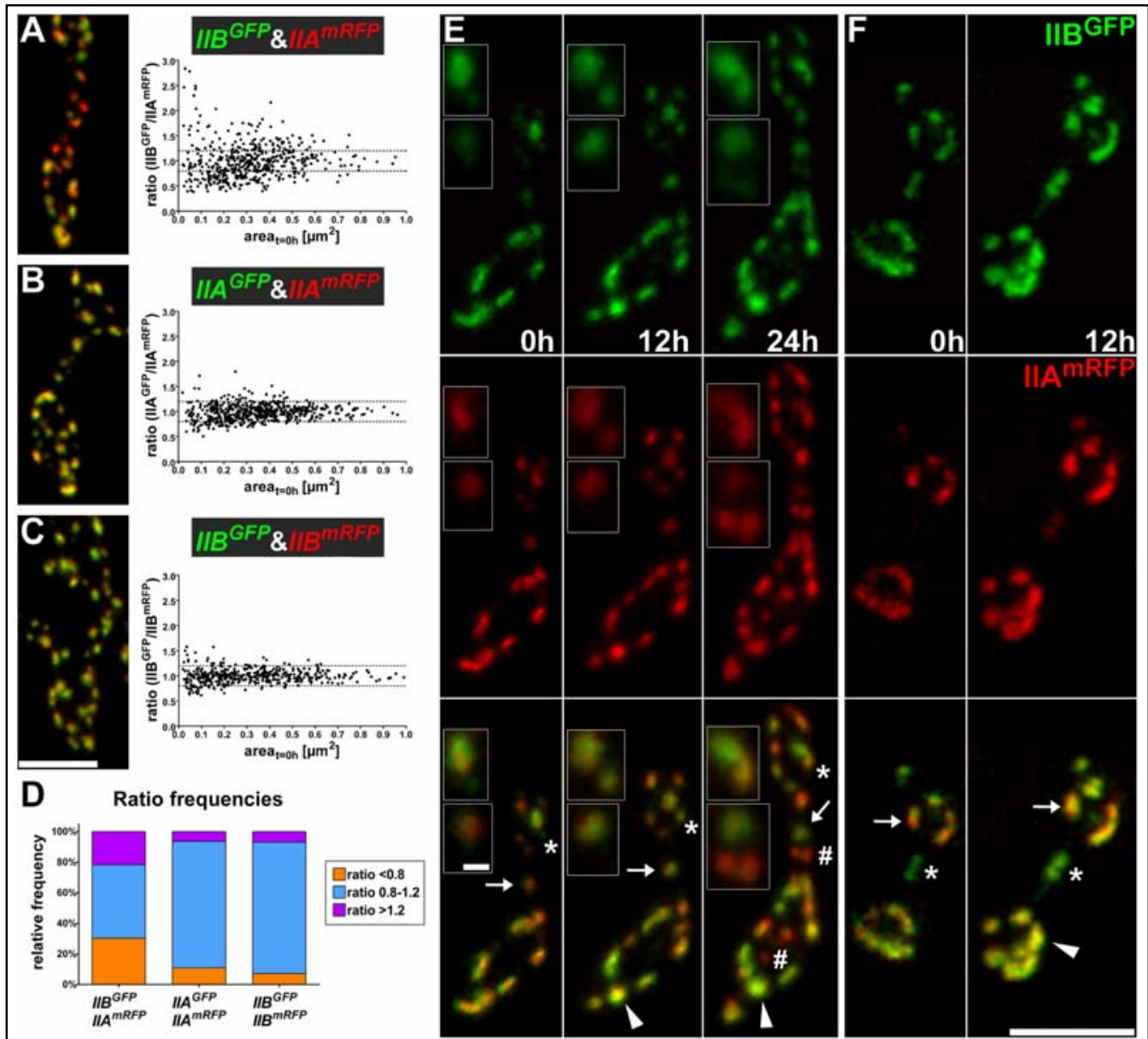

D

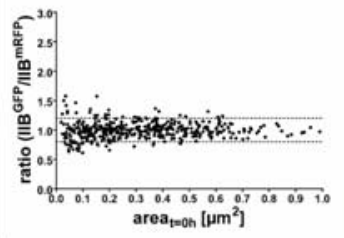

area $a_{t \rightarrow 0 h}\left[\mu m^{2}\right]$

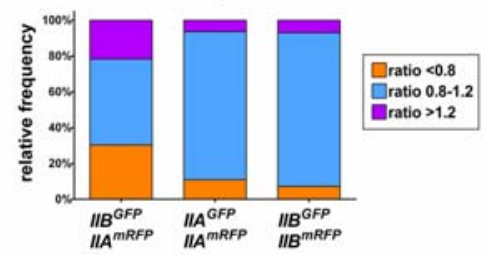

\section{Fig. 35. Size-dependent divergence of PSD subunit composition.}

$A$, In vivo imaging of $3^{\text {rd }}$ instar larvae expressing gluRIIB GFP and gluRIIA ${ }^{m R F P}$ in the lethal gluRIIA ${ }^{\text {null }} \| B^{\text {null }}$ background $\left(I I B^{G F P} \& \| A^{\text {mRFP }}\right)\left(12 \mathrm{~h}\right.$ time window; at $\left.25^{\circ} \mathrm{C}\right)$. Single $\left\|B^{G F P} \&\right\| A^{\text {mRFP }}$ PSDs (image, left panel) showed differential levels of GluRIIB ${ }^{\text {GFP }}$ (green) and GluRIIA ${ }^{\text {mRFP }}$ (red). A quantification of the relative intensities of both receptor types allowed quoting the ratio of GluRIIB $^{\text {GFP }}$ to GluRIIA ${ }^{\text {mRFP }}$ at individual PSDs [ratio $r=$ Int $_{\text {rel }}\left({ }^{\prime I B}{ }^{\text {GFP }}\right) / I_{\text {ntrel }}\left(I I A^{\text {mRFP }}\right)$ ]. Plotting the ratio $r$ over the initial PSD area (graph, right panel) uncovered a strong divergence in subunit composition for small PSDs and a convergence in the ratio $r$ with growing PSD size. $\boldsymbol{B}$ and $\boldsymbol{C}$, Analogue procedure as in $\boldsymbol{A}$. Using either gluRIIA $A^{G F P}$ and gluRIIA ${ }^{m R F P}$ or gluRIIB GFP and

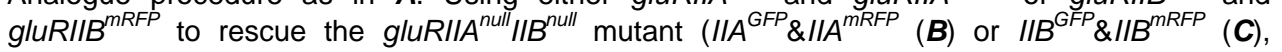
respectively) resulted, as expected, in a complete overlap of GFP and mRFP fluorescence (images, left panels). Here, a divergence in subunit composition [ratio $r=$ Intrel(IIX $\left.{ }^{G F P}\right) / /$ Int $_{\text {rel }}\left(\right.$ IIX $\left.{ }^{\text {mRFP }}\right) ; X$ represents $A$ or B] could not be observed (graphs, right panels). $D$, Quantification of the observed subunit ratios from $\boldsymbol{A}-\boldsymbol{C}$ subdivided into ratio classes $(r<0.8$; $0.8<r<1.2 ; r>1.2$; boundaries are indicated in the respective graphs of panels $\boldsymbol{A}-\boldsymbol{C}$.) While the vast majority of $I I A^{G F P} \& I I A^{m R F P}$ and $I I B^{G F P} \& / I B^{m R F P}$ PSDs showed balanced ratios, IIB ${ }^{G F P} \& \| A^{\text {mRFP }}$ PSDs could be clearly classified into three representative classes. The relative ratio frequencies of the particular classes were: $I I B^{G F P} \& \| A^{m R F P},<0.8-30.3 \%, 0.8-1.2-48.1 \%,>1.2-21.6 \%$, $\mathrm{n}=505,8 \mathrm{NMJs} ;\left\|A^{G F P} \&\right\| A^{\text {mRFP }},<0.8-10.9 \%, 0.8-1.2-82.8 \%,>1.2-6.3 \%, \mathrm{n}=559,8 \mathrm{NMJs}$; $\left\|B^{G F P} \&\right\| B^{m R F P},<0.8-7.2 \%, 0.8-1.2-85.9 \%,>1.2-6.9 \%, n=433,9$ NMJs. $\boldsymbol{E}$ and $\boldsymbol{F}$, In vivo imaging of $I I B^{G F P} \& I I A^{m R F P}$ early $3^{\text {rd }}$ instar larvae during $24 \mathrm{~h}(E)$ and $12 \mathrm{~h}(\boldsymbol{F})$ of NMJ development. Among small, nascent PSDs, GluRIIB ${ }^{\text {GFP }}$ rich PSDs (green) could be rarely observed (asterisks) while GluRIIA $^{\text {mRFP }}$ dominated PSDs (red) were particularly frequent (octothorpes). Both types showed further outgrowth and tended to equalize their differential receptor content (arrow). Mature size PSDs were characterized by a rather balanced receptor level (arrowheads). All

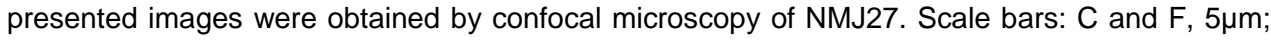
E, enlarged sections, $0.5 \mu \mathrm{m}$. 


\subsubsection{Receptor composition balances in the course of PSD growth and maturation}

So far, it was shown that newly forming synapses are heterogeneous in glutamate receptor composition, typically being dominated by GluRIIA ${ }^{\text {mRFP }}$. Throughout their further outgrowth, however, such newly forming, GluRIIA ${ }^{\text {mRFP }}$-rich PSDs (arrows in Fig. 35,F) seemed to become more balanced in receptor composition at later observation time points. Vice versa, small PSDs initially rich of GluRIIB ${ }^{\text {GFP }}$ later appeared more balanced as well (Fig. 35E,F, asterisks).

To quantify this behavior, 505 individual PSDs (from 8 NMJs, muscle 27) identified at $\mathrm{t}=0 \mathrm{~h}$ and re-identified at $\mathrm{t}=12 \mathrm{~h}$ were analyzed. As expected, the PSD population (example PSDs shown in Fig. 37A) substantially increased its average size (resulting from a maximum overlay of both GluRIIA ${ }^{\text {mRFP }}$ and GluRIIB ${ }^{\text {GFP }}$ ) from $0.323 \pm 0.08 \mu m^{2}$ to $0.414 \pm 0.09 \mu \mathrm{m}^{2}, \mathrm{p}<0.001$ (Fig. 36A). Thereby, an inverse relation between the initial PSD size and the further growth could be observed (Fig. 36B). Moreover, the development of the receptor subunit composition of single PSDs, numerically quoted by the intensity ratio $r\left(\operatorname{Int}_{\text {rel }}\left(I I B^{G F P}\right) / / \operatorname{lnt}_{\text {rel }}\left(I I A^{\text {mRFP }}\right)\right)$, was followed. Nine PSD classes, discriminated by the initial size (small, $<0.2 \mu \mathrm{m}^{2}$; medium, 0.2-0.4 $\mathrm{m}^{2}$; large, $\left.>0.4 \mu \mathrm{m}^{2}\right)$ and ratio $r(<0.8,0.8-1.2,>1.2)$, were established (absolute numbers, see Fig. 37B; proportion, see Fig. 37D, tables). As mentioned above, among the small PSDs observed at the first time point, GluRIIA ${ }^{\text {mRFP }}$-rich ones $(r<0.8)$ were most prominent while the majority of medium and large PSDs showed a balanced receptor content $(0.8 \leq r \leq 1.2)$. Fig. $37 \mathrm{C}$ demonstrates that the change of the ratio $r$ during the observation interval of $12 \mathrm{~h}\left(\Delta \mathrm{r}_{\Delta \mathrm{t}=12 \mathrm{~h}}\right)$ was reciprocally related to the start ratio $\mathrm{r}\left(\mathrm{r}_{\mathrm{t}=0 \mathrm{~h}}\right)$. In other words, the PSD population showed a strong trend to balance the receptor composition over time.

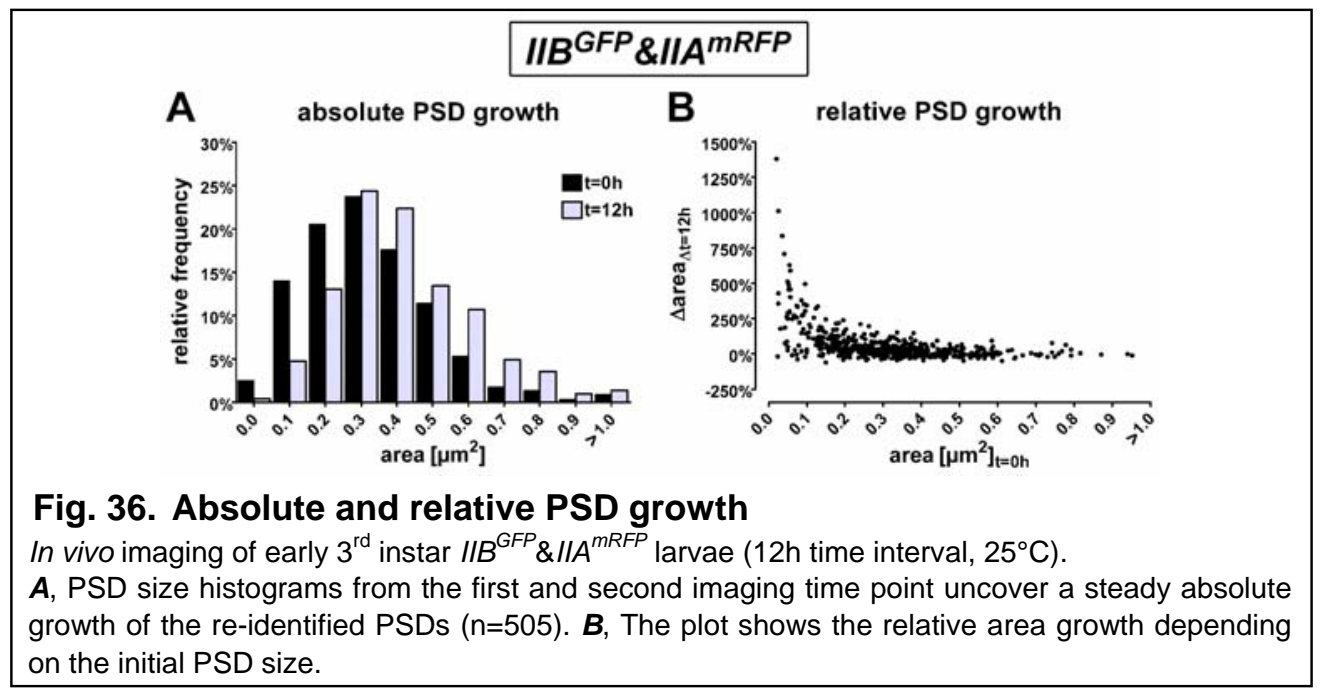


The detailed analysis of the particular size classes showed that small PSDs rich of GluRIIA $^{\text {mRFP }}$ (Fig. 37D, $r<0.8$ ) specifically increased their GluRIIB ${ }^{\text {GFP }}$ intensity. Vice versa, small GluRIIB ${ }^{\text {GFP }}$-rich PSDs (Fig. 37D, r>1.2) specifically increased their GluRIIA $^{\text {mRFP }}$ levels. PSDs harboring both receptor subtypes to a similar extent, on average maintained steady relative intensities (Fig. 37D, $0.8 \leq r \leq 1.2$ ). Thus, all subpopulations either converged to or kept a balanced receptor composition.

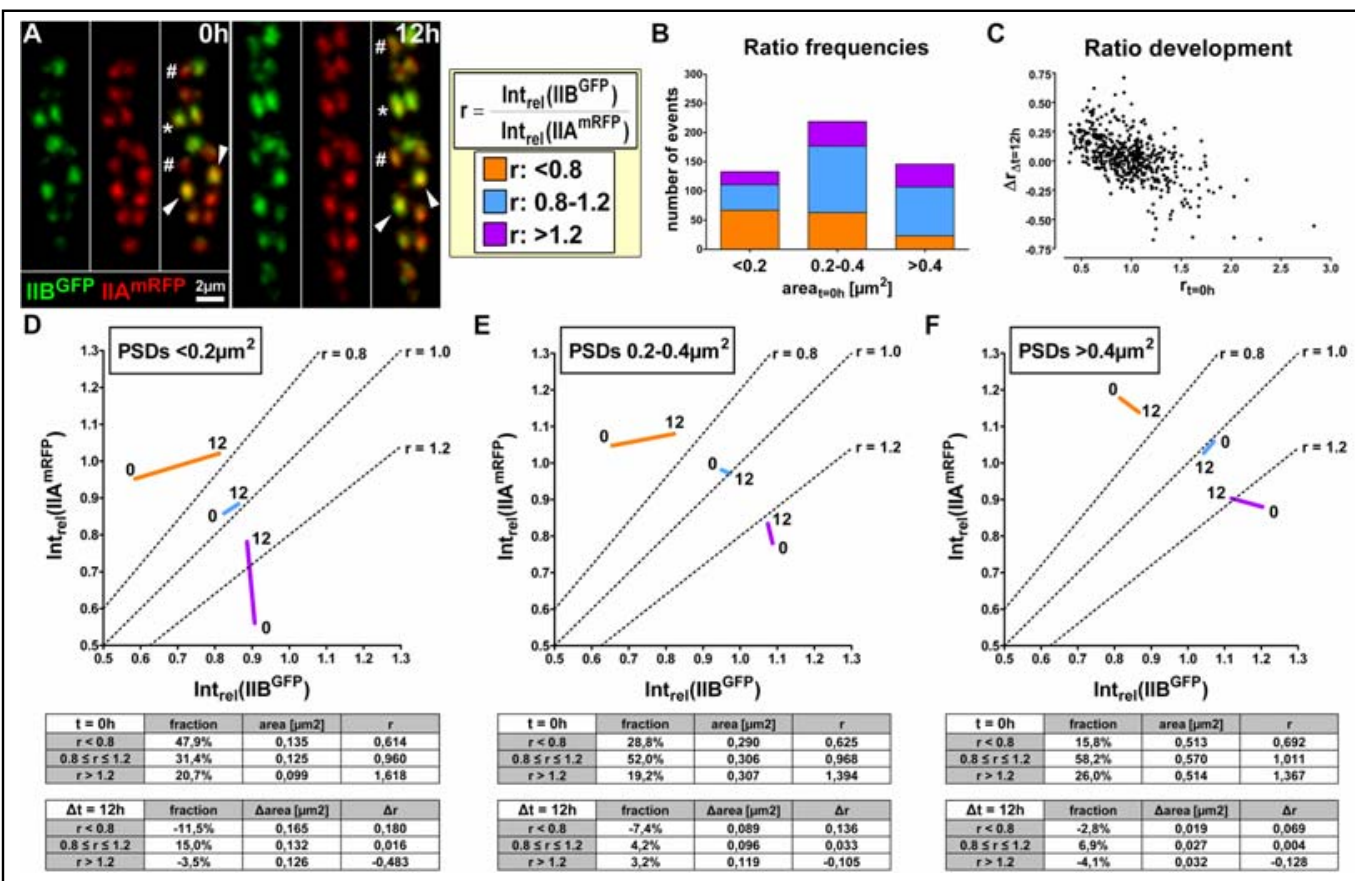

Fig. 37. PSD subunit composition development depends on initial stage

$\boldsymbol{A}$, Early $3^{\text {rd }}$ instar $I I B^{G F P} \& / I A^{\text {mRFP }}$ larvae were subjected to confocal in vivo imaging $\left(\right.$ at $\left.25^{\circ} \mathrm{C}\right)$. Single PSDs were imaged and re-identified after an experimental time window of $12 \mathrm{~h}$. Small PSDs rich of GluRIIA ${ }^{\text {mRFP }}$ (red) tended to specifically integrate GluRIIB ${ }^{\text {GFP }}$ containing complexes (green) and vice versa (octothorpes and asterisks, respectively), while both PSD types grew substantially in size. Large PSDs rather maintained a stable net receptor content and barely increased their size (arrowheads). $\boldsymbol{B}$, Quantification of the absolute ratio frequencies depending on the initial PSD size. While small PSDs were largely rich of GluRIIA ${ }^{\text {mRFP }}(r>0.8)$, large PSDs mainly showed a balanced subunit composition $(0.8<r<1.2)$. $C$, Reciprocal relation of the absolute change in the ratio $r_{\Delta t=12 h}$ and the initial ratio $r_{t=0 h}(n=505)$. $\boldsymbol{D}-\boldsymbol{F}$, Development of the mean relative intensities of GluRIIA ${ }^{\text {mRFP }}$ and GluRIIB $^{\text {GFP }}$ classified by the initial PSD size and ratio $r$ (for absolute frequencies see panel $\boldsymbol{B}$ ). Small PSDs $\left(<0.2 \mu \mathrm{m}^{2}, \boldsymbol{D}\right)$ dominated by GluRIIA $^{\text {mRFP }}(r<0.8)$ mainly integrated GluRIIB ${ }^{\text {GFP }}$ complexes during further development $(12 h)$, while PSDs rich of GluRIIB ${ }^{\text {GFP }}(r>1.2)$, which were significantly smaller $(p<0.05)$, specifically incorporated GluRIIA ${ }^{\text {mRFP }}$. PSDs with a balanced receptor content $(0.8<r<1.2)$ maintained their state. All three PSD types approximately doubled their size in the $12 \mathrm{~h}$ observation interval. Medium size PSDs $\left(0.2-0.4 \mu \mathrm{m}^{2}, E\right)$ increased their size in the average about $30 \%$, as well independent of the receptor composition. Their incorporation behavior was similar to small PSDs, even though less pronounced. Large PSDs $\left(>0.4 \mu \mathrm{m}^{2}, F\right)$ basically kept a stable size but nevertheless showed a convergence of the ratio $r$ to a balanced level. The mean parameters are given in the tables below the respective charts. The dashed lines indicate the ratios $0.8,1.0$ and 1.2 , respectively. Scale bar: $2 \mu \mathrm{m}$.

PSDs initially rich of GluRIIB ${ }^{\text {GFP }}(r>1.2)$ were significantly smaller than those PSDs containing notable levels of GluRIIA ${ }^{\text {mRFP }}$ (Fig. 37D, table, $p<0.05$ ). However, the growth of small PSDs ( $\triangle$ area) was not statistically different among the three ratio 
classes (Fig. 37D, table; p>0.1). Medium PSDs (Fig. 37E) still showed remarkable growth (about two thirds of the growth observed for small PSDs) apparently independent of the initial receptor composition. Similar to small PSDs, the receptor composition became more balanced. Large PSDs (Fig. 37F) experienced only minor changes in PSD size and in the intensities of the respective receptor subunits. However, the tendency to balance the levels of both receptor complexes remained.

To sum up, the initial diverse glutamate receptor subtype composition balanced in the course of the maturation of individual PSDs. This was obviously accomplished by the specific incorporation of either GluRIIA or GluRIIB complexes depending on the initial receptor content of the respective PSD.

\subsubsection{Distinct PSD incorporation behavior of GluRIIA and GluRIIB}

Previously, fluorescence recovery after photobleaching (FRAP) experiments showed that the incorporation of GluRIIA is maximal at growing PSDs but low at mature, no longer growing PSDs (Rasse et al., 2005). These experiments have been done in the presence of unlabeled GluRIIB. To directly compare the PSD incorporation behavior of GluRIIA to GluRIIB, both GluRIIB $^{\text {GFP }}$ and GluRIIA ${ }^{\text {mRFP }}$ (co-expressed in the

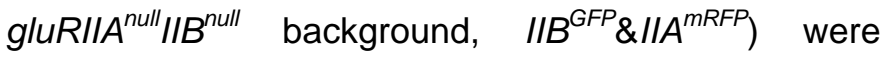
imaged, bleached and re-imaged after $24 \mathrm{~h}$ recovery (Fig. 38). As expected, GluRIIA ${ }^{\text {mRFP }}$ incorporation was strong at few synapses but very faint at the remaining synapses (Fig. 38, arrowhead and arrow, respectively). In clear contrast, GluRIIB ${ }^{\text {GFP }}$ showed a uniform recovery over all PSDs. In result, the overlay of the FRAP signals showed a large fraction of synapses with essentially only GluRIIB ${ }^{\mathrm{GFP}}$ incorporation, while some PSDs were dominated by strong GluRIIA ${ }^{\text {mRFP }}$ incorporation.

Fig. 38. Differential PSD incorporation behavior of GluRIIA and GluRIIB complexes

FRAP experiment of $\left\|B^{G F P} \&\right\| A^{\text {mRFP }}$ early $3^{\text {rd }}$ instar NMJs $(24 \mathrm{~h}$ recovery time; bleaching of both the GFP and mRFP channel). GluRIIA $^{\text {mRFP }}$ (red) and GluRIIB ${ }^{\text {GFP }}$ (green) containing complexes showed a differential incorporation behavior. While GluRIIB ${ }^{\text {GFP }}$ recovered rather equally over all PSDs, GluRIIA ${ }^{\text {mRFP }}$ showed strong FRAP at some PSDs (arrowhead) but almost no FRAP at the remaining PSDs (arrow). Scale bar: $5 \mu \mathrm{m}$.

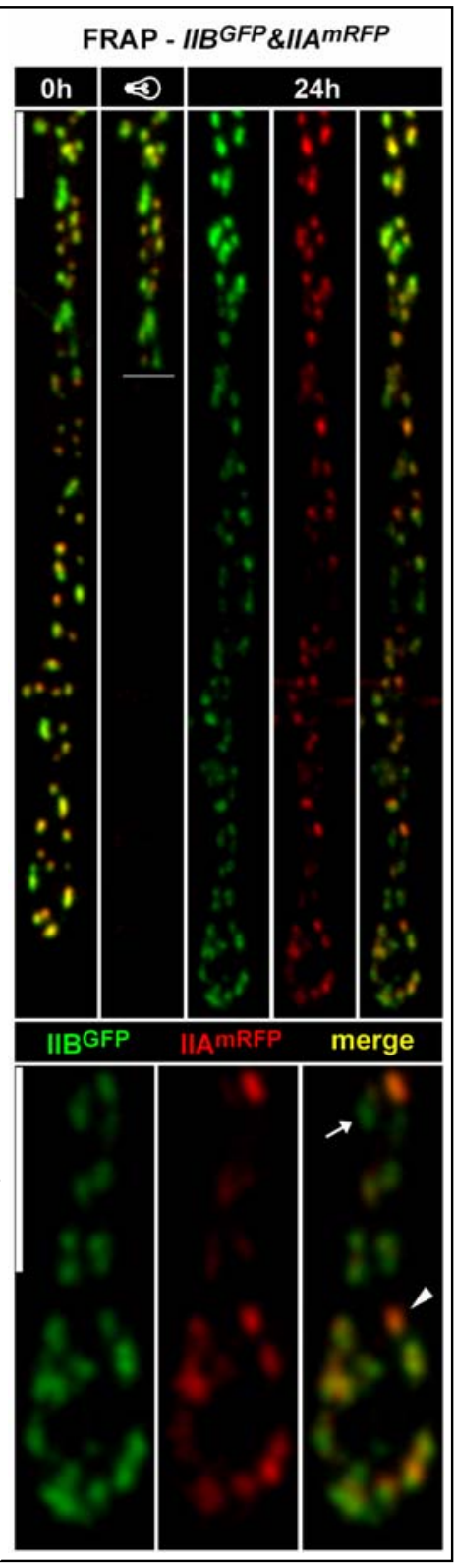


The choice of the fluorophore might have a principal effect on the FRAP behavior. Thus, GFP and mRFP were exchanged resulting in GluRIIA ${ }^{\text {GFP }}$ and GluRIIB ${ }^{\text {mRFP }}$

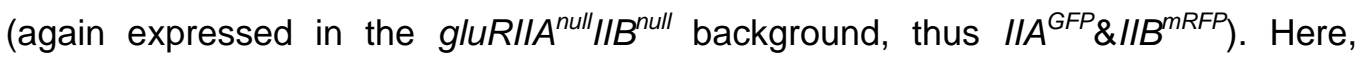
FRAP of GluRIIA ${ }^{\text {GFP }}$ was confined to few synapses (Fig. 40A) while FRAP of GluRIIB $^{\text {mRFP }}$ exhibited uniform incorporation over the before bleached PSDs. Thus, qualitatively, GluRIIA and GluRIIB FRAP were independent of fluorophore choice. As reported before, GluRIIA incorporation was restricted to few PSDs, previously shown to represent growing PSDs (Rasse et al., 2005). In clear contrast GluRIIB apparently incorporated equally into all PSDs, both growing and matured ones.

\subsubsection{The CTD of GluRIIA contributes to suppress GluRIIA incorporation at mature PSDs}

To consolidate the mere optical impression, the FRAP experiments were quantified. Thereto, the FRAP intensity of the formerly bleached PSDs was related to the mean intensity of the non-bleached PSDs $(\mathrm{t}=24 \mathrm{~h})$ and normalized to the initial time point $(\mathrm{t}=0 \mathrm{~h})$, thereby forming a recovery rate (see 3.3.5.3). In both FRAP experiments $\left(I I B^{G F P} \& I I A^{m R F P}\right.$, Fig. 38 and $I I A^{G F P} \& I I B^{m R F P}$, Fig. 40A), recovery rates of GluRIIA were very heterogeneous, with the majority of PSDs showing only little or strong recovery, not fitting a non-linear Gaussian regression (Fig. 39A and Fig. 39C, fit values: 0.912 and 0.890 , respectively). In contrast, in both cases the recovery of GluRIIB followed a Gaussian distribution (Fig. 39A and Fig. 39C, fit values: 0.978 and 0.979). While the observed recovery rates varied sparsely (potentially reflecting a faster assembly of mRFP than of GFP) the specific FRAP patterns were essentially unaffected by the fluorophore exchange.

GluRIIA and GluRIIB are structurally similar subunits (Petersen et al., 1997). Which structural elements might then encode this different targeting behavior of the two receptor subtypes? The intracellular C-terminal domains (CTDs) have been directly implicated into the subunit-specific targeting behavior of mammalian glutamate receptor (Barry and Ziff, 2002; Malinow and Malenka, 2002; Bredt and Nicoll, 2003). Hence, C-terminal chimeras of GluRIIA and GluRIIB, each labeled with GFP, were established $\left(A A B^{G F P}\right.$ and $B B A^{G F P}$ ). Both chimeras rescued the otherwise lethal gluR $\left\|A^{\text {null }}\right\| B^{\text {null }}$ background giving rise to adult flies. For FRAP experiments, genomic transgenes of $A A B^{G F P}$ together with GluRIIB ${ }^{\text {mRFP }}\left(A A B^{G F P} \& / I B^{m R F P}\right.$, Fig. 39B and Fig.

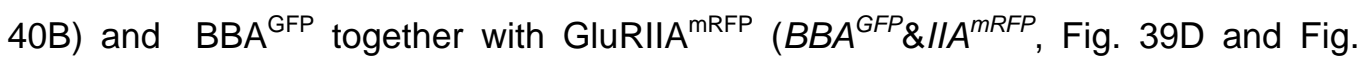
$40 C$ ) were expressed in the gluRIIA ${ }^{\text {null }} / I B^{\text {null }}$ background. The recovery of $A A B^{G F P}$ (Fig. 39B and Fig. 40B) was more Gaussian-like (fit value: 0.965) as observed before 
for GluRIIA ${ }^{\text {GFP }}$ (Fig. 39A), means more PSDs exhibiting moderate instead of faint FRAP could be detected.

Fig. 39. Quantification of the differential FRAP behavior

FRAP experiments of early $3^{\text {rd }}$ instar NMJs with $24 \mathrm{~h}$ recovery time after bleaching of both the GFP and mRFP channel. All transgenes were expressed in the gluR IIA ${ }^{\text {null }} \| B^{\text {null }}$ background.

$\boldsymbol{A}-\boldsymbol{E}$, Quantitative FRAP data of individual PSDs. $I I A^{G F P} \& / I B^{m R F P}(\boldsymbol{A})$ $\left(n=656,6\right.$ NMJs): GluRIIB ${ }^{\text {mRFP }}$ showed equal incorporation over all PSDS (Gaussian fit: 0.978) while GluRIIA GFP recovery was non-uniform (Gaussian fit: 0.912). $A A B^{G F P} \& I I B^{m R F P}(B)(n=326$, 6 NMJs): compared to unmodified GluRIIA $^{\text {GFP }}, A A B^{\text {GFP }}$ (GluRIIA with the GluRIIB $^{\text {GFP }}$ C-terminus) showed a rather Gaussian-like recovery pattern (Gaussian fit: 0.965). GluRIIB ${ }^{\text {mRFP }}$ again demonstrated uniform recovery (Gaussian fit: 0.992). IIB ${ }^{G F P} \& / I A^{m R F P}$ (C) $(\mathrm{n}=498,7 \mathrm{NMJs})$ : the fluorophore exchange did not alter the incorporation behavior with GluRIIB ${ }^{\text {GFP }}$ recovering consistently (Gaussian fit: 0.979) and GluRIIA ${ }^{\text {mRFP }}$ showing strongly deviating FRAP at individual PSDs (Gaussian fit: 0.890). $B B A^{G F P} \& I I A^{m R F P}(D)(\mathrm{n}=377,7 \mathrm{NMJs})$ : similar to GluRIIB ${ }^{\mathrm{GFP}}$ (C), BBA ${ }^{\mathrm{GFP}}$ (GluRIIB with the GluRIIA ${ }^{\text {GFP }}$ Cterminus) exhibited equal FRAP over all PSDs (Gaussian fit: 0.985) while GluRIIA $^{\text {mRFP }}$ kept on incorporating differently (Gaussian fit: 0.885). $\left\|B^{G F P} \&\right\| A^{m R F P}$ with a concomitant blockage of presynaptic activity through the mosaic expression of tetanus toxin in motoneurons (ok319tht $) \quad(E) \quad(\mathrm{n}=303,7$ NMJs $):$ in comparison to the similar situation without activity blockage (C), GluRIIB $^{\text {GFP }}$ recovery was strongly reduced and often even below the

A FRAP $-\left\|A^{G F P} \&\right\| B^{m R F P}$

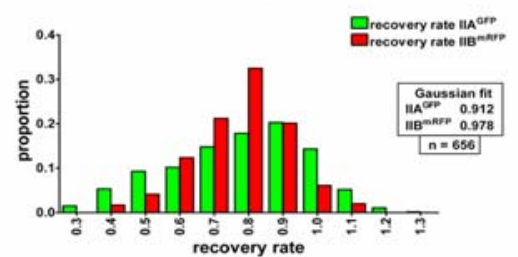

B FRAP - $A A B^{G F P} \& \| B^{m R F P}$

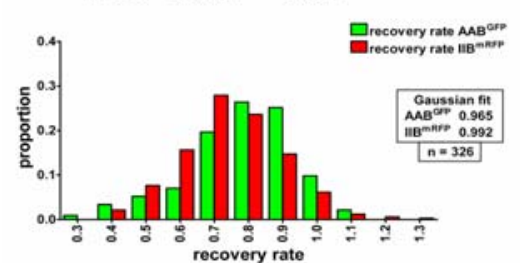

C FRAP $-\left\|B^{G F P} \&\right\|^{m R F P}$

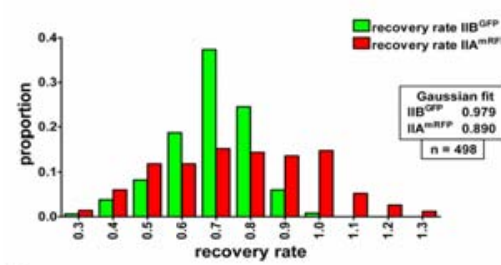

D FRAP - BBA $A^{G F P} \& \|^{m R F P}$

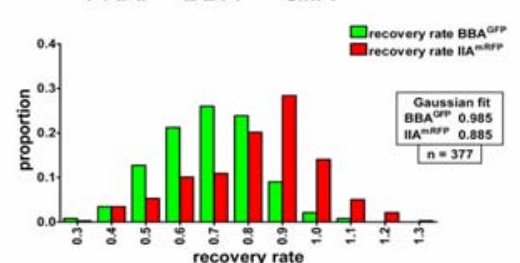

E FRAP - IIB ${ }^{G F P} \& A^{m R F P}$

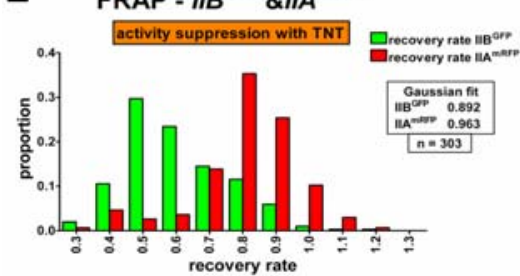

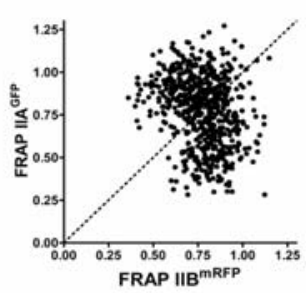
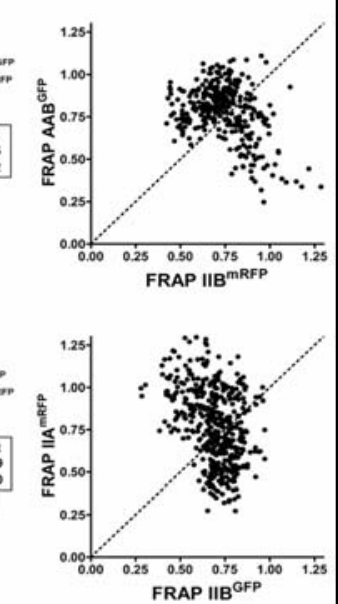
FRAP IIB ${ }^{\text {GFP }}$
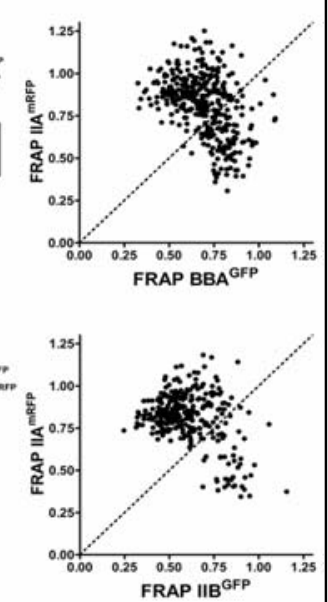
FRAP IIB ${ }^{\text {GFP }}$ detection limit, explaining the untypical Gaussian fit value of 0.892 . GluRIIA ${ }^{\text {mRFP }}$ showed the typical nonuniform pattern with an elevation of the overall recovery (Gaussian fit: 0.963). In cases, where both GluRIIB $^{\text {GFP }}$ and GluRIIA ${ }^{\text {mRFP }}$ FRAP were very weak, the recovery could not be determined. As ok319gal4 did not express at NMJ27, the analysis shown in $\boldsymbol{E}$ derived from NMJ 14 (see Fig. 45).

Thus, the CTD of GluRIIA seems to contribute to suppress GluRIIA incorporation into PSDs. The recent report that GluRIIA incorporation is restricted to growing PSDs (Rasse et al., 2005) implies that the CTD of GluRIIA is involved in shutting down GluRIIA incorporation at mature PSDs. Notably, the CTD of GluRIIA was not sufficient to confer GluRIIA-type incorporation behavior to BBA ${ }^{\text {GFP }}$ (Fig. 39E, 
compare to Fig. 39D). In fact, BBA ${ }^{\text {GFP }}$ still recovered uniformly (fit value: 0.985). Moreover, the FRAP pattern of GluRIIA ${ }^{\text {mRFP }}$ remained unaffected by the presence of BBA $^{\text {GFP }}$ (fit value: 0.885). In summary, the C-terminal domains of GluRIIA and

GluRIIB seem to contribute to their different PSD incorporation behavior.

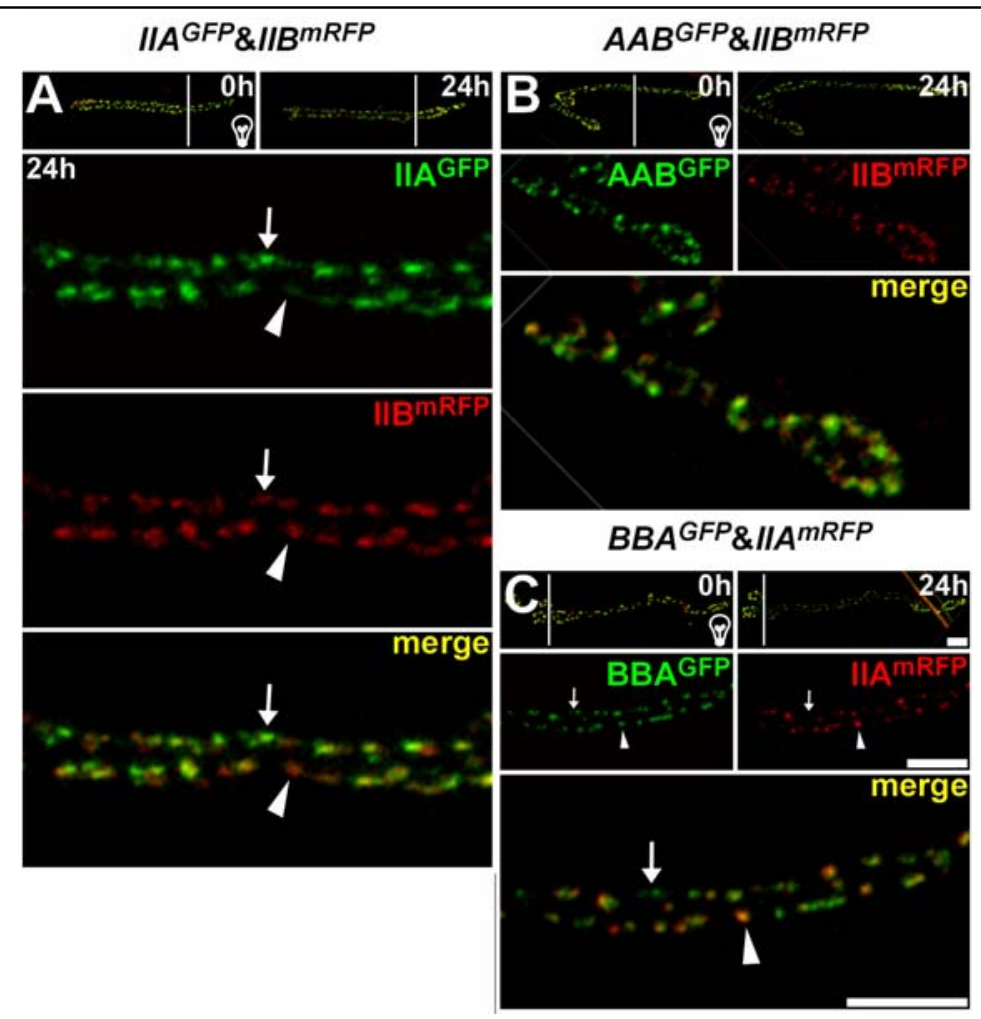

Fig. 40. The differential incorporation characteristics involve the receptor C-terminus - part I

Shown are confocal microscopy images derived from FRAP experiments on early $3^{\text {rd }}$ instar larvae (NMJ27), illustrating the initial (Oh), the bleached (indicated by the bulb) and the recovered state $(24 \mathrm{~h})$ of glutamate receptor levels at single PSDs. All transgenes were expressed in the gluRIIA $A^{\text {null }} / B^{\text {null }}$ background.

$\boldsymbol{A},\left\|A^{\text {GFP }} \&\right\| \mathrm{B}^{\text {mRFP }}$. GluRIIB ${ }^{\text {mRFP }}$ (green) showed equal FRAP over all PSDs while GluRIIA ${ }^{\text {GFP }}$ (red) only recovered substantially at some PSDs (compare arrows and arrowheads). $\boldsymbol{B}$, $A A B^{G F P} \& I I B^{m R F P}$. In comparison to GluRIIA ${ }^{\text {GFP }}(\boldsymbol{A}), A^{\text {AAB }}{ }^{\text {GFP }}$ (green, GluRIIA with the GluRIIB ${ }^{\text {GFP }}$ C-terminus) showed a more uniform recovery with PSDs exhibiting either strong or at least moderate FRAP. GluRIIB $^{\text {mRFP }}$ (red) again exhibited equal FRAP. $C, B B A^{G F P} \& I_{1} A^{\text {mRFP }}$. BBA ${ }^{\text {GFP }}$ (green, GluRIIB with the GluRIIA ${ }^{\text {GFP }}$ C-terminus) showed uniform FRAP over all PSDs while GluRIIA $^{\text {mRFP }}$ (red) incorporated non-uniformly (arrows and arrowheads). Scale bars: $5 \mu \mathrm{m}$.

\subsubsection{Distinct PSD incorporation of GluRIIA and IIB persists in the absence of the other complex}

To clarify whether the differentiation of incorporation behavior between GluRIIA and GluRIIB would be established only if both receptor complexes were present simultaneously, FRAP experiments in the respective absence of one complex subtype were performed. For this purpose, genomic transgenes of either GluRIIA ${ }^{\text {GFP }}$ 
and GluRIIA ${ }^{\text {mRFP }}\left(I I A^{\text {GFP }} \& I I A^{\text {mRFP }}\right)$ or GluRIIB ${ }^{\text {GFP }}$ and GluRIIB ${ }^{\text {mRFP }}$ (IIB ${ }^{\text {GFP }}$ \&IIB ${ }^{\text {mRFP }}$ ) were expressed in the gluR\|I$A^{\text {null }} \| B^{\text {null }}$ background.

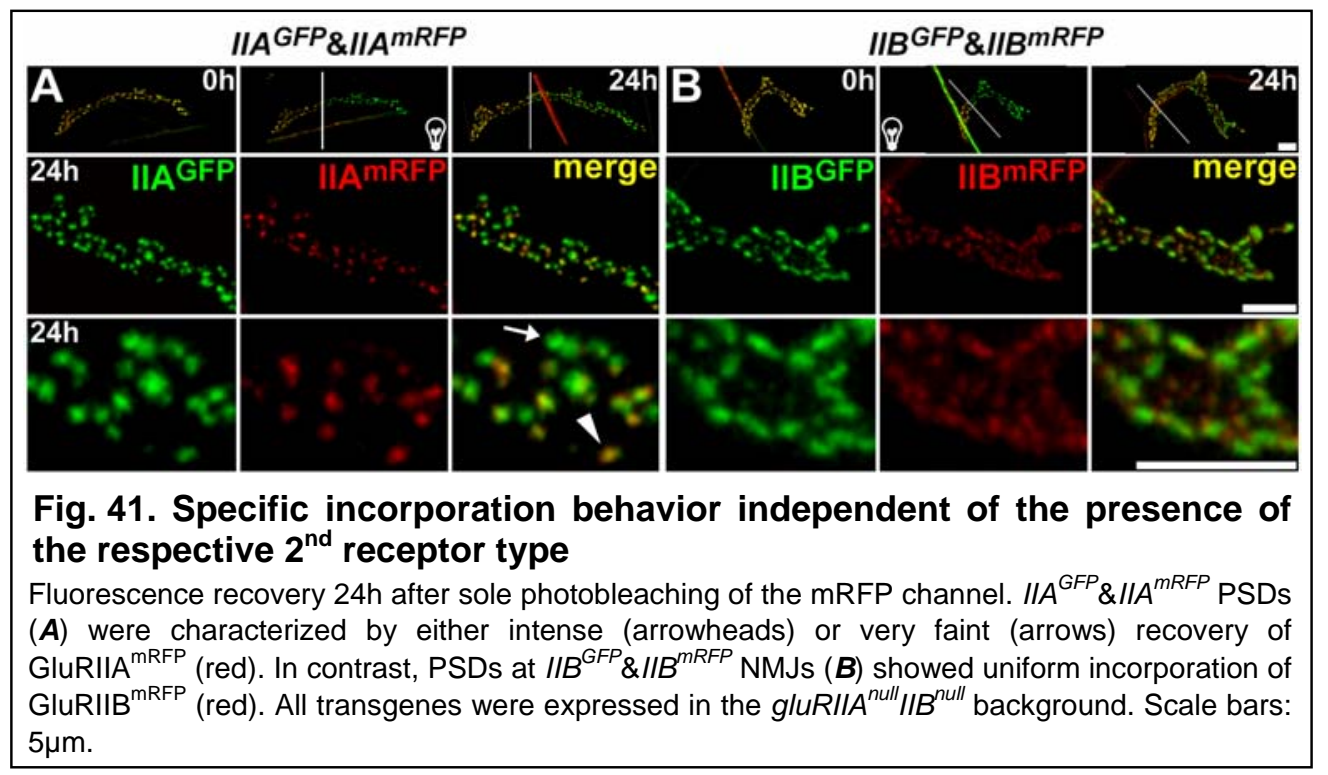

To score also potential PSDs showing no measurable recovery (after $24 \mathrm{~h}$ ), only the mRFP channel was bleached at $\mathrm{t}=0 \mathrm{~h}$. Interestingly, the non-uniform recovery pattern of GluRIIA complexes persisted in the absence of GluRIIB, with GluRIIA ${ }^{\text {mRFP }}$ showing strong recovery at some but only very faint recovery at the other PSDs (Fig. 41A, arrowhead and arrow, respectively), very similar as before seen in the presence of GluRIIB (Fig. 38 and Fig. 40A) (Rasse et al., 2005). In result, only a partial overlap between the recovered GluRIIA ${ }^{\text {mRFP }}$ and the non-bleached GluRIIA ${ }^{\text {GFP }}$ could be observed. PSDs lacking GluRIIA showed a uniform incorporation of GluRIIB ${ }^{\text {mRFP }}$ (Fig. 41B) that almost entirely overlapped with the non-bleached GluRIIB ${ }^{\text {GFP }}$. It can thus be concluded that the subunit specific incorporation properties persisted also when the respective glutamate receptor complex was absent.

This result allowed to evaluate the role of the CTDs in additional FRAP experiments. When $A A B^{\text {GFP }}$ and GluRIIA ${ }^{\text {mRFP }}\left(A A B^{\text {GFP }} \& / I A^{\text {mRFP }}\right.$, Fig. 42A) were subjected to FRAP (bleaching of both channels), $A A B^{\text {GFP }}$ showed a more uniform FRAP over all PSDs while GluRIIA $^{\text {mRFP }}$ kept its typical non-uniform recovery pattern. Thus, integration of $A A B^{G F P}$ into PSDs seemed to be favored over GluRIIA ${ }^{\text {mRFP }}$ incorporation, again consistent with the notion that the CTD of GluRIIA contributes to suppress GluRIIA incorporation. Similarly, FRAP of individual PSDs was compared between BBA ${ }^{\text {GFP }}$ and GluRIIB (BBA ${ }^{G F P} \& / I B^{m R F P}$, Fig. 42B). While both showed uniform FRAP, GluRIIB $^{\text {mRFP }}$ incorporation seemingly outcompeted $B B A^{G F P}$, suggesting that the CTD of GluRIIA tends to suppress PSD incorporation also in fusion to GluRIIB. 


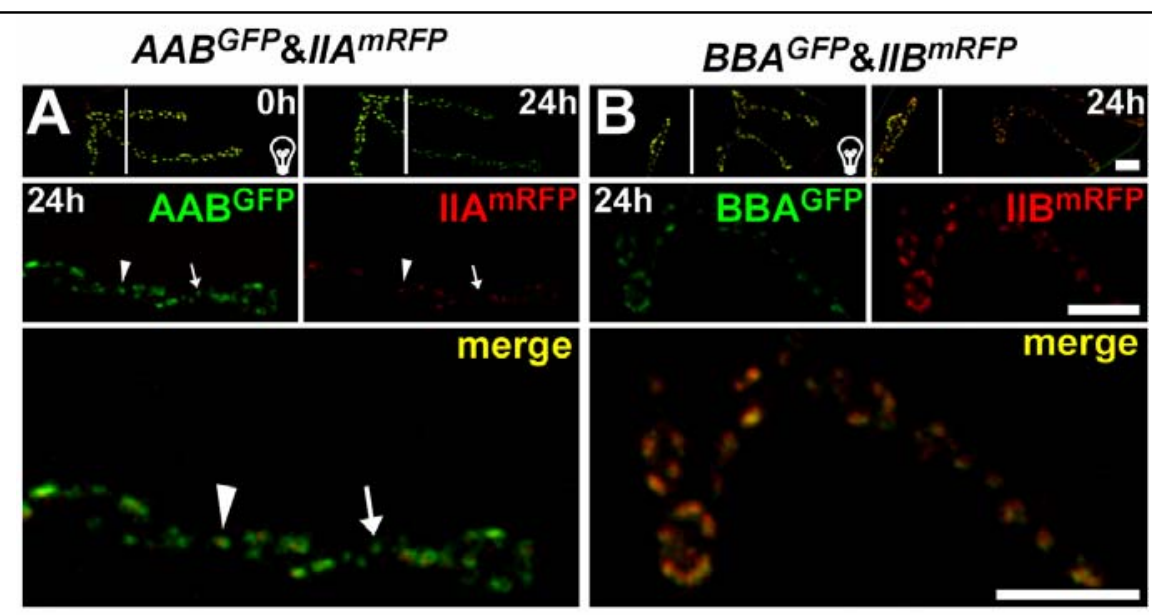

Fig. 42. The differential incorporation characteristics involve the receptor C-terminus - part II

FRAP experiments on early $3^{\text {rd }}$ instar larvae (NMJ27). Shown are the initial (Oh), the bleached (indicated by the bulb) and the recovered time point (24h). All transgenes were expressed in the

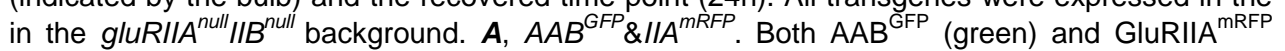
(red) showed a patchy recovery pattern (arrows and arrowheads) with $A A B^{\text {GFP }}$ FRAP being stronger and more consistent. $\boldsymbol{B}, B B A^{\text {GFP }}$ \&IIB $^{\text {mRFP }}$. Both BBA ${ }^{\text {GFP }}$ (green) and GluRIIB ${ }^{\text {mRFP }}$ (red) exhibited uniform FRAP over all PSDs with GluRIIB ${ }^{\text {mRFP }}$ showing a trend to stronger recovery. All images derived from confocal microscopy. Scale bars: $5 \mu \mathrm{m}$.

Taken together, principal PSD incorporation behavior is not dependent on the simultaneous presence of both the GluRIIA and IIB complex, while the CTD of GluRIIA promotes suppression of GluRIIA incorporation at mature type PSDs.

\subsubsection{GluRIIA is needed to allow efficient growth of PSDs and to define mature PSD size}

Despite the absence of growth related glutamate receptor incorporation accomplished by GluRIIA (Rasse et al., 2005), PSD populations at "GluRIIB-only NMJs" (Fig. 35C and Fig. 41B) can still form. However, the number of synapses forming per GluRIIB-only NMJ is reduced when compared to a GluRIIA-only NMJ (Fig. 34E-H), and further structural plasticity upon NMJ experience is inhibited in this situation (Sigrist et al., 2003; Zhong and Wu, 2004).

Differences in PSD growth could principally underlie this restricted structural plasticity of GluRIIB-only NMJs. Thus, the effect of the receptor composition on PSD growth was analyzed by in vivo imaging of NMJs over 12h, comparing GluRIIB and

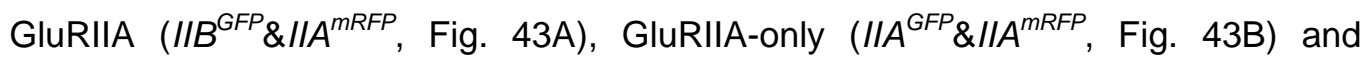
GluRIIB-only (IIB ${ }^{\text {GFP }} \& / I B^{m R F P}$, Fig. 43C) NMJs. At $\left\|B^{G F P} \&\right\| A^{m R F P}$ and $I I A^{G F P} \& \| A^{m R F P}$ NMJs, the PSD size distribution was similar with a slight and significant shift towards higher PSD size at GluRIIA-only NMJs ( $p=0.014$ ). GluRIIB-only NMJs (Fig. 43C), however, exhibited an untypical PSD size distribution, with two maxima, one at lower and one at larger PSD size (mean PSD size: $I I B^{G F P} \& / I A^{m R F P}, 0.323 \pm 0.008 \mu m^{2}$, 
$\mathrm{n}=505,8 N M J s, \quad I / A^{G F P} \& \| A^{m R F P}, \quad 0.346 \pm 0.007 \mu m^{2}, \mathrm{n}=559,8 N M J s, \quad I / B^{G F P} \& / I B^{m R F P}$, $\left.0.325 \pm 0.010 \mu \mathrm{m}^{2}, \mathrm{n}=433,9 \mathrm{NMJ}\right)$. The mean PSD size was as well significantly reduced compared to $I I A^{G F P} \& I I A^{\text {mRFP }}(\mathrm{p}=0.010)$. These data are interesting in the light of the observation that $I I B^{G F P} \& I I A^{\text {mRFP }}$ larvae showed a population of particularly small GluRIIB-rich synapses (Fig. 37D, table).

Finally, PSD growth dynamics were analyzed. The relation of PSD growth to the initial PSD size appeared similar between $I / B^{G F P} \& / I A^{m R F P}$ (Fig. 43A) and $I I A^{G F P} \& I I A^{m R F P}$ (Fig. 43B) NMJs, with PSD growth dropping steeply with increasing PSD size. In contrast, a less pronounced drop of PSD growth could be observed at GluRIIB-only NMJs (Fig. 43C). In result, linear regression analysis indicated a larger maximum PSD size (after extrapolation) in this situation. Thus, while initial PSD growth is delayed in the absence of GluRIIA, with many small PSDs accumulating, the stop of PSD growth at a definite size seems defective in this situation as well. In other words, GluRIIA apparently fulfills two functions, promoting growth of nascent PSDs, and mediating a stop of further PSD growth once a sufficient PSD size has been reached. In the absence of GluRIIA, the number of PSDs per NMJ is reduced (Fig. 34H) and cannot be any longer adapted on different needs (Sigrist et al., 2003).
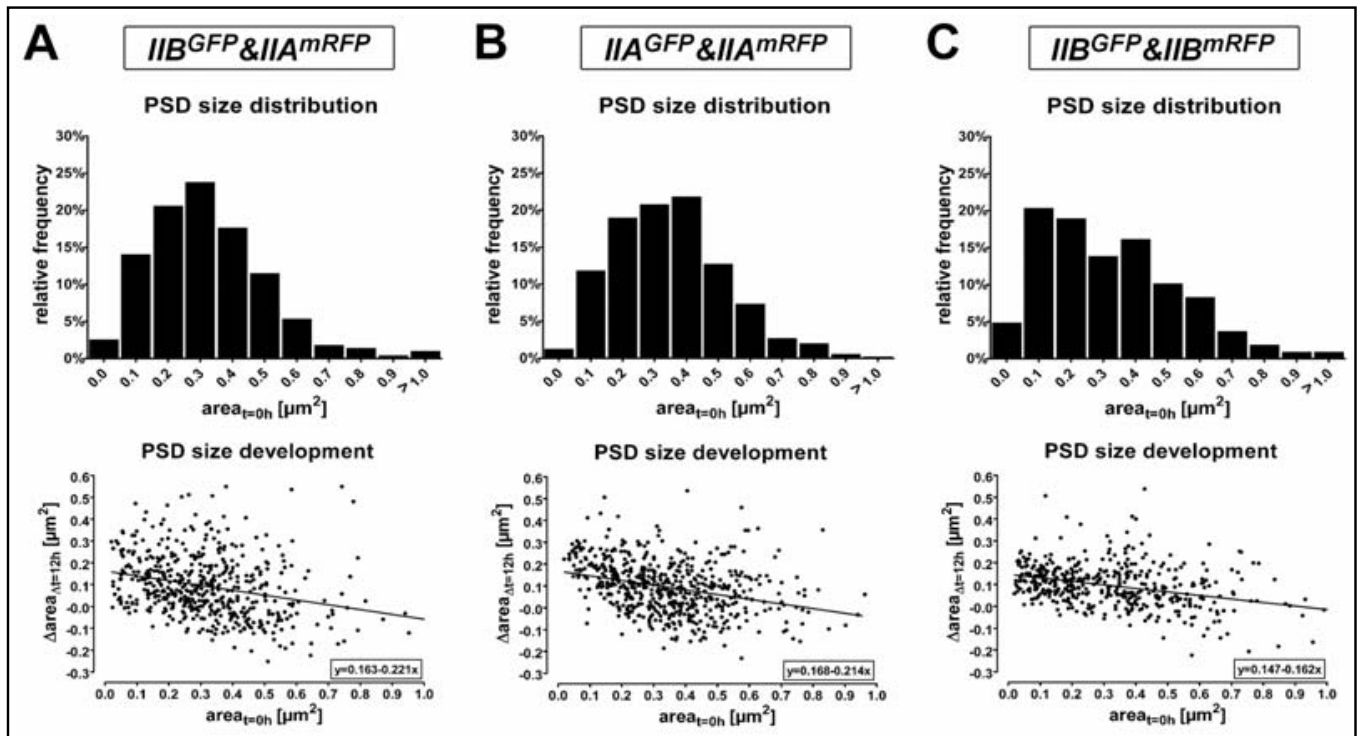

\section{Fig. 43. GluRIIA limits PSD growth}

Quantitative data resulting from confocal in vivo imaging of early $3^{\text {rd }}$ instar larvae $\left(\mathrm{NMJ} 27,25^{\circ} \mathrm{C}\right)$ expressing fluorescence-labeled glutamate receptor subunits in the embryonic lethal gluRIIA $A^{\text {null }} \| B^{\text {null }}$ background. Single identified PSDs at $\left\|B^{G F P} \&\right\| A^{\text {mRFP }}(\boldsymbol{A}, \mathrm{n}=505,8 \mathrm{NMJs})$, $\left\|A^{G F P} \&\right\| A^{\text {mRFP }}(B, \mathrm{n}=559,8 N M J s)$ and $I I B^{G F P} \& / I B^{m R F P}(C, \mathrm{n}=433,9 N M J s)$ NMJs were recorded and re-imaged $12 \mathrm{~h}$ after the first time point. The initial PSD size ( $\left(\mathrm{area}_{\mathrm{t}=0 \mathrm{~h}}\right)$ as well as the change in PSD size during the $12 \mathrm{~h}$ of development $\left(\Delta\right.$ area $\left._{\Delta \mathrm{t}=12 \mathrm{~h}}\right)$ were quantified. In comparison to $I I B^{G F P} \& I I A^{m R F P}(\boldsymbol{A})$, the size distribution of $I I A^{G F P} \& \| A^{m R F P}(\boldsymbol{B})$ was slightly up-shifted, while for $\left\|B^{G F P} \&\right\| B^{m R F P}(C)$ small PSDs were most frequent. The change in PSD size in relation to the initial size was most diverse for $\left\|B^{G F P} \&\right\| A^{\text {mRFP }}(\boldsymbol{A})$ and least diverse for $I B^{G F P} \& I^{\text {mRFP }}(\boldsymbol{C})$. While in the presence of GluRIIA the absolute growth of immature PSDs tended to be increased $(\boldsymbol{A}, \boldsymbol{B}$, axis intercept), PSD growth seemed uncontrolled in the absence of GluRIIA (C, slope). 


\subsubsection{GluRIIA dominates synaptic transmission}

Above it was shown that during larval NMJ development, PSDs grew to a defined size and, simultaneously, PSD receptor composition converged towards a balanced ratio of GluRIIA and GluRIIB. The question remains whether this behavior was solely explained by a "biochemical" assembly principle, or whether also the physiological properties of these receptor assemblies might be relevant here. Previously, both NMJ glutamate receptor complexes were shown to differ strongly in transmission (DiAntonio et al., 1999; Chen and Featherstone, 2005). In single channel recordings of extrasynaptic receptors, GluRIIB complexes desensitized about 10-fold faster than GluRIIA complexes (DiAntonio et al., 1999). Moreover, genetic elimination of GluRIIA (Petersen et al., 1997; Reiff et al., 2002) or over-expression of GluRIIB (DiAntonio et al., 1999) drastically reduces spontaneous responses, being a measure for the glutamate-gated ionic conductance at individual PSDs.

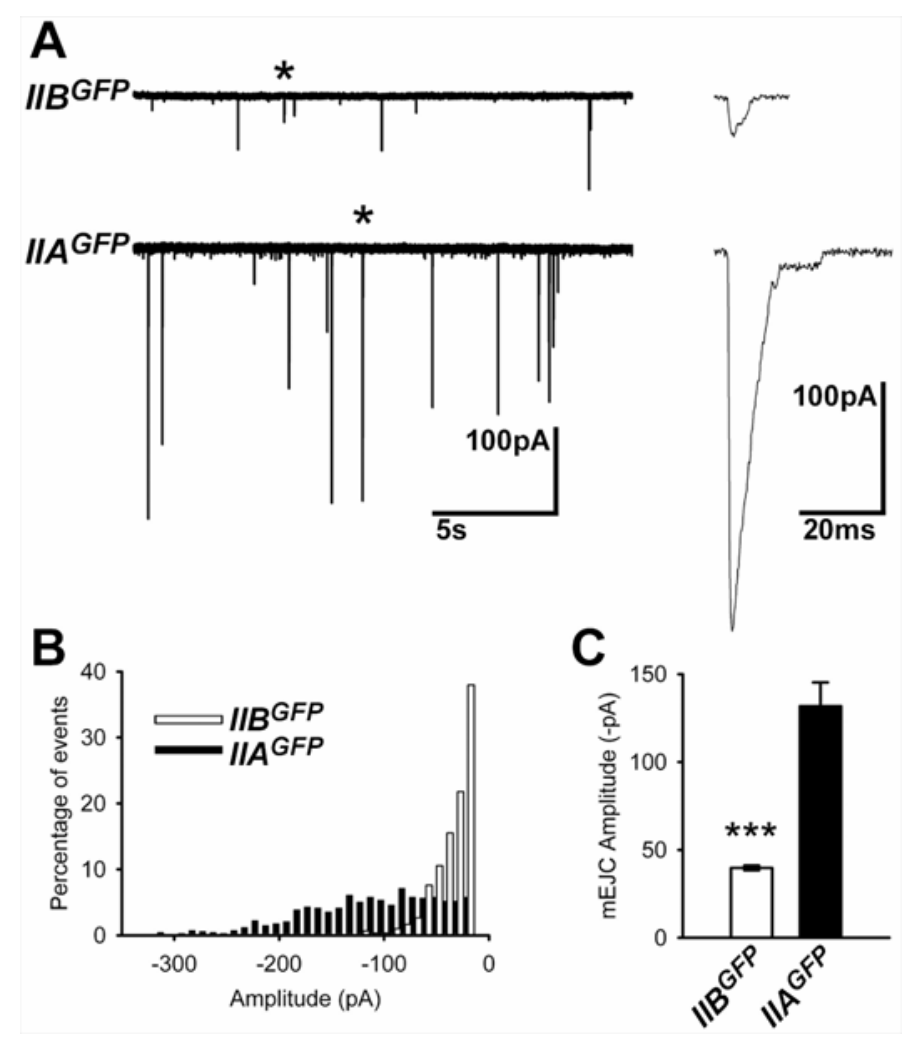

Fig. 44. Physiological characterization of GluRIIA and GluRIIB complexes

$\boldsymbol{A}$, Shown are traces of patch clamp recordings on embryos (20-22h old, NMJ 6/7) expressing either one copy of gluRIIB ${ }^{G F P}\left(I I B^{G F P}\right)$ or gluRIIA $A^{G F P}\left(I I A^{G F P}\right)$ to rescue the lethal gluRIIA $A^{\text {null } I I B^{\text {null }}}$ background. The asterisks indicate the miniature evoked junctional currents (mEJCs), which are shown in higher magnification (right). $\boldsymbol{B}$, Histograms of the $\mathrm{mEJC}$ amplitudes uncover a wide and accordingly a narrow distribution for $I I A^{G F P}$ (max. 316pA) and IIB ${ }^{G F P}$ (max. 124pA), respectively. $C$, The mean mEJC of $I I B^{G F P}$ is significantly decreased compared to IIA ${ }^{G F P}$ (IIB ${ }^{G F P}, 39.7 \pm 1.4 \mathrm{pA}$, $\left.\mathrm{n}=7, I I A^{G F P}, 131.7 \pm 13.5 \mathrm{pA}, \mathrm{n}=6,{ }^{\star \star *} \mathrm{p}<0.001\right)$. 
In order to measure spontaneous synaptic PSD currents with ideal signal-noise ratio from either GluRIIA-only or GluRIIB-only NMJs, embryonic muscles were subjected to patch clamp recording (see 3.4.1). In fact, spontaneous currents at GluRIIA-only PSDs were more than threefold higher than at GluRIIB-only PSDs (Fig. 44A,B,C; IIB $\left.^{\text {GFP }}, 39.7 \pm 1.4 p A, n=7, I I A^{G F P}, 131.7 \pm 13.5 p A, n=6, p<0.001\right)$. Thus, the remarkable differences in eEJCs between GluRIIA-only and GluRIIB-only NMJs (see Fig. 34C,D) are to a large extent due to an enormous difference in the conductance of synaptic GluRIIA and GluRIIB channels. Balancing the receptor composition of the PSD might therefore be important to normalize conductance during PSD maturation. The specific PSD conductance might in turn contribute to regulate the differential incorporation of the two glutamate receptor complexes. Insufficient conductance might stimulate incorporation of the high conductance species (GluRIIA) and vice versa high conductance due to mature GluRIIA content might stop further GluRIIA and favor GluRIIB incorporation.

\subsubsection{GluRIIA PSD content is selectively enhanced after blockade of presynaptic glutamate release}

If postsynaptic conductance was involved in the differential receptor incorporation, an interference with presynaptic glutamate release could affect the receptor composition of PSDs. Thus, mosaic presynaptic expression of tetanus toxin light chain (TNT) using ok319-ga/4 to suppress evoked glutamate release (Sweeney et al., 1995) was combined with in vivo imaging of GluRIIB ${ }^{\text {GFP }}$ and GluRIIA ${ }^{\text {mRFP }}$ (in gluRIIA $A^{\text {null }} \| B^{\text {null }}$ background) (Fig. 46A $\left\|B^{G F P} \&\right\| A^{\text {mRFP }}$, NMJ 14; Fig. 46B: $\left.I I B^{G F P} \& I I A^{m R F P}+o k 319-t n t\right)$. As ok319-gal4 did not express in the motoneuron innervating muscle 27 (usually used in this study for in vivo imaging), NMJs of the ventral-longitudinal muscle 14 were analyzed for this experiment (Fig. 45).

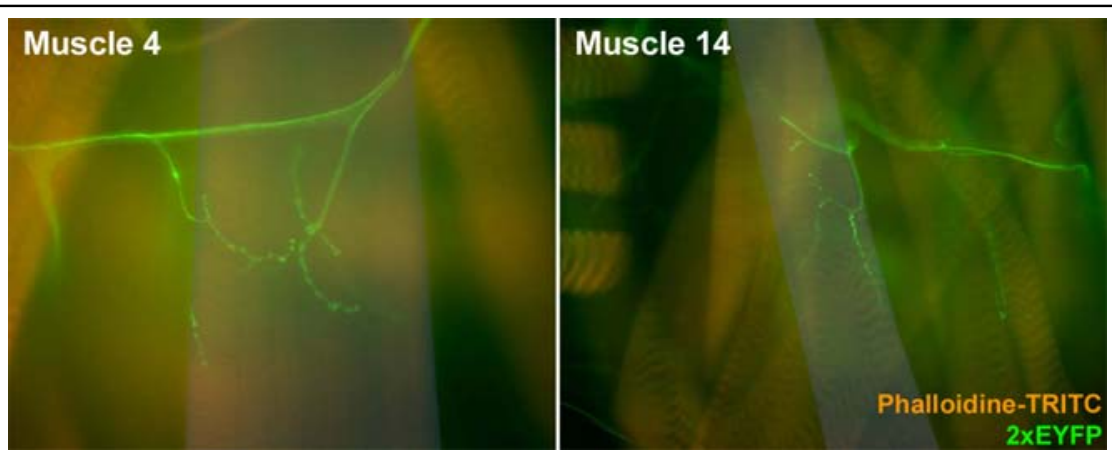

Fig. 45. Expression pattern of ok319-gal4

Phalloidine (TRITC-coupled, orange) staining of $3^{\text {rd }}$ instar larvae expressing 2XEYFP (green) ectopically with the ok319-gal4 driver. ok319-gal4 showed presynaptic expression at the NMJ of the ventral-longitudinal muscle 4 (as well at the NMJs $6 / 7$ and $12 / 13$, not shown) and of the ventral-oblique muscle 14 but not of the ventral-acute muscle 27 (not shown). 
PSDs were classified according to their size and receptor composition (ratio r). At TNT expressing NMJs (Fig. 46B,D,F), small PSDs $\left(<0.2 \mu \mathrm{m}^{2}\right)$ were particularly rich of GluRIIA $^{\text {mRFP }}(r<0.8)$, while the receptor composition at medium and large PSDs (0.2$0.4 \mu \mathrm{m}^{2}$ and $>0.4 \mu \mathrm{m}^{2}$ ) appeared essentially unaffected compared to controls (Fig. $46 A, C, E)$. To see whether a difference in GluRIIA incorporation was responsible, again FRAP experiments were performed.

\section{Fig. 46. PSD composition after blockage of presynaptic release}

$\boldsymbol{A}$ and $\boldsymbol{B}$, In vivo imaging of GluRIIB $^{\text {GFP }}$ (green) and GluRIIA ${ }^{\text {mRFP }}$ (red) with (B) or without $(\boldsymbol{A})$ a concomitant activity blockage with ok319-tnt (gluRIIA $A^{\text {null } I I B^{\text {null }}}$ background, NMJ $14,25^{\circ} \mathrm{C}$ ). $\boldsymbol{C}$ and $\boldsymbol{D}$, Shown are the initial size and ratio $r\left(\right.$ Int $_{\text {rel }}\left(I I B^{G F P}\right) / I^{\text {nnt }}$ rel $\left(I I A^{\mathrm{mRFP}}\right)$ ) of individual PSDs (from $\boldsymbol{A}$ and $\boldsymbol{B}$ ).

$\boldsymbol{E}$ and $\boldsymbol{F}$, After activity suppression $(\boldsymbol{E})$, small PSDs $\quad\left(<0.2 \mu \mathrm{m}^{2}\right) \quad$ were predominantly rich of GluRIIA $^{\text {mRFP }} \quad(r<0.8)$. In comparison to the control $(\boldsymbol{F})$, the other PSD size classes $\left(0.2-0.4 \mu \mathrm{m}^{2}\right.$ and $>0.4 \mu \mathrm{m}^{2}$ ) showed a nondistinguishable distribution of the three ratio classes.

$\boldsymbol{G}$ and $\boldsymbol{H}$, FRAP experiments with $24 \mathrm{~h}$ recovery after bleaching of both the GFP and mRFP channel. IIB ${ }^{G F P} \& \| A^{\text {mRFP }}$

(G): as observed on NMJ 27, PSDs showed consistent incorporation of GluRIIB GFP (green) and either faint (arrows) or intense (arrowheads) recovery of GluRIIA $^{\text {mRFP }}$ (red). IIB ${ }^{G F P} \& / I A^{\text {mRFP }}+$ ok319-tnt $(\boldsymbol{H})$ : compared to the situation without activity blockage (G), GluRIIB ${ }^{\text {GFP }}$ recovery seemed weaker. GluRIIA $^{\text {mRFP }}$ maintained its non-uniform FRAP pattern (arrows and arrowheads) with an increase of the overall recovery. The presented images derived from confocal microscopy of $3^{\text {rd }}$ instar larvae. Scale bars: $5 \mu \mathrm{m}$.
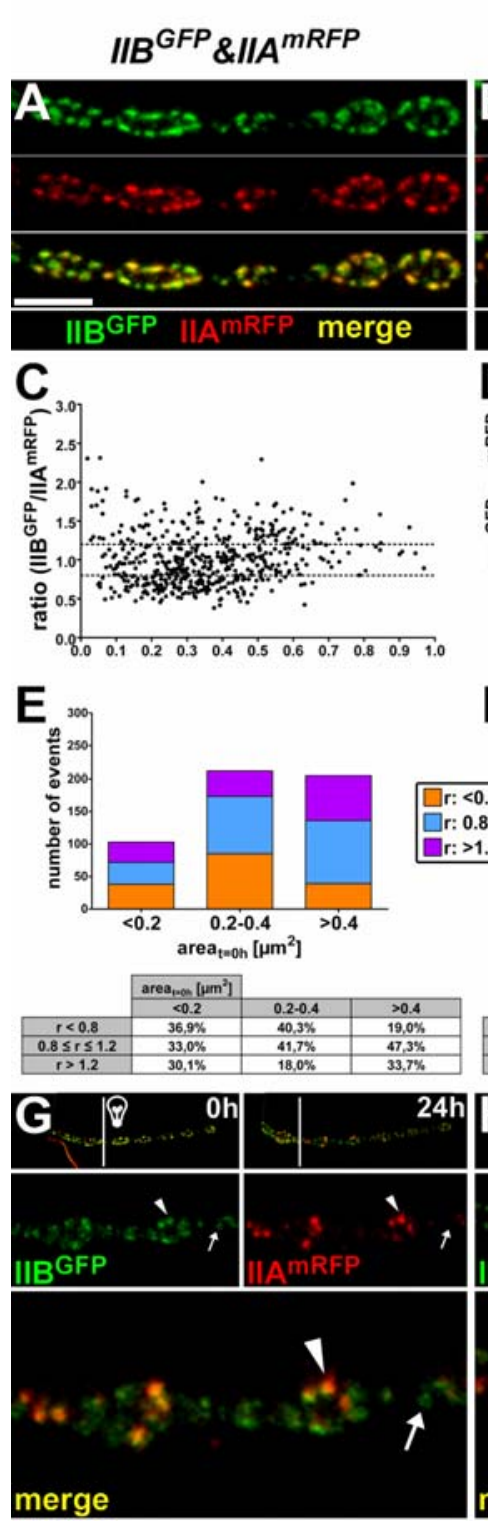

activity suppression with TNT
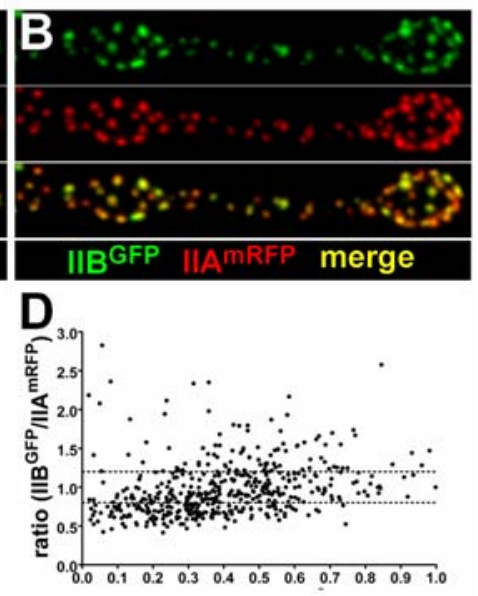

$\mathbf{F}$
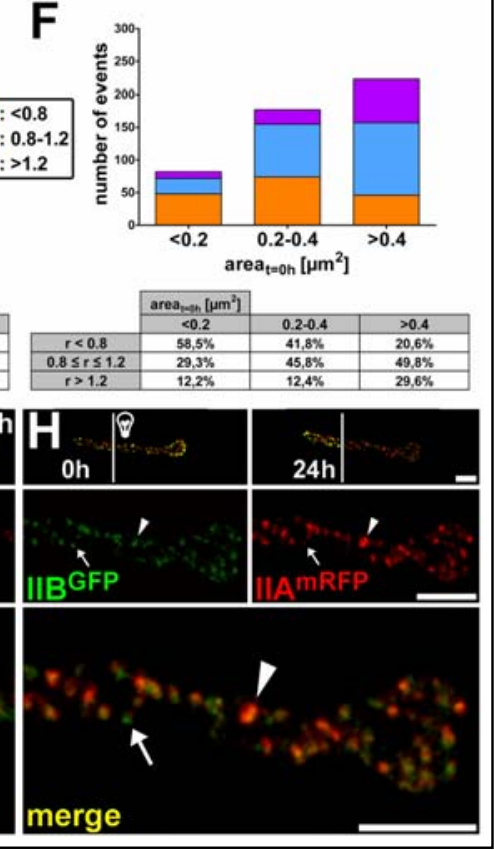

At the control NMJs (Fig. 46G), recovery of GluRIIA ${ }^{\text {mRFP }}$ was very faint at a large fraction of PSDs but very intense at a few PSDs while GluRIIB ${ }^{\text {GFP }}$ exhibited uniform 
FRAP, as typically observed throughout this study (Fig. 38, Fig. 39A,C, Fig. 40A). However, more PSDs appeared to efficiently incorporate GluRIIA ${ }^{\text {mRFP }}$ at TNT expressing NMJs (Fig. 46H), also obvious after quantitative analysis (Fig. 39E), which uncovered a rather Gaussian-like recovery distribution (fit value: 0.963). For GluRIIB $^{\text {GFP }}$ an atypically low Gaussian fit (fit value: 0.892) could be observed. This can be explained by the reduced FRAP, which was often below the detection threshold (corresponding to a recovery rate of about $40 \%$ ).

Further FRAP experiments addressed whether the influence of TNT expression was

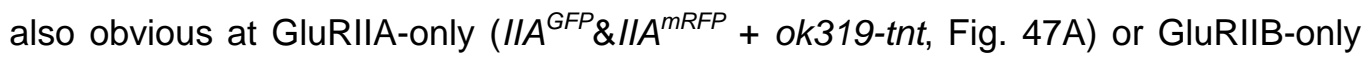
NMJs (IIB ${ }^{\text {GFP }}$ \&IIB ${ }^{\text {mRFP }}+$ ok319-tnt, Fig. 47B). Again, FRAP of GluRIIA ${ }^{\text {mRFP }}$ appeared slightly stronger than in non-TNT expressing controls (Fig. 41A), whereas recovery of GluRIIB $^{\text {mRFP }}$ appeared unchanged or even weaker in comparison to controls (Fig. 41B). As before, also in the presence of TNT GluRIIA and GluRIIB incorporation behavior appeared unaffected by the absence of the respective other receptor complex type.

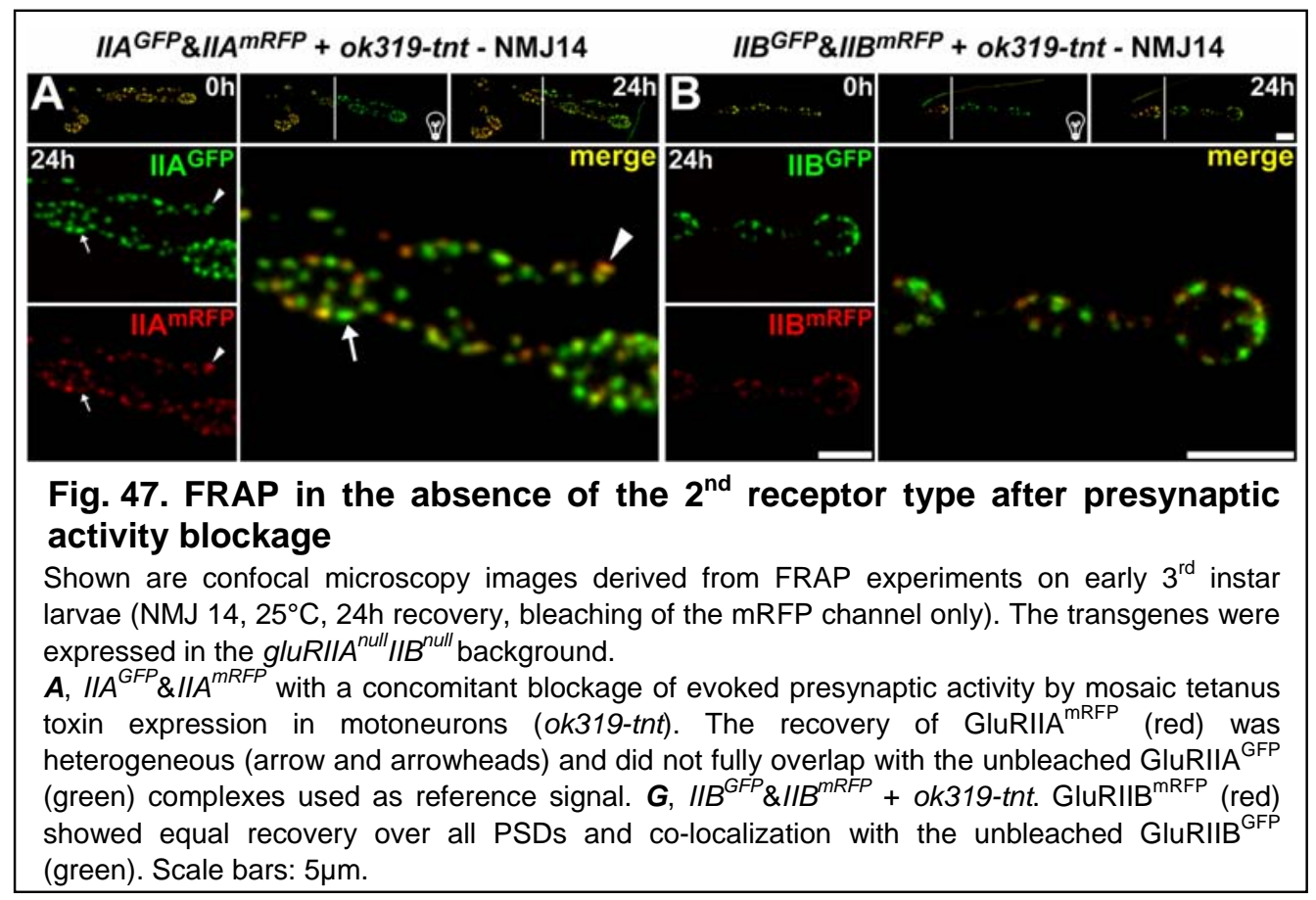

So far, the data indicate that suppression of glutamate release and thus ionic conductance provokes a further incorporation of GluRIIA, leading to increased GluRIIA content particularly at small immature PSDs. Increased incorporation of GluRIIA in turn should provoke an increase in PSD growth (Rasse et al., 2005). In fact, after TNT expression (Fig. 46H), the mean PSD size (resulting from a maximum overlay of both GluRIIA ${ }^{\text {mRFP }}$ and GluRIIB ${ }^{\text {GFP }}$ ) was significantly increased (Fig. 48C, 
$\left\|B^{G F P} \&\right\| A^{m R F P}, N M J 14,0.362 \pm 0.008 \mu m^{2}, n=519,9 N M J s, I I B^{G F P} \& \| A^{m R F P}+o k 319-t n t$, $0.402 \pm 0.010 \mu m^{2}, n=482,8$ NMJs, $p=0.0034$ ). Finally, GluRIIA and GluRIIB were also visualized by antibody stainings (Fig. 48A,B) using a different NMJ (NMJ 4, which also shows ok319-gal4 expression, see Fig. 45). In fact, the GluRIIA level per PSD was increased while GluRIIB remained unchanged after TNT expression (Fig. 48C;

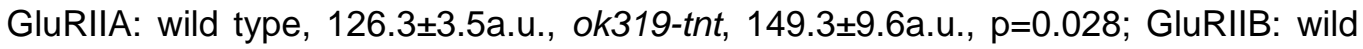

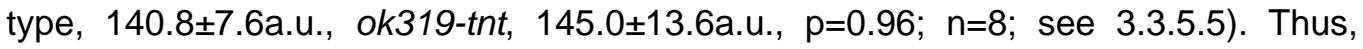
suppression of presynaptic release provoked a specific increase in GluRIIA content and PSD size.

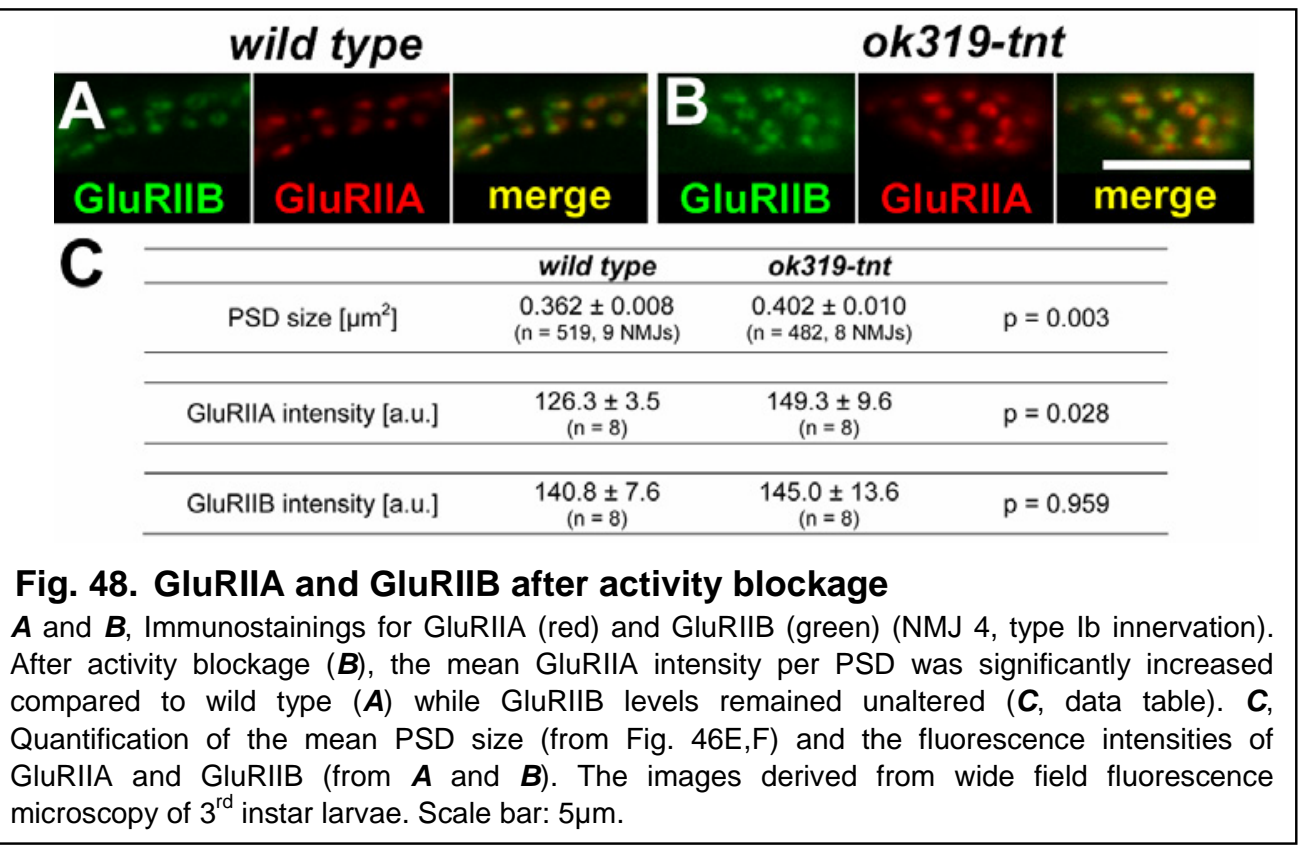

\subsubsection{Receptor content per PSD can be down-regulated to allow the formation of additional synapses during experience-dependent plasticity}

Finally, the question was addressed how glutamate receptor content per PSD would be organized when the $\mathrm{NMJ}$ as a system was challenged towards producing additional synapses. Previously, it was shown that an elevation of the environmental temperature is associated with increased locomotion (Sigrist et al., 2003). Thereby, the number of synaptic boutons and the $\mathrm{NMJ}$ transmission strength was shown to be considerably increased (Sigrist et al., 2003; Zhong and Wu, 2004). Interestingly, antibody stainings of wild type NMJs (muscle 4) exhibited an accompanying drastic reduction of both GluRIIA and GluRIIB levels at PSDs when the temperature was shifted from $25^{\circ} \mathrm{C}$ (Fig. 49A) to $29^{\circ} \mathrm{C}$ (Fig. 49B) (Fig. 49C; GluRIIA: $25^{\circ} \mathrm{C}$, 173.8 \pm 13.8a.u., $n=12,29^{\circ} \mathrm{C}, 109.8 \pm 12.4$ a.u., $n=11, p=0.005$; GluRIIB: $25^{\circ} \mathrm{C}$, 
183.9 \pm 12.0 a.u., $n=12, \quad 29^{\circ} \mathrm{C}, 102.3 \pm 16.8$ a.u., $\left.n=11, \quad \mathrm{p}=0.005\right)$. Despite the coordinated reduction of both GluRIIA and GluRIIB, the amplitude of miniature excitatory currents is not significantly altered (Sigrist et al., 2003). This regulation might either be mediated by changes in postsynaptic conductivity, e.g. by posttranslational modifications of GluRIIA complexes or changes in the per vesicle glutamate content (Steinert et al., 2006) (see discussion).

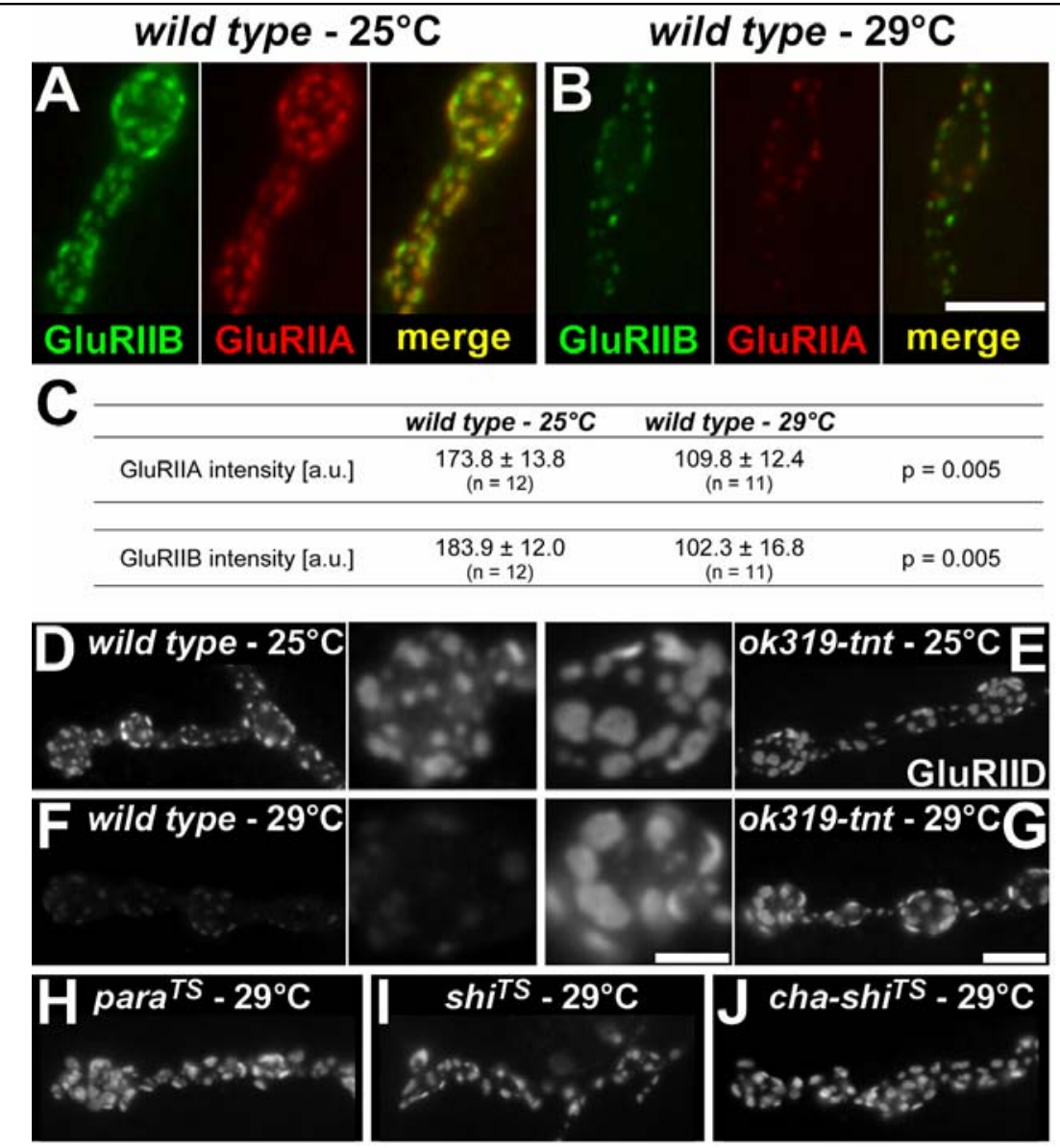

Fig. 49. PSDs during experience-dependent plasticity

$A-C$, Immunostainings for GluRIIA (red) and GluRIIB (green) of wild type $3^{\text {rd }}$ instar NMJs (muscle 4) permanently raised either at $25^{\circ} \mathrm{C}(\boldsymbol{A})$ or $29^{\circ} \mathrm{C}(\boldsymbol{B})$. Both GluRIIA and GluRIIB were drastically reduced at $29^{\circ} \mathrm{C}$ (C, data table).

$D-J$, PSDs (NMJ 6/7) stained for GluRIID. In comparison to wild type (D), PSDs of animals with mosaic expression of tetanus toxin light chain in motoneurons using ok319-gal4 (E, ok319-tnt) showed an increased size (both raised at $25^{\circ} \mathrm{C}$ ). Raising wild type offspring at $29^{\circ} \mathrm{C}(\boldsymbol{F})$ resulted in a reduction of GluRIID immunoreactivity. In contrast, ok319-tnt $(\boldsymbol{G})$, para ${ }^{T S 1}(\boldsymbol{H})$ and shi $^{T^{T S 1}}(\boldsymbol{I})$ PSDs $\left(\right.$ at $29^{\circ} \mathrm{C}$ ) appeared increased in size and showed no reduction of GluRIID. The same could be observed when shi ${ }^{T S 1}$ was expressed in cholinergic motoneurons using cha-ga/4 ( $J$, $29^{\circ} \mathrm{C}$ ). All images derived from wide field fluorescence microscopy under equal illumination conditions, respectively. Scale bars: $5 \mu \mathrm{m}, \mathrm{G}$, enlarged panel, $2 \mu \mathrm{m}$.

The down-regulation of glutamate receptor levels at PSDs following experiencedependent plasticity was equally present in immunostainings for the subunit GluRIID, likely to be part of both GluRIIA and GluRIIB complexes (Fig. 34B). PSDs (NMJ 6/7) 
of animals consistently raised at $29^{\circ} \mathrm{C}$ (Fig. 49F) showed strongly reduced levels of GluRIID compared to the $25^{\circ} \mathrm{C}$ control (Fig. 49D). To address whether this reduction was mediated by the temperature increase and the associated potentiation of presynaptic release (Sigrist et al., 2003), several situations of affecting (predominantly) evoked vesicle release at presynaptic NMJ terminals were examined. Expression of tetanus toxin light chain with the mosaic motoneuron driver ok319-gal4 (ok319-tnt) (Sweeney et al., 1995) at $29^{\circ} \mathrm{C}$ (Fig. 49G) did not result in diminished glutamate receptor levels compared to $25^{\circ} \mathrm{C}$ (Fig. 49E). Compared to wild type, PSDs at ok319-tnt NMJs appeared clearly increased in size. Similarly, paralytic mutants para $^{T S 1}$ (para encodes a functionally predominant class of sodium channels in Drosophila neurons) (Loughney et al., 1989) raised at $29^{\circ} \mathrm{C}$ (Fig. $49 \mathrm{H}$ ) did not exhibit a reduction in glutamate receptor density at PSDs. An identical finding could be obtained when temperature-sensitive, dominant-negative Dynamin (UAS-shit ${ }^{T S 1}$ ) was expressed at $29^{\circ} \mathrm{C}$ in cholinergic neurons upstream of motoneurons with the cha-gal4 driver (cha-shi ${ }^{\text {TS1 }}$, Fig. 49J) (Salvaterra and Kitamoto, 2001). In addition, the PSD size tended be increased compared to wild type (Fig. 49F) when both spontaneous and evoked transmission were impaired raising shi $i^{T S 1}$ animals at $29^{\circ} \mathrm{C}$ (Fig. 49I) (Koenig et al., 1983).
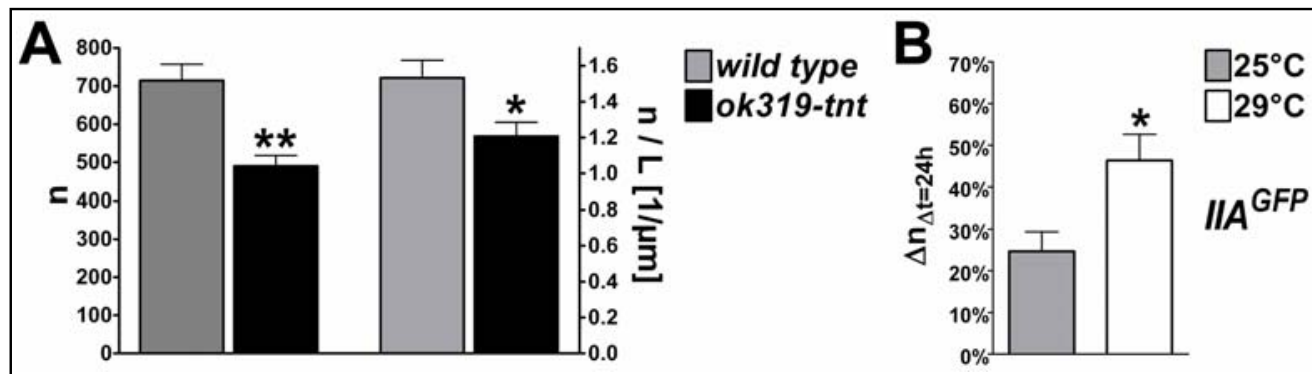

Fig. 50. Activity and long-term NMJ plasticity

$\boldsymbol{A}$, Synapses at NMJ $6 / 7$ of $3^{\text {rd }}$ instar larvae (raised at $25^{\circ} \mathrm{C}$ ) were manually counted from Nc82 immunolabelings. The synapse number (n) measured for ok319-tnt was clearly reduced compared to wild type (wild type, 715 \pm 42 , $n=9$, ok319-tnt, 490 $\pm 27, n=7, p=0.002$ ). This reduction also persisted after normalization to the respective segment length ( $\mathrm{L}$; see 7.5$)(\mathrm{n} / \mathrm{L}$; wild type, $1.53 \pm 0.10$ per $\mu \mathrm{m}, \mathrm{n}=9$, ok319-tnt, $1.21 \pm 0.08$ per $\mu \mathrm{m}, \mathrm{n}=7, \mathrm{p}=0.031)$. $\boldsymbol{B}$, Relative increase in the PSD number $\left(\Delta \mathrm{n}_{\Delta \mathrm{t}=24 \mathrm{~h}}\right)$ determined via in vivo imaging of $I I A^{G F P} 3^{\text {rd }}$ instar larvae (NMJ 27, $\Delta \mathrm{t}=24 \mathrm{~h}$ ) that were either kept at $25^{\circ} \mathrm{C}$ or shifted to $29^{\circ} \mathrm{C}$ after the first imaging session. The temperature shift to $29^{\circ} \mathrm{C}$ resulted in increased formation of novel PSDs $\left(25^{\circ} \mathrm{C}, 24.7 \pm 4.7 \%, \mathrm{n}=9\right.$, $\left.29^{\circ} \mathrm{C}, 46.3 \pm 6.3 \%, \mathrm{n}=11, \mathrm{p}=0.019\right)$.

Finally, the overall synapse number of wild type and ok319-tnt NMJs was compared (NMJ 6/7, 25 ${ }^{\circ} \mathrm{C}$ ). After activity blockage with TNT the synapse number was significantly decreased in comparison to the wild type control (Fig. 50A; for data see legend). In contrast, it was reported previously (Sigrist et al., 2003; Zhong and Wu, 2004) that NMJs of wild type larvae reared at $29^{\circ} \mathrm{C}$ harbored significantly more synapses than the $25^{\circ} \mathrm{C}$ controls. To study acute effects of elevated temperature and 
with it activity on synaptic growth, IIA GFP larvae (gluRIIA $A^{\text {GFP }}$ expressed in the gluRIIA ${ }^{\text {null }} / I B^{\text {null }}$ background) were subjected to in vivo imaging (NMJ 27). After the first imaging session the larvae were either kept at $25^{\circ} \mathrm{C}$ or shifted to $29^{\circ} \mathrm{C}$ until the second time point (24h later). For both time points the PSD number was determined and the relative increase in the PSD number $\left(\Delta n_{\Delta t=24 h}\right)$ was calculated. Notably, NMJs of animals shifted to $29^{\circ} \mathrm{C}$ produced nearly twice as many novel PSDs (Fig. 50B; $\left.\Delta \mathrm{n}_{\Delta \mathrm{t}=24 \mathrm{~h}}, 25^{\circ} \mathrm{C}, 24.7 \pm 4.7 \%, \mathrm{n}=9,29^{\circ} \mathrm{C}, 46.3 \pm 6.3 \%, \mathrm{n}=11, \mathrm{p}=0.019\right)$.

In summary, an increase in the raising temperature (from $25^{\circ} \mathrm{C}$ to $29^{\circ} \mathrm{C}$ ) provoked a drastic down-regulation of glutamate receptor levels that was obviously mediated by potentiated presynaptic activity. Going along with the decreased glutamate receptor density per PSD, more novel PSDs could be established. Contrary to it, activity suppression did not reduce but rather elevated the glutamate receptor number per PSD. Moreover, the number of PSDs per NMJ was clearly decreased. Thus, glutamate receptor levels at PSDs, the final PSD size and the overall number of synapses forming seem to be regulated by presynaptic activity. 


\section{Discussion}

\subsection{Non-NMDA type glutamate receptors are essential for maturation but not for initial assembly of synapses at Drosophila NMJs}

A detailed molecular and cell-biological insight into the formation of glutamatergic synapses is important for understanding the development of excitatory neuronal circuits and also the process of long-term information storage in the CNS (Chklovskii et al., 2004). So far, studies on cultivated brain neurons analyzed mechanisms of glutamate receptor trafficking during synapse formation and have suggested a temporal sequence of pre- and postsynaptic assembly (Washbourne et al., 2002; Gundelfinger et al., 2003; Bresler et al., 2004). However, whether in turn the process of incorporating glutamate receptors is needed for the establishment of synaptic structures was hardly addressed.

\subsubsection{A transmission independent role of glutamate receptors in postsynaptic maturation}

The relationship between neurotransmitter receptor incorporation and synapse assembly was addressed by genetically reducing or eliminating the expression of all neurotransmitter receptors at a certain synapse type. Consequences of eliminating all postsynaptic glutamate receptors expressed at a specific glutamatergic synapse had not been described previously. Here it was shown that a lack of glutamate receptors provoked a specific block in the molecular and ultrastructural maturation of PSDs.

Notably, loss of transmission due to the loss of glutamate receptor complexes seemed not involved, based on the fact that neither blocking synaptic transmission (Fig. 26C,D,I,J) nor affecting glutamate binding by site-directed mutagenesis (Fig. 26K) did provoke similar defects. Thus, consistent with studies in other synaptic systems (Harris, 1980; Verhage et al., 2000; Baines et al., 2001; Misgeld et al., 2002; Varoqueaux et al., 2002; Heeroma et al., 2003) ionic transmission through the postsynaptic neurotransmitter receptors does not appear essential for principal synapse assembly. Instead, the data clearly imply that a critical level of glutamate receptor protein is needed to allow synapse maturation.

\subsubsection{Ultrastructural and molecular maturation of NMJ synapses requires glutamate receptors}

A model for the maturation of individual NMJ synapses in either presence or absence of postsynaptic glutamate receptors is given in Fig. 51. At glutamate receptor deprived synapses, synaptic vesicles appeared normally distributed, and 
their activity mediated release appeared increased, likely as part of a compensation for reduced postsynaptic sensitivity. Moreover, functional active zones with presynaptic dense bodies still formed. Thus, active zones still assemble when the mature organization of synaptic membranes ("tight planar apposition") is not established. Consistently, previous work had shown that the formation of presynaptic dense bodies persisted even after genetic elimination of postsynaptic muscle cells (Prokop et al., 1996). On the contrary, active zone formation is severely affected in bruchpilot mutants, while the pre- and postsynaptic membranes remain tightly apposed (Kittel et al., 2006; Wagh et al., 2006).
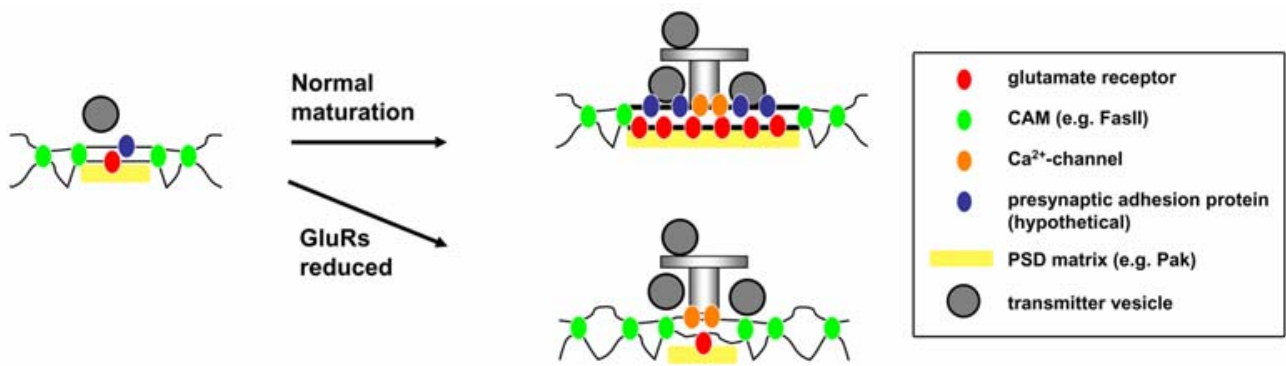

Fig. 51. Model: Maturation of PSDs requires glutamate receptors

The model shows the maturation of individual synapses with either normal or severely reduced/absent glutamate receptor levels. Initial definition of postsynaptic membranes (PAK accumulation) takes place even in the absence of glutamate receptors. However, further expansion of postsynaptic membrane domains including recruitment of electron density at both pre- and postsynaptic membranes and evenly-spaced adhesion between these membranes (apposition) fails when glutamate receptors are lacking. Thus, while presynaptic specializations mature, the PSD resides in an immature state and size with perisynaptic CAMs occupying principal PSD membranes.

At developing NMJs, newly forming "nascent" PSDs are characterized by small GluRIIA accumulations strictly co-localized with PAK kinase (Rasse et al., 2005). Even in the complete absence of glutamate receptors $\left(I / C^{\text {null }}\right.$ or $\| A^{\text {null }} / I B^{\text {null }}$, Fig. 25E,F), postsynaptic PAK patches, as typical for small nascent synapses still formed, indicating that principal cues for the definition of postsynaptic membrane patches persisted in this situation. Nonetheless, these PAK patches consistently failed to reach mature size (Fig. 23J,K). PAK, which mediates effects of Rho-GEF $\mathrm{dPIX}$ has been implicated in postsynaptic maturation, with PAK mutants showing a partial depletion of GluRIIA, and reduced SSR formation. However, neither pak nor dpix mutants have so far been reported to show defects in synaptic membrane apposition (Parnas et al., 2001; Albin and Davis, 2004). Thus, postsynaptic differentiation is not completely blocked in the absence of glutamate receptors. Instead, two postsynaptic "assembly modules" (PAK/dPIX signaling and glutamate receptor localization) appear only partly dependent on each other, with glutamate 
receptor localization being essential for PSD maturation but not for initial PSD assembly.

At the cholinergic mouse $\mathrm{NMJ}$, genetic deletion of the adult acetylcholine receptor subunit $\varepsilon(A C h R \varepsilon)$ led to severely reduced AChR density. Notably, a profound reorganization of $\mathrm{AChR}$-associated components of the postsynaptic membrane and cytoskeleton was observed in this situation (Missias et al., 1997).

\subsubsection{Glutamate receptor complexes and synaptic cell adhesion}

Synaptic membranes are electron-dense and apposed to each other leaving a cleft of consistent width, likely essential for robust timing and efficacy of neurotransmission. In contrast, perisynaptic membranes are less electron-dense and tend to undulate. At NMJs lacking glutamate receptors, Fasll/Dlg complexes ectopically remained at synaptic sites and membranes now appeared undulated, indicating perisynaptic type of membrane adhesion. Thus, glutamate receptors seem essential to establish the type of membrane adhesion found at the synapse, whereas usually perisynaptic adhesion molecules as Fasll mediate a qualitatively different type of membrane adhesion. Notably, undulation of perisynaptic membranes was impaired at NMJs lacking glutamate receptors leading to a less developed SSR (Fig. 24D,K; Fig. 27G). Moreover, boutons often appeared atypically round (Fig. 24C; Fig. 27D,E), further indicating that membrane-membrane adhesion is fundamentally affected at $\mathrm{NMJ}$ terminals lacking glutamate receptors.

Several classes of cell adhesion molecules (CAMs) have been implicated in mediating membrane adhesion at synapses, particularly trans-synaptic neurexinneuroligin pairs and cadherins (Murthy and De Camilli, 2003). The specific contributions of these synaptic CAMs during initial synapse assembly and maturation are under intense investigation. The data are consistent with the idea that the Cterminal, intracellular domains of glutamate receptors might engage in interactions with other PSD components, which in turn cluster postsynaptic CAM-type membrane proteins. These would then mediate interactions to cluster presynaptic CAMs or bind components of the extracellular matrix to allow synaptic membrane apposition (Fig. 52). Alternatively, direct interactions of glutamate receptors with other membrane protein complexes, as recently demonstrated for Stargazins/TARPs (Osten and Stern-Bach, 2006), might be involved.

So far, no CAM single mutant has been reported to provoke a defect in synaptic membrane apposition as severe as the one observed here for glutamate receptor mutant situations. Thus, multivalent interactions of the heterotetrameric glutamate 
receptor complexes as well as the redundant involvement of several CAM species might occur.

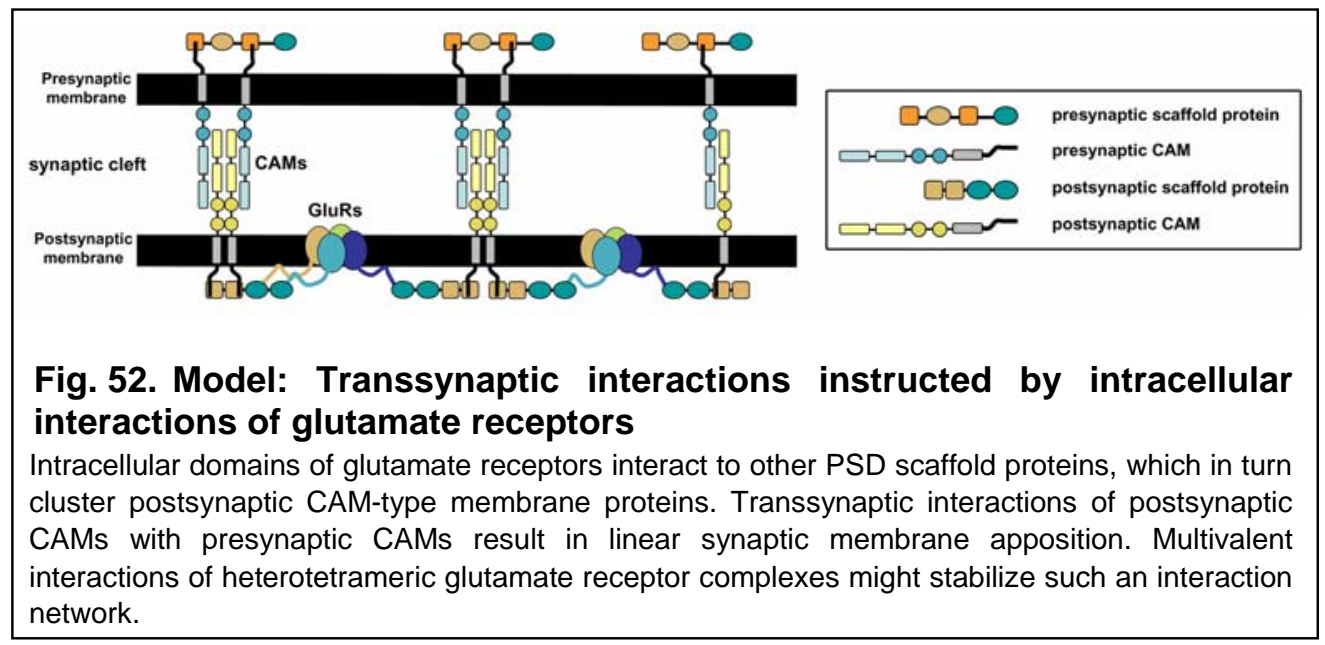

\subsubsection{Glutamate receptor levels in control of synapse formation}

PAK labeling suggested that initial steps in defining postsynaptic membranes persisted even in the total absence of glutamate receptors, while these initial assemblies could not mature on the ultrastructural level when glutamate receptors were lacking. Recently, in vivo imaging of photo-labeled GluRIIA at the developing $\mathrm{NMJ}$ uncovered that newly forming PSDs in fact grow by a continuous incorporation of glutamate receptors, whereby the accumulation of presynaptic active zone material (BRP) appeared slightly delayed. Thereby, the entry of GluRIIA - likely derived from cell wide plasma membrane pools via lateral diffusion - directly correlated with PSD growth. Once glutamatergic PSDs reached a certain size, they stabilized and GluRIIA was essentially immobilized. In comparison, other postsynaptic proteins showed high turnover equally over all synapses (Rasse et al., 2005). This slow turnover of glutamate receptors is consistent with the view that multiple interactions of glutamate receptors set the core of a transsynaptic interaction matrix. Several lines of genetic and experience-dependent manipulations point towards a rate-limiting role of GluRIIA levels in NMJ synapse formation (Broadie and Bate, 1993; Reiff et al., 2002; Sigrist et al., 2002; Yoshihara et al., 2005). In summary, the available data suggest that incorporation of glutamate receptors might be a key event to allow further expansion of initial postsynaptic assemblies, finally leading to mature PSDs. Thereby, the overall level of glutamate receptors available in the muscle membrane might control the total number of synapses forming per NMJ (Rasse et al., 2005).

Understanding the plasticity processes taking place at glutamatergic synapses has been a focus of attention within cellular neuroscience. Hereby, rapid changes in 
synaptic receptor number were reported to mediate plastic changes of synaptic transmission, often on the time scale of tens of minutes in mammalian preparations (Malinow and Malenka, 2002; Sheng and Kim, 2002; Bredt and Nicoll, 2003; Collingridge et al., 2004). Notably, however, a recent study indicated that the cycling of synaptic glutamate receptors needed 16 hours or more (Adesnik et al., 2005). Similar timing was observed for nicotinic acetylcholine and GABA receptors (Akaaboune et al., 2002; Thomas et al., 2005). Thus, parts of the synaptic glutamate receptor population might be needed to reside stably within the PSD to maintain synapse stability. In fact, only severe receptor deprivation interfered with proper postsynaptic assembly at the $\mathrm{NMJ}$, suggesting that the glutamate receptor level should not fall below a certain critical threshold.

Notably, the extracellular domain of the mammalian AMPA receptor subunit GluR2 has been shown to increase the size and density of spines in hippocampal neurons, and to induce spine formation in GABAergic interneurons normally lacking spines (Passafaro et al., 2003). It will be interesting to see whether these structural roles of glutamate receptors have a common mechanistic denominator.

Different types of synapses differ strongly in the ultrastructural detail of their postsynaptic specializations. Thus, a typical neuron of our brain, acting as a postsynaptic partner for different types of presynaptic inputs, has to establish and maintain different postsynaptic architectures, suggesting the existence of "identity molecules" allowing the self-assembly of such architectures, and potentially a match with membrane cues of the presynaptic partner cell. Obvious candidates for such molecules are the postsynaptic neurotransmitter receptors themselves. This study is consistent with such a view. 


\subsection{Functional fluorophore-tagging of glutamate receptor subunits}

The maturation of postsynaptic densities essentially involves glutamate receptors. Thereby, subunit-specific trafficking of AMPA receptors was shown to play a major role for the activation and the maintenance of synaptic transmission (Shi, 2001). At Drosophila NMJ synapses, the specific incorporation of GluRIIA-type complexes, which was tracked with C-terminally labeled GluRIIA, correlated with the respective growth of single PSDs (Rasse et al., 2005). The role of the second receptor subtype (containing the subunit GluRIIB) in the dynamic PSD maturation process had so far not been addressed.

All GFP fusions of mammalian AMPA receptors were produced by inserting EGFP directly after the signal peptide of the respective glutamate receptor subunit (Shi et al., 1999; Perestenko and Henley, 2003). However, the identical position within GluRIIA and GluRIIB proved to be non-functional. To screen for other possible Nterminal fusion sites, which would ease studying the role of the C-terminal domain, GluRIIA and GluRIIB were subjected to two independent approaches for functional fluorescence-tagging.

Both fusion approaches were deduced from a recent in vitro transposition screen performed for rat GluR1 (Sheridan et al., 2002). The first procedure implied a transformation of the in vitro transposition reaction to Drosophila. The random integration of an EGFP transposon into the genomic transgenes gluRIIA and gluRIIB resulted in numerous insertions within the respective coding region. However, only 8 of 50 (g/uRIIA) and accordingly 9 of 22 (g/uRIIB) clones met the correct reading frame. 42 and respectively 13 clones carried insertions out of frame or within introns. All in frame insertions, which were spread all over the protein (Fig. 29 and Fig. 30), proved to be non-functional (no rescue of the otherwise lethal g/uRIIA $A^{\text {null }} / I B^{\text {null }}$ situation) and showed no detectable fluorescent signals. The negative outcome is likely based on the complex structure and function of non-NMDA glutamate receptor channels (see 2.2), involving subunit-specific interactions and conformational changes upon glutamate binding and ion gating.

Specific interactions within the N-terminal domains of the glutamate receptor subunits contribute to subunit dimerization (Madden, 2002). Therefore, all N-terminal EGFP fusions most likely impede receptor complex formation. Insertions within the ligand binding domain S1/S2 can effect the organization of the glutamate binding pocket, presumably resulting in enhanced ER retention or the abolishment of synaptic transmission (Grunwald and Kaplan, 2003). Furthermore, dimer and tetramer stabilization mediated by the ligand binding domain should as well be impaired (Horning and Mayer, 2004). In one case the EGFP interrupted the 
transmembrane domain M2 destructing the hydrophobic character of the lipid bilayer. Two insertions within GluRIIB resided within the CTD about 50 amino acids from the very C-term (after AA 857 and 861). As demonstrated for GluRIIA, C-terminal truncation of 53 amino acids resulted in severe defects of the postsynaptic structure ( $g l u R I I A^{\Delta C 53}$, see 4.1.7), seemingly due to the disruption of C-terminal interactions to

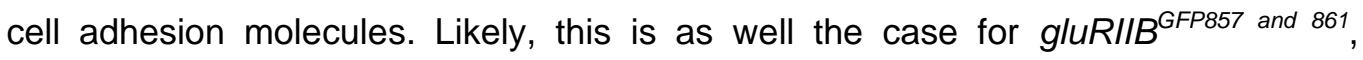
bearing in mind that also receptor transport to the postsynaptic membrane could be inhibited.

In a second approach functional EGFP or ECFP fusion sites within rat GluR1 (Fig. 31) (Sheridan et al., 2002) were assigned to GluRIIA and GluRIIB. Thereby, the transposon based design of the EGFP fusion, including linker sequences and internal restriction sites, was imitated.

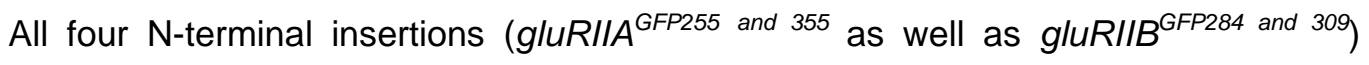
resulted in non-functional proteins without any rescue capability or visible GFP signals. Interestingly, these sites allowed the formation of functional GluR1 homomers (Sheridan et al., 2002) despite the crucial role of the NTD for dimer and receptor complex formation (Madden, 2002). The fact that Drosophila NMJ glutamate receptors are thought to form heterotetramers consisting of four different subunits (Qin et al., 2005) could account for aberrations in the N-terminal 3D structure, which eventually prevent successful GFP fusion.

In contrast, gluRIIB ${ }^{G F P 897}$, with the EGFP insertion site analogue to gluRIIA ${ }^{\text {GFP893 }}$, which was already successfully used for in vivo imaging of glutamate receptor dynamics (Rasse et al., 2005), showed strong and synapse-specific expression. Moreover, neither the physiological properties nor the rescue capability of gluRIIB ${ }^{\text {GFP897 }}$ could be discriminated from untagged gluRIIB. Furthermore, both gluRIIAGFP ${ }^{893}$ and gluRIIBGFP ${ }^{897}$ were shown to mediate previously described longterm plasticity effects at the NMJ (see 4.3.1) (Sigrist et al., 2002). However, although no obvious hints are existing, it cannot be completely excluded that especially Cterminal interactions involved in receptor transport (Malinow and Malenka, 2002) or stabilization of the postsynaptic structure (see 4.1.7) might be effected to a certain degree. In the end, GluRIIB ${ }^{\text {GFP897 }}$ seems to represent a fully functional glutamate receptor subunit qualified for studies on subunit-specific glutamate receptor dynamics and their role in PSD formation and maturation. 


\subsection{Subunit-specific targeting of glutamate receptors organizes PSD formation and maturation}

\subsubsection{Subunit-specific glutamate receptor trafficking}

Memory formation is thought to be accomplished by activity-dependent changes in neurotransmission. Thereby, alterations in synaptic strength as well as the formation of novel synaptic contacts are of importance. The most intensively studied forms of synaptic plasticity are long-term potentiation (LTP) and long-term depression (LTD), for which trafficking of glutamatergic AMPA receptors were shown to play a critical role (Malinow and Malenka, 2002; Song and Huganir, 2002). Here, developing glutamatergic Drosophila NMJ synapses were used to follow the dynamics of both heterotetrameric non-NMDA type glutamate receptor complexes expressed at individual postsynaptic densities. Functional fluorescence-tagging of the two different receptor subtypes, containing either GluRIIA or GluRIIB together with GluRIIC, GluRIID and GluRIIE (Marrus et al., 2004; Chen et al., 2005; Featherstone et al., 2005; Qin et al., 2005), in combination with the recently established in vivo imaging technique (Rasse et al., 2005), enabled the parallel visualization and quantification of both receptor complexes during larval PSD development. As shown before for the endogenous situation (Fig. 34A) (Marrus et al., 2004), tagged GluRIIA and GluRIIB type complexes, overlapped only partially at single PSDs, with especially small PSDs showing heterogeneous receptor content, however mainly dominated by GluRIIA (Fig. 35A, Fig. 37B). During the observation time of $12 \mathrm{~h}$ (at $25^{\circ} \mathrm{C}$ rearing temperature) PSDs initially rich of GluRIIA specifically incorporated GluRIIB and vice versa, striving towards a balanced level of both receptor subtypes (Fig. 37C-F). Thereby, initial PSD size and further PSD growth showed an inverse relationship (Fig. 36). Performing FRAP experiments, it could be shown that the incorporation characteristics of GluRIIA and GluRIIB complexes into PSDs was fundamentally different. GluRIIA recovery was restricted to growing PSDs (Fig. 38) (Rasse et al., 2005). In contrast, GluRIIB showed uniform FRAP over all PSDs (Fig. 38). At the mammalian glutamatergic CNS synapse, similar dynamics for AMPA receptors were shown (Shi, 2001; Barry and Ziff, 2002). Following LTP induction, GluR1/2 complexes were specifically delivered to the postsynaptic plasma membrane to potentiate synaptic transmission. In contrast, the maintenance of synaptic transmission involved the constitutive recycling of GluR2/3 complexes independent of neuronal activity. Several studies showed that currents mediated by GluRIIA are the dominant component of transmission at the Drosophila NMJ synapse whereas GluRIIB currents are low (Petersen et al., 1997; DiAntonio et al., 1999; Reiff et al., 2002; Haghighi et al., 2003). Thereby, specific GluRIIA incorporation into growing 
PSDs would result in long-term strengthening of synaptic transmission similar to the specific integration of GluR1 containing receptors underlying LTP. AMPA receptors at glutamatergic CNS were thought to traffic from intracellular stores to the cell surface within tens of minutes (Liao et al., 1995; Durand et al., 1996; Isaac, 2003). However, a recent study on native AMPA receptors, which are thought to be dimers of GluR1/2 and GluR2/3 dimers (Madden, 2002), suggested a time scale of at least $16 \mathrm{~h}$ for an exchange of the synaptic AMPA receptor population (Adesnik et al., 2005). Similarly, it could be demonstrated that at Drosophila neuromuscular synapses glutamate receptor exchange is slow (maximally $80 \%$ fluorescence recovery after $24 \mathrm{~h}$ ), much slower than the FRAP of various other synaptic proteins (Rasse et al., 2005).

\subsubsection{Factors controlling the differential trafficking of GluRIIA and GluRIIB}

AMPA receptor complexes, being associated with a large protein network, the postsynaptic density, are targeted to the synaptic plasma membrane via subunit specific interactions. Thereby, various, mostly C-terminal interactions and phosphorylation (discussed below) were shown to be responsible for receptor trafficking. The transport of GluR1 containing complexes involves the specific interaction with SAP97, which can be detected already early in the secretory pathway (Sans et al., 2001). Moreover, the protein 4.1N is thought to link GluR1 complexes to the Actin cytoskeleton (Song and Huganir, 2002). GluR2 interactions are predominantly mediated by a PDZ motif at the very C-terminus. The PDZ proteins GRIP, ABP and PICK1 likely serve diverse functions in transport, clustering and endocytosis of GluR2/3 complexes (Malinow and Malenka, 2002). Additionally, NSF interaction to the cytoplasmic tail of GluR2 likely increases receptor surface expression (Luscher et al., 1999) and their resistance to endocytosis (Shi et al., 2001). Recent evidence also suggests that Stargazin is required for the surface expression of AMPA receptors (Chen et al., 2000). The molecular mechanisms underlying glutamate receptor trafficking at the Drosophila NMJ are largely unknown. Here, a fundamentally different incorporation behavior of the two NMJ expressed glutamate receptor complexes was demonstrated. While GluRIIA complexes were apparently immobilized at the postsynaptic membrane, GluRIIB complexes seemed to cycle continuously. Thereby, the C-terminal domain of GluRIIA was involved in the heterogeneous receptor complex incorporation (Fig. 39A,B), restricted only to growing PSDs (Rasse et al., 2005). In contrast, the CTD of GluRIIA was not sufficient to confer GluRIIA type FRAP behavior to GluRIIB (Fig. 39C,D). However, 
no specific C-terminal interactions of GluRIIA or GluRIIB, which could orchestrate this differential incorporation, have been verified so far. Previous studies imply that the two receptor complexes are trafficked via independent pathways (and phosphorylation, see below). While synaptic expression of GluRIIA involves the protein 4.1N homologue Coracle (Chen et al., 2005), the MAGUK protein Dlg specifically alters synaptic GluRIIB levels (Chen and Featherstone, 2005). Furthermore, GluRIIA expression was shown to be controlled by local translation (Sigrist et al., 2000). Future work should shed more light on the numerous factors (more than 50 mutants affecting synaptic GluRIIA levels were presented recently) (Liebl and Featherstone, 2005) organizing receptor transport at the Drosophila NMJ.

\subsubsection{Phosphorylation and receptor trafficking}

The direct C-terminal phosphorylation of glutamate receptors plays an important role for the regulated receptor incorporation during LTD and LTP. Whereas CamKII phosphorylation of S831 on GluR1 is not necessary for AMPA receptor transport (Hayashi et al., 2000), PKA phosphorylation of S845 (Malinow and Malenka, 2002) and PKC phosphorylation of S818 (Boehm et al., 2006) were shown to be needed for synaptic delivery of GluR1 during LTP. During LTD, S845 (Lee et al., 2000) on GluR1 is dephosphorylated. Moreover, LTD induction goes along with PKC phosphorylation of S880 on GluR2, resulting in increased receptor internalization due to the lowered affinity for GRIP and ABP, but not for PICK1 (Chung et al., 2000). Furthermore, PKA phosphorylation of GluR4 was shown to be sufficient for synaptic incorporation of GluR4 containing complexes (Esteban et al., 2003). The Drosophila NMJ glutamate receptor subunits GluRIIA and GluRIIB contain various putative phosphorylation sites within the CTD. While GluRIIA harbors two potential PKA phosphorylation sites at S891 and S897 (see Fig. 28), no such motifs can be found within GluRIIB. Indeed, various studies associated GluRIIA incorporation with the CAMP second messenger pathway (see below) (Davis et al., 1998; Haghighi et al., 2003; Morimoto-Tanifuji et al., 2004). GluRIIB instead contains a PKC motif at the homologue position to S818 of GluR1, which was recently linked to synaptic delivery of GluR1/2 complexes (Boehm et al., 2006).

While at vertebrate glutamatergic CNS synapses $\mathrm{Ca}^{2+}$ influx into the postsynaptic cell occurs through NMDA receptor channels (Barry and Ziff, 2002), NMDA receptors were so far not described at the glutamatergic Drosophila NMJ synapses. Thus, the non-NMDA type glutamate receptors account for the $\mathrm{Ca}^{2+}$ influx (Chang et al., 1994), which activates the calmodulin-dependent CAMP cascade, at last resulting in activation of PKA. Thereby, the conductance of GluRIIB complexes, which show an 
about ten times faster desensitization than GluRIIA containing channels but a comparable single-channel amplitude (DiAntonio et al., 1999; Chen and Featherstone, 2005), is virtually negligible, means probably $\mathrm{Ca}^{2+}$ influx predominantly occurs through GluRIIA complexes (Fig. 44). $\mathrm{Ca}^{2+}$ is intracellularly sensed by Calmodulin, which subsequently activates the adenyl cyclase Rutabaga. Rutabaga converts ATP to CAMP, which in turn activates PKA. PKA is thought to specifically phosphorylate the CTD of GluRIIA but not GluRIIB, thereby controlling GluRIIA incorporation characteristics.

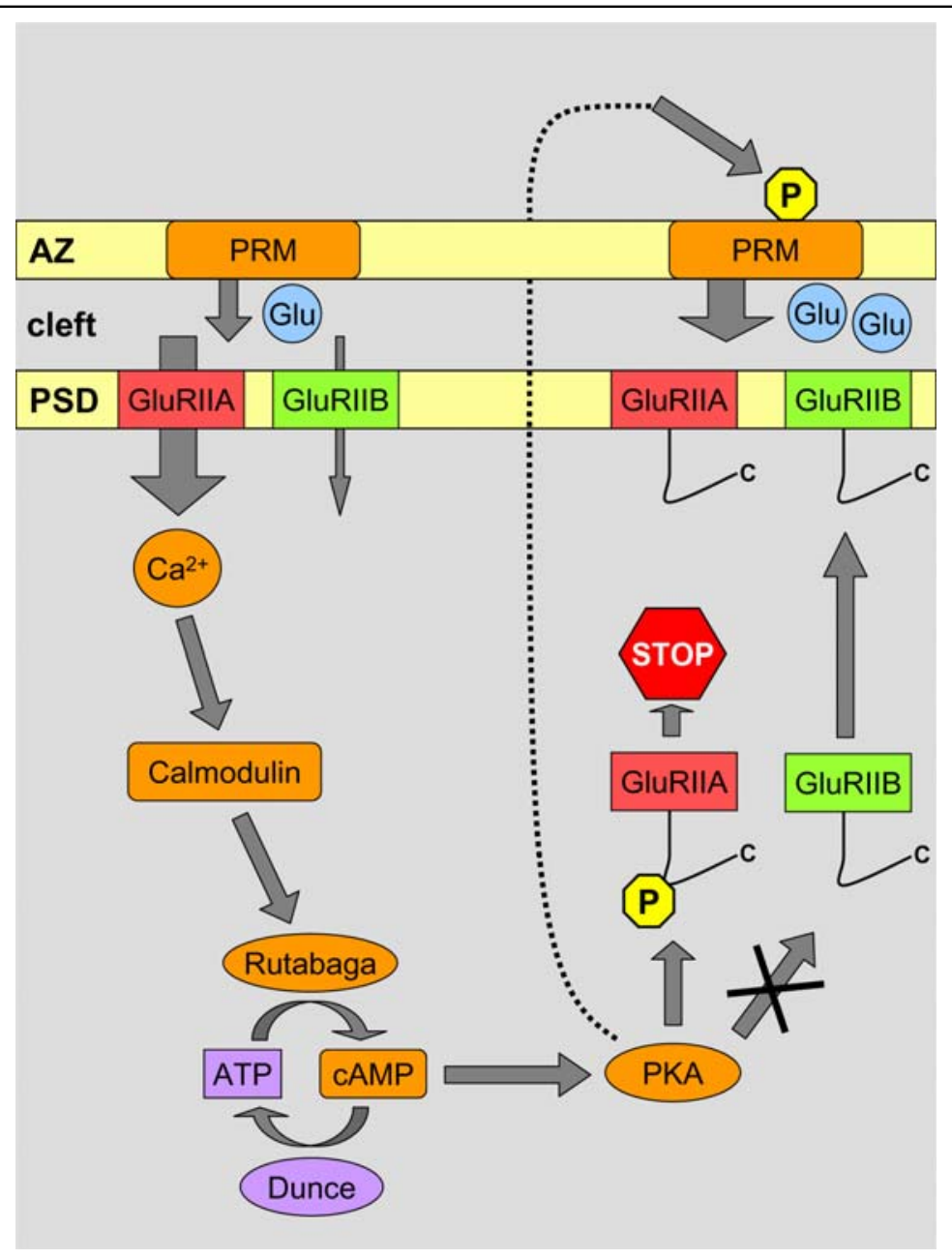

Fig. 53. Model: Differential glutamate receptor incorporation involves the cAMP cascade

GluRIIA complexes show an about 10-fold higher conductivity than GluRIIB and predominantly account for the $\mathrm{Ca}^{2+}$ influx into the postsynaptic muscle cell. $\mathrm{Ca}^{2+}$ is intracellularly sensed by Calmodulin, which activates the adenyl cyclase Rutabaga. Rutabaga activation results in cAMP production, which in turn activates PKA. PKA phosphorylation of the GluRIIA CTD inhibits further incorporation of GluRIIA into mature PSDs. In contrast, GluRIIB is not sensitive to phosphorylation and incorporates uniformly over all PSDs. The retrograde PKA signal regulating presynaptic transmitter release is indicated by the dashed line. Abbreviations denote: PRM presynaptic release machinery, $\mathrm{P}$ - phosphorylation, Glu - glutamate-filled synaptic vesicle. 
Based on the presented model (Fig. 53), PSDs rich of GluRIIA complexes show substantial $\mathrm{Ca}^{2+}$ influx into the postsynaptic muscle cell. This results in high cAMP production, increased PKA activation and thus phosphorylation of GluRIIA, which inhibits further incorporation of GluRIIA complexes. In that way, a maximum conductance value of the whole PSD could be set. In contrast, PSDs rich of GluRIIB complexes show very low $\mathrm{Ca}^{2+}$ influx into the postsynaptic muscle cell, at last hardly resulting in GluRIIA phosphorylation. Thus, incorporation of (dephosphorylated) GluRIIA is favored, finally leading to a balanced level of both receptor subtypes at individual PSDs (Fig. 39C-F). A previous study on postsynaptic PKA supports this interpretation (Davis et al., 1998). Postsynaptic expression of a constitutively active form of PKA resulted in reduced quantal size going along with a retrograde increase in presynaptic transmitter release. Contrary to it, inhibiting PKA activity led to an increase in quantal size. In both cases the PKA-dependent modulation of the quantal size required the presence of GluRIIA, clearly indicating PKA regulation of GluRIIA trafficking. The retrograde modulation of synaptic transmission was also shown to be controlled by postsynaptic CamKII (Haghighi et al., 2003). Thereby, strong $\mathrm{Ca}^{2+}$ influx mediated by GluRIIA complexes results in CamKII autophosphorylation and activation which impedes the retrograde increase in presynaptic activity. A recent study implies that GluRIIA levels might be as well regulated by CamKII (MorimotoTanifuji et al., 2004). Postsynaptic CamKII overexpression on $1^{\text {st }}$ instar NMJs resulted in slightly reduced GluRIIA immunoreactivity. Moreover, both pre- and postsynaptic expression of a constitutively active form of the alpha subunit of the heterotrimetric G protein (Gas), which positively regulates the activity of Rutabaga, resulted in strongly decreased synaptic GluRIIA levels (Renden and Broadie, 2003). However, the same phenomenon could be observed after genetic elimination of Gas, possibly mediated by compensatory activation of the cAMP cascade.

\subsubsection{Activity-dependent PSD regulation - blocked neurotransmission}

Synaptic vesicle release is supposed to trigger $\mathrm{Ca}^{2+}$ influx into the postsynaptic muscle cell (Guerrero et al., 2005), which seems to involve into glutamate receptor trafficking at the PSD. In the absence of neurotransmission the initial formation of neuronal circuits and synapses persists (Verhage et al., 2000; Baines et al., 2001; Varoqueaux et al., 2002; Heeroma et al., 2003). At the vertebrate NMJ, a cutback of activity resulted in increased nerve-terminal sprouting with varying effects on the end-plate length (Wilson and Deschenes, 2005). Moreover, fast internalization of acetylcholine receptors could be observed when neurotransmission was blocked (Akaaboune et al., 1999). Here, glutamatergic synapses at the Drosophila NMJ were 
studied under activity suppression by mosaic TNT expression, which largely abolishes evoked junctional responses (Sweeney et al., 1995). In vivo imaging (Fig. 46A-F) and immunostainings (Fig. 48) showed that GluRIIA accumulation was increased, predominantly at small PSDs, while GluRIIB remained unaffected. Consistently, no reduction in synaptic glutamate receptor levels could be observed (Fig. 49E). Moreover, the average PSD size (determined via glutamate receptor abundance) was significantly increased after activity blockage (Fig. 48C).

A working model for the activity suppressed situation is provided in Fig. 54. The initial formation of PSDs subjected to activity blockage, is primarily dominated by GluRIIA accumulation. As nerve-evoked currents are absent, only residual miniature currents and non-vesicular glutamate release can account for some minimal $\mathrm{Ca}^{2+}$ influx. However, non-vesicular glutamate release, which was shown to regulate glutamate receptor levels and PSD size (Featherstone et al., 2002), had no obvious

effects in this assay. Based on the above presented model (Fig. 53), $\mathrm{Ca}^{2+}$ influx is required for the activation of the cAMP cascade and receptor phosphorylation. Therefore, in the activity suppressed background GluRIIA would, as observed, no longer be restricted from incorporation into mature PSDs and show more uniform FRAP (Fig. 39E, Fig. 46H). As the synaptic transmission strength cannot reach mature status, receptors likely keep on incorporating, reflected in the increased PSD size (Fig. 48C). Similarly, in mutants for the adenyl cyclase Rutabaga (Renger et al., 2000; Shayan and Atwood, 2000) the PSD size was strongly increased, further indicating a role of PKA phosphorylation for the limitation of PSD growth and receptor incorporation.

\subsubsection{Activity-dependent PSD regulation - enhanced neurotrans- mission}

Previously enormous morphological outgrowth of larval NMJs going along with potentiation of synaptic transmission was linked with an increase in the breeding temperature (Sigrist et al., 2003; Zhong and Wu, 2004). Similarly, activity elevation was shown to increase the nerve terminal and postsynaptic area at vertebrate NMJs (Wilson and Deschenes, 2005). Here, shifting the rearing temperature from $25^{\circ} \mathrm{C}$ to $29^{\circ} \mathrm{C}$ resulted in a dramatic reduction in the synaptic expression of both GluRIIA and GluRIIB complexes (Fig. 49A-C). In contrast, PSDs of activity suppressed larvae raised at $29^{\circ} \mathrm{C}$ showed no drop in receptor content (Fig. 49G-J). Interestingly, miniature currents measured at $29^{\circ} \mathrm{C}$ animals are not significantly altered, whereas both evoked currents and quantal content are increased (Sigrist et al., 2003). Consequently, a reduced number of glutamate receptors must account for 
unchanged transmission strength at an individual synapse (Fig. 54), calling for a role of posttranslational modifications on channel function. Notably, GluRIIA levels were previously shown to be elevated (Sigrist et al., 2003) while here an enormous reduction at $29^{\circ} \mathrm{C}$ was observed. The answer to this problem could be different antiGluRIIA antibodies. While the initially used antibody was generated against a Cterminal sequence of GluRIIA (likely in a dephosphorylated state; S. Sigrist, personal communication), the antibody used in this study was developed based on an extracellular epitope, which is independent of C-terminal phosphorylation.

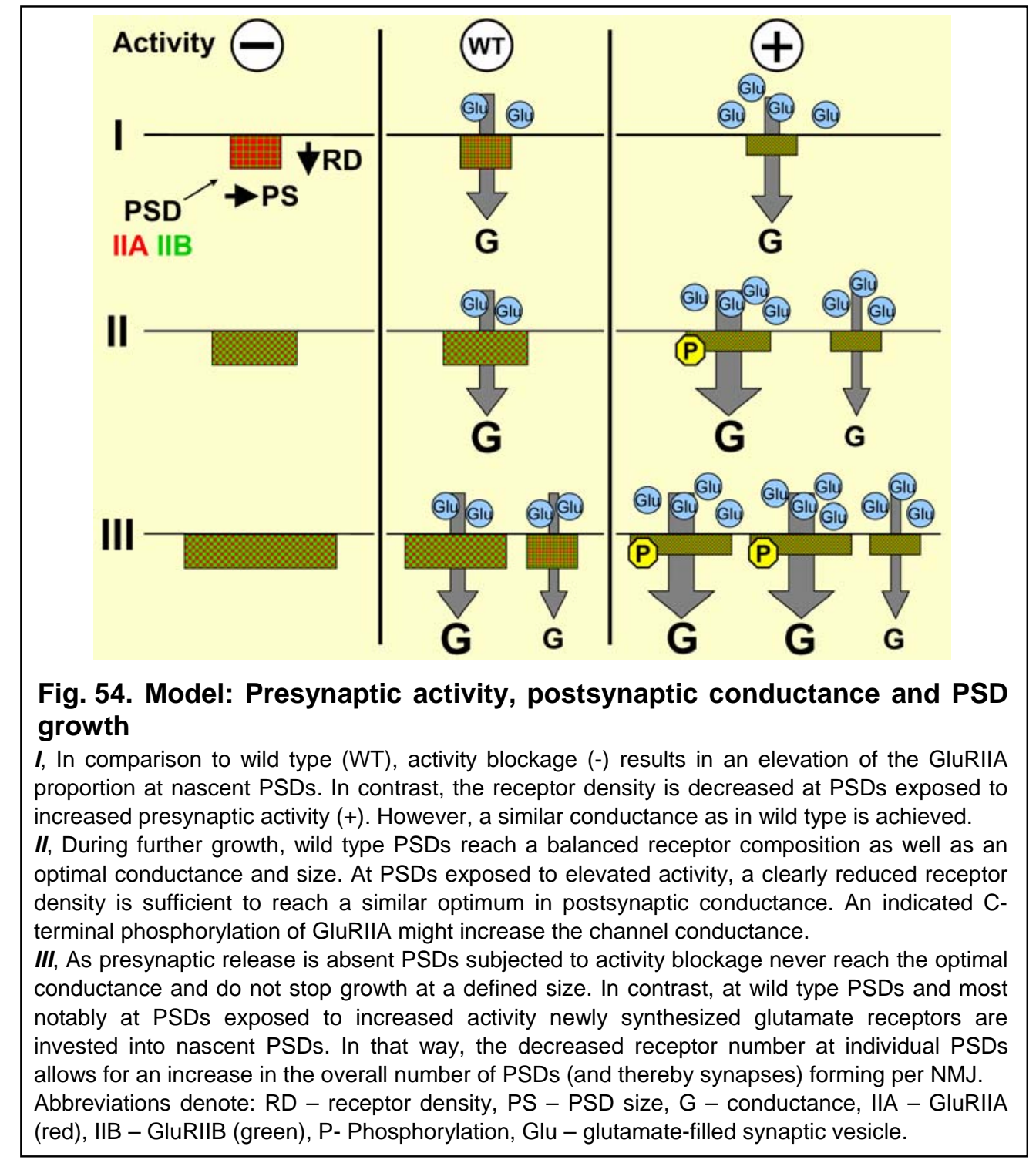

Besides its role in receptor trafficking, phosphorylation involves as well into the regulation of the ion channel function. Phosphorylation of GluR1 at S831 and S845 during LTP was shown to potentiate the channel function, means either increasing the channel open probability or the conductance (Song and Huganir, 2002). While a 
modulation of the channel conductance was mediated by PKA phosphorylation of S845 (Banke et al., 2000), the open probability can be modified by CamKII phosphorylation on S831 (Derkach et al., 1999). As mentioned above, GluRIIA harbors two putative PKA phosphorylation sites, while neither GluRIIA nor GluRIIB contain a homologous motif for CamKII phosphorylation (see Fig. 28). Accordingly, one possible mechanism could be that the two PKA sites serve to fulfill different roles. While phosphorylation of the first site might inhibit further GluRIIA incorporation into mature PSDs (Fig. 53), the second PKA site might involve into the modulation of the channel conductance (Fig. 54). However, both PKA sites are not necessary for the principal targeting of GluRIIA complexes to the postsynaptic membrane. After truncation of the last 17 amino acids of the GluRIIA C-terminal domain (gluRIIA ${ }^{\Delta C 17}$ ) and thereby eliminating both putative PKA sites, synaptic glutamate receptor levels appeared unaltered compared to controls (see Table 3 ).

Additional in vivo experiments are clearly required to certify the ultimate role of receptor phosphorylation. Basis for these future studies would be a lumenal fluorophore fusion, which would allow studying C-terminal modifications with in vivo imaging. However, several attempts to produce functional lumenal GFP fusions of GluRIIA were to no avail (see 4.2).

\subsubsection{Physiological relevance of GluRIIA and GluRIIB complexes}

Several previous studies have addressed the principal physiological properties of the two NMJ expressed glutamate receptor complexes. Thereby, as mentioned above, GluRIIA was shown to be predominantly responsible for the overall postsynaptic conductance (Petersen et al., 1997; DiAntonio et al., 1999; Reiff et al., 2002; Sigrist et al., 2002). Whereas the positive retrograde control of presynaptic release by PKA required the presence of GluRIIA (Davis et al., 1998), the negative control by CamKII was demonstrated for the gluRIIA mutant (Haghighi et al., 2003). However, both mechanisms are based on $\mathrm{Ca}^{2+}$ influx into the postsynaptic compartment, which is strongly dependent on GluRIIA levels. Notably, both gluRIIA and gluRIIB are redundant for viability (Petersen et al., 1997; DiAntonio et al., 1999; Marrus et al., 2004; Qin et al., 2005). Thus, the question remains what physiological relevance GluRIIB complexes have?

FRAP experiments in the respective absence of the other receptor subtype uncovered that the specific incorporation pattern of GluRIIA persisted (Fig. 41A) while GluRIIB kept on continuously recycling (Fig. 41B), obviously independent of the PSD status. However, differences in the development of the PSD sizes could be uncovered (Fig. 43). In the absence of GluRIIA, PSD growth was slower but 
seemingly less limited. Consistently, an atypical size distribution with a majority of small PSDs was observed. PSDs solely expressing GluRIIA grew faster, matching the recent finding that GluRIIA incorporation is directly correlated to PSD growth (Rasse et al., 2005). Moreover, mere GluRIIA presence should support a faster convergence to the optimal conductance, thereby allocating more receptor complexes for the establishment of novel synaptic contacts (Fig. 55).

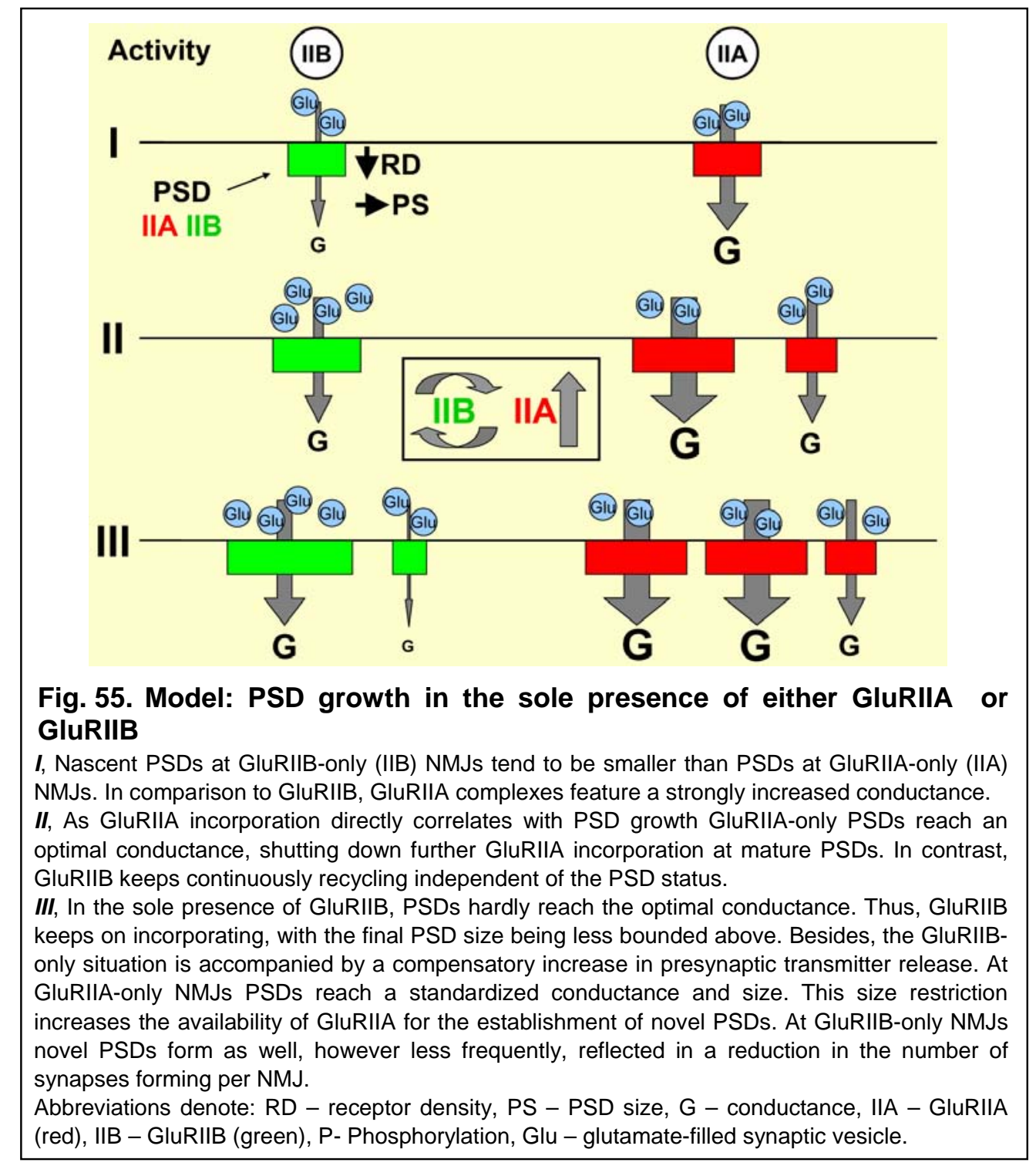

Indeed, overexpression of GluRIIA (Sigrist et al., 2002) or absence of GluRIIB (Fig. 34) was shown to increase the number of synapses forming per NMJ. Similarly, an elevation of the raising temperature dramatically increased the bouton number and the overall NMJ transmission strength (Sigrist et al., 2003; Zhong and Wu, 2004). Here, it could be shown that in the absence of GluRIIB, means in an already potentiated background, more new PSDs formed after further activity enhancement 
(rearing at $29^{\circ} \mathrm{C}$, Fig. 50B). In contrast, activity suppression resulted in a reduction of synaptic contacts per NMJ (Fig. 50A). Thereby, a regulated interplay of glutamate receptor conductance and retrograde modulation of presynaptic release seems to define the PSD receptor composition, the PSD size and the number of synapses forming per NMJ. While for the most part GluRIIA complexes ensure the synaptic transmission, GluRIIB complexes might be responsible for the fine tuning of the preand postsynaptic communication. The faster desensitization and likely reactivation of GluRIIB receptors could be valuable during persistent high-frequency motor activity to maintain a solid level of muscle action. 


\section{References}

\subsection{Scientific articles and monographies}

Adesnik H, Nicoll RA, England PM (2005) Photoinactivation of native AMPA receptors reveals their real-time trafficking. Neuron 48:977-985.

Ahmari SE, Buchanan J, Smith SJ (2000) Assembly of presynaptic active zones from cytoplasmic transport packets. Nat Neurosci 3:445-451.

Akaaboune M, Culican SM, Turney SG, Lichtman JW (1999) Rapid and reversible effects of activity on acetylcholine receptor density at the neuromuscular junction in vivo. Science 286:503-507.

Akaaboune M, Grady RM, Turney S, Sanes JR, Lichtman JW (2002) Neurotransmitter receptor dynamics studied in vivo by reversible photounbinding of fluorescent ligands. Neuron 34:865-876.

Albin SD, Davis GW (2004) Coordinating structural and functional synapse development: postsynaptic p21-activated kinase independently specifies glutamate receptor abundance and postsynaptic morphology. J Neurosci 24:6871-6879.

Aronoff R, Mellem JE, Maricq AV, Sprengel R, Seeburg PH (2004) Neuronal toxicity in Caenorhabditis elegans from an editing site mutant in glutamate receptor channels. J Neurosci 24:8135-8140.

Ashby MC, Ibaraki K, Henley JM (2004) It's green outside: tracking cell surface proteins with $\mathrm{pH}$-sensitive GFP. Trends Neurosci 27:257-261.

Atwood HL (2006) Neuroscience. Gatekeeper at the synapse. Science 312:10081009.

Atwood HL, Govind CK, Wu CF (1993) Differential ultrastructure of synaptic terminals on ventral longitudinal abdominal muscles in Drosophila larvae. J Neurobiol 24:1008-1024.

Ayalon G, Stern-Bach Y (2001) Functional assembly of AMPA and kainate receptors is mediated by several discrete protein-protein interactions. Neuron 31:103113.

Baines RA, Uhler JP, Thompson A, Sweeney ST, Bate M (2001) Altered electrical properties in Drosophila neurons developing without synaptic transmission. J Neurosci 21:1523-1531.

Banke TG, Bowie D, Lee H, Huganir RL, Schousboe A, Traynelis SF (2000) Control of GluR1 AMPA receptor function by cAMP-dependent protein kinase. J Neurosci 20:89-102.

Barry MF, Ziff EB (2002) Receptor trafficking and the plasticity of excitatory synapses. Curr Opin Neurobiol 12:279-286.

Bate M, Landgraf M, Ruiz Gomez Bate M (1999) Development of larval body wall muscles. Int Rev Neurobiol 43:25-44.

Bliss TV, Lomo T (1973) Long-lasting potentiation of synaptic transmission in the dentate area of the anaesthetized rabbit following stimulation of the perforant path. J Physiol 232:331-356.

Boehm J, Kang MG, Johnson RC, Esteban J, Huganir RL, Malinow R (2006) Synaptic incorporation of AMPA receptors during LTP is controlled by a PKC phosphorylation site on GluR1. Neuron 51:213-225.

Borgdorff AJ, Choquet D (2002) Regulation of AMPA receptor lateral movements. Nature 417:649-653.

Brand AH, Perrimon N (1993) Targeted gene expression as a means of altering cell fates and generating dominant phenotypes. Development 118:401-415.

Bredt DS, Nicoll RA (2003) AMPA receptor trafficking at excitatory synapses. Neuron 40:361-379.

Bresler T, Shapira M, Boeckers T, Dresbach T, Futter M, Garner CC, Rosenblum K, Gundelfinger ED, Ziv NE (2004) Postsynaptic density assembly is 
fundamentally different from presynaptic active zone assembly. J Neurosci 24:1507-1520.

Broadie K, Bate M (1993) Innervation directs receptor synthesis and localization in Drosophila embryo synaptogenesis. Nature 361:350-353.

Broadie KS, Bate M (1993) Development of the embryonic neuromuscular synapse of Drosophila melanogaster. J Neurosci 13:144-166.

Campbell RE, Tour O, Palmer AE, Steinbach PA, Baird GS, Zacharias DA, Tsien RY (2002) A monomeric red fluorescent protein. Proc Natl Acad Sci U S A 99:7877-7882.

Carroll RC, Lissin DV, von Zastrow M, Nicoll RA, Malenka RC (1999) Rapid redistribution of glutamate receptors contributes to long-term depression in hippocampal cultures. Nat Neurosci 2:454-460.

Castiglioni MC (1951) [Distribution of pigments in the eye of alleles of white and their compounds in Drosophila melanogaster.]. Sci Genet 4:57-60.

Chang $\mathrm{H}$, Ciani S, Kidokoro $\mathrm{Y}$ (1994) Ion permeation properties of the glutamate receptor channel in cultured embryonic Drosophila myotubes. J Physiol 476:116.

Chen K, Featherstone DE (2005) Discs-large (DLG) is clustered by presynaptic innervation and regulates postsynaptic glutamate receptor subunit composition in Drosophila. BMC Biol 3:1.

Chen K, Merino C, Sigrist SJ, Featherstone DE (2005) The 4.1 protein coracle mediates subunit-selective anchoring of Drosophila glutamate receptors to the postsynaptic actin cytoskeleton. J Neurosci 25:6667-6675.

Chen L, Chetkovich DM, Petralia RS, Sweeney NT, Kawasaki Y, Wenthold RJ, Bredt DS, Nicoll RA (2000) Stargazin regulates synaptic targeting of AMPA receptors by two distinct mechanisms. Nature 408:936-943.

Cheung US, Shayan AJ, Boulianne GL, Atwood HL (1999) Drosophila larval neuromuscular junction's responses to reduction of cAMP in the nervous system. J Neurobiol 40:1-13.

Chklovskii DB, Mel BW, Svoboda K (2004) Cortical rewiring and information storage. Nature 431:782-788.

Chung HJ, Xia J, Scannevin RH, Zhang X, Huganir RL (2000) Phosphorylation of the AMPA receptor subunit GluR2 differentially regulates its interaction with PDZ domain-containing proteins. J Neurosci 20:7258-7267.

Collingridge GL, Isaac JT, Wang YT (2004) Receptor trafficking and synaptic plasticity. Nat Rev Neurosci 5:952-962.

Davis GW, DiAntonio A, Petersen SA, Goodman CS (1998) Postsynaptic PKA controls quantal size and reveals a retrograde signal that regulates presynaptic transmitter release in Drosophila. Neuron 20:305-315.

Davis RL, Kauvar LM (1984) Drosophila cyclic nucleotide phosphodiesterases. Adv Cyclic Nucleotide Protein Phosphorylation Res 16:393-402.

Dean C, Dresbach T (2006) Neuroligins and neurexins: linking cell adhesion, synapse formation and cognitive function. Trends Neurosci 29:21-29.

DeChiara TM, Bowen DC, Valenzuela DM, Simmons MV, Poueymirou WT, Thomas S, Kinetz E, Compton DL, Rojas E, Park JS, Smith C, DiStefano PS, Glass DJ, Burden SJ, Yancopoulos GD (1996) The receptor tyrosine kinase MuSK is required for neuromuscular junction formation in vivo. Cell 85:501-512.

Derkach V, Barria A, Soderling TR (1999) Ca2+/calmodulin-kinase II enhances channel conductance of alpha-amino-3-hydroxy-5-methyl-4isoxazolepropionate type glutamate receptors. Proc Natl Acad Sci U S A 96:3269-3274.

DiAntonio A, Petersen SA, Heckmann M, Goodman CS (1999) Glutamate receptor expression regulates quantal size and quantal content at the Drosophila neuromuscular junction. J Neurosci 19:3023-3032. 
Dornan S, Jackson AP, Gay NJ (1997) Alpha-adaptin, a marker for endocytosis, is expressed in complex patterns during Drosophila development. Mol Biol Cell 8:1391-1403.

Dudai Y, Zvi S (1985) Multiple defects in the activity of adenylate cyclase from the Drosophila memory mutant rutabaga. J Neurochem 45:355-364.

Dudai Y, Jan YN, Byers D, Quinn WG, Benzer S (1976) dunce, a mutant of Drosophila deficient in learning. Proc Natl Acad Sci U S A 73:1684-1688.

Durand GM, Kovalchuk Y, Konnerth A (1996) Long-term potentiation and functional synapse induction in developing hippocampus. Nature 381:71-75.

Echalier G (1997) Drosophila: Cells in Culture, 1st Edition. New York: Academic Press.

Esteban JA, Shi SH, Wilson C, Nuriya M, Huganir RL, Malinow R (2003) PKA phosphorylation of AMPA receptor subunits controls synaptic trafficking underlying plasticity. Nat Neurosci 6:136-143.

Featherstone D, Broadie K (2002) Response: meaningless minis? Trends Neurosci 25:386-387.

Featherstone DE, Broadie K (2000) Surprises from Drosophila: genetic mechanisms of synaptic development and plasticity. Brain Res Bull 53:501-511.

Featherstone DE, Rushton E, Broadie K (2002) Developmental regulation of glutamate receptor field size by nonvesicular glutamate release. Nat Neurosci 5:141-146.

Featherstone DE, Rushton EM, Hilderbrand-Chae M, Phillips AM, Jackson FR, Broadie K (2000) Presynaptic glutamic acid decarboxylase is required for induction of the postsynaptic receptor field at a glutamatergic synapse. Neuron 27:71-84.

Featherstone DE, Rushton E, Rohrbough J, Liebl F, Karr J, Sheng Q, Rodesch CK, Broadie K (2005) An essential Drosophila glutamate receptor subunit that functions in both central neuropil and neuromuscular junction. J Neurosci 25:3199-3208.

Feng Y, Ueda A, Wu CF (2004) A modified minimal hemolymph-like solution, HL3.1, for physiological recordings at the neuromuscular junctions of normal and mutant Drosophila larvae. J Neurogenet 18:377-402.

Fiala JC, Allwardt B, Harris KM (2002) Dendritic spines do not split during hippocampal LTP or maturation. Nat Neurosci 5:297-298.

Gautam M, Noakes PG, Mudd J, Nichol M, Chu GC, Sanes JR, Merlie JP (1995) Failure of postsynaptic specialization to develop at neuromuscular junctions of rapsyn-deficient mice. Nature 377:232-236.

Gorczyca M, Popova E, Jia XX, Budnik V (1999) The gene $\bmod (\operatorname{mdg} 4)$ affects synapse specificity and structure in Drosophila. J Neurobiol 39:447-460.

Grunwald ME, Kaplan JM (2003) Mutations in the ligand-binding and pore domains control exit of glutamate receptors from the endoplasmic reticulum in C. elegans. Neuropharmacology 45:768-776.

Guerrero G, Reiff DF, Agarwal G, Ball RW, Borst A, Goodman CS, Isacoff EY (2005) Heterogeneity in synaptic transmission along a Drosophila larval motor axon. Nat Neurosci 8:1188-1196.

Gundelfinger ED, Kessels MM, Qualmann B (2003) Temporal and spatial coordination of exocytosis and endocytosis. Nat Rev Mol Cell Biol 4:127-139.

Haghighi AP, McCabe BD, Fetter RD, Palmer JE, Hom S, Goodman CS (2003) Retrograde control of synaptic transmission by postsynaptic CaMKII at the Drosophila neuromuscular junction. Neuron 39:255-267.

Harris WA (1980) The effects of eliminating impulse activity on the development of the retinotectal projection in salamanders. J Comp Neurol 194:303-317. 
Hayashi Y, Shi SH, Esteban JA, Piccini A, Poncer JC, Malinow R (2000) Driving AMPA receptors into synapses by LTP and CaMKII: requirement for GluR1 and PDZ domain interaction. Science 287:2262-2267.

Hebb D (1949) The organization of behaviour. New York, NY: Wiley.

Heeroma JH, Plomp JJ, Roubos EW, Verhage M (2003) Development of the mouse neuromuscular junction in the absence of regulated secretion. Neuroscience 120:733-744.

Horn C, Wimmer EA (2000) A versatile vector set for animal transgenesis. Dev Genes Evol 210:630-637.

Horning MS, Mayer ML (2004) Regulation of AMPA receptor gating by ligand binding core dimers. Neuron 41:379-388.

Hughes BW, Kusner LL, Kaminski HJ (2006) Molecular architecture of the neuromuscular junction. Muscle Nerve 33:445-461.

Isaac JT (2003) Postsynaptic silent synapses: evidence and mechanisms. Neuropharmacology 45:450-460.

Jan LY, Jan YN (1976) Properties of the larval neuromuscular junction in Drosophila melanogaster. J Physiol 262:189-214.

Jonas P, Burnashev N (1995) Molecular mechanisms controlling calcium entry through AMPA-type glutamate receptor channels. Neuron 15:987-990.

Kandel E, Schwartz J, Jessell T (1991) Principles of neural science, 3rd Edition. East Norwalk, CT: Appleton \& Lange.

Kasai H, Matsuzaki M, Noguchi J, Yasumatsu N, Nakahara H (2003) Structurestability-function relationships of dendritic spines. Trends Neurosci 26:360-368.

Kask K, Zamanillo D, Rozov A, Burnashev N, Sprengel R, Seeburg PH (1998) The AMPA receptor subunit GluR-B in its Q/R site-unedited form is not essential for brain development and function. Proc Natl Acad Sci U S A 95:13777-13782.

Keshishian H, Broadie K, Chiba A, Bate M (1996) The drosophila neuromuscular junction: a model system for studying synaptic development and function. Annu Rev Neurosci 19:545-575.

Kim E, Sheng M (2004) PDZ domain proteins of synapses. Nat Rev Neurosci 5:771781.

Kim JH, Udo H, Li HL, Youn TY, Chen M, Kandel ER, Bailey CH (2003) Presynaptic activation of silent synapses and growth of new synapses contribute to intermediate and long-term facilitation in Aplysia. Neuron 40:151-165.

Kim S, Ko J, Shin H, Lee JR, Lim C, Han JH, Altrock WD, Garner CC, Gundelfinger ED, Premont RT, Kaang BK, Kim E (2003) The GIT family of proteins forms multimers and associates with the presynaptic cytomatrix protein Piccolo. J Biol Chem 278:6291-6300.

Kittel RJ, Wichmann C, Rasse TM, Fouquet W, Schmidt M, Schmid A, Wagh DA, Pawlu C, Kellner RR, Willig KI, Hell SW, Buchner E, Heckmann M, Sigrist SJ (2006) Bruchpilot promotes active zone assembly, Ca2+ channel clustering, and vesicle release. Science 312:1051-1054.

Koenig JH, Saito K, Ikeda K (1983) Reversible control of synaptic transmission in a single gene mutant of Drosophila melanogaster. J Cell Biol 96:1517-1522.

Koh YH, Gramates LS, Budnik V (2000) Drosophila larval neuromuscular junction: molecular components and mechanisms underlying synaptic plasticity. Microsc Res Tech 49:14-25.

Kuromi H, Kidokoro Y (2002) Selective replenishment of two vesicle pools depends on the source of $\mathrm{Ca} 2+$ at the Drosophila synapse. Neuron 35:333-343.

Landgraf M, Thor S (2006) Development of Drosophila motoneurons: specification and morphology. Semin Cell Dev Biol 17:3-11.

Lee HK, Barbarosie M, Kameyama K, Bear MF, Huganir RL (2000) Regulation of distinct AMPA receptor phosphorylation sites during bidirectional synaptic plasticity. Nature 405:955-959. 
Liao D, Hessler NA, Malinow R (1995) Activation of postsynaptically silent synapses during pairing-induced LTP in CA1 region of hippocampal slice. Nature 375:400-404.

Liebl FL, Featherstone DE (2005) Genes involved in Drosophila glutamate receptor expression and localization. BMC Neurosci 6:44.

Livingstone MS (1985) Genetic dissection of Drosophila adenylate cyclase. Proc Natl Acad Sci U S A 82:5992-5996.

Loughney K, Kreber R, Ganetzky B (1989) Molecular analysis of the para locus, a sodium channel gene in Drosophila. Cell 58:1143-1154.

Luscher C, Xia H, Beattie EC, Carroll RC, von Zastrow M, Malenka RC, Nicoll RA (1999) Role of AMPA receptor cycling in synaptic transmission and plasticity. Neuron 24:649-658.

Madden DR (2002) The structure and function of glutamate receptor ion channels. Nat Rev Neurosci 3:91-101.

Malinow R, Malenka RC (2002) AMPA receptor trafficking and synaptic plasticity. Annu Rev Neurosci 25:103-126.

Marangi PA, Forsayeth JR, Mittaud P, Erb-Vogtli S, Blake DJ, Moransard M, Sander A, Fuhrer C (2001) Acetylcholine receptors are required for agrin-induced clustering of postsynaptic proteins. Embo J 20:7060-7073.

Marrus SB, DiAntonio A (2004) Preferential localization of glutamate receptors opposite sites of high presynaptic release. Curr Biol 14:924-931.

Marrus SB, Portman SL, Allen MJ, Moffat KG, DiAntonio A (2004) Differential localization of glutamate receptor subunits at the Drosophila neuromuscular junction. J Neurosci 24:1406-1415.

Matus A (2005) Growth of dendritic spines: a continuing story. Curr Opin Neurobiol 15:67-72.

Mayer ML (2005) Crystal structures of the GluR5 and GluR6 ligand binding cores: molecular mechanisms underlying kainate receptor selectivity. Neuron 45:539552.

McGee AW, Bredt DS (2003) Assembly and plasticity of the glutamatergic postsynaptic specialization. Curr Opin Neurobiol 13:111-118.

Misgeld T, Burgess RW, Lewis RM, Cunningham JM, Lichtman JW, Sanes JR (2002) Roles of neurotransmitter in synapse formation: development of neuromuscular junctions lacking choline acetyltransferase. Neuron 36:635-648.

Missias AC, Mudd J, Cunningham JM, Steinbach JH, Merlie JP, Sanes JR (1997) Deficient development and maintenance of postsynaptic specializations in mutant mice lacking an 'adult' acetylcholine receptor subunit. Development 124:5075-5086.

Morimoto-Tanifuji T, Kazama H, Nose A (2004) Developmental stage-dependent modulation of synapses by postsynaptic expression of activated calcium/calmodulin-dependent protein kinase II. Neuroscience 128:797-806.

Murthy VN, De Camilli P (2003) Cell biology of the presynaptic terminal. Annu Rev Neurosci 26:701-728.

Nikonenko I, Jourdain P, Alberi S, Toni N, Muller D (2002) Activity-induced changes of spine morphology. Hippocampus 12:585-591.

Nishimune A, Isaac JT, Molnar E, Noel J, Nash SR, Tagaya M, Collingridge GL, Nakanishi S, Henley JM (1998) NSF binding to GluR2 regulates synaptic transmission. Neuron 21:87-97.

Ohtsuka T, Takao-Rikitsu E, Inoue E, Inoue M, Takeuchi M, Matsubara K, DeguchiTawarada M, Satoh K, Morimoto K, Nakanishi H, Takai Y (2002) Cast: a novel protein of the cytomatrix at the active zone of synapses that forms a ternary complex with RIM1 and munc13-1. J Cell Biol 158:577-590. 
Ono F, Higashijima S, Shcherbatko A, Fetcho JR, Brehm P (2001) Paralytic zebrafish lacking acetylcholine receptors fail to localize rapsyn clusters to the synapse. J Neurosci 21:5439-5448.

Osten P, Stern-Bach Y (2006) Learning from stargazin: the mouse, the phenotype and the unexpected. Curr Opin Neurobiol.

Packard M, Mathew D, Budnik V (2003) Wnts and TGF beta in synaptogenesis: old friends signalling at new places. Nat Rev Neurosci 4:113-120.

Parnas D, Haghighi AP, Fetter RD, Kim SW, Goodman CS (2001) Regulation of postsynaptic structure and protein localization by the Rho-type guanine nucleotide exchange factor dPix. Neuron 32:415-424.

Partin KM (2001) Domain interactions regulating ampa receptor desensitization. J Neurosci 21:1939-1948.

Passafaro M, Nakagawa T, Sala C, Sheng M (2003) Induction of dendritic spines by an extracellular domain of AMPA receptor subunit GluR2. Nature 424:677-681.

Perestenko PV, Henley JM (2003) Characterization of the intracellular transport of GluR1 and GluR2 alpha-amino-3-hydroxy-5-methyl-4-isoxazole propionic acid receptor subunits in hippocampal neurons. J Biol Chem 278:43525-43532.

Petersen SA, Fetter RD, Noordermeer JN, Goodman CS, DiAntonio A (1997) Genetic analysis of glutamate receptors in Drosophila reveals a retrograde signal regulating presynaptic transmitter release. Neuron 19:1237-1248.

Prokop A (1999) Integrating bits and pieces: synapse structure and formation in Drosophila embryos. Cell Tissue Res 297:169-186.

Prokop A, Meinertzhagen IA (2006) Development and structure of synaptic contacts in Drosophila. Semin Cell Dev Biol 17:20-30.

Prokop A, Landgraf M, Rushton E, Broadie K, Bate M (1996) Presynaptic development at the Drosophila neuromuscular junction: assembly and localization of presynaptic active zones. Neuron 17:617-626.

Purves D, Augustine G, Fitzpatrick D, Katz L, LaMantia A, McNamara J, Williams S (2001) Neuroscience, 2nd Edition. Sunderland, MA: Sinauer Associates.

Qin G, Schwarz T, Kittel RJ, Schmid A, Rasse TM, Kappei D, Ponimaskin E, Heckmann M, Sigrist SJ (2005) Four different subunits are essential for expressing the synaptic glutamate receptor at neuromuscular junctions of Drosophila. J Neurosci 25:3209-3218.

Rasse TM, Fouquet W, Schmid A, Kittel RJ, Mertel S, Sigrist CB, Schmidt M, Guzman A, Merino C, Qin G, Quentin C, Madeo FF, Heckmann M, Sigrist SJ (2005) Glutamate receptor dynamics organizing synapse formation in vivo. Nat Neurosci 8:898-905.

Reiff DF, Thiel PR, Schuster CM (2002) Differential regulation of active zone density during long-term strengthening of Drosophila neuromuscular junctions. J Neurosci 22:9399-9409.

Renden RB, Broadie K (2003) Mutation and activation of Galpha s similarly alters pre- and postsynaptic mechanisms modulating neurotransmission. J Neurophysiol 89:2620-2638.

Renger JJ, Ueda A, Atwood HL, Govind CK, Wu CF (2000) Role of cAMP cascade in synaptic stability and plasticity: ultrastructural and physiological analyses of individual synaptic boutons in Drosophila memory mutants. J Neurosci 20:3980-3992.

Rheuben MB, Yoshihara M, Kidokoro Y (1999) Ultrastructural correlates of neuromuscular junction development. Int Rev Neurobiol 43:69-92.

Richmond JE, Broadie KS (2002) The synaptic vesicle cycle: exocytosis and endocytosis in Drosophila and C. elegans. Curr Opin Neurobiol 12:499-507.

Ritzenthaler S, Suzuki E, Chiba A (2000) Postsynaptic filopodia in muscle cells interact with innervating motoneuron axons. Nat Neurosci 3:1012-1017. 
Rosenmund C, Stern-Bach Y, Stevens CF (1998) The tetrameric structure of a glutamate receptor channel. Science 280:1596-1599.

Rubin GM, Spradling AC (1982) Genetic transformation of Drosophila with transposable element vectors. Science 218:348-353.

Saitoe M, Schwarz TL, Umbach JA, Gundersen CB, Kidokoro Y (2002) Response: meaningless minis? Trends Neurosci 25:385-386.

Salinas PC, Price SR (2005) Cadherins and catenins in synapse development. Curr Opin Neurobiol 15:73-80.

Salvaterra PM, Kitamoto T (2001) Drosophila cholinergic neurons and processes visualized with Gal4/UAS-GFP. Brain Res Gene Expr Patterns 1:73-82.

Sambrook J, Fritsch E, Maniatis T (1989) Molecular cloning: A laboratory manual, 2nd Edition. Cold Spring Harbor, NY: Cold Spring Harbor Laboratory Press.

Sanes JR, Lichtman JW (1999) Development of the vertebrate neuromuscular junction. Annu Rev Neurosci 22:389-442.

Sans N, Racca C, Petralia RS, Wang YX, McCallum J, Wenthold RJ (2001) Synapse-associated protein 97 selectively associates with a subset of AMPA receptors early in their biosynthetic pathway. J Neurosci 21:7506-7516.

Schuster CM, Davis GW, Fetter RD, Goodman CS (1996) Genetic dissection of structural and functional components of synaptic plasticity. II. Fasciclin II controls presynaptic structural plasticity. Neuron 17:655-667.

Schuster CM, Davis GW, Fetter RD, Goodman CS (1996) Genetic dissection of structural and functional components of synaptic plasticity. I. Fasciclin II controls synaptic stabilization and growth. Neuron 17:641-654.

Schuster CM, Davis GW, Fetter RD, Goodman CS (1996) Genetic dissection of structural and functional components of synaptic plasticity. I. Fasciclin II controls synaptic stabilization and growth. Neuron 17:641-654.

Schuster CM, Ultsch A, Schloss P, Cox JA, Schmitt B, Betz H (1991) Molecular cloning of an invertebrate glutamate receptor subunit expressed in Drosophila muscle. Science 254:112-114.

Shapira M, Zhai RG, Dresbach T, Bresler T, Torres VI, Gundelfinger ED, Ziv NE, Garner CC (2003) Unitary assembly of presynaptic active zones from PiccoloBassoon transport vesicles. Neuron 38:237-252.

Shayan AJ, Atwood HL (2000) Synaptic ultrastructure in nerve terminals of Drosophila larvae overexpressing the learning gene dunce. J Neurobiol 43:8997.

Sheng M, Kim MJ (2002) Postsynaptic signaling and plasticity mechanisms. Science 298:776-780.

Sheridan DL, Berlot CH, Robert A, Inglis FM, Jakobsdottir KB, Howe JR, Hughes TE (2002) A new way to rapidly create functional, fluorescent fusion proteins: random insertion of GFP with an in vitro transposition reaction. BMC Neurosci 3:7.

Shi S, Hayashi Y, Esteban JA, Malinow R (2001) Subunit-specific rules governing AMPA receptor trafficking to synapses in hippocampal pyramidal neurons. Cell 105:331-343.

Shi SH (2001) Amersham Biosciences \& Science Prize. AMPA receptor dynamics and synaptic plasticity. Science 294:1851-1852.

Shi SH, Hayashi Y, Petralia RS, Zaman SH, Wenthold RJ, Svoboda K, Malinow R (1999) Rapid spine delivery and redistribution of AMPA receptors after synaptic NMDA receptor activation. Science 284:1811-1816.

Sigrist SJ, Thiel PR, Reiff DF, Schuster CM (2002) The postsynaptic glutamate receptor subunit DGluR-IIA mediates long-term plasticity in Drosophila. J Neurosci 22:7362-7372. 
Sigrist SJ, Reiff DF, Thiel PR, Steinert JR, Schuster CM (2003) Experiencedependent strengthening of Drosophila neuromuscular junctions. J Neurosci 23:6546-6556.

Sigrist SJ, Thiel PR, Reiff DF, Lachance PE, Lasko P, Schuster CM (2000) Postsynaptic translation affects the efficacy and morphology of neuromuscular junctions. Nature 405:1062-1065.

Sone M, Suzuki E, Hoshino M, Hou D, Kuromi H, Fukata M, Kuroda S, Kaibuchi K, Nabeshima Y, Hama C (2000) Synaptic development is controlled in the periactive zones of Drosophila synapses. Development 127:4157-4168.

Song I, Huganir RL (2002) Regulation of AMPA receptors during synaptic plasticity. Trends Neurosci 25:578-588.

Steinert JR, Kuromi H, Hellwig A, Knirr M, Wyatt AW, Kidokoro Y, Schuster CM (2006) Experience-dependent formation and recruitment of large vesicles from reserve pool. Neuron 50:723-733.

Stewart BA, Atwood HL, Renger JJ, Wang J, Wu CF (1994) Improved stability of Drosophila larval neuromuscular preparations in haemolymph-like physiological solutions. J Comp Physiol [A] 175:179-191.

Sweeney ST, Broadie K, Keane J, Niemann H, O'Kane CJ (1995) Targeted expression of tetanus toxin light chain in Drosophila specifically eliminates synaptic transmission and causes behavioral defects. Neuron 14:341-351.

Tada T, Sheng M (2006) Molecular mechanisms of dendritic spine morphogenesis. Curr Opin Neurobiol 16:95-101.

Thomas P, Mortensen M, Hosie AM, Smart TG (2005) Dynamic mobility of functional GABAA receptors at inhibitory synapses. Nat Neurosci 8:889-897.

Thomas U, Kim E, Kuhlendahl S, Koh YH, Gundelfinger ED, Sheng M, Garner CC, Budnik V (1997) Synaptic clustering of the cell adhesion molecule fasciclin II by discs- large and its role in the regulation of presynaptic structure. Neuron 19:787-799.

Thomas U, Kim E, Kuhlendahl S, Koh YH, Gundelfinger ED, Sheng M, Garner CC, Budnik V (1997) Synaptic clustering of the cell adhesion molecule fasciclin II by discs-large and its role in the regulation of presynaptic structure. Neuron 19:787-799.

Tomita S, Adesnik H, Sekiguchi M, Zhang W, Wada K, Howe JR, Nicoll RA, Bredt DS (2005) Stargazin modulates AMPA receptor gating and trafficking by distinct domains. Nature 435:1052-1058.

Varoqueaux F, Sigler A, Rhee JS, Brose N, Enk C, Reim K, Rosenmund C (2002) Total arrest of spontaneous and evoked synaptic transmission but normal synaptogenesis in the absence of Munc13-mediated vesicle priming. Proc Natl Acad Sci U S A 99:9037-9042.

Verhage M, Maia AS, Plomp JJ, Brussaard AB, Heeroma JH, Vermeer $\mathrm{H}$, Toonen RF, Hammer RE, van den Berg TK, Missler M, Geuze HJ, Sudhof TC (2000) Synaptic assembly of the brain in the absence of neurotransmitter secretion. Science 287:864-869.

Verstreken P, Bellen HJ (2002) Meaningless minis? Mechanisms of neurotransmitter-receptor clustering. Trends Neurosci 25:383-385.

Wagh DA, Rasse TM, Asan E, Hofbauer A, Schwenkert I, Durrbeck H, Buchner S, Dabauvalle MC, Schmidt M, Qin G, Wichmann C, Kittel R, Sigrist SJ, Buchner E (2006) Bruchpilot, a protein with homology to ELKS/CAST, is required for structural integrity and function of synaptic active zones in Drosophila. Neuron 49:833-844.

Walikonis RS, Jensen ON, Mann M, Provance DW, Jr., Mercer JA, Kennedy MB (2000) Identification of proteins in the postsynaptic density fraction by mass spectrometry. J Neurosci 20:4069-4080. 
Washbourne P, Bennett JE, McAllister AK (2002) Rapid recruitment of NMDA receptor transport packets to nascent synapses. Nat Neurosci 5:751-759.

Wenthold RJ, Petralia RS, Blahos J, II, Niedzielski AS (1996) Evidence for multiple AMPA receptor complexes in hippocampal CA1/CA2 neurons. J Neurosci 16:1982-1989.

Whiteheart SW, Rossnagel K, Buhrow SA, Brunner M, Jaenicke R, Rothman JE (1994) N-ethylmaleimide-sensitive fusion protein: a trimeric ATPase whose hydrolysis of ATP is required for membrane fusion. J Cell Biol 126:945-954.

Wilson MH, Deschenes MR (2005) The neuromuscular junction: anatomical features and adaptations to various forms of increased, or decreased neuromuscular activity. Int J Neurosci 115:803-828.

Wollmuth LP, Sobolevsky Al (2004) Structure and gating of the glutamate receptor ion channel. Trends Neurosci 27:321-328.

Wucherpfennig T, Wilsch-Brauninger M, Gonzalez-Gaitan M (2003) Role of Drosophila Rab5 during endosomal trafficking at the synapse and evoked neurotransmitter release. J Cell Biol 161:609-624.

Xia J, Zhang X, Staudinger J, Huganir RL (1999) Clustering of AMPA receptors by the synaptic PDZ domain-containing protein PICK1. Neuron 22:179-187.

Yoshihara M, Adolfsen B, Galle KT, Littleton JT (2005) Retrograde signaling by Syt 4 induces presynaptic release and synapse-specific growth. Science 310:858863.

Yuste R, Bonhoeffer T (2001) Morphological changes in dendritic spines associated with long-term synaptic plasticity. Annu Rev Neurosci 24:1071-1089.

Zhai RG, Bellen HJ (2004) The architecture of the active zone in the presynaptic nerve terminal. Physiology (Bethesda) 19:262-270.

Zhong Y, Wu CF (2004) Neuronal activity and adenylyl cyclase in environmentdependent plasticity of axonal outgrowth in Drosophila. J Neurosci 24:14391445.

Zhong Y, Budnik V, Wu CF (1992) Synaptic plasticity in Drosophila memory and hyperexcitable mutants: role of cAMP cascade. J Neurosci 12:644-651.

Zito K, Fetter RD, Goodman CS, Isacoff EY (1997) Synaptic clustering of Fascilin II and Shaker: essential targeting sequences and role of Dlg. Neuron 19:10071016.

Zito K, Fetter RD, Goodman CS, Isacoff EY (1997) Synaptic clustering of Fascilin II and Shaker: essential targeting sequences and role of Dlg. Neuron 19:10071016.

Ziv NE, Garner CC (2004) Cellular and molecular mechanisms of presynaptic assembly. Nat Rev Neurosci 5:385-399.

\subsection{World wide web}

A flymove.uni-muenster.de-Genetics-Flies-LifeCycle-LifeCyclePict-life_cycle.jpg (07/11/2006)

B http://rsb.info.nih.gov/ij/download.html (07/11/2006)

C http://synapses.mcg.edu/anatomy/dendrite/dendrite.stm (07/18/2006) 


\section{Appendix}

\subsection{Vectors}

pSL1180:

(Amersham Pharmacia Biotech, Buckinghamshire, England)

Fig. 56. pSL1180

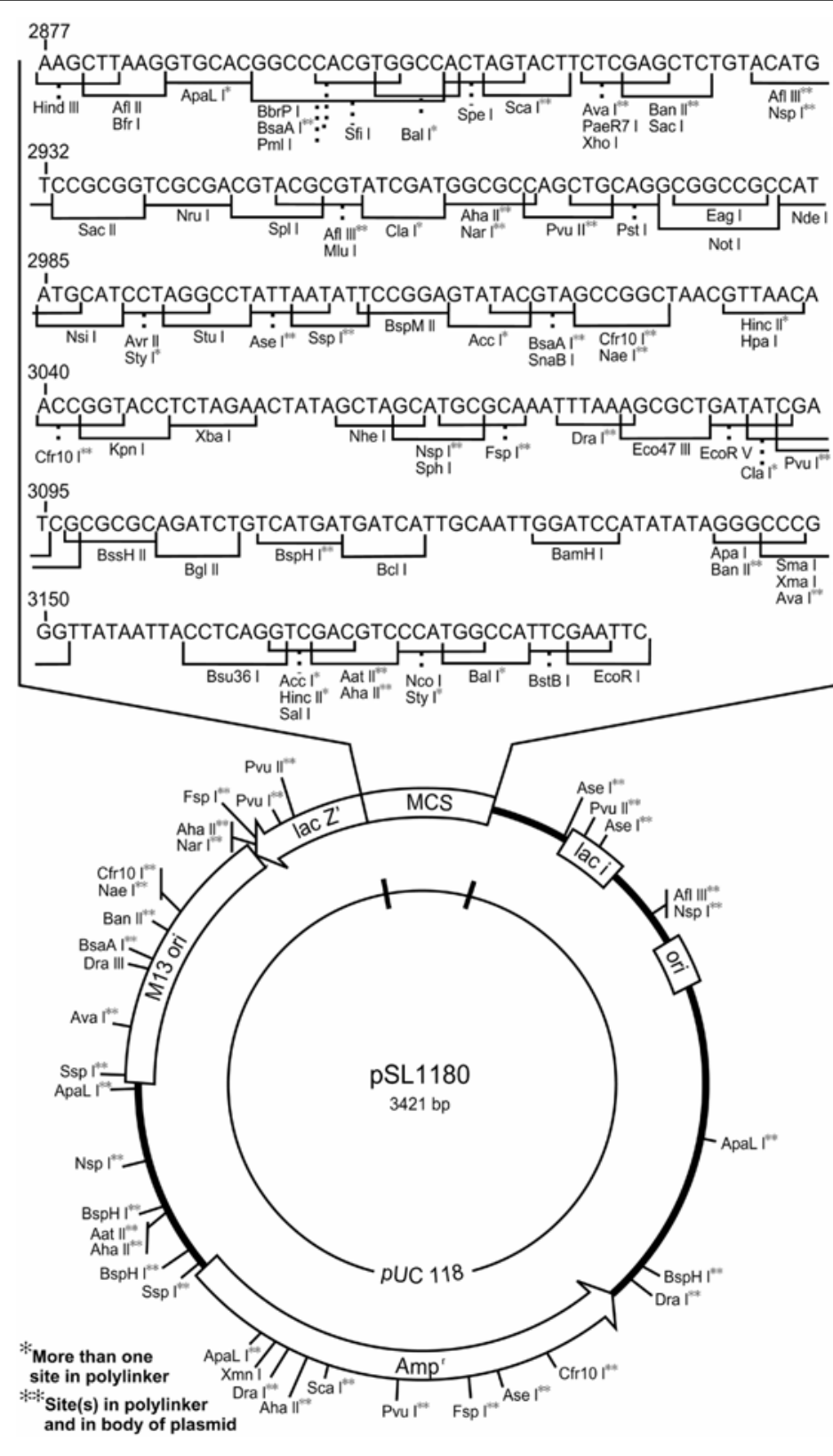




\section{pUAST:}

Fig. 57. pUAST Adapted from (Brand and Perrimon, 1993)
5. BamHI HindIII PetI 8700

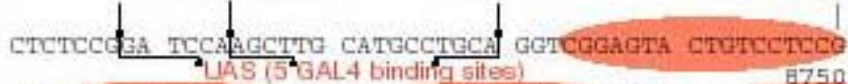
AGCGGAGTAC TUTCCTCCOA GCGGAGTACT GTECTCCGAG CGGAGTACTG

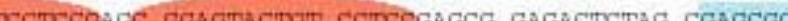
VEP70 TATA 8850 AGTATAAATA GAGGCGCITL GTCTACGGAC CGACAATTCA ATTLAAACAA B900 GCAAAGIGAA CACGTCGCTA AGCGAAAGCT AAGCAAATAA ACAAGCGCAG

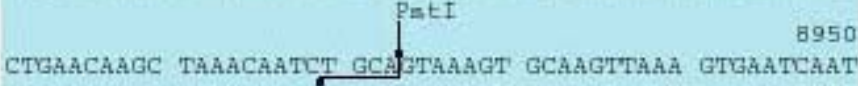
9000

taakagtaac cagcaaccaa gTaAatcaac tgGaActact gaAatctocC BCORI $9050 / 10$ AAGAAGTAAT TATTGAATAC AAGAAGAGAA CTCTGAATAG GGAATTGGQA

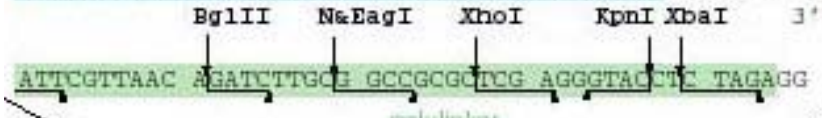

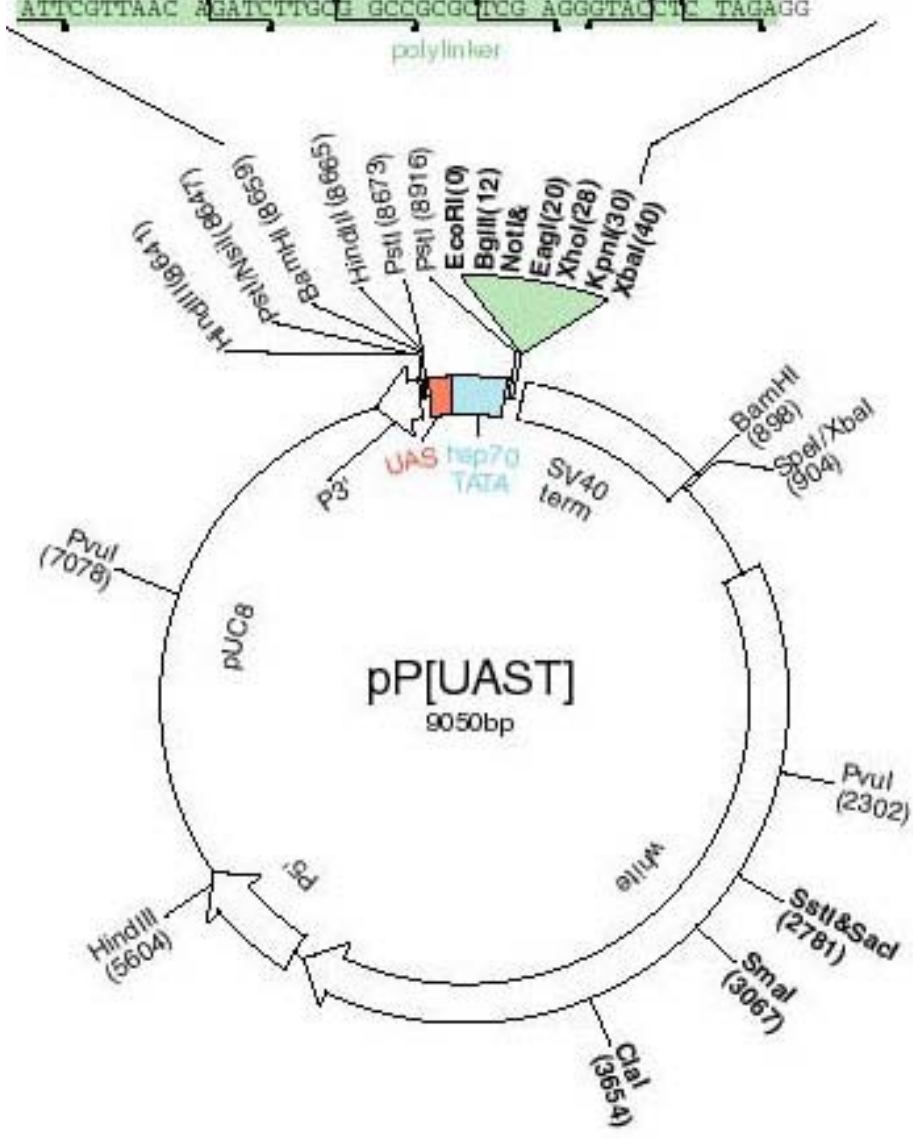


pUAST XL+:

pUAST XL+ is a modified version of pUAST (Fig. 57).

Multiple cloning site:

5' GAATTCGTTTAAACTAGTGGCCGGCCTTAATTAAGGCGCGCCATTTAA

ATGAATTCGTTAACGATCTGCGGCCGCGGCTCGAGGGTACCTCTAGA 3'

\subsection{GluR structure and alignments}
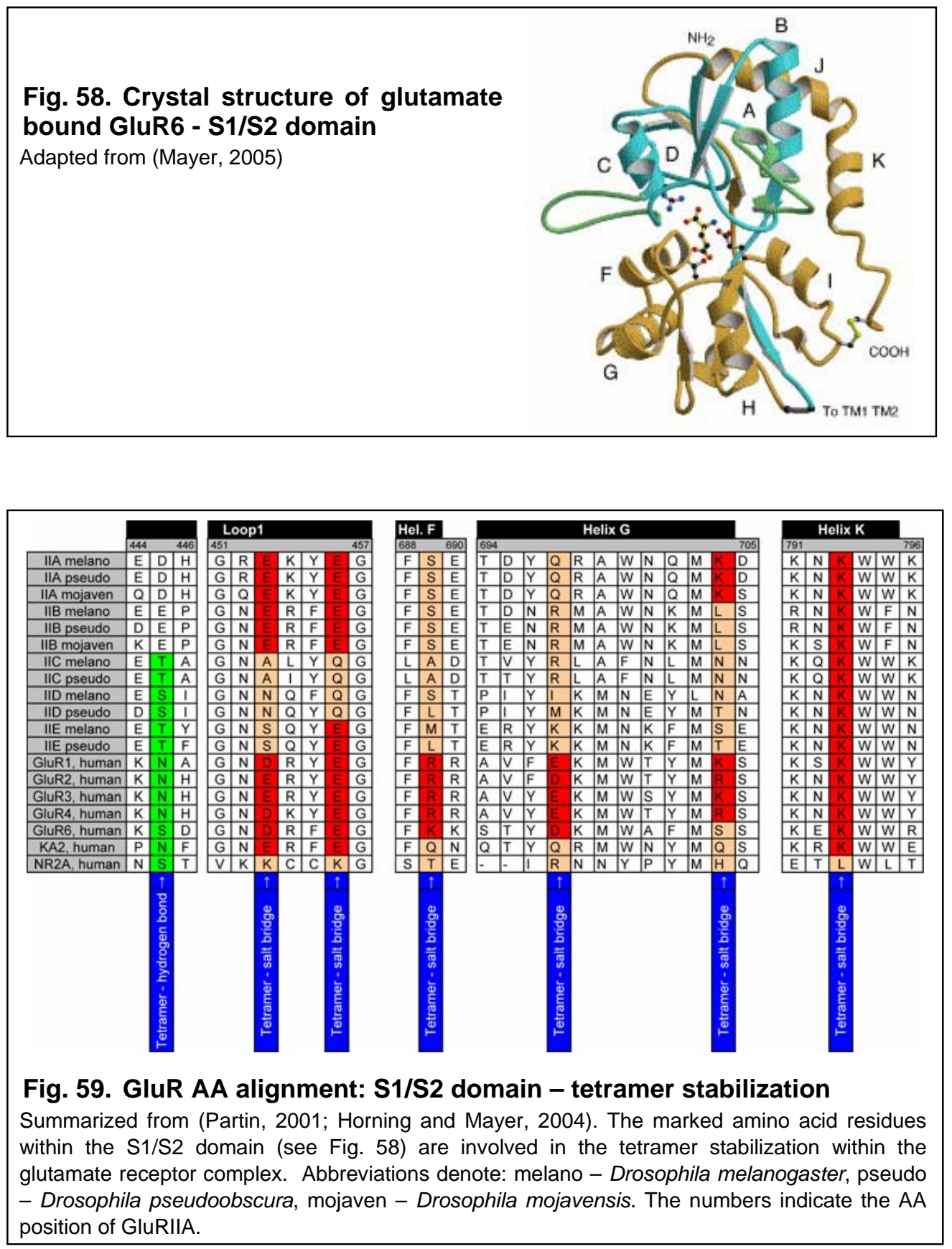

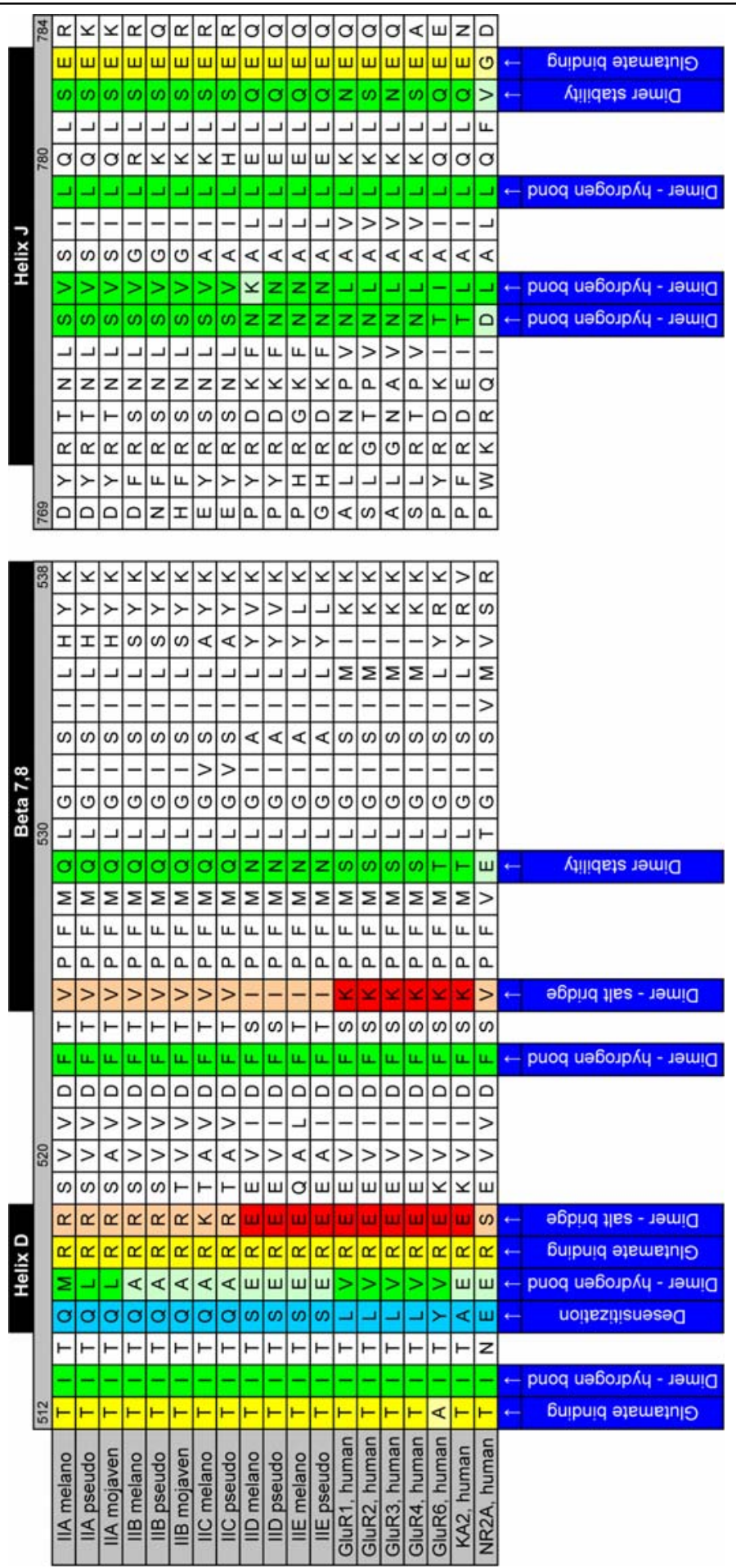

Fig. 60. GluR AA alignment: S1/S2 domain - beta 7,8, helix D and J

Summarized from (Partin, 2001; Horning and Mayer, 2004). The marked amino acid residues within the S1/S2 domain (see Fig. 58) are involved in dimer stabilization (red and green), glutamate binding (yellow) and desensitization (blue). Abbreviations denote: melano - Drosophila melanogaster, pseudo - Drosophila pseudoobscura, mojaven - Drosophila mojavensis. The numbers indicate the AA position of GluRIIA. 
Fig. 61. GluR AA alignment: reentrant pore loop and M2

Summarized from (Wollmuth and Sobolevsky, 2004). The marked amino acid residues are involved in ion gating (blue) and desensitization (red). The lurcher mutation residue (alanine to threonine) is indicated (red).

Abbreviations denote: melano Drosophila melanogaster, pseudo Drosophila pseudoobscura, mojaven Drosophila mojavensis. The numbers indicate the AA position of GluRIIA.

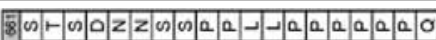

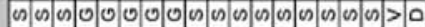
$000<\omega, 1-\omega|-1-00\rangle>>>-\omega \mid>$ $333-u \Sigma \Sigma \Sigma a a, a=\Sigma \Sigma \Sigma \Sigma \Sigma \Sigma u$

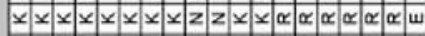

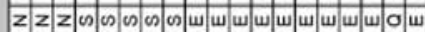

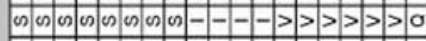

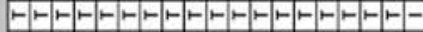
$\Delta \rightarrow---\rightarrow A-A \rightarrow-A \rightarrow-A \rightarrow=$

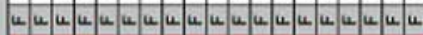

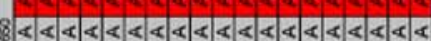

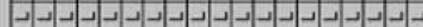
$z z z x x \cos z z z z z z z z z z z z$

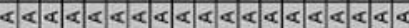

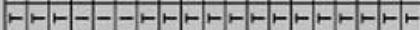

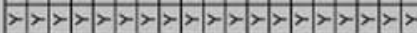

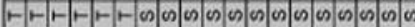

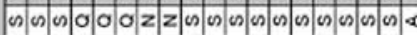

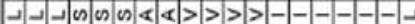
$\Sigma \equiv \equiv-----\Sigma \equiv>>-----4$

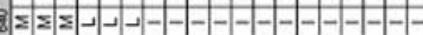

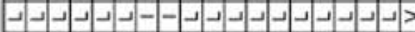

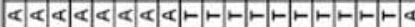

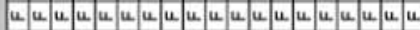

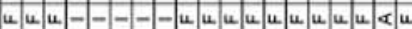
$33333333333-3333334$ 3333333333333333333 $\Sigma \Sigma \Sigma u u u \vdash t--u u>>>>->>$ $000<\&<\alpha<\&<\infty 1-0000000$

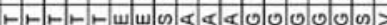
$3 \cdot \neg-\lrcorner-\neg u \cup-->>>>>>>>\sum$

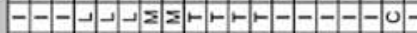

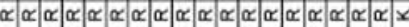
$\Sigma \Sigma \Sigma \Sigma \Sigma \Sigma>>\rightarrow r-100001 r \infty$

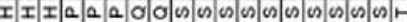

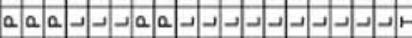
0000000 $\alpha \propto \alpha \alpha \alpha \alpha \propto \alpha x \times x \times \propto \alpha \propto \alpha x \alpha x$

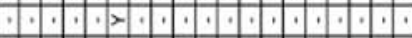

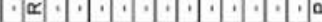

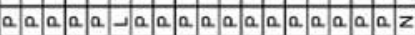

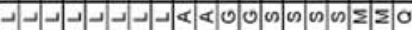
- - - $-4----------\lambda->$ $000>00 m m m \omega 0000 m m a$

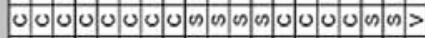
0000000000000000000

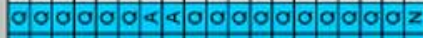

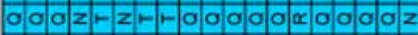

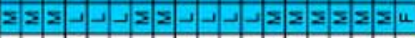

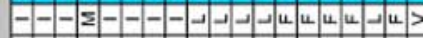

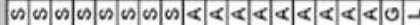
$000000<<00000000000$ $>>>-A \rightarrow>>+H-H A-A>>3$ $\Sigma \Sigma \Sigma>>>r-1-1-r \cos \cos \cup a\lrcorner$

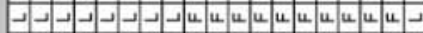
3333333333333333333

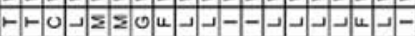

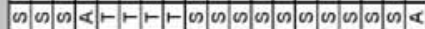
$z z z z z z z z z z z z z z z z z x$

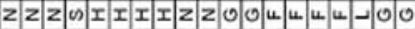
$>>>-\Sigma \Delta----H-----H-$ $z z z x z I \alpha x \mapsto \vdash \vdash$ - $000 \vdash \vdash$ $33333333 \cup u \sim u \sim u \sim u \sim>u$

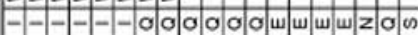

$z z z z z z z z z z z z z z z z z 0$. س山سनII

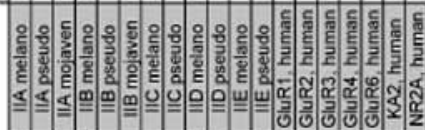




\subsection{Constructs and transgenes}

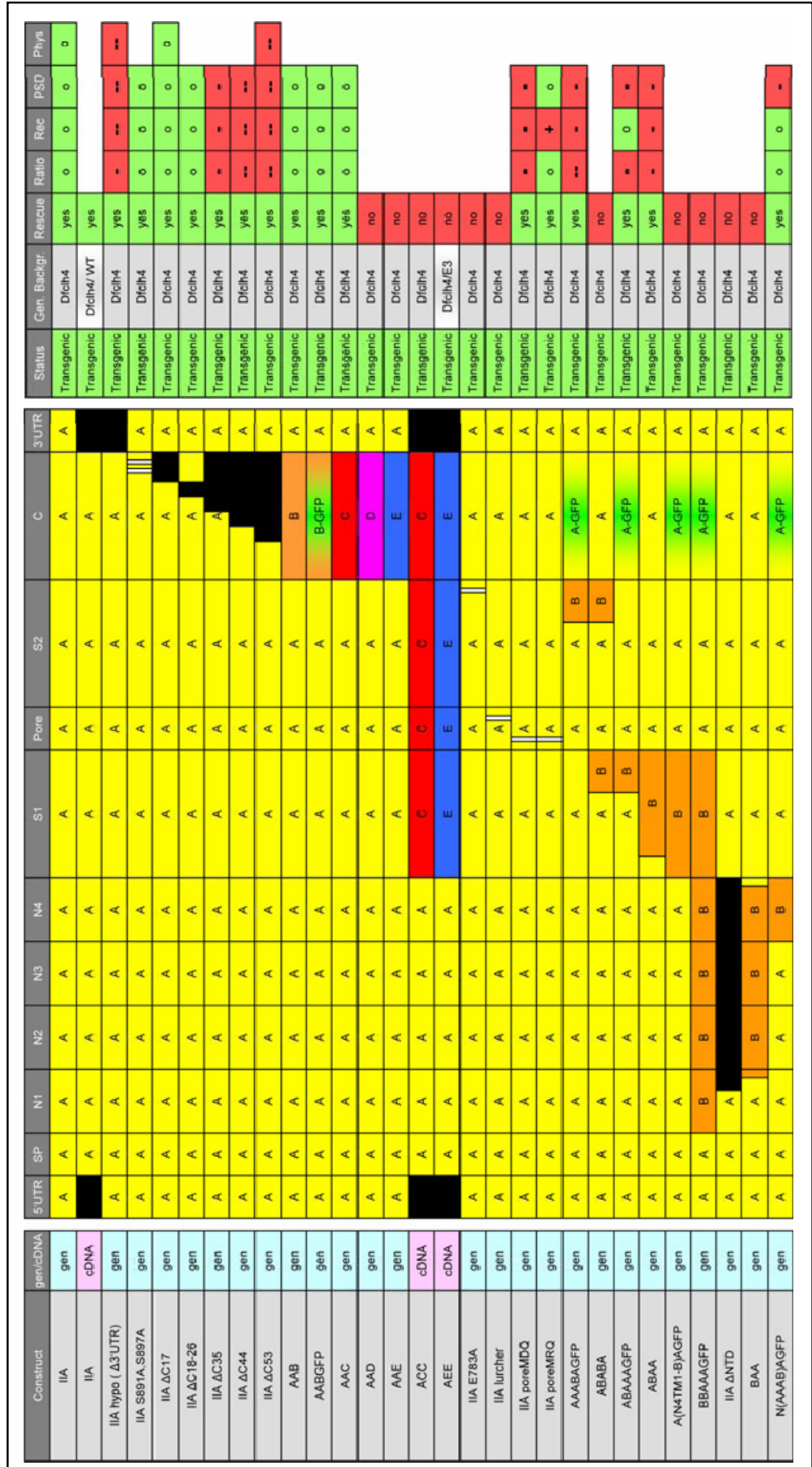

Fig. 62. Constructs and transgenes 1

Abbreviations denote: SP - signal peptide, N1-4 - NTD sub-region 1-4, C - CTD, Rec - glutamate receptor level, PSD - PSD size, Phys eEJC currents, A (yellow) - GluRIIA, B (orange) - GluRIIB, C (red) GluRIIC, D (purple) - GluRIID, E (blue) - GluRIIE, GFP - insertion of GFP, o $\rightarrow$ normal, - $\rightarrow$ reduced, $--\rightarrow$ strongly reduced, $+\rightarrow$ increased. 


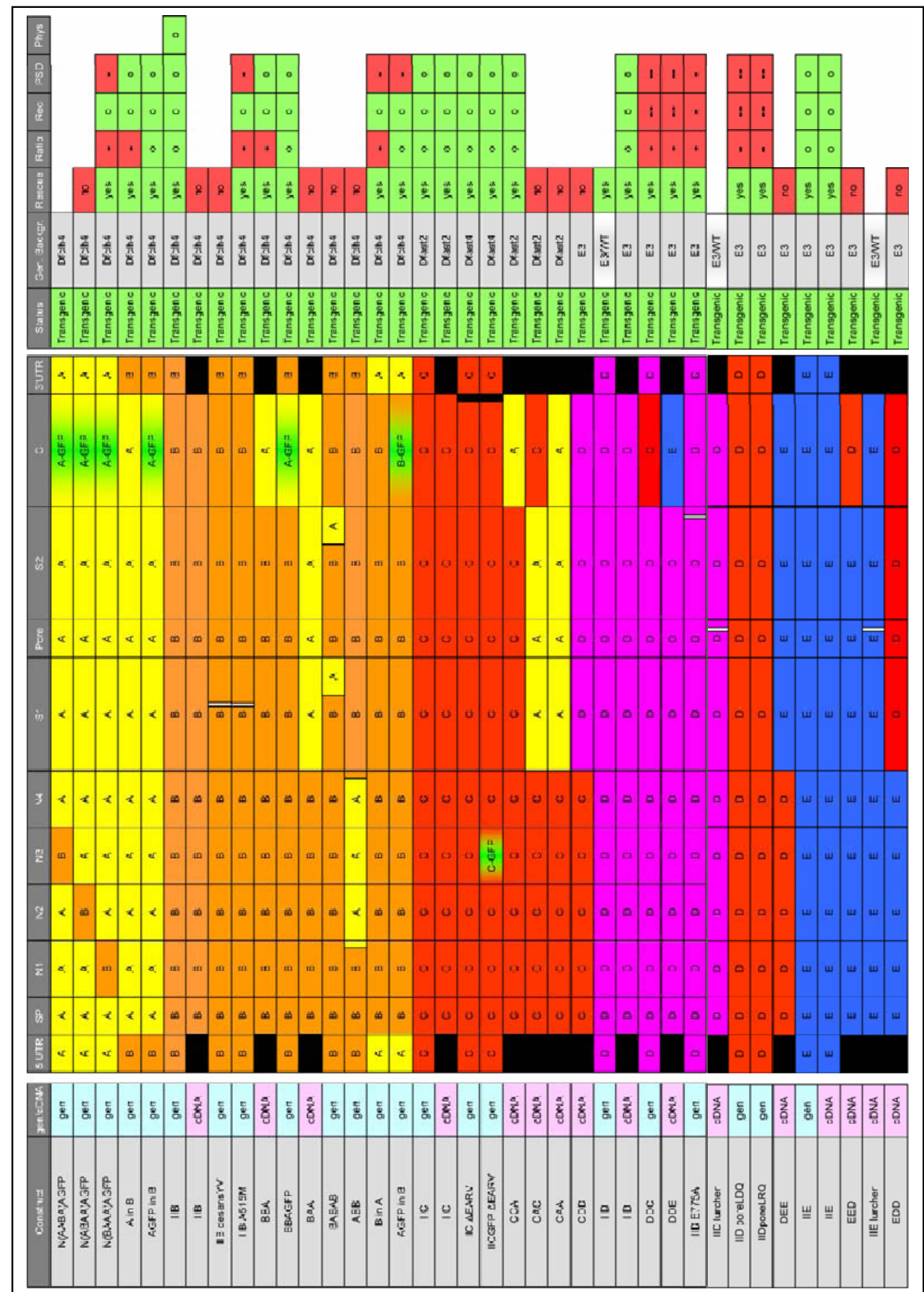

Fig. 63. Constructs and transgenes 2

Abbreviations denote: SP - signal peptide, N1-4 - NTD sub-region 1-4, C - CTD, Rec glutamate receptor level, PSD - PSD size, Phys - eEJC currents, A (yellow) - GluRIIA, B (orange) - GluRIIB, C (red) - GluRIIC, D (purple) - GluRIID, E (blue) - GluRIIE, GFP insertion of GFP, o $\rightarrow$ normal, $-\rightarrow$ reduced, $--\rightarrow$ strongly reduced. 


\begin{tabular}{|c|c|c|c|c|c|c|c|c|c|c|}
\hline Construct & Insertion sie & Location & DNA & Linker & Method & Backgr. & Status & Rescue & Signal & Comments \\
\hline \multirow[t]{15}{*}{ IIA GFP } & 28 & N1 & gen & $3 \times S G G G G$ & directed & Dfalh4 & Transgenic & no & no & \\
\hline & 52 & N1 & gen & TGPT -0 & INT & Dfclh4 & Transgenic & no & no & \\
\hline & 255 & N2 & gen & TGPT-0 & directed & Dfelh4 & Transgenic & no & no & \\
\hline & 262 & N2 & gen & TGPT-0 & INT & Dfolh4 & Transgenic & no & no & \\
\hline & 301 & N3 & CDNA & TGPT-0 & directed & Dfolh4 & Transgenic & no & no & \\
\hline & 301 & N3 & gen & TGPT-0 & directed & Dfolh4 & Transgenic & no & no & \\
\hline & 319 & N3 & CDNA & TGPT-1 & directed & Dfalh4 & Transgenic & no & no & \\
\hline & 319 & N3 & gen & TGPT-1 & directed & Dfelh4 & Transgenic & no & no & \\
\hline & 355 & N3 & gen & TGPT-0 & directed & Dfolh4 & Transgenic & no & no & \\
\hline & 427 & s1 & gen & TGPT-0 & NT & Dfelh4 & Transgenic & no & no & \\
\hline & 458 & s1 & gen & TGPT-0 & NT & Dfalh4 & Transgenic & no & no & \\
\hline & 519 & s1 & gen & TGPT-0 & INT & Dfalh4 & Transgenic & no & no & \\
\hline & 709 & s2 & gen & TGPT-0 & INT & Dfalh4 & Transgenic & no & no & \\
\hline & 760 & s2 & gen & TGPT -0 & NT & Dicih4 & Transgenic & no & no & \\
\hline & 893 & c & gen & TGPT-1 & directed & Dich4 4 & Transgenic & yes & yes & Strong signal, Phys as untagged \\
\hline HA MREP & 893 & c & gen & TGPT-1 & directed & Dfch4 4 & Transgenic & yes & yes & Strong signal \\
\hline IIA PAGFP & 893 & c & gen & TGPT-1 & directed & Dfolh 4 & Transgenic & yes & yes & Strong signal \\
\hline IIA Cameleon 3.1 & 893 & c & gen & TGPT-1 & directed & Dfcih4 4 & Transgenic & yes & yes & Strong signal \\
\hline IIA Cameleon null & 893 & c & gen & TGPT-1 & directed & Dfelh4 & Transgenic & yes & yes & Strong signal \\
\hline HA TN-XL & 893 & c & gen & TGPT-1 & directed & Dfalh4 & Transgenic & yes & yes & Very weak rescue and signal \\
\hline \multirow[t]{11}{*}{ MB GFP } & 77 & N1 & gen & TGPT-0 & NT & Dfalh4 & Transgenic & no & no & \\
\hline & 150 & N2 & gen & TGPT-0 & NT & Dich4 & Transgenic & no & no & \\
\hline & 199 & N2 & gen & TGPT-0 & INT & Dfalh4 & Transgenic & no & no & \\
\hline & 284 & N3 & gen & TGPT -0 & directed & Dfalh4 & Transgenic & no & no & \\
\hline & 287 & N3 & gen & TGPT-0 & NT & Dtelh4 & Transgenic & no & no & \\
\hline & 309 & N3 & gen & TGPT -0 & directed & Ofolh4 & Transgenic & no & no & \\
\hline & 360 & N4 & gen & TGPT-0 & INT & Ofclh4 & Transgenic & no & no & \\
\hline & 637 & Pore & gen & TGPT-0 & INT & Dfalh4 4 & Transgenic & no & no & \\
\hline & 857 & c & gen & TGPT-0 & INT & Dfalh4 & Transgenic & no & no & \\
\hline & 861 & c & gen & TGPT-0 & INT & Dfolh4 4 & Transgenic & no & no & \\
\hline & 897 & c & gen & TGPT-1 & directed & Dfelh4 & Transgenic & yes & yes & Strong signal, Phys as untagged \\
\hline MB MRFP & \$97 & c & gen & TGPT-0 & directed & Dfalh4 & Transgenic & yes & yes & Strong signal \\
\hline MB PAGFP & 897 & c & gen & TGPT-0 & directed & Dfalh4 & Transgenic & yes & yes & Rather weak signal \\
\hline \multirow[t]{4}{*}{ HC GFP } & 321 & N3 & CDNA & TGPT -0 & directed & Dfast4 & Transgenic & yes & yes & Strong signal \\
\hline & 321 & N3 & gen & TGPT -0 & directed & Dlast4 & Transgenic & no & $\infty$ & \\
\hline & 344 & N3 & CONA & TGPT-1 & directed & wT & Transgenic & & no & \\
\hline & 921 & c & CDNA & TGPT-1 & directed & Dlast 4 & Transgenic & yes & yes & Strong signal \\
\hline \multirow[t]{2}{*}{ IIC MRFP } & 321 & N3 & CDNA & TGPT -0 & directed & Dtast 4 & Transgenic & yes & yes & Strong signal \\
\hline & 921 & c & CDNA & TGPT-1 & directed & Dfast4 & Transgenic & yes & yes & Strong signal \\
\hline \multirow[t]{2}{*}{ IIC ecliptic pHlluorin } & 321 & N3 & CDNA & TGPT -0 & directed & Dfast4 & Transgenic & yes & yes & Strong signal \\
\hline & 921 & c & CDNA & TGPT-1 & directed & Dtast 4 & Transgenic & yes & yes & Strong signal \\
\hline PAK GFP & C.term & c & CDNA & none & directed & WT & Transgenic & & yes & (Too) strong signal \\
\hline PAK MREP & C.torm & c & CDNA & none & directed & WT & \begin{tabular}{|l|} 
Cloning failod \\
\end{tabular} & & & \\
\hline Synaptophysin $2 x p$ Hiluorin & internal & ? & CDNA & ? & restriction & WT & Transgenic & & yes & Specific signal \\
\hline
\end{tabular}

\section{Fig. 64. Constructs and transgenes 3}

Abbreviations denote: N1-4 - NTD sub-region 1-4, S1/2 - glutamate binding domain, C - CTD, IVT - in vitro transposition, PAGFP - photoactivatable GFP, TGPT-0/1 - transposon type (Sheridan et al., 2002), Phys - physiology, WT - wild type. For information on genetic background (Backgr.) see 3.2.2 . 


\subsection{In vivo imaging device}

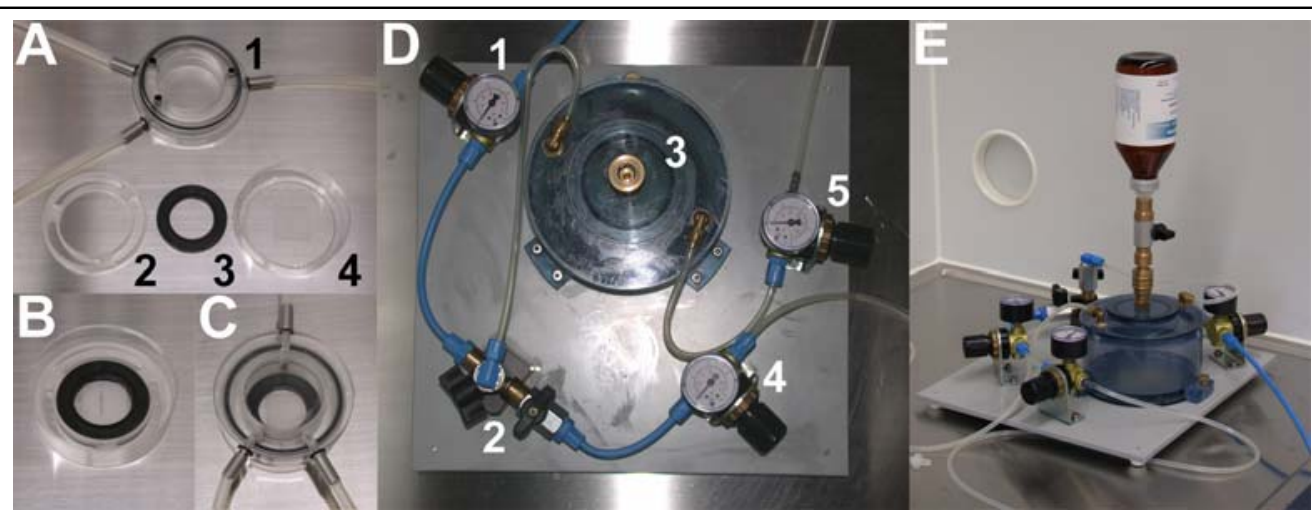

Fig. 65. In vivo imaging device

$\boldsymbol{A}$, Components of the imaging chamber: cover plate with three hose connections (1), plastic guide ring (2), iron ring (3), Petri dish with coverslip bottom and plastic disc with slit (4). $\boldsymbol{B}$, Assembled imaging chamber without the cover plate. $\boldsymbol{C}$, Completely assembled chamber. $\boldsymbol{D}$, Anesthetization device: Compressed-air supply (1), two-way valve (2), vaporization chamber (3), air supply (4), anesthetic supply (5). E, Anesthetization device with Suprane supply via an outlet adapter.

\subsection{Normalization of the PSD number and NMJ size}

In two independent experiments (wild type and IIA hypo, see Fig. 23J,K) the development of the PSD number from $2^{\text {nd }}$ to $3^{\text {rd }}$ instar larval stage was followed (NMJ 6/7, at least 6 animals per stage). On the average the PSD number (n) nearly doubled (1.92-fold). In parallel, the body wall muscles increased in size: length $(L)$, 1.82-fold, width (W), 1.95-fold, area (A), 3.59-fold. While the quotients $n / L$ and $n / W$ remained stable (1.03-fold and 0.95-fold, respectively), the quotient $\mathrm{n} / \mathrm{A}$ halved (0.51fold). Bearing in mind that the mean PSD size increased only about $0.03 \mu \mathrm{m}^{2}$ from $2^{\text {nd }}$ to $3^{\text {rd }}$ instar (see Fig. 23J,K), the average transmission strength of an individual synapse stays rather stable. Thus, normalization of the PSD number to the muscle area, which ends up in a reduction from $2^{\text {nd }}$ to $3^{\text {rd }}$ instar, is misleading as an activation of a larger muscle does not suit a reduced synapse density. At last, normalization to the segment length seems to be a more appropriate way. 


\section{Abbreviations}

- $A A$ - $\underline{a}$ mino acid

- $A B$ - antibody

- $\mathrm{ABP}$ - AMPA receptor $\underline{\text { binding protein }}$

- AChR - acetylcholine receptor

- $\mathrm{AEL}$ - after $\underline{\text { egg laying }}$

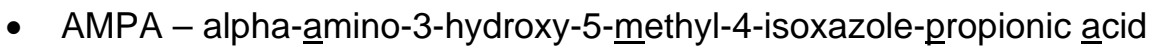

- a.u. - $\underline{\text { arbitrary } \underline{u} \text { it }}$

- $A Z$ - a ctive zone

- $\quad$ bp - $\underline{\text { base pairs }}$

- BRP - Bruchpilot protein

- cac- cacophony

- CAM - cell adhesion molecule

- CaMKII - calmodulin-dependent kinase II

- $\quad$ CAMP - cyclic adenosine monophosphate

- $\mathrm{CAZ}$ - cytomatrix at the active zone

- CNS - central nervous system

- CTD - $\underline{\text { C-terminal domain }}$

- Dlg - Discs large

- eEJC - evoked excitatory junctional current

- EGFP - enhanced green fluorescent protein

- EM - electron microscopy

- ER - endoplasmatic reticulum

- Fasll - Fasciclinll

- FRAP - fluorescence recovery after photobleaching

- $\mathrm{GABA}-\underline{y}$-äminobutyric a $\underline{\text { acid }}$

- GAL4 - galactosidase 4 protein

- GFP - green fluorescent protein

- GluR - glutamate receptor

- GRIP - glutamate receptor interacting protein

- HRP - horse radish peroxidase

- iGluR - ionotropic glutamate receptor

- $\quad$ Int $_{\text {rel }}$ - relative intensity

- IVT - in vitro transposition

- LTD - long-term depression

- LTP - long-term potentiation 
- MAGUK - membrane-âssociated quanylate kinase

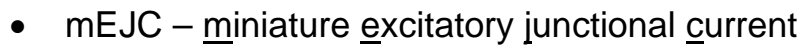

- mgv - mean gray value

- Mhc - myosin heavy chain

- $\mathrm{mRFP}$ - monomeric red fluorescent protein

- MuSK - muscle-specific kinase

- NCAM - neural cell adhesion molecule

- NGS - natural goat serum

- NSF - N-ethylmaleimide-sensitive factor

- NMDA - $\underline{\mathrm{N}}$ - $\underline{\text { methyl-}} \underline{\mathrm{D}}-\underline{a}$ spartate

- $\mathrm{NMJ}$ - neuromuscular junction

- $P A K$ - p21-âctivated kinase

- PFA - paraformaldehyde

- PICK1 - protein interacting with $\underline{\mathrm{C}}$-kinase $\underline{1}$

- PIX - PAK-interacting exchange factor

- PKA - protein kinase $\underline{A}$

- PKC - protein kinase $\underline{\mathrm{C}}$

- PSD - postsynaptic density

- $\mathrm{PSD}-95$ - postsynaptic density protein $\underline{95}$

- Ptv - presynaptic transport vesicle

- SAP97 - synapse-asssociated protein $\underline{97}$

- $\mathrm{SSR}$ - subsynaptic reticulum

- TEVC - two-electrode voltage clamp

- TNT - tetanus toxin light chain

- UAS - upstream activating sequence

- UTR - un-translated region 


\section{List of publications}

Swan LE, Wichmann C, Prange U, Schmid A, Schmidt M, Schwarz T, Ponimaskin E, Madeo F, Vorbruggen G, Sigrist SJ (2004) A glutamate receptor-interacting protein homolog organizes muscle guidance in Drosophila. Genes Dev 18:223237.

Qin G, Schwarz T, Kittel RJ, Schmid A, Rasse TM, Kappei D, Ponimaskin E, Heckmann M, Sigrist SJ (2005) Four different subunits are essential for expressing the synaptic glutamate receptor at neuromuscular junctions of Drosophila. J Neurosci 25:3209-3218.

Rasse TM, Fouquet W, Schmid A, Kittel RJ, Mertel S, Sigrist CB, Schmidt M, Guzman A, Merino C, Qin G, Quentin C, Madeo FF, Heckmann M, Sigrist SJ (2005) Glutamate receptor dynamics organizing synapse formation in vivo. Nat Neurosci 8:898-905.

Kittel RJ, Wichmann C, Rasse TM, Fouquet W, Schmidt M, Schmid A, Wagh DA, Pawlu C, Kellner RR, Willig KI, Hell SW, Buchner E, Heckmann M, Sigrist SJ (2006) Bruchpilot promotes active zone assembly, Ca2+ channel clustering, and vesicle release. Science 312:1051-1054.

Schmid A, Qin G, Wichmann C, Kittel RJ, Mertel S, Fouquet W, Schmidt M, Heckmann M, Sigrist SJ (in revision) Non-NMDA type glutamate receptors are essential for maturation but not for initial assembly of synapses at Drosophila NMJs. J Neurosci.

Schmid A, Sigrist SJ (in press) "Analysis of neuromuscular junctions: histology and in vivo imaging" in "Methods in Molecular Biology, Volume: Drosophila". Totawa, NJ: Humana Press. 


\section{Curriculum vitae}

Am 22.September 1977 wurde ich in München geboren. Ich besitze die deutsche Staatsangehörigkeit. Von 1988 bis 1997 besuchte ich das Willibald-GluckGymnasium in Neumarkt i.d.OPf., das ich 1997 mit der allgemeinen Hochschulreife abschloss. Von Oktober 1997 bis September 1998 leistete ich Ersatzdienst am Krankenhaus Rummelsberg, Schwarzenbruck. Parallel dazu absolvierte ich an der Krankenpflegehilfeschule Rummelsberg eine Ausbildung zum Krankenpflegehelfer, die ich im September 1998 abschloss. Im Oktober 1998 begann ich an der Fachhochschule Weihenstephan mein Studium zur Biotechnologie. Im März 2003 erlangte ich den Grad Dipl.-Ing.(FH). Im April 2003 begann ich in der Abteilung von Prof. Dr. Stephan Sigrist am European Neuroscience Institute in Göttingen unter der Anleitung von Prof. Dr. Ernst Wimmer die Arbeit an meiner Dissertation mit dem Titel "The role of glutamate receptors in formation and maturation of Drosophila neuromuscular synapses". 